\title{
Exceptional regions of the 2HDM parameter space
}

\author{
Howard E. Haber $\oplus^{1, *}$ and João P. Silva $\oplus^{2, \dagger}$ \\ ${ }^{1}$ Santa Cruz, Institute for Particle Physics, University of California, Santa Cruz, California 95064, USA \\ ${ }^{2}$ CFTP, Departamento de Física, Instituto Superior Técnico, Universidade de Lisboa, \\ Avenida Rovisco Pais 1, Lisboa 1049, Portugal
}

(Received 20 February 2021; accepted 13 April 2021; published 10 June 2021)

\begin{abstract}
The exceptional region of the parameter space (ERPS) of the two Higgs doublet model (2HDM) is defined to be the parameter regime where the scalar potential takes on a very special form. In the standard parametrization of the $2 \mathrm{HDM}$ scalar potential with squared mass parameters $m_{11}^{2}, m_{22}^{2}, m_{12}^{2}$, and dimensionless couplings, $\lambda_{1}, \lambda_{2}, \ldots, \lambda_{7}$, the ERPS corresponds to $\lambda_{1}=\lambda_{2}, \lambda_{7}=-\lambda_{6}, m_{11}^{2}=m_{22}^{2}$ and $m_{12}^{2}=0$, corresponding to a scalar potential with an enhanced generalized $C P$ symmetry called GCP2. Many special features persist if $\lambda_{1}=\lambda_{2}$ and $\lambda_{7}=-\lambda_{6}$ are retained while allowing for $m_{11}^{2} \neq m_{22}^{2}$ and/or $m_{12}^{2} \neq 0$, corresponding to a scalar potential with a softly broken GCP2 symmetry, which we designate as the ERPS4. In this paper, we examine many of the special features of the ERPS4, as well as even more specialized cases within the ERPS4 framework in which additional constraints on the scalar potential parameters are imposed. By surveying the landscape of the ERPS4, we complete the classification of 2HDM scalar potentials that exhibit an exact Higgs alignment (where the tree-level couplings of one neutral scalar coincide with those of the Standard Model Higgs boson), due to a residual symmetry that is unbroken in the vacuum. One surprising aspect of the ERPS4 is the possibility that the scalar sector is $C P$-conserving despite the presence of a complex parameter of the scalar potential whose complex phase cannot be removed by separate rephasings of the two scalar doublet fields. The significance of the ERPS4 regime for custodial symmetry is also discussed, and the cases where a custodial symmetric 2HDM scalar potential preserves an exact Higgs alignment are elucidated.
\end{abstract}

DOI: 10.1103/PhysRevD.103.115012

\section{INTRODUCTION}

After the discovery of the Higgs boson at the LHC [1,2], the ATLAS and CMS collaborations have ascertained that the observed properties of the Higgs boson are consistent with the corresponding predictions of the Standard Model (SM). Various production mechanisms and decay channels have been detected, and many of the observed signal strengths are consistent with SM expectations given the current precision of the LHC Higgs data, typically in the range of $10 \%-20 \%$ depending on the final state observable [3-6].

Can it be true that the scalar sector of the SM consists of a single spin-0 boson? In light of the nonminimal nature of the SM fermions (which consist of three generations of quarks and leptons) as well as the nonminimal nature of the

\footnotetext{
*haber@scipp.ucsc.edu

†jpsilva@cftp.ist.utl.pt
}

Published by the American Physical Society under the terms of the Creative Commons Attribution 4.0 International license. Further distribution of this work must maintain attribution to the author(s) and the published article's title, journal citation, and DOI. Funded by SCOAP.
SM gauge group [which is the direct product of two nonAbelian groups and the weak hypercharge $\left.\mathrm{U}(1)_{\mathrm{Y}}\right]$, it would be surprising if the scalar sector did not possess a nonminimal structure as well. Extended Higgs sectors have been proposed and explored in the literature since the birth of the Standard Model. Indeed, an important part of the LHC Higgs program is to search for the existence of new scalar states related to the observed Higgs boson, and to study their properties if found.

Of course, an arbitrary extended Higgs sector can in many cases be ruled out by current experimental data. The observed electroweak $\rho$ parameter [7-10], which is close to 1 , and the absence of tree-level Higgs-mediated flavor changing neutral currents (FCNCs) that otherwise would lead to observable FCNC effects, in conflict with current experimental bounds, impose significant constraints on any theory with an extended Higgs sector. The two Higgs doublet model (2HDM), which is one of the simplest extensions of the SM, possesses two scalar doublet fields $\Phi_{1}$ and $\Phi_{2}$ [11-13], each with the same hypercharge $Y=1$ (in a convention where the electric charge is given by $Q=T_{3}+\frac{1}{2} Y$ ). Nearly all of the new scalar physics phenomena expected in theories of extended Higgs models can be found in the $2 \mathrm{HDM}$ - charged scalars, $C P$-odd 
scalars (in models with a $C P$-conserving scalar sector) and/ or scalars of indefinite $C P$-quantum numbers (in models with a $C P$-violating scalar sector). Moreover, the $2 \mathrm{HDM}$ predicts a tree-level value of $\rho=1$ and is also compatible with the absence of tree-level Higgs-mediated FCNCs with a suitably chosen Higgs-fermion Yukawa interaction $[14,15] .{ }^{1}$ Finally, the 2HDM has often been employed in theories that introduce physics beyond the SM to solve other conceptual problems of the Standard Model. Two well-known examples are the minimal supersymmetric extension of the Standard Model (MSSM), which employs the Higgs sector of the 2HDM [19-22] and has been used to provide an explanation of the origin of the energy scale of electroweak symmetry breaking [23], and the inert doublet model (IDM) [24,25], which is a special case of the 2HDM that provides a candidate for dark matter [26-28].

Despite the simplicity of the $2 \mathrm{HDM}$, the corresponding scalar sector in its most general form is governed by 11 independent parameters [29]. However, additional theoretical assumptions can be brought to bear to reduce this large number of parameters. For example, to avoid the presence of tree-level Higgs mediated FCNCs in a natural way (i.e., without a fine-tuning of parameters in the Yukawa Lagrangian), one can introduce a $\mathbb{Z}_{2}$ discrete symmetry under which one of the Higgs doublet fields is even and the other is odd. An appropriate assignment of $\mathbb{Z}_{2}$ quantum numbers to the fermion fields then provides a symmetry explanation for the absence of Higgs-mediated tree-level FCNCs $[14,15]$. In fact, this result is robust even in the presence of a soft breaking of the $\mathbb{Z}_{2}$ symmetry by squared mass parameters appearing in the scalar potential. The softly broken $\mathbb{Z}_{2}$-symmetric $2 \mathrm{HDM}$ is governed by nine independent parameters and is called the complex 2HDM (C2HDM) [30-38].

There is some motivation to try to reduce the parameter count even further. For example, imposing $C P$ invariance on the scalar potential [11] would reduce the number of independent parameters to eight, corresponding to the vacuum expectation value, $v \simeq 246 \mathrm{GeV}$, four scalar masses, two real angles, and one scalar self-coupling. As another example, consider the requirement that one of the scalar states of the 2HDM should resemble the SM Higgs boson. One way of achieving this result is to posit an additional symmetry of the scalar potential, which would further reduce the number of independent scalar sector parameters $[39,40]$.

The exceptional region of the parameter space of the 2HDM, first introduced in Ref. [29], and designated by the acronym ERPS in Ref. [41], corresponds to a special parameter regime in which the coefficients of $\left(\Phi_{1}^{\dagger} \Phi_{1}\right)^{2}$ and $\left(\Phi_{2}^{\dagger} \Phi_{2}\right)^{2}$ appearing in the $2 \mathrm{HDM}$ scalar potential are set

\footnotetext{
${ }^{1}$ Radiative corrections to the predicted value of $\rho$ and the size of Higgs-mediated FCNCs impose some interesting constraints on the 2HDM parameter space (e.g., see Refs. [16-18]).
}

equal and the coefficient of $\left(\Phi_{1}^{\dagger} \Phi_{2}\right)\left(\Phi_{1}^{\dagger} \Phi_{1}\right)$ is the negative of the coefficient of $\left(\Phi_{1}^{\dagger} \Phi_{2}\right)\left(\Phi_{2}^{\dagger} \Phi_{2}\right)$. In addition, the squared mass coefficients of $\Phi_{1}^{\dagger} \Phi_{1}$ and $\Phi_{2}^{\dagger} \Phi_{2}$ are set equal and the squared mass coefficient of $\Phi_{1}^{\dagger} \Phi_{2}+$ H.c. is set to zero. The number of free parameters of the ERPS is five, consisting of $v$ and the four scalar masses. The ERPS conditions can be enforced by a global symmetry. Allowing for the conditions on the quadratic terms of the scalar potential to be relaxed, which would constitute a soft breaking of the global symmetry, still yields a rather exceptional region of the $2 \mathrm{HDM}$ parameter space, which we shall henceforth denote as the ERPS4 in order to emphasize that the global symmetry of the ERPS is still respected by the dimension-four terms of the scalar potential.

The ERPS4 is governed by eight parameters in its most general form, and the corresponding scalar potential is explicitly $C P$ violating. If in addition one imposes a $C P$ symmetry on the scalar potential (which may or may not be violated by the vacuum), the number of parameters is reduced by one. One can identify the seven parameters as $v$, four scalar masses, one real angle and one scalar selfcoupling. One may also impose additional softly broken symmetries within the class of the ERPS4 scalar potentials, which yields a subset of the ERPS4 with additional exceptional features. All scalar potentials obtained in this way automatically possess a $C P$-conserving scalar potential and vacuum. The 2HDM employed in the MSSM provides one such example.

It is worth highlighting a number of the exceptional features of scalar potentials that reside within the ERPS4. First, in contrast to a generic 2HDM, if the conditions on the scalar potential parameters that define the ERPS4 hold in one scalar field basis, ${ }^{2}$ then they are satisfied in all scalar field bases.

Second, in a softly broken $\mathbb{Z}_{2}$-symmetric $2 \mathrm{HDM}$, there are two potentially complex coefficients of the scalar potential, denoted by $m_{12}^{2}$ and $\lambda_{5}$ in Eq. (2.1), since the other two complex coefficients in Eq. (2.1) are $\lambda_{6}=\lambda_{7}=0$ as a consequence of the $\mathbb{Z}_{2}$ symmetry. Generically, one finds that the scalar potential is explicitly $C P$ conserving if and only if $\operatorname{Im}\left(\lambda_{5}^{*}\left[m_{12}^{2}\right]^{2}\right)=0$, since the latter condition implies that one can rephase the scalar fields $\Phi_{1}$ and $\Phi_{2}$ to remove the complex phases of $m_{12}^{2}$ and $\lambda_{5}$. The resulting

\footnotetext{
${ }^{2}$ One is always free to change the scalar field basis by redefining the scalar fields, $\Phi_{a} \rightarrow U_{a \bar{b}} \Phi_{b}$ (summed over $b=1,2$ following the index notation of Ref. [29]), where $U$ is an arbitrary $U(2)$ matrix. A particular choice for $\Phi_{1}$ and $\Phi_{2}$ is called a choice of scalar field basis. In a generic 2HDM, the squared mass coefficients and dimensionless quartic coefficients that appear in the scalar potential will be transformed by a change of scalar field basis. Often, relations among parameters that are valid in one basis cease to be valid in a different basis. The ERPS4 conditions are notable in that they hold in all scalar field bases.
} 
scalar potential is then invariant with respect to the $C P$ transformation, $\Phi \rightarrow \Phi^{*}$. Remarkably, the "only if" part of this statement is no longer true in the ERPS4. We find that in the special case of $\left\langle\Phi_{1}^{0}\right\rangle=\left\langle\Phi_{2}^{0}\right\rangle$, the scalar potential is explicitly $C P$ conserving despite the fact that $\operatorname{Im}\left(\lambda_{5}^{*}\left[m_{12}^{2}\right]^{2}\right) \neq 0$. Indeed, we can identify the modified definition of $C P$ that governs the ERPS4 in this special case. Moreover, if we constrain the ERPS4 by adding an additional softly broken global symmetry, we find that the "only if" part of the original statement is no longer true independently of the scalar field vacuum expectation values. Once again, we can understand this behavior by identifying an appropriately redefined generalized $C P$-transformation law (as shown explicitly in the Appendix C).

Third, the Higgs alignment limit [42-47] (in which the tree-level properties of one neutral scalar coincide with those of the SM Higgs boson) can be achieved by imposing a symmetry on the scalar potential $[39,40]$. For example, exact Higgs alignment is realized in the IDM, where the scalar potential and vacuum both respect a $\mathbb{Z}_{2}$ symmetry. It is of interest to classify all possible symmetries of the scalar potential beyond the $\mathbb{Z}_{2}$ symmetry of the IDM in which the Higgs alignment is exact. All scalar potentials of the ERPS fall within this class. But, one can also maintain exact Higgs alignment in some cases in which the symmetry is softly broken, corresponding to the ERPS4. Including these cases completes the classification of all symmetry based explanations for exact Higgs alignment in the 2HDM.

Fourth, it is known that custodial symmetry is an accidental symmetry of the SM Higgs potential [48,49]. In the 2HDM, the custodial symmetry is an accidental symmetry of the scalar potential if an additional constraint is imposed [50-54]. A custodial symmetric 2HDM scalar potential is automatically $C P$ conserving. Additional accidental symmetries can arise in special regions of the parameter space. Of particular interest is the case of a custodial symmetric scalar potential that preserves an exact Higgs alignment. Indeed, with two exceptions, the resulting scalar potential is necessarily in the ERPS4 regime.

In Sec. II, we introduce the $2 \mathrm{HDM}$ with a softly broken $\mathbb{Z}_{2}$-symmetric scalar potential. The possible enhanced global symmetries of the 2HDM scalar potential beyond the $\mathbb{Z}_{2}$ symmetry are summarized in Sec. III, and their connections to the ERPS are exhibited in Sec. IV. In this section, we provide a set of basis-independent conditions that correspond to the ERPS4 and special subregions of the ERPS4 where additional global symmetries (perhaps softly broken) are imposed.

A convenient scalar field basis for the ERPS4 is one where the softly broken $\mathbb{Z}_{2}$ symmetry and a softly broken permutation symmetry (that interchanges $\Phi_{1} \leftrightarrow \Phi_{2}$ ) are simultaneously imposed. We examine the properties of the resulting scalar sector in Sec. V and note that for generic choices of the parameters, the scalar potential is $C P$ violating. If the corresponding scalar potential is explicitly $C P$ conserving, then $C P$ may or may not be spontaneously broken by the vacuum. The $C P$-conserving ERPS 4 is examined in detail and we exhibit the special parameter regime where $C P$ is conserved, despite the fact that a simple rephasing of $\Phi_{1}$ and $\Phi_{2}$ is not sufficient to produce a scalar potential whose parameters are all real. In Sec. VI, we extend the softly broken $\mathbb{Z}_{2}$ symmetry to $U(1)$ and explore the properties of this special subregion of the ERPS4. One can show that the corresponding scalar potential respects a generalized $C P$ symmetry (denoted by GCP3) when expressed in a different scalar field basis. The implications of the scalar potential when expressed in terms of the GCP3 basis of scalar fields are exhibited in Sec. VII and the relations between the scalar potential parameters in the two different basis choices is made explicit in Sec. VIII.

As noted above, exact Higgs alignment is realized in the ERPS. If soft-symmetry breaking squared mass terms are included, the resulting ERPS4 may or may not exhibit exact Higgs alignment. In Sec. IX, we provide a complete classification of the symmetries (which in some cases is softly broken) that naturally yield a neutral scalar mass eigenstate whose tree-level properties are identical to those of the SM Higgs boson. In Sec. X, we combine exact Higgs alignment with the constraint of custodial symmetry and exhibit the implications for the ERPS4 regime. Conclusions and future directions appear in Sec. XI, followed by five Appendixes that provide additional details on the consequences of the ERPS4 for $C P$ symmetry and other related matters.

\section{2HDM WITH A SOFTLY BROKEN $\mathbb{Z}_{2}$-SYMMETRIC SCALAR POTENTIAL}

Let $\Phi_{1}$ and $\Phi_{2}$ denote two complex $Y=1, \mathrm{SU}(2)_{L}$ doublet scalar fields. The most general gauge invariant renormalizable scalar potential (in the $\Phi$-basis) is given by

$$
\begin{aligned}
\mathcal{V}= & m_{11}^{2} \Phi_{1}^{\dagger} \Phi_{1}+m_{22}^{2} \Phi_{2}^{\dagger} \Phi_{2}-\left[m_{12}^{2} \Phi_{1}^{\dagger} \Phi_{2}+\text { H.c. }\right] \\
& +\frac{1}{2} \lambda_{1}\left(\Phi_{1}^{\dagger} \Phi_{1}\right)^{2}+\frac{1}{2} \lambda_{2}\left(\Phi_{2}^{\dagger} \Phi_{2}\right)^{2}+\lambda_{3}\left(\Phi_{1}^{\dagger} \Phi_{1}\right)\left(\Phi_{2}^{\dagger} \Phi_{2}\right) \\
& +\lambda_{4}\left(\Phi_{1}^{\dagger} \Phi_{2}\right)\left(\Phi_{2}^{\dagger} \Phi_{1}\right)+\left\{\frac{1}{2} \lambda_{5}\left(\Phi_{1}^{\dagger} \Phi_{2}\right)^{2}\right. \\
& \left.+\left[\lambda_{6}\left(\Phi_{1}^{\dagger} \Phi_{1}\right)+\lambda_{7}\left(\Phi_{2}^{\dagger} \Phi_{2}\right)\right] \Phi_{1}^{\dagger} \Phi_{2}+\text { H.c. }\right\}
\end{aligned}
$$

In general, $m_{12}^{2}, \lambda_{5}, \lambda_{6}$ and $\lambda_{7}$ can be complex. To avoid treelevel Higgs-mediated FCNCs, we shall impose a softly broken discrete $\mathbb{Z}_{2}$ symmetry, $\Phi_{1} \rightarrow+\Phi_{1}$ and $\Phi_{2} \rightarrow-\Phi_{2}$ on the quartic terms of Eq. (2.1), which implies that $\lambda_{6}=\lambda_{7}=0$, whereas $m_{12}^{2} \neq 0$ is allowed. In this basis of scalar doublet fields (denoted as the $\mathbb{Z}_{2}$ basis), the discrete $\mathbb{Z}_{2}$ symmetry of the quartic terms of Eq. (2.1) is 
manifestly realized. In the $\mathbb{Z}_{2}$ basis, it is convenient to rephase the scalar fields such that $\lambda_{5}$ is real. Then, the requirement that $\mathcal{V}$ is bounded from below yields the following conditions $[55,56]^{3}$ :

$$
\begin{aligned}
& \lambda_{1}>0, \quad \lambda_{2}>0, \quad \lambda_{3}>-\left(\lambda_{1} \lambda_{2}\right)^{1 / 2}, \\
& \lambda_{3}+\lambda_{4} \pm \lambda_{5}>-\left(\lambda_{1} \lambda_{2}\right)^{1 / 2} .
\end{aligned}
$$

The scalar fields will develop nonzero vacuum expectation values (vevs) if the Higgs mass matrix $m_{i j}^{2}$ has at least one negative eigenvalue. We assume that the parameters of the scalar potential are chosen such that the minimum of the scalar potential respects the $\mathrm{U}(1)_{\mathrm{EM}}$ gauge symmetry. Then, the scalar field vevs are of the form

$$
\left\langle\Phi_{1}\right\rangle=\frac{v}{\sqrt{2}}\left(\begin{array}{c}
0 \\
c_{\beta}
\end{array}\right), \quad\left\langle\Phi_{2}\right\rangle=\frac{v}{\sqrt{2}}\left(\begin{array}{c}
0 \\
e^{i \xi} s_{\beta}
\end{array}\right),
$$

where $c_{\beta} \equiv \cos \beta=v_{1} / v$ and $s_{\beta} \equiv \sin \beta=v_{2} / v$ with $v \equiv\left(v_{1}^{2}+v_{2}^{2}\right)^{1 / 2} \simeq 246 \mathrm{GeV}$. By convention, $0 \leq \beta \leq \frac{1}{2} \pi$ and $0 \leq \xi<2 \pi$.

The parameters $v_{1}, v_{2}$ and $\xi$ are determined by minimizing the scalar potential. The resulting minimization conditions in the case of $\lambda_{6}=\lambda_{7}=0$ and real $\lambda_{5}$ are given by

$$
\begin{gathered}
m_{11}^{2} v_{1}=\operatorname{Re}\left(m_{12}^{2} e^{i \xi}\right) v_{2}-\frac{1}{2} \lambda_{1} v_{1}^{3}-\frac{1}{2} \lambda_{345} v_{1} v_{2}^{2} \\
m_{22}^{2} v_{2}=\operatorname{Re}\left(m_{12}^{2} e^{i \xi}\right) v_{1}-\frac{1}{2} \lambda_{2} v_{2}^{3}-\frac{1}{2} \lambda_{345} v_{2} v_{1}^{2} \\
\operatorname{Im}\left(m_{12}^{2} e^{i \xi}\right) v_{1}=\frac{1}{2} \lambda_{5} v_{1}^{2} v_{2} \sin 2 \xi \\
\operatorname{Im}\left(m_{12}^{2} e^{i \xi}\right) v_{2}=\frac{1}{2} \lambda_{5} v_{2}^{2} v_{1} \sin 2 \xi
\end{gathered}
$$

where $\lambda_{345} \equiv \lambda_{3}+\lambda_{4}+\lambda_{5} \cos 2 \xi$. Note that both Eqs. (2.6) and (2.7) are provided in case one of the vevs vanishes. If both $v_{1} \neq 0$ and $v_{2} \neq 0$, then the minimization conditions simplify,

$$
\begin{aligned}
& m_{11}^{2}=\operatorname{Re}\left(m_{12}^{2} e^{i \xi}\right) \tan \beta-\frac{1}{2} \lambda_{1} v^{2} c_{\beta}^{2}-\frac{1}{2} \lambda_{345} v^{2} s_{\beta}^{2}, \\
& m_{22}^{2}=\operatorname{Re}\left(m_{12}^{2} e^{i \xi}\right) \cot \beta-\frac{1}{2} \lambda_{2} v^{2} s_{\beta}^{2}-\frac{1}{2} \lambda_{345} v^{2} c_{\beta}^{2},
\end{aligned}
$$

${ }^{3}$ If Eq. (2.2) is satisfied then the softly broken $\mathbb{Z}_{2}$-symmetric tree-level scalar potential is said to be stable in the strong sense. If we replace $>$ with $\geq$ in one of the above inequalities (corresponding to a particular direction in field space) and then impose the condition that the sum of the quadratic terms in the same field direction is strictly positive, then the scalar potential is said to be stable in the weak sense [56].

$$
\operatorname{Im}\left(m_{12}^{2} e^{i \xi}\right)=\frac{1}{2} \lambda_{5} v^{2} s_{\beta} c_{\beta} \sin 2 \xi .
$$

The value of the potential at the minimum is given by

$$
\begin{aligned}
V_{\min }= & \frac{1}{2} v^{2}\left[m_{11}^{2} c_{\beta}^{2}+m_{22}^{2} s_{\beta}^{2}-2 \operatorname{Re}\left(m_{12}^{2} e^{i \xi}\right) s_{\beta} c_{\beta}\right. \\
& \left.+\frac{1}{4} \lambda_{1} v^{2} c_{\beta}^{4}+\frac{1}{4} \lambda_{2} v^{2} s_{\beta}^{4}+\frac{1}{2} \lambda_{345} v^{2} s_{\beta}^{2} c_{\beta}^{2}\right] \\
= & -\frac{1}{8} v^{4}\left[\lambda_{1} c_{\beta}^{4}+\lambda_{2} s_{\beta}^{4}+2 \lambda_{345} s_{\beta}^{2} c_{\beta}^{2}\right]
\end{aligned}
$$

after making use of Eqs. (2.8) and (2.9). In light of Eq. (2.2), $V_{\min }<0$, which means that the extremum with $v_{1}=v_{2}=0$ always is less favorable than the asymmetric minimum, assuming that there is a solution to Eqs. (2.4)-(2.7) with nonvanishing vevs.

If one of the two vevs vanishes, then the minimization conditions are given by

$m_{12}^{2}=0, \quad m_{22}^{2}=-\frac{1}{2} \lambda_{2} v^{2}$, if $v_{1}=0$ and $v_{2}=v$,

$m_{12}^{2}=0, \quad m_{11}^{2}=-\frac{1}{2} \lambda_{1} v^{2}$, if $v_{2}=0$ and $v_{1}=v$.

This corresponds to an inert phase in which there exists a $\mathbb{Z}_{2}$ symmetry that is respected both by the scalar potential and the vacuum. This phase exists if and only if $m_{12}^{2}=0$ and $m_{22}^{2}<0\left[m_{11}^{2}<0\right]$ in the case of $v_{1}=0\left[v_{2}=0\right]$. These two cases are physically equivalent, as they are related by a basis change where $\Phi_{1} \leftrightarrow \Phi_{2}$. The inert phase is stable if all the physical scalar squared masses are non-negative.

\section{ENHANCED GLOBAL SYMMETRIES OF THE SCALAR POTENTIAL}

The possible global symmetries of the $2 \mathrm{HDM}$ scalar potential have been classified in Refs. [41,57-59]. Starting from a generic $\Phi$-basis, these symmetries fall into two separate categories: (i) Higgs family symmetries of the form $\Phi_{a} \rightarrow U_{a b} \Phi_{b}$, and (ii) generalized $C P$ (GCP) symmetries of the form $\Phi_{a} \rightarrow U_{a b} \Phi_{b}^{*}$, where $U$ resides in a subgroup (either discrete or continuous) of U(2). Although it might appear that the number of possible symmetries is quite large, it turns out that different choices of $U$ often yield the same constraints on the 2HDM scalar potential parameters.

The full global U(2) Higgs family symmetry transformation is the largest global symmetry group under which the gauge covariant kinetic terms of the scalar fields are invariant. Moreover, the scalar potential is invariant under a global hypercharge transformation, $\mathrm{U}(1)_{\mathrm{Y}}$, which is a subgroup of $U(2)$. Thus, any enhanced Higgs family symmetries that are respected by the scalar potential would 
be a subset of the $\mathrm{U}(2)$ transformations that do not contain $\mathrm{U}(1)_{\mathrm{Y}}$ as a subgroup. We summarize below possible discrete and continuous Higgs family symmetries modulo the $\mathrm{U}(1)_{\mathrm{Y}}$ hypercharge symmetry that can impose constraints on the 2HDM scalar potential in Tables I and II.

Note that the list of symmetries in Table I contains a redundancy. Although it might appear that the $\mathbb{Z}_{2}$ and $\Pi_{2}$ discrete symmetries are distinct (as they yield different

TABLE I. Classification of the Higgs family symmetries of the scalar potential in a generic $\Phi$-basis where the symmetries are manifestly realized [41,57-59]. Note that $\mathbb{Z}_{2}$ is a subgroup of $\mathrm{U}(1)_{\mathrm{PQ}}$. The corresponding constraints on the $2 \mathrm{HDM}$ scalar potential parameters are shown in Table III.

\begin{tabular}{ll}
\hline \hline Symmetry & \multicolumn{1}{c}{ Transformation law } \\
\hline $\mathbb{Z}_{2}$ & $\Phi_{1} \rightarrow \Phi_{1}, \quad \Phi_{2} \rightarrow-\Phi_{2}$ \\
$\Pi_{2}$ (mirror symmetry) & $\Phi_{1} \leftrightarrow \Phi_{2}$ \\
$\mathrm{U}(1)_{\mathrm{PQ}}$ (Peccei-Quinn & $\Phi_{1} \rightarrow e^{-i \theta} \Phi_{1}, \quad \Phi_{2} \rightarrow e^{i \theta} \Phi_{2}$, \\
$\quad$ symmetry [60]) & for $-\frac{1}{2} \pi<\theta \leq \frac{1}{2} \pi$ \\
$\begin{array}{l}\mathrm{SO}(3) \text { (maximal Higgs } \\
\quad\end{array}$ & $\Phi_{a} \rightarrow U_{a b} \Phi_{b}$, where $U \in \mathrm{U}(2) / \mathrm{U}(1)_{\mathrm{Y}}$ \\
$\quad$ flavor symmetry) & \\
\hline \hline
\end{tabular}

TABLE II. Classification of the generalized $C P$ (GCP) symmetries of the scalar potential in the $\Phi$-basis [41,57-59]. Note that a GCP3 symmetry transformation with any value of $\theta$ that lies between 0 and $\frac{1}{2} \pi$ yields the same constrained 2HDM scalar potential. The corresponding constraints on the 2HDM scalar potential parameters are shown in Table III.

\begin{tabular}{ll}
\hline \hline Symmetry & \multicolumn{1}{c}{ Transformation law } \\
\hline GCP1 & $\Phi_{1} \rightarrow \Phi_{1}^{*}, \quad \Phi_{2} \rightarrow \Phi_{2}^{*}$ \\
GCP2 & $\Phi_{1} \rightarrow \Phi_{2}^{*}, \quad \Phi_{2} \rightarrow-\Phi_{1}^{*}$ \\
GCP3 & $\left\{\begin{array}{l}\Phi_{1} \rightarrow \Phi_{1}^{*} \cos \theta+\Phi_{2}^{*} \sin \theta \\
\Phi_{2} \rightarrow-\Phi_{1}^{*} \sin \theta+\Phi_{2}^{*} \cos \theta\end{array}\right.$, for $0<\theta<\frac{1}{2} \pi$ \\
\hline \hline
\end{tabular}

constraints on the $2 \mathrm{HDM}$ scalar potential parameters in the $\Phi$-basis), one can show that starting from a $\Pi_{2}$-symmetric scalar potential, one can find a different basis of scalar fields in which the corresponding scalar potential manifestly exhibits the $\mathbb{Z}_{2}$ symmetry, and vice versa [29]. In Table III, the constraints of the various possible Higgs family symmetries and GCP symmetries on the 2HDM scalar potential in a generic $\Phi$-basis are exhibited. In the list of symmetries, $\mathrm{U}(1)$ corresponds to $\mathrm{U}(1)_{\mathrm{PQ}}$ (henceforth, we shall suppress the PQ subscript).

One can also consider applying two of the symmetries listed above simultaneously in the same basis. It was shown in Refs. [41,57] that no new independent models arise in this way. For example, applying $\mathbb{Z}_{2}$ and $\Pi_{2}$ in the same basis yields a $\mathbb{Z}_{2} \otimes \Pi_{2}$-symmetric scalar potential that is equivalent to GCP2 when expressed in a different basis. Similarly, applying $U(1)$ and $\Pi_{2}$ in the same basis yields a $\mathrm{U}(1) \otimes \Pi_{2}$-symmetric scalar potential that is equivalent to a GCP3-symmetric scalar potential when expressed in a different basis. This equivalence of GCP3 and $U(1) \otimes \Pi_{2}$ is explicitly demonstrated in Sec. VIII.

There are a number of additional Higgs family symmetries and generalized $C P$ symmetries that are closely related to the ones displayed in Tables I and II that will be useful in our work. In Tables IV and V, we have listed three additional Higgs family symmetries and two additional GCP symmetries that can be used to constrain the parameters of the 2HDM scalar potential. The corresponding constraints are exhibited in Table VI. Given scalar potentials where $\Pi_{2}, \mathrm{U}(1)^{\prime}$ and GCP3 symmetries are manifestly realized, the basis change,

$$
\Phi_{1} \rightarrow \Phi_{1}, \quad \Phi_{2} \rightarrow i \Phi_{2}
$$

yields $m_{12}^{2} \rightarrow i m_{12}^{2}, \lambda_{5} \rightarrow-\lambda_{5}, \lambda_{6} \rightarrow i \lambda_{6}$ and $\lambda_{7} \rightarrow i \lambda_{7}$, and produces a scalar potential where $\Pi_{2}^{\prime}, \mathrm{U}(1)^{\prime \prime}$ and GCP3' symmetries, respectively, are manifestly realized.

TABLE III. Classification of 2HDM scalar potential symmetries and their impact on the parameters of the scalar potential [cf. Eq. (2.1)] in the $\Phi$-basis [41,57-59]. Empty entries correspond to a lack of constraints on the corresponding parameters. Note that $\Pi_{2}, \mathbb{Z}_{2} \otimes \Pi_{2}$ and $U(1) \otimes \Pi_{2}$ are not independent from other symmetry conditions, since a change of scalar field basis can be performed in each case to yield a new basis in which the $\mathbb{Z}_{2}$, GCP2 and GCP3 symmetries, respectively, are manifestly realized.

\begin{tabular}{|c|c|c|c|c|c|c|c|c|}
\hline Symmetry & $m_{22}^{2}$ & $m_{12}^{2}$ & $\lambda_{2}$ & $\lambda_{4}$ & $\operatorname{Re} \lambda_{5}$ & $\operatorname{Im} \lambda_{5}$ & $\lambda_{6}$ & $\lambda_{7}$ \\
\hline $\mathbb{Z}_{2}$ & & 0 & & & & & 0 & 0 \\
\hline$\Pi_{2}$ & $m_{11}^{2}$ & Real & $\lambda_{1}$ & & & 0 & & $\lambda_{6}^{*}$ \\
\hline $\mathbb{Z}_{2} \otimes \Pi_{2}$ & $m_{11}^{2}$ & 0 & $\lambda_{1}$ & & & 0 & 0 & 0 \\
\hline $\mathrm{U}(1)$ & & 0 & & & 0 & 0 & 0 & 0 \\
\hline $\mathrm{U}(1) \otimes \Pi_{2}$ & $m_{11}^{2}$ & 0 & $\lambda_{1}$ & & 0 & 0 & 0 & 0 \\
\hline $\mathrm{SO}(3)$ & $m_{11}^{2}$ & 0 & $\lambda_{1}$ & $\lambda_{1}-\lambda_{3}$ & 0 & 0 & 0 & 0 \\
\hline GCP1 & & Real & & & & 0 & Real & Real \\
\hline GCP2 & $m_{13}^{2}$ & 0 & $\lambda_{1}$ & & & & & $-\lambda_{6}$ \\
\hline GCP3 & $m_{11}^{2}$ & 0 & $\lambda_{1}$ & & $\lambda_{1}-\lambda_{3}-\lambda_{4}$ & 0 & 0 & 0 \\
\hline
\end{tabular}


TABLE IV. 2HDM scalar potential Higgs family symmetries in a generic $\Phi$-basis that are related by a simple change of basis to the family symmetries of Table I. As in the case of the Peccei-Quinn symmetry, a scalar potential that respects the $\mathrm{U}(1)$ symmetries above must be invariant for any value of $-\frac{1}{2} \pi<\theta \leq \frac{1}{2} \pi$. Note that $\Pi_{2}^{\prime}\left[\Pi_{2}\right]$ is a subgroup of $\mathrm{U}(1)^{\prime}\left[\mathrm{U}(1)^{\prime \prime}\right]$, respectively. The corresponding constraints on the $2 \mathrm{HDM}$ scalar potential parameters are shown in Table VI.

\begin{tabular}{llrr}
\hline \hline Symmetry & \multicolumn{2}{c}{ Transformation law } & Related symmetry \\
\hline$\Pi_{2}^{\prime}$ & $\Phi_{1} \rightarrow \Phi_{2}, \quad \Phi_{2} \rightarrow-\Phi_{1}$ & & $\Pi_{2}$ \\
$\mathrm{U}(1)^{\prime}$ & $\Phi_{1} \rightarrow \Phi_{1} \cos \theta+\Phi_{2} \sin \theta$, & $\Phi_{2} \rightarrow-\Phi_{1} \sin \theta+\Phi_{2} \cos \theta$ & $\mathrm{U}(1)_{\mathrm{PQ}}$ \\
$\mathrm{U}(1)^{\prime \prime}$ & $\Phi_{1} \rightarrow \Phi_{1} \cos \theta+i \Phi_{2} \sin \theta$, & $\Phi_{2} \rightarrow i \Phi_{1} \sin \theta+\Phi_{2} \cos \theta$ & $\mathrm{U}(1)_{\mathrm{PQ}}$ \\
\hline \hline
\end{tabular}

The origin of $\mathrm{U}(1)^{\prime}$ is slightly more subtle and is derived in Sec. VIII. It arises in the following way. We have noted above that the $\mathrm{U}(1) \otimes \Pi_{2}$ and GCP3 symmetries are equivalent in the sense that the scalar field bases in which these symmetries are manifestly realized are related by a change in basis. Moreover, as shown in Sec. VIII, by transforming from the $\mathrm{U}(1) \otimes \Pi_{2}$ basis to the GCP3 basis, the $\mathrm{U}(1)$ symmetry constraints are mapped onto the $\mathrm{U}(1)^{\prime}$ symmetry constraints.

Starting from a GCP1 symmetry transformation in the $\Phi$ basis, consider an arbitrary basis change, $\Phi \rightarrow \Phi^{\prime}=U \Phi$. Then, Eqs. (C18) and (C28) yield the corresponding GCP transformation in the $\Phi^{\prime}$-basis, $\Phi \rightarrow V \Phi^{*}$, where $V \equiv U U^{\top}$

TABLE V. Generalized $C P$ (GCP) symmetries of the scalar potential in the $\Phi$-basis that are related by a change of basis to the GCP symmetries of Table II. Note that a GCP3' symmetry with any value of $\theta$ that lies between 0 and $\frac{1}{2} \pi$ yields the same constrained $2 \mathrm{HDM}$ scalar potential. The corresponding constraints on the 2HDM scalar potential parameters are shown in Table VI.

\begin{tabular}{|c|c|c|}
\hline Symmetry & Transformation law & $\begin{array}{c}\text { Related } \\
\text { symmetry }\end{array}$ \\
\hline $\mathrm{GCP}^{\prime}$ & $\Phi_{1} \rightarrow \Phi_{2}^{*}, \quad \Phi_{2} \rightarrow \Phi_{1}^{*}$ & GCP1 \\
\hline $\mathrm{GCP}^{\prime}$ & $\left\{\begin{array}{l}\Phi_{1} \rightarrow \Phi_{1}^{*} \cos \theta-i \Phi_{2}^{*} \sin \theta \\
\Phi_{2} \rightarrow i \Phi_{1}^{*} \sin \theta-\Phi_{2}^{*} \cos \theta\end{array}\right.$, for $0<\theta<\frac{1}{2} \pi$ & GCP3 \\
\hline
\end{tabular}

is a symmetric unitary matrix. The choice of $V=\left(\begin{array}{ll}0 & 1 \\ 1 & 0\end{array}\right)$ corresponds to the definition of $\mathrm{GCP}^{\prime}$ exhibited in Tables V and VI. In contrast to the GCP1 symmetry, the GCP1' symmetry transformation is especially noteworthy in that it does not enforce reality conditions on the potentially complex parameters $m_{12}^{2}, \lambda_{5}, \lambda_{6}$ and $\lambda_{7}$.

Finally, it should be noted that the constraints on the scalar potential in a scalar field basis where the GCP3 symmetry is manifestly realized are precisely the same as the constraints due to the $\mathrm{U}(1)^{\prime} \otimes \mathbb{Z}_{2}$ family symmetry when imposed in the same basis of scalar fields. This should be contrasted with the $\mathrm{U}(1) \otimes \Pi_{2}$-symmetric scalar potential, which is equivalent to the GCP3-symmetric scalar potential when expressed with respect to a different scalar field basis. Likewise the parameter constraints in a basis where the GCP3' symmetry is manifestly realized coincide with those that arise from the $\mathrm{U}(1)^{\prime \prime} \otimes \mathbb{Z}_{2}$ family symmetry.

\section{AN EXCEPTIONAL REGION OF THE 2HDM PARAMETER SPACE}

The exceptional region of the parameter space (ERPS) of the $2 \mathrm{HDM}$ corresponds to a regime in which the parameters of the scalar potential satisfy the following conditions: $m_{11}^{2}=m_{22}^{2}, m_{12}^{2}=0, \lambda_{1}=\lambda_{2}$ and $\lambda_{7}=-\lambda_{6}$. These conditions can be imposed by a GCP2 symmetry,

TABLE VI. The impact of the 2HDM scalar potential symmetries listed in Tables IV and V on the parameters of the scalar potential [cf. Eq. (2.1)] in the $\Phi$-basis. Empty entries correspond to a lack of constraints on the corresponding parameters. Note that the constraints on the scalar potential parameters due to the $\mathbb{Z}_{2} \otimes \Pi_{2}$, GCP3 and GCP3' symmetries coincide with those of the $\Pi_{2} \otimes \Pi_{2}^{\prime}, \mathrm{U}(1)^{\prime} \otimes \mathbb{Z}_{2}$ and $\mathrm{U}(1)^{\prime \prime} \otimes \mathbb{Z}_{2}$ symmetries, respectively.

\begin{tabular}{|c|c|c|c|c|c|c|c|}
\hline Symmetry & $m_{22}^{2}$ & $m_{12}^{2}$ & $\lambda_{2}$ & $\operatorname{Re} \lambda_{5}$ & $\operatorname{Im} \lambda_{5}$ & $\lambda_{6}$ & $\lambda_{7}$ \\
\hline$\overline{\Pi_{2}^{\prime}}$ & $m_{11}^{2}$ & Pure imaginary & $\lambda_{1}$ & & 0 & & $-\lambda_{6}^{*}$ \\
\hline$\Pi_{2} \otimes \Pi_{2}^{\prime}$ & $m_{11}^{2}$ & 0 & $\lambda_{1}$ & & 0 & 0 & 0 \\
\hline $\mathrm{U}(1)^{\prime}$ & $m_{11}^{2}$ & Pure imaginary & $\lambda_{1}$ & $\lambda_{1}-\lambda_{3}-\lambda_{4}$ & 0 & 0 & 0 \\
\hline $\mathrm{U}(1)^{\prime \prime}$ & $m_{11}^{21}$ & Real & $\lambda_{1}$ & $\lambda_{3}+\lambda_{4}-\lambda_{1}$ & 0 & 0 & 0 \\
\hline $\mathrm{U}(1)^{\prime} \otimes \mathbb{Z}_{2}$ & $m_{11}^{2}$ & 0 & $\lambda_{1}$ & $\lambda_{1}-\lambda_{3}-\lambda_{4}$ & 0 & 0 & 0 \\
\hline $\mathrm{U}(1)^{\prime \prime} \otimes \mathbb{Z}_{2}$ & $m_{11}^{2}$ & 0 & $\lambda_{1}$ & $\lambda_{3}+\lambda_{4}-\lambda_{1}$ & 0 & 0 & 0 \\
\hline GCP1 $1^{\prime}$ & $m_{11}^{2}$ & & $\lambda_{1}$ & & & & $\lambda_{6}$ \\
\hline GCP3' & $m_{11}^{2}$ & 0 & $\lambda_{1}$ & $\lambda_{3}+\lambda_{4}-\lambda_{1}$ & 0 & 0 & 0 \\
\hline
\end{tabular}




$$
\Phi_{1} \rightarrow \Phi_{2}^{*}, \quad \Phi_{2} \rightarrow-\Phi_{1}^{*}
$$

However, in the case of a softly broken GCP2 symmetry, the conditions on the $m_{i j}^{2}$ are relaxed. In general, one can take $m_{11}^{2} \neq m_{22}^{2}$ and allow for nonzero complex values of $m_{12}^{2}$. The resulting parameter regime maintains many of the exceptional characteristics of the ERPS and will henceforth be designated as the ERPS4.

If the relations, $\lambda_{1}=\lambda_{2}$ and $\lambda_{7}=-\lambda_{6}$, hold in one scalar field basis, then they hold in all choices of the scalar field basis. Indeed, one can construct a quantity, $\mathcal{Z}$, which is explicitly given in Eq. (B5), which is manifestly basis invariant under a change of scalar field basis. Evaluating this invariant in a generic $\Phi$-basis, we obtain

$$
\mathcal{Z}=\frac{1}{4}\left(\lambda_{1}-\lambda_{2}\right)^{2}+\left|\lambda_{6}+\lambda_{7}\right|^{2}
$$

Thus, the invariant condition for the ERPS4 is $\mathcal{Z}=0$, which yields $\lambda_{1}=\lambda_{2}$ and $\lambda_{7}=-\lambda_{6}$ for any choice of the scalar field basis. Moreover as first noted in Ref. [29], there exists a choice of basis such that $\lambda_{6}=\lambda_{7}=0$ and $\lambda_{5}$ is real. This basis corresponds to an enhanced symmetry obtained by simultaneously imposing a $\mathbb{Z}_{2}$ and $\Pi_{2}$ symmetry,

$$
\begin{gathered}
\mathbb{Z}_{2}: \Phi_{1} \rightarrow \Phi_{1}, \quad \Phi_{2} \rightarrow-\Phi_{2}, \\
\Pi_{2}: \Phi_{1} \leftrightarrow \Phi_{2},
\end{gathered}
$$

on the quartic terms of the scalar potential. Indeed, this symmetry adds the constraints, $\lambda_{6}=\lambda_{7}=0$ and $\lambda_{5} \in \mathbb{R}$ to the ERPS4 conditions. That is, a softly broken GCP2symmetric scalar potential can be realized as a softly broken $\mathbb{Z}_{2} \otimes \Pi_{2}$-symmetric scalar potential in a different scalar field basis. A simple proof of this result is given below Eq. (4.20).

One can impose an additional constraint on the ERPS4 by imposing a GCP3 symmetry,

$$
\begin{aligned}
& \Phi_{1} \rightarrow \Phi_{1}^{*} \cos \theta+\Phi_{2}^{*} \sin \theta, \\
& \Phi_{2} \rightarrow-\Phi_{1}^{*} \sin \theta+\Phi_{2}^{*} \cos \theta,
\end{aligned}
$$

for all $0<\theta<\frac{1}{2} \pi$. This symmetry adds the additional constraint, $\lambda_{5}=\lambda_{1}-\lambda_{3}-\lambda_{4}$ (which implies that $\lambda_{5}$ is real). We will allow for a general soft breaking of the GCP3 symmetry so that one can again take $m_{11}^{2} \neq m_{22}^{2}$ and allow for nonzero complex values of $m_{12}^{2}$. Another possible choice for an enhanced symmetry is to impose simultaneously a $\mathrm{U}(1)$ and $\Pi_{2}$ symmetry [41],

$$
\begin{gathered}
\mathrm{U}(1): \Phi_{1} \rightarrow e^{-i \theta} \Phi_{1}, \quad \Phi_{2} \rightarrow e^{i \theta} \Phi_{2}, \\
\Pi_{2}: \Phi_{1} \leftrightarrow \Phi_{2},
\end{gathered}
$$

for any $0<\theta<\frac{1}{2} \pi$. This symmetry adds the constraint $\lambda_{5}=0$ to the $\mathbb{Z}_{2} \otimes \Pi_{2}$ symmetry. As shown explicitly in Sec. VIII, if the $2 \mathrm{HDM}$ scalar potential respects a GCP3 symmetry, then there exists a basis of scalar fields in which the symmetry can be identified as $\mathrm{U}(1) \otimes \Pi_{2}$.

A basis-invariant condition can be found that corresponds to the case in which the quartic terms of the scalar potential respect the GCP3 symmetry in some basis. The invariant was first constructed in Ref. [41] $]^{4}$ and then rederived using a different technique in Appendix B of Ref. [61]. Below, we shall review the method employed in Ref. [61] while providing additional details of the derivation.

First, we make use of the notation of Eq. (C4) to assemble the $2 \mathrm{HDM}$ scalar potential couplings into a rank four tensor denoted by $Z_{a b, c d}$. It is also convenient to introduce a related rank four tensor,

$$
\bar{Z}_{a b, c d} \equiv Z_{b a, c d}=Z_{a b, d c}
$$

where the two expressions for $\bar{Z}_{a b, c d}$ given above are equivalent in light of Eqs. (C2) and (C4). Next, we define a three-vector whose components $P_{B}$ (for $B=1,2,3$ ) are given by

$$
P_{B}=\frac{1}{4}\left(Z_{a b, c d}+\bar{Z}_{a b, c d}\right) \delta_{c a} \sigma_{d b}^{B},
$$

and a $3 \times 3$ real symmetric matrix whose elements $D_{A B}$ are given by $[61,62]^{5}$

$D_{A B}=\frac{1}{4}\left(Z_{a b, c d}+\bar{Z}_{a b, c d}\right) \sigma_{c a}^{A} \sigma_{d b}^{B}-\frac{1}{12}\left(Z_{a b, a b}+\bar{Z}_{a b, a b}\right) \delta^{A B}$,

where the $\sigma^{A}$ are the Pauli matrices and there is an implicit sum over repeated indices. Under a change of scalar field basis, $\Phi \rightarrow \Phi^{\prime}=U \Phi$, Eq. (C8) yields

$$
\begin{aligned}
P_{B} \rightarrow P_{B}^{\prime} & =\mathcal{R}_{B D} P_{D}, \\
D_{A B} \rightarrow D_{A B}^{\prime} & =\mathcal{R}_{A C} \mathcal{R}_{B D} D_{C D}=\left(\mathcal{R} D \mathcal{R}^{\top}\right)_{A B},
\end{aligned}
$$

after employing the identity $U^{\dagger} \sigma^{A} U=\mathcal{R}_{A B} \sigma^{B}$, where $\mathcal{R}$ is a real orthogonal matrix that is explicitly given by

\footnotetext{
${ }^{4}$ The published version of Ref. [41] contains some typographical errors-in Eq. (39), $\operatorname{det} \tilde{\Lambda}$ should be replaced by $-\operatorname{det} \tilde{\Lambda}$ and in Eq. (44), $\frac{1}{2}$ should be replaced by $\frac{1}{3}$. All other equations in Sec. II E of Ref. [41] are correct.

${ }^{5}$ Quantities that are invariant with respect to scalar field basis transformations can be constructed out of objects such as $D_{A B}$. Although $D_{A B}$ is not an invariant, related objects such as $\operatorname{Tr} D$, $\operatorname{det} D$ and the eigenvalues of $D$ can be used to construct invariant quantities. Ivanov published the first paper that presented this strategy in Ref. [62].
} 


$$
\mathcal{R}_{A B}=\frac{1}{2} \operatorname{Tr}\left(U^{\dagger} \sigma^{A} U \sigma^{B}\right) .
$$

Using the Kronecker product notation introduced in Eqs. (C9) and (C14), we can rewrite Eq. (4.10) in a convenient form in terms of two $4 \times 4$ matrices, $Z$ and $\bar{Z}$, where $Z$ is defined in Eq. (C5) and $\bar{Z}$ is obtained from $Z$ by interchanging $\lambda_{3} \leftrightarrow \lambda_{4}$. Then, the equivalent forms of Eqs. (4.9) and (4.10) are given by

$$
P_{B}=\frac{1}{4} \operatorname{Tr}\left[\left(\mathbb{1}_{3 \times 3} \otimes \sigma^{B}\right)(Z+\bar{Z})\right],
$$

where $\mathbb{1}_{3 \times 3}$ is the $3 \times 3$ identity matrix, and

$D_{A B}=\frac{1}{4} \operatorname{Tr}\left[\left(\sigma^{A} \otimes \sigma^{B}\right)(Z+\bar{Z})\right]-\frac{1}{12} \operatorname{Tr}(Z+\bar{Z}) \delta^{A B}$.

Using $Z_{a b, c d}=Z_{b a, d c}$ [cf. Eq. (4.8)], it follows that

$$
\operatorname{Tr}\left[\left(\sigma^{A} \otimes \sigma^{B}\right)(Z+\bar{Z})\right]=\operatorname{Tr}\left[\left(\sigma^{B} \otimes \sigma^{A}\right)(Z+\bar{Z})\right],
$$

which shows that $D$ is a symmetric matrix. Moreover,

$$
\begin{aligned}
\sum_{C} \operatorname{Tr}\left[\left(\sigma^{C} \otimes \sigma^{C}\right)(Z+\bar{Z})\right] & =\operatorname{Tr}(Z+\bar{Z}) \\
& =2\left(\lambda_{1}+\lambda_{2}+\lambda_{3}+\lambda_{4}\right),
\end{aligned}
$$

which implies that $D$ is a traceless matrix. Indeed, a straightforward calculation yields

$$
P=\left(\operatorname{Re}\left(\lambda_{6}+\lambda_{7}\right)-\operatorname{Im}\left(\lambda_{6}+\lambda_{7}\right) \frac{1}{2}\left(\lambda_{1}-\lambda_{2}\right)\right),
$$

and $^{6}$

$$
D=\left(\begin{array}{ccc}
-\frac{1}{3} \Delta+\operatorname{Re} \lambda_{5} & -\operatorname{Im} \lambda_{5} & \operatorname{Re}\left(\lambda_{6}-\lambda_{7}\right) \\
-\operatorname{Im} \lambda_{5} & -\frac{1}{3} \Delta-\operatorname{Re} \lambda_{5} & -\operatorname{Im}\left(\lambda_{6}-\lambda_{7}\right) \\
\operatorname{Re}\left(\lambda_{6}-\lambda_{7}\right) & -\operatorname{Im}\left(\lambda_{6}-\lambda_{7}\right) & \frac{2}{3} \Delta
\end{array}\right),
$$

where

$$
\Delta \equiv \frac{1}{2}\left(\lambda_{1}+\lambda_{2}\right)-\lambda_{3}-\lambda_{4}
$$

In particular, the following condition for the ERPS4, which makes use of the vector $P_{B}$ [cf. Eq. (4.17)], reproduces the invariant previously given in Eq. (4.2),

\footnotetext{
${ }^{6}$ The matrix $D$ is related to $\tilde{\Lambda}$ employed in Ref. [41] by $D=\tilde{\Lambda}-\frac{1}{3}(\operatorname{Tr} \tilde{\Lambda}) \mathbb{1}_{3 \times 3}$. Thus, if $\lambda$ is an eigenvalue of $\tilde{\Lambda}$ then $\lambda-\frac{1}{3} \operatorname{Tr} \tilde{\Lambda}$ is the corresponding eigenvalue of $D$. Consequently, the condition for degenerate eigenvalues is the same if applied to either $D$ or $\tilde{\Lambda}$. There are some advantages to employing Eq. (4.18), as the condition $\operatorname{Tr} D=0$ simplifies the algebraic manipulations.
}

$$
\mathcal{Z} \equiv \sum_{B} P_{B} P_{B}=\frac{1}{4}\left(\lambda_{1}-\lambda_{2}\right)^{2}+\left|\lambda_{6}+\lambda_{7}\right|^{2}=0
$$

We now prove that if $\lambda_{1}=\lambda_{2}$ and $\lambda_{7}=-\lambda_{6}$ in the $\Phi$-basis, then there exists a $\Phi^{\prime}$-basis, defined by $\Phi^{\prime}=U \Phi$, in which $\lambda_{1}^{\prime}=\lambda_{2}^{\prime}$ and $\operatorname{Im} \lambda_{5}^{\prime}=\lambda_{6}^{\prime}=\lambda_{7}^{\prime}=0$. In light of Eq. (4.17), if $\lambda_{1}=\lambda_{2}$ and $\lambda_{7}=-\lambda_{6}$ in the $\Phi$-basis then it follows that $P=0$. Moreover, $D$ is a real traceless symmetric matrix [cf. Eq. (4.18)], which can always be transformed into a real diagonal matrix via an orthogonal similarity transformation. Thus, there exists a real orthogonal matrix $\mathcal{R}$ [explicitly given in terms of $U$ in Eq. (4.12)] such that $P^{\prime}=\mathcal{R} P=0$ and $D^{\prime}=\mathcal{R} D \mathcal{R}^{\top}$ is diagonal. Noting the explicit forms of $P$ and $D$ given above, it follows that $\lambda_{1}^{\prime}=\lambda_{2}^{\prime}$ and $\operatorname{Im} \lambda_{5}^{\prime}=\lambda_{6}^{\prime}=\lambda_{7}^{\prime}=0$ in the $\Phi^{\prime}$-basis, as previously asserted.

Next, we demonstrate, following Ref. [61], that there exists a basis in which the Peccei-Quinn U(1) symmetry of the quartic terms of the scalar potential is manifestly realized if and only if $P_{B}$ and $D_{A B}$ can be written in the following forms:

$$
P_{B}=c_{2} q_{B}, \quad D_{A B}=c_{3}\left(q_{A} q_{B}-\frac{1}{3} \delta_{A B}\right),
$$

where the $q_{B}$ are components of a real three-vector of unit length and $c_{2}$ and $c_{3}$ are constants. It then follows that

$$
\begin{aligned}
\operatorname{Tr} D & =0, \quad \operatorname{Tr}\left(D^{2}\right)=\frac{2}{3} c_{3}^{2}, \\
\operatorname{det} D & =\frac{1}{3} \operatorname{Tr}\left(D^{3}\right)=\frac{2}{27} c_{3}^{3},
\end{aligned}
$$

which yields a characteristic equation for the eigenvalues of $D$ [cf. Eqs. (4.27)-(4.30)],

$$
x^{3}-\frac{1}{3} c_{3}^{2} x-\frac{2}{27} c_{3}^{3}=\left(x+\frac{1}{3} c_{3}\right)^{2}\left(x-\frac{2}{3} c_{3}\right)=0 .
$$

Hence, the eigenvalues of $D$ are $-\frac{1}{3} c_{3},-\frac{1}{3} c_{3}$, and $\frac{2}{3} c_{3}$. That is, if $D \neq 0$ then two of the eigenvalues of $D$ are degenerate. Moreover, in light of the eigenvalue equation,

$$
D_{A B} q_{B}=c_{3}\left(q_{A} q_{B}-\frac{1}{3} \delta_{A B}\right) q_{B}=\frac{2}{3} c_{3} q_{A},
$$

it follows that $q_{A}$ is the eigenvector of the nondegenerate eigenvalue. Thus, in a scalar field basis in which $D$ as defined by Eq. (4.18) is diagonal with two degenerate diagonal elements, it follows that $\lambda_{5}=0$ and $\lambda_{6}=\lambda_{7}$, in which case we can identify $c_{3}=\Delta$ and the unit vector $q=\left(\begin{array}{lll}0 & 1\end{array}\right)$. Applying this result for $q_{B}$ in Eq. (4.21) and comparing with Eq. (4.17) then yields $c_{2}=\frac{1}{2}\left(\lambda_{1}-\lambda_{2}\right)$ and $\lambda_{7}=-\lambda_{6}$. Hence, we conclude that $\lambda_{5}=\lambda_{6}=\lambda_{7}=0$ in the 
$D$-diagonal basis, corresponding to a softly broken $\mathrm{U}(1)_{\mathrm{PQ}^{-}}$ symmetric scalar potential.

If we now impose the ERPS4 condition on the softly broken $\mathrm{U}(1)_{\mathrm{PQ}}$-symmetric scalar potential then $\lambda_{1}=\lambda_{2}$, which implies that $P=0$. In this case, the quartic terms of the scalar potential respect a $\mathrm{U}(1) \otimes \Pi_{2}$ symmetry. Given a softly broken $\mathrm{U}(1) \otimes \Pi_{2}$-symmetric scalar potential in the $\Phi$-basis, one can perform a basis change $\Phi^{\prime}=U \Phi$ such that $^{7}$

$$
\mathcal{R}=\left(\begin{array}{ccc}
1 & 0 & 0 \\
0 & 0 & -1 \\
0 & 1 & 0
\end{array}\right)
$$

Then, Eq. (4.11) yields $P^{\prime}=0, \operatorname{Im} \lambda_{5}^{\prime}=\lambda_{6}^{\prime}=\lambda_{7}^{\prime}=0$ and

$$
-\frac{1}{3} \Delta^{\prime}+\operatorname{Re} \lambda_{5}^{\prime}=\frac{2}{3} \Delta^{\prime}=-\frac{1}{3} \Delta
$$

It follows that $\Delta^{\prime}=\operatorname{Re} \lambda_{5}^{\prime}$. That is, $\lambda_{1}^{\prime}=\lambda_{2}^{\prime}=\lambda_{3}^{\prime}+\lambda_{4}^{\prime}+\lambda_{5}^{\prime}$, and $\operatorname{Im} \lambda_{5}^{\prime}=\lambda_{6}^{\prime}=\lambda_{7}^{\prime}=0$, which are the conditions for a softly broken GCP3-symmetric scalar potential in the $\Phi^{\prime}$-basis.

Finally, if $D=P=0$ then $\lambda_{1}=\lambda_{2}=\lambda_{3}+\lambda_{4}$ and $\lambda_{5}=\lambda_{6}=\lambda_{7}=0$, corresponding to a scalar potential whose quartic terms respect an $\mathrm{SO}(3)$ symmetry. In summary, we have successfully provided simple basisinvariant conditions for the $2 \mathrm{HDM}$ with a softly broken $\mathrm{U}(1), \mathrm{GCP} 2\left[\right.$ or $\mathbb{Z}_{2} \otimes \Pi_{2}$ ], GCP3 [or $\mathrm{U}(1) \otimes \Pi_{2}$ ] and $\mathrm{SO}(3)$ symmetry, respectively.

Thus, we seek a condition that guarantees that the matrix $D$ given in Eq. (4.18) possesses two degenerate eigenvalues. In general, the characteristic equation of a generic $3 \times 3$ matrix $D$ is of the form

$$
x^{3}+a_{2} x^{2}+a_{1} x+a_{0}=0,
$$

where

$$
\begin{gathered}
a_{0}=-\operatorname{det} D=-\frac{1}{6}(\operatorname{Tr} D)^{3}+\frac{1}{2} \operatorname{Tr} D \operatorname{Tr}\left(D^{2}\right)-\frac{1}{3} \operatorname{Tr}\left(D^{3}\right), \\
a_{1}=\frac{1}{2}(\operatorname{Tr} D)^{2}-\frac{1}{2} \operatorname{Tr}\left(D^{2}\right) \\
a_{2}=-\operatorname{Tr} D
\end{gathered}
$$

The cubic equation given in Eq. (4.27) has exactly two degenerate roots if the following two conditions are satisfied $[63,64]$ :

\footnotetext{
${ }^{7}$ Using the explicit form for $U$ given by Eq. (8.1) in Eq. (4.12) yields the result exhibited in Eq. (4.25).
}

$$
\begin{gathered}
\mathcal{D} \equiv \frac{9}{2} a_{0} a_{1} a_{2}-a_{0} a_{2}^{3}+\frac{1}{4} a_{1}^{2} a_{2}^{2}-a_{1}^{3}-\frac{27}{4} a_{0}^{2}=0 \\
\text { and } a_{2}^{2} \neq 3 a_{1} .
\end{gathered}
$$

Since the matrix $D$ given in Eq. (4.18) is symmetric and traceless, the conditions that $D$ possesses exactly two degenerate eigenvalues simplify to

$$
\begin{gathered}
\mathcal{D}=-a_{1}^{3}-\frac{27}{4} a_{0}^{2}=\frac{1}{8}\left[\operatorname{Tr}\left(D^{2}\right)\right]^{3}-\frac{3}{4}\left[\operatorname{Tr}\left(D^{3}\right)\right]^{2}=0 \\
\text { and } D \neq 0 .
\end{gathered}
$$

If the quartic terms of the scalar potential exhibit a $\mathrm{U}(1) \otimes \Pi_{2}$ symmetry, then it follows that $\mathcal{Z}=\mathcal{D}=0$. Thus, we conclude that the basis-invariant condition, $\mathcal{I} \equiv \mathcal{Z}+\mathcal{D}^{2}=0$, is satisfied if and only if the quartic terms of the scalar potential exhibit a $\mathrm{U}(1) \otimes \Pi_{2}$ symmetry in some basis (which implies that the quartic terms of the scalar potential exhibits a GCP3 symmetry in some other basis). One can determine this condition explicitly by setting $\lambda \equiv \lambda_{1}=\lambda_{2}$ and $\lambda_{7}=-\lambda_{6}$ when evaluating the characteristic equation of the matrix $D$, which yields

$$
\begin{gathered}
\operatorname{Tr}\left(D^{3}\right)=\frac{2}{9} \Delta^{3}+2 \Delta\left(2\left|\lambda_{6}\right|^{2}-\left|\lambda_{5}\right|^{2}\right)+12 \operatorname{Re}\left(\lambda_{5}^{*} \lambda_{6}^{2}\right), \\
\operatorname{Tr}\left(D^{2}\right)=\frac{2}{3} \Delta^{2}+2\left|\lambda_{5}\right|^{2}+8\left|\lambda_{6}\right|^{2} .
\end{gathered}
$$

Inserting the above results into Eq. (4.32) yields an expression for $\mathcal{D}$. First, we assume that $\lambda_{6} \neq 0$, in which case the end result is

$\mathcal{D}=\left[2\left|\lambda_{6}\right|^{2}-\mathcal{R}_{56}\left(\Delta+\mathcal{R}_{56}\right)\right]^{2}\left[\left(\Delta-\mathcal{R}_{56}\right)^{2}+16\left|\lambda_{6}\right|^{2}\right]+C I_{56}^{2}$,

where $\Delta \equiv \lambda-\lambda_{3}-\lambda_{4}$,

$$
\begin{aligned}
C \equiv & \left(\Delta^{2}-\left|\lambda_{5}\right|^{2}-\mathcal{R}_{56}^{2}\right)^{2}-\mathcal{R}_{56}^{2}\left|\lambda_{5}\right|^{2} \\
& +2\left|\lambda_{6}\right|^{2}\left[\Delta^{2}+9\left(\Delta+\mathcal{R}_{56}\right)^{2}+3 \mathcal{I}_{56}^{2}+3\left|\lambda_{5}\right|^{2}+24\left|\lambda_{6}\right|^{2}\right],
\end{aligned}
$$

and

$$
\mathcal{R}_{56} \equiv \frac{\operatorname{Re}\left(\lambda_{5}^{*} \lambda_{6}^{2}\right)}{\left|\lambda_{6}\right|^{2}}, \quad \mathcal{I}_{56} \equiv \frac{\operatorname{Im}\left(\lambda_{5}^{*} \lambda_{6}^{2}\right)}{\left|\lambda_{6}\right|^{2}}
$$

Note that $\mathcal{R}_{56}^{2}+\mathcal{I}_{56}^{2}=\left|\lambda_{5}\right|^{2}$.

The product of the first two factors on the right-hand side of Eq. (4.35) is non-negative definite. Thus, one solution to the equation $\mathcal{D}=0$ can be obtained by setting

$$
\left|\lambda_{6}\right|^{2}=\frac{1}{2} \mathcal{R}_{56}\left(\Delta+\mathcal{R}_{56}\right)>0,
$$


which implies that $\Delta / \mathcal{R}_{56}>-1$ [after dividing by $\mathcal{R}_{56}^{2}$ ]. ${ }^{8}$ It then follows from Eq. (4.35) that either $\mathcal{I}_{56}=0$ or $C=0$. We now demonstrate that the latter possibility is never realized. After inserting Eq. (4.38) into the expression for $C$ given in Eq. (4.36), we obtain

$$
\begin{aligned}
C= & \mathcal{I}_{56}^{4}+\left(9 \mathcal{R}_{56}^{2}+6 \Delta \mathcal{R}_{56}-2 \Delta^{2}\right) \mathcal{I}_{56}^{2} \\
& +\left(\Delta+\mathcal{R}_{56}\right)\left(\Delta+3 \mathcal{R}_{56}\right)^{3},
\end{aligned}
$$

which is a quadratic equation whose discriminant is given by

$$
\begin{aligned}
\text { Disc } & =\left(9 \mathcal{R}_{56}^{2}+6 \Delta \mathcal{R}_{56}-2 \Delta^{2}\right)^{2}-4\left(\Delta+\mathcal{R}_{56}\right)\left(\Delta+3 \mathcal{R}_{56}\right)^{3} \\
& =-\mathcal{R}_{56}\left(4 \Delta+3 \mathcal{R}_{56}\right)^{3} .
\end{aligned}
$$

If $\Delta / \mathcal{R}_{56}>-\frac{3}{4}$ then Disc $<0$ and it follows that $C>0$ for all values of $\mathcal{I}_{56}$. Finally, if $-1<\Delta / \mathcal{R}_{56} \leq-\frac{3}{4}$, then Eq. (4.39) yields $C>0$ for any nonzero value of $\mathcal{I}_{56}$. Thus, we have shown that for $\lambda_{6} \neq 0$, if Eq. (4.38) is satisfied then $\mathcal{D}=0$ if and only if

$$
\left|\lambda_{6}\right|^{2}=\frac{1}{2} \mathcal{R}_{56}\left(\Delta+\mathcal{R}_{56}\right)>0 \quad \text { and } \quad \mathcal{I}_{56}=0 .
$$

One can rewrite the two conditions given in Eq. (4.41) as a single complex equation,

$$
\lambda_{5}^{2} \lambda_{6}^{*}+\lambda_{5} \lambda_{6}\left(\lambda_{1}-\lambda_{3}-\lambda_{4}\right)-2 \lambda_{6}^{3}=0,
$$

which must hold true for any choice of scalar field basis.

If $C>0$ were valid for all nonzero values of $\lambda_{6}$, then it would immediately follow that Eq. (4.41) is the unique solution of the equation $\mathcal{D}=0$. However one can verify that regions of the parameter space exist in which $C<0$. This seems to leave open the possibility that if $\lambda_{6} \neq 0$ then $\mathcal{D}=0$ can be satisfied with a nonzero value of $\mathcal{I}_{56}$ due to a cancellation between terms in Eq. (4.35). ${ }^{9}$

Nevertheless, we shall now argue that under the assumption that $\lambda_{6} \neq 0$, the condition $\mathcal{D}=0$ holds if and only if Eq. (4.41) is satisfied. Recall that Eq. (4.32) states that the $3 \times 3$ traceless real symmetric matrix $D$ (assumed to be nonzero) possesses a doubly degenerate eigenvalue if and only if $\mathcal{D}=0$. Moreover, any $3 \times 3$ traceless real

\footnotetext{
${ }^{8}$ Note that $\mathcal{R}_{56}$ and/or $\Delta$ can be zero. If these quantities are nonvanishing, then their individual signs can be either positive or negative.

${ }^{9}$ If such a solution existed, it would not be continuously connected to the solution given by Eq. (4.41), since any small perturbation of the scalar potential parameters from Eq. (4.41) would still yield $C>0$. We have numerically checked in Mathematica using graphical techniques that in the region of parameter space where $C<0$, there are no solutions to $\mathcal{D}=0$ for $\mathcal{I}_{56} \neq 0$ and $\lambda_{6} \neq 0$. However, it is disappointing that we are unable to analytically establish the condition $\mathcal{I}_{56}=0$ directly from $\mathcal{D}=0$ when $\lambda_{6} \neq 0$.
}

symmetric matrix that possesses a doubly degenerate eigenvalue must have the form specified in Eq. (4.21). ${ }^{10}$ We can then use the discussion below Eq. (4.24) to conclude that in the $D$-diagonal basis, $\lambda_{5}=\lambda_{6}=\lambda_{7}=0$. Performing a basis transformation to an arbitrary basis [e.g., cf. Eqs. (A9) and (A10) of Ref. [37]], it follows that $\operatorname{Im}\left(\lambda_{5}^{*} \lambda_{6}^{2}\right)=0$ in any scalar field basis. Thus, we are justified in setting $\mathcal{I}_{56}=0$ in Eq. (4.35), in which case Eqs. (4.41) and (4.42) must be valid for any choice of scalar field basis.

In the case of $\lambda_{6}=0$, one can either evaluate $\mathcal{D}$ directly using Eq. (4.32) or simply set $\left|\lambda_{6}\right|=0$ in Eq. (4.35) while keeping $\mathcal{R}_{56}$ and $\mathcal{I}_{56}$ fixed. Both procedures yield the same result:

$$
\mathcal{D}=\left|\lambda_{5}\right|^{2}\left(\Delta^{2}-\left|\lambda_{5}\right|^{2}\right)^{2} .
$$

In particular, if $\lambda_{6}=0$ then we can rephase $\Phi_{2}$ such that $\lambda_{5}$ is real, in which case either

$$
\lambda_{5}=0 \quad \text { or } \quad \lambda_{5}= \pm\left(\lambda_{1}-\lambda_{3}-\lambda_{4}\right),
$$

corresponding to the manifest realization of $U(1) \otimes \Pi_{2}$ and GCP3/GCP3', respectively, as indicated by the quartic coupling relations exhibited in Tables III and VI.

\section{THE $\mathbb{Z}_{2} \otimes \Pi_{2}$ SCALAR FIELD BASIS}

Since the softly broken GCP2-symmetric scalar potential is equivalent to a softly broken $\mathbb{Z}_{2} \otimes \Pi_{2}$-symmetric scalar potential in a different scalar field basis, we henceforth focus on the $\mathbb{Z}_{2} \otimes \Pi_{2}$ basis, where $\lambda \equiv \lambda_{1}=\lambda_{2}, \lambda_{5} \neq 0$ is real and $\lambda_{6}=\lambda_{7}=0$. The softly broken parameters, $m_{11}^{2}$, $m_{22}^{2}$ and $m_{12}^{2}$, are arbitrary with $m_{12}^{2}$ potentially complex. If we demand that the potential is bounded from below, the following conditions must be satisfied:

$\lambda>0, \quad \lambda+\lambda_{3}>0, \quad \lambda+\lambda_{3}+\lambda_{4}-\left|\lambda_{5}\right|>0$,

modulo the remarks of footnote 3 . It is convenient to introduce the parameter,

$$
R \equiv \frac{\lambda_{3}+\lambda_{4}+\lambda_{5}}{\lambda} .
$$

Using the definition of $\lambda_{345}$ given below Eq. (2.7), it follows that

$$
\lambda_{345}=\lambda R-2 \lambda_{5} \sin ^{2} \xi
$$

\footnotetext{
${ }^{10}$ Given a $3 \times 3$ traceless real symmetric matrix $D$ with eigenvalues $-c,-c$ and $2 c$ (where $c \in \mathbb{R}$ ), it then follows that there exists a real orthogonal matrix $\mathcal{R}$ such that $D=\mathcal{R} \operatorname{diag}(-c,-c, 2 c) \mathcal{R}^{\top}$. However in this case one can write $\operatorname{diag}(-c,-c, 2 c)_{A B}=$ $c_{3}\left(q_{A} q_{B}-\frac{1}{3} \delta_{A B}\right)$ with $q=(0,0,1)$ and $c_{3}=3 c$. Hence, we conclude that $D=c_{3}\left(q_{A}^{\prime} q_{B}^{\prime}-\frac{1}{3} \delta_{A B}\right)$ with unit vector $q_{A}^{\prime}=\mathcal{R}_{A B} q_{B}$.
} 


\section{A. The softly broken $\mathbb{Z}_{2} \otimes \Pi_{2}$-symmetric scalar potential with $v_{1} \neq 0$ and $v_{2} \neq 0$}

We shall first assume that $v_{1}$ and $v_{2}$ are both nonzero, or equivalently, $\sin 2 \beta \neq 0$. We then use Eqs. (2.8)-(2.10) (with $\lambda \equiv \lambda_{1}=\lambda_{2}$ ) to fix the values of $\beta$ and $\xi$. In particular,

$$
\begin{gathered}
c_{2 \beta}=\frac{m_{22}^{2}-m_{11}^{2}}{m_{11}^{2}+m_{22}^{2}+\lambda v^{2}}, \\
\cos \xi=\frac{2 \operatorname{Re} m_{12}^{2}}{s_{2 \beta}\left[m_{11}^{2}+m_{22}^{2}+\frac{1}{2} \lambda(1+R) v^{2}\right]}, \\
\sin \xi=\frac{-2 \operatorname{Im} m_{12}^{2}}{s_{2 \beta}\left[m_{11}^{2}+m_{22}^{2}+\left(\frac{1}{2} \lambda(1+R)-\lambda_{5}\right) v^{2}\right]},
\end{gathered}
$$

where $s_{2 \beta} \equiv \sin 2 \beta$ and $c_{2 \beta} \equiv \cos 2 \beta$. Writing $m_{12}^{2}=\left|m_{12}^{2}\right| e^{i \theta_{12}}$ in Eqs. (5.5) and (5.6) and imposing $\cos ^{2} \xi+\sin ^{2} \xi=1$ yields an equation that determines the phase $\theta_{12}$ in terms of $\xi$ and the other scalar potential parameters. Thus, the ERPS 4 is governed by eight real parameters: $\lambda, \lambda_{3}, \lambda_{4}, \lambda_{5},\left|m_{12}^{2}\right|, v, \beta$ and $\xi$.
It is convenient to introduce the Higgs basis as follows [29,37,65-69]. The Higgs basis fields $\mathcal{H}_{1}$ and $\mathcal{H}_{2}$ are defined by the linear combinations of $\Phi_{1}$ and $\Phi_{2}$ such that $\left\langle\mathcal{H}_{1}^{0}\right\rangle=v / \sqrt{2}$ and $\left\langle\mathcal{H}_{2}^{0}\right\rangle=0$. That is,

$$
\begin{aligned}
& \mathcal{H}_{1} \equiv c_{\beta} \Phi_{1}+s_{\beta} e^{-i \xi} \Phi_{2}, \\
& \mathcal{H}_{2}=e^{i \eta}\left[-s_{\beta} e^{i \xi} \Phi_{1}+c_{\beta} \Phi_{2}\right],
\end{aligned}
$$

where we have introduced (following Ref. [37]) the complex phase factor $e^{i \eta}$ to account for the nonuniqueness of the Higgs basis, since one is always free to rephase the Higgs basis field whose vacuum expectation value vanishes. In particular, $e^{i \eta}$ is a pseudoinvariant quantity [37] that is rephased under the unitary basis transformation, $\Phi_{a} \rightarrow U_{a \bar{b}} \Phi_{b}$, as

$$
e^{i \eta} \rightarrow(\operatorname{det} U)^{-1} e^{i \eta}
$$

where $\operatorname{det} U$ is a complex number of unit modulus. In terms of the Higgs basis fields defined in Eq. (5.7), the scalar potential is given by

$$
\begin{aligned}
\mathcal{V}= & Y_{1} \mathcal{H}_{1}^{\dagger} \mathcal{H}_{1}+Y_{2} \mathcal{H}_{2}^{\dagger} \mathcal{H}_{2}+\left[Y_{3} e^{-i \eta} \mathcal{H}_{1}^{\dagger} \mathcal{H}_{2}+\text { H.c. }\right] \\
& +\frac{1}{2} Z_{1}\left(\mathcal{H}_{1}^{\dagger} \mathcal{H}_{1}\right)^{2}+\frac{1}{2} Z_{2}\left(\mathcal{H}_{2}^{\dagger} \mathcal{H}_{2}\right)^{2}+Z_{3}\left(\mathcal{H}_{1}^{\dagger} \mathcal{H}_{1}\right)\left(\mathcal{H}_{2}^{\dagger} \mathcal{H}_{2}\right)+Z_{4}\left(\mathcal{H}_{1}^{\dagger} \mathcal{H}_{2}\right)\left(\mathcal{H}_{2}^{\dagger} \mathcal{H}_{1}\right) \\
& \left.+\left\{\frac{1}{2} Z_{5} e^{-2 i \eta}\left(\mathcal{H}_{1}^{\dagger} \mathcal{H}_{2}\right)^{2}+\left[Z_{6} e^{-i \eta}\left(\mathcal{H}_{1}^{\dagger} \mathcal{H}_{1}\right)+Z_{7} e^{-i \eta} \mathcal{H}_{2}^{\dagger} \mathcal{H}_{2}\right)\right] \mathcal{H}_{1}^{\dagger} \mathcal{H}_{2}+\text { H.c. }\right\} .
\end{aligned}
$$

The coefficients of the quadratic and quartic terms of the scalar potential in Eq. (5.9) are independent of the initial choice of the $\Phi$-basis. It then follows that $Y_{3}, Z_{5}, Z_{6}$ and $Z_{7}$ are also pseudoinvariant quantities [69] that are rephased under $\Phi_{a} \rightarrow U_{a \bar{b}} \Phi_{b}$ as

$$
\begin{aligned}
{\left[Y_{3}, Z_{6}, Z_{7}\right] } & \rightarrow(\operatorname{det} U)^{-1}\left[Y_{3}, Z_{6}, Z_{7}\right] \text { and } \\
Z_{5} & \rightarrow(\operatorname{det} U)^{-2} Z_{5} .
\end{aligned}
$$

It is straightforward to compute the corresponding Higgs basis parameters. The $Y_{i}$ are given by

$$
\begin{gathered}
Y_{1}=m_{11}^{2} c_{\beta}^{2}+m_{22}^{2} s_{\beta}^{2}-\operatorname{Re}\left(m_{12}^{2} e^{i \xi}\right) s_{2 \beta}, \\
Y_{2}=m_{11}^{2} s_{\beta}^{2}+m_{22}^{2} c_{\beta}^{2}+\operatorname{Re}\left(m_{12}^{2} e^{i \xi}\right) s_{2 \beta}, \\
Y_{3}=\left[\frac{1}{2}\left(m_{22}^{2}-m_{11}^{2}\right) s_{2 \beta}-\operatorname{Re}\left(m_{12}^{2} e^{i \xi}\right) c_{2 \beta}-i \operatorname{Im}\left(m_{12}^{2} e^{i \xi}\right)\right] e^{-i \xi}
\end{gathered}
$$

Employing Eqs. (2.8)-(2.10) (with $\lambda \equiv \lambda_{1}=\lambda_{2}$ ) to eliminate $m_{11}^{2}, m_{22}^{2}$ and $\operatorname{Im}\left(m_{12}^{2} e^{i \xi}\right)$, it follows that

$$
\begin{gathered}
Y_{2}=\frac{2 \operatorname{Re}\left(m_{12}^{2} e^{i \xi}\right)}{s_{2 \beta}}-\frac{1}{2} \lambda v^{2} \\
+\frac{1}{2} v^{2}\left[\lambda(1-R)+2 \lambda_{5} \sin ^{2} \xi\right]\left(1-\frac{1}{2} s_{2 \beta}^{2}\right) \\
Z_{1}=Z_{2}=\lambda-\frac{1}{2}\left[\lambda(1-R)+2 \lambda_{5} \sin ^{2} \xi\right] s_{2 \beta}^{2}, \\
Z_{3}=\lambda_{3}+\frac{1}{2}\left[\lambda(1-R)+2 \lambda_{5} \sin ^{2} \xi\right] s_{2 \beta}^{2} \\
Z_{4}=\lambda_{4}+\frac{1}{2}\left[\lambda(1-R)+2 \lambda_{5} \sin ^{2} \xi\right] s_{2 \beta}^{2} \\
Z_{5}=\left\{\frac{1}{2}\left[\lambda(1-R)+2 \lambda_{5} \sin ^{2} \xi\right] s_{2 \beta}^{2}\right. \\
\left.\quad+\lambda_{5}\left(\cos 2 \xi+i c_{2 \beta} \sin _{2} \xi\right)\right\} e^{-2 i \xi}, \\
=\left\{\begin{array}{l}
Z_{7} \\
\left.-\frac{1}{2}\left[\lambda(1-R)+2 \lambda_{5} \sin ^{2} \xi\right] c_{2 \beta}+\frac{1}{2} i \lambda_{5} \sin 2 \xi\right\} s_{2 \beta} e^{-i \xi}
\end{array}\right.
\end{gathered}
$$


One can also check that the scalar potential minimization conditions in the Higgs basis,

$$
Y_{1}=-\frac{1}{2} Z_{1} v^{2}, \quad Y_{3}=-\frac{1}{2} Z_{6} v^{2},
$$

are satisfied. The eight parameters that specify the ERPS4 can now be identified as $v, Y_{2}, Z_{1}, Z_{3}, Z_{4}, \operatorname{Re} Z_{5}, \operatorname{Im} Z_{5}$, and $\left|Z_{6}\right|$ after using the freedom to rephase the Higgs basis field $\mathcal{H}_{2}$ to remove the complex phase from $Z_{6}$ and $Z_{7}$ [cf. Eq. (5.10)].

The subregion of the ERPS4 where $Z_{6}=0$ is worthy of special attention. The ERPS4 condition, $Z_{6}=-Z_{7}$, along with Eq. (5.20) yields $Y_{3}=Z_{6}=Z_{7}=0$, which signals the presence of a $\mathbb{Z}_{2}$ symmetry that is manifestly realized in the Higgs basis and is unbroken by the vacuum. We recognize this scenario as a special case of the IDM, and hence we shall refer to this parameter regime as the inert limit of the softly broken $\mathbb{Z}_{2} \otimes \Pi_{2}$-symmetric scalar potential. Moreover, Higgs alignment is exact in the inert limit, as discussed in Sec. IX. The conditions for achieving the inert limit will be elucidated below Eq. (5.59).
Three additional limiting cases are noteworthy. First, if $\lambda_{5}=0$, then the quartic terms of the scalar potential exhibit a $U(1) \otimes \Pi_{2}$ symmetry, which will be discussed in more detail in Sec. VI. Second, if $R=1$, then the quartic terms of the scalar potential exhibit a GCP3 symmetry, which will be discussed in more detail in Sec. VII. Both of these limits yield the same physical scalar sector, since they correspond to the softly broken GCP3-symmetric scalar potential expressed in two different choices of the scalar field basis. Finally, if $\lambda_{5}=0$ and $R=1$, then the quartic terms of the scalar potential exhibit an $\mathrm{SO}(3)$ symmetry.

The charged Higgs mass is given by

$$
\begin{aligned}
m_{H^{ \pm}}^{2} & =Y_{2}+\frac{1}{2} Z_{3} v^{2} \\
& =\frac{2 \operatorname{Re}\left(m_{12}^{2} e^{i \xi}\right)}{s_{2 \beta}}-\frac{1}{2} v^{2}\left(\lambda_{4}+\lambda_{5} \cos 2 \xi\right) .
\end{aligned}
$$

The squared masses of the neutral Higgs bosons are given by the eigenvalues of the neutral scalar squaredmass matrix,

$$
\mathcal{M}^{2}=v^{2}\left(\begin{array}{ccc}
Z_{1} & \operatorname{Re}\left(Z_{6} e^{-i \eta}\right) & -\operatorname{Im}\left(Z_{6} e^{-i \eta}\right) \\
\operatorname{Re}\left(Z_{6} e^{-i \eta}\right) & \frac{1}{2}\left[Z_{34}+\operatorname{Re}\left(Z_{5} e^{-2 i \eta}\right)\right]+Y_{2} / v^{2} & -\frac{1}{2} \operatorname{Im}\left(Z_{5} e^{-2 i \eta}\right) \\
-\operatorname{Im}\left(Z_{6} e^{-i \eta}\right) & -\frac{1}{2} \operatorname{Im}\left(Z_{5} e^{-2 i \eta}\right) & \frac{1}{2}\left[Z_{34}-\operatorname{Re}\left(Z_{5} e^{-2 i \eta}\right)\right]+Y_{2} / v^{2}
\end{array}\right)
$$

which is expressed with respect to the $\left\{\sqrt{2} \operatorname{Re} \mathcal{H}_{1}^{0}-v\right.$, $\left.\sqrt{2} \operatorname{Re} \mathcal{H}_{2}^{0}, \sqrt{2} \operatorname{Im} \mathcal{H}_{2}^{0}\right\}$ basis, where ${ }^{11}$

$Z_{34} \equiv Z_{3}+Z_{4}=\lambda\left[R+s_{2 \beta}^{2}(1-R)\right]-\lambda_{5}\left(1-2 s_{2 \beta}^{2} \sin ^{2} \xi\right)$, after making use of Eqs. (5.16) and (5.17). The eigenvalues of $\mathcal{M}^{2}$ are independent of the choice of $\eta$, since these cannot depend on the phase choice used in the definition of the Higgs basis field $\mathcal{H}_{2}$. Hence, in practical calculations, one can choose $\eta$ to facilitate the analysis.

For example, if we choose $\eta=-\xi$, then the neutral scalar squared-mass matrix is given by

$$
\mathcal{M}^{2}=v^{2}\left(\begin{array}{ccc}
\lambda-L s_{2 \beta}^{2} & -L s_{2 \beta} c_{2 \beta} & -\frac{1}{2} \lambda_{5} s_{2 \beta} \sin 2 \xi \\
-L s_{2 \beta} c_{2 \beta} & \frac{2 \operatorname{Re}\left(m_{12}^{2} e^{i \xi}\right)}{v^{2} s_{2 \beta}}+L s_{2 \beta}^{2} & -\frac{1}{2} \lambda_{5} c_{2 \beta} \sin 2 \xi \\
-\frac{1}{2} \lambda_{5} s_{2 \beta} \sin 2 \xi & -\frac{1}{2} \lambda_{5} c_{2 \beta} \sin 2 \xi & \frac{2 \operatorname{Re}\left(m_{12}^{2} e^{i \xi}\right)}{v^{2} s_{2 \beta}}-\lambda_{5} \cos 2 \xi
\end{array}\right)
$$

where

$$
L \equiv \frac{1}{2} \lambda(1-R)+\lambda_{5} \sin ^{2} \xi
$$

\footnotetext{
${ }^{11}$ The expressions given for $m_{H^{ \pm}}^{2}$ in Eq. (5.21) and for $\mathcal{M}^{2}$ in Eqs. (5.22) and (5.23) in terms of the Higgs basis parameters are valid for the most general $2 \mathrm{HDM}$ scalar potential.
}

If $Z_{6} \neq 0$ and/or $Z_{7} \neq 0$ then the neutral scalar squaredmass matrix has a block diagonal form consisting of a $2 \times 2$ block and a $1 \times 1$ block if and only if $\operatorname{Im}\left(Z_{5} e^{-2 i \eta}\right)=0$ and $\operatorname{Re}\left(Z_{6} e^{-i \eta}\right) \operatorname{Im}\left(Z_{6} e^{-i \eta}\right)=0$. In such cases, the scalar potential and vacuum are $C P$ conserving, and we shall employ the following convention for the names of the neutral scalar mass eigenstates: the $C P$-even scalars whose squared masses are the eigenvalues of the $2 \times 2$ block will be denoted by $h$ and $H$ where $m_{h} \leq m_{H}$, and the $1 \times 1$ block will be identified with the squared mass of the $C P$-odd scalar, $A$. 


\section{B. The softly broken $\mathbb{Z}_{2} \otimes \Pi_{2}$-symmetric scalar potential with one vanishing vev}

In the case where one of the vevs vanishes (i.e., $s_{2 \beta}=0$ ), Eqs. (2.12) and (2.13) imply that $m_{12}^{2}=0$. For example, if $v_{1}=v$ and $v_{2}=0$ then $Y_{2}$ is a free parameter, $Z_{i}=\lambda_{i}$, $c_{2 \beta}=1$, and $\xi$ is indeterminate. In particular, $Y_{3}=Z_{6}=$ $Z_{7}=0$, which signals the presence of a $\mathbb{Z}_{2}$ symmetry, $\mathcal{H}_{1} \rightarrow+\mathcal{H}_{1}, \mathcal{H}_{2} \rightarrow-\mathcal{H}_{2}$, that is not broken by the vacuum. This is a special case of the IDM and corresponds to the inert limit of the softly broken $\mathbb{Z}_{2} \otimes \Pi_{2}$-symmetric scalar potential. In particular, $Y_{2}=m_{22}^{2}$ is a free parameter that is generically not equal to $Y_{1}=m_{11}^{2}=-\frac{1}{2} \lambda v^{2}$. To obtain the neutral scalar squared-mass matrix from Eq. (5.22), we must make a choice of $\eta$. For reasons discussed below Eq. (8.60), we shall choose $e^{-2 i \eta}=-1$. The neutral scalar squared-mass matrix is then diagonal and we may identify

$$
\begin{gathered}
m_{A}^{2}=Y_{2}+\frac{1}{2} \lambda v^{2} R, \\
m_{H^{ \pm}}^{2}=Y_{2}+\frac{1}{2} Z_{3} v^{2}=m_{A}^{2}-\frac{1}{2}\left(\lambda_{4}+\lambda_{5}\right) v^{2}, \\
m_{h}^{2}=\lambda v^{2}, \\
m_{H}^{2}=m_{A}^{2}-\lambda_{5} v^{2},
\end{gathered}
$$

where we have denoted the mass-eigenstate neutral scalar fields in the inert limit by

$h \equiv \sqrt{2} \operatorname{Re} \mathcal{H}_{1}^{0}-v, \quad H \equiv \sqrt{2} \operatorname{Re} \mathcal{H}_{2}^{0}, \quad A \equiv \sqrt{2} \operatorname{Im} \mathcal{H}_{2}^{0}$.

This nomenclature (where no mass ordering is implied) will be employed in all subsequent occurrences of the inert limit in this work, and differs from the convention adopted in the paragraph following Eq. (5.25) for the $C P$-conserving case where $Z_{6} \neq 0$ and/or $Z_{7} \neq 0$.

If $v_{1}=0$ and $v_{2}=v$, then one transforms to the Higgs

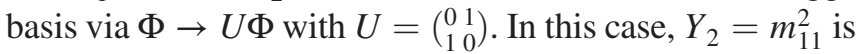
a free parameter, $Y_{1}=m_{22}^{2}=-\frac{1}{2} \lambda v^{2}, Y_{3}=Z_{6}=Z_{7}=0$, $Z_{i}=\lambda_{i}, c_{2 \beta}=-1$, and $\xi=0$. The scalar squared masses are again given by Eqs. (5.26)-(5.29).

In the inert limit where $Y_{3}=Z_{6}=Z_{7}=0$, the scalar potential and vacuum are automatically $C P$ conserving. In particular, in the inert limit the neutral scalars consist of a $C P$-even neutral scalar $h$ whose properties coincide with those of the SM Higgs boson and two neutral scalars $H$ and $A$ with opposite sign $C P$-quantum numbers. However, one cannot separately assign unique $C P$-quantum numbers to $H$ and $A$, respectively, based on the interactions of the scalars with the gauge bosons and the scalar self-interactions. ${ }^{12}$

\footnotetext{
${ }^{12}$ Indeed, the choice of $e^{-2 i \eta}=1$ would have interchanged the identities of $H$ and $A$ in Eqs. (5.26)-(5.29).
}

$C P$-conserving interactions of the scalars with other sectors of the theory, if present, will often resolve the ambiguity and identify $A$ as the neutral $C P$-odd scalar of the inert scalar doublet.

For example, the most general form for $C P$-conserving neutral Higgs interactions with one generation of fermions in the inert limit is obtained by setting $q_{11}=1, q_{22}=1$, $q_{32}=i$ (with all other $q_{k j}=0$ ), $\rho^{D *}=\rho^{D}$, and $\rho^{U *}=\rho^{U}$ in Eq. (58) of Ref. [37], which yields ${ }^{13}$

$$
\begin{aligned}
-\mathcal{L}_{\mathrm{Y}}= & \frac{1}{v}\left(m_{d} \bar{d} d+m_{u} \bar{u} u\right) h+\frac{1}{\sqrt{2}}\left(\rho^{D} \bar{d} d+\rho^{U} \bar{u} u\right) H \\
& +\frac{i}{\sqrt{2}}\left(\rho^{D} \bar{d} \gamma_{5} d-\rho^{U} \bar{u} \gamma_{5} u\right) A
\end{aligned}
$$

indicating that $h$ behaves like the SM Higgs boson, $H$ is $C P$ even and $A$ is $C P$ odd. Note that $\rho^{D}=\rho^{U}=0$ in the IDM, since $\mathcal{H}_{2}$ is the only $\mathbb{Z}_{2}$-odd field of the model, in which case the individual $C P$-quantum numbers of $H$ and $A$ are not resolved.

\section{Noncoexistence of an inert phase and a mixed phase}

Let us examine more closely when a vacuum in which one of the two vevs vanishes can arise. Here, we shall extend the analysis of Ref. [70] to the case of $\lambda_{5} \neq 0$. First, we require that $R>-1$ due to Eq. (5.1). If $v_{1}=v$ and $v_{2}=0$, then Eq. (2.13) yields $m_{12}^{2}=0$ and $m_{11}^{2}=-\frac{1}{2} \lambda v^{2}<0$. The value of the scalar potential at the minimum is $V_{\min }=-\left(m_{11}^{2}\right)^{2} /\left(2 \lambda^{2}\right)$. The positivity of $m_{A}^{2}$ given in Eq. (5.29) yields $m_{22}^{2}+\frac{1}{2} \lambda R v^{2}>0$. Hence, it follows that

$$
m_{22}^{2}>R m_{11}^{2}
$$

The above inequality is equivalent to

$$
(1+R)\left(m_{11}^{2}-m_{22}^{2}\right)<(1-R)\left(m_{11}^{2}+m_{22}^{2}\right) .
$$

Since $1+R$ is always positive, it follows that

$$
m_{22}^{2}-m_{11}^{2}>-\left(\frac{1-R}{1+R}\right)\left(m_{11}^{2}+m_{22}^{2}\right)
$$

In the case of $v_{1}=0$ and $v_{2}=v$, the roles of $m_{11}^{2}$ and $m_{22}^{2}$ are interchanged. That is,

\footnotetext{
${ }^{13}$ Introducing Yukawa interactions constitutes a hard breaking of the symmetries responsible for the ERPS. Thus, in this paper we shall not entertain such terms further.
} 


$$
m_{22}^{2}-m_{11}^{2}<\left(\frac{1-R}{1+R}\right)\left(m_{11}^{2}+m_{22}^{2}\right)
$$

Although the vanishing of one of the two vevs requires that $m_{12}^{2}=0$, the converse is not necessarily true. If $m_{12}^{2}=0$ then two different phases of the 2HDM are possible-an inert phase where one of the two vevs vanishes and a mixed phase where both vevs are nonzero. To analyze the latter possibility in more detail, we again extend the analysis presented in Ref. [70] to the case of $\lambda_{5} \neq 0$. If $m_{12}^{2}=0$ and $v_{1}, v_{2} \neq 0$, then Eqs. (2.4)-(2.7) yield

$$
\begin{gathered}
m_{11}^{2}=-\frac{1}{2} \lambda\left(v_{1}^{2}+R v_{2}^{2}\right), \\
m_{22}^{2}=-\frac{1}{2} \lambda\left(v_{2}^{2}+R v_{1}^{2}\right), \\
0=\lambda_{5} \sin 2 \xi .
\end{gathered}
$$

Since $\lambda_{5} \neq 0$ by assumption, it follows that $\sin 2 \xi=0$ and $\cos 2 \xi= \pm 1$. One is always free to rephase one of the scalar doublet fields so that $\xi=0$, since the only possible effect on the scalar potential parameters is a sign change of $\lambda_{5}$. In the convention where $\xi=0$, Eq. (5.24) yields $m_{A}^{2}=-\lambda_{5} v^{2}$, which implies that $\lambda_{5}<0$. Equation (2.11) then yields

$$
V_{\min }=-\frac{1}{8} \lambda\left(v_{1}^{4}+v_{2}^{4}+2 R v_{1}^{2} v_{2}^{2}\right)
$$

It is convenient to eliminate $v_{1}$ and $v_{2}$ in favor of the scalar potential parameters. Using Eqs. (5.36) and (5.37), one easily obtains

$$
\begin{aligned}
& v_{1}^{2}=\frac{2}{\lambda}\left(\frac{m_{22}^{2} R-m_{11}^{2}}{1-R^{2}}\right), \\
& v_{2}^{2}=\frac{2}{\lambda}\left(\frac{m_{11}^{2} R-m_{22}^{2}}{1-R^{2}}\right) .
\end{aligned}
$$

Plugging these values into Eq. (5.39) yields

$$
\begin{aligned}
V_{\min } & =-\frac{1}{2 \lambda\left(1-R^{2}\right)}\left[m_{11}^{4}+m_{22}^{4}-2 R m_{11}^{2} m_{22}^{2}\right] \\
& =-\frac{1}{4 \lambda}\left[\frac{\left(m_{11}^{2}+m_{22}^{2}\right)^{2}}{1+R}+\frac{\left(m_{11}^{2}-m_{22}^{2}\right)^{2}}{1-R}\right] .
\end{aligned}
$$

One can work out a number of inequalities that must be satisfied if the mixed phase is stable. We again require that $R>-1$ in light of Eq. (2.2). Using Eq. (5.24) with $m_{12}^{2}=\xi=0$, the trace and determinant of the $2 \times 2$ neutral $C P$-even scalar squared-mass matrix yield

$$
m_{h}^{2}+m_{H}^{2}=\lambda v^{2}, \quad m_{h}^{2} m_{H}^{2}=\frac{1}{4} \lambda^{2} v^{4} s_{2 \beta}^{2}\left(1-R^{2}\right) .
$$

Hence, the positivity of the scalar squared masses implies that $|R|<1$.

Next, we employ Eqs. (5.36) and (5.37) along with $|R|<1$ to obtain

$$
\begin{gathered}
m_{11}^{2}+m_{22}^{2}=-\frac{1}{2} \lambda v^{2}(1+R)<0, \\
m_{11}^{2}+m_{22}^{2}+\lambda v^{2}=\frac{1}{2} \lambda v^{2}(1-R)>0 .
\end{gathered}
$$

Using Eq. (5.4) and $\left|c_{2 \beta}\right| \leq 1$, it follows that $m_{11}^{2} \geq-\frac{1}{2} \lambda v^{2}$ and $m_{22}^{2} \geq-\frac{1}{2} \lambda v^{2}$. However, it is again more useful to provide inequalities that are independent of the vevs. In light of Eq. (5.40), the requirement that $v_{1}^{2}$ and $v_{2}^{2}$ are strictly positive implies that

$$
m_{22}^{2} R>m_{11}^{2}, \quad m_{11}^{2} R>m_{22}^{2} .
$$

The above inequalities are equivalent to

$$
\begin{gathered}
\left(\frac{1-R}{1+R}\right)\left(m_{11}^{2}+m_{22}^{2}\right)<m_{22}^{2}-m_{11}^{2} \\
<-\left(\frac{1-R}{1+R}\right)\left(m_{11}^{2}+m_{22}^{2}\right) .
\end{gathered}
$$

Comparing Eq. (5.46) with Eqs. (5.34) and (5.35), it follows that the mixed phase and the inert phase do not coexist $[57,70,71]$.

\section{D. $C P$ properties of the softly broken}

\section{$\mathbb{Z}_{2} \otimes \Pi_{2}$-symmetric scalar potential with $m_{12}^{2} \neq 0$}

Returning to the more general case where $m_{12}^{2} \neq 0$, the scalar sector is $C P$ conserving if and only if $\operatorname{Im}\left(Z_{5}^{*} Z_{6}^{2}\right)=0$. A straightforward computation yields

$$
\begin{gathered}
\operatorname{Im}\left(Z_{5}^{*} Z_{6}^{2}\right)=-\frac{1}{4} \lambda \lambda_{5}(1-R) s_{2 \beta}^{2} c_{2 \beta} \sin 2 \xi\left[\lambda(1-R)+2 \lambda_{5}\right] \\
=-\frac{1}{4} \lambda_{5}\left(\lambda-\lambda_{3}-\lambda_{4}-\lambda_{5}\right)\left(\lambda-\lambda_{3}-\lambda_{4}+\lambda_{5}\right) \\
\quad \times s_{2 \beta}^{2} c_{2 \beta} \sin 2 \xi .
\end{gathered}
$$

The case of $s_{2 \beta}=0$ corresponds to the inert limit, which has already been treated above. In light of Eq. (4.44), the conditions $\lambda_{5}=0$ and $\lambda-\lambda_{3}-\lambda_{4}= \pm \lambda_{5}$ correspond to the ERPS4 where a U(1) symmetry is manifestly realized in some basis. In particular, $\lambda=\lambda_{3}+\lambda_{4} \pm \lambda_{5}$ correspond to GCP3 and GCP3', respectively, whereas $\lambda_{5}=0$ corresponds to $\mathrm{U}(1) \otimes \Pi_{2}$, which is equivalent to GCP3 and GCP3' in different choices of the scalar field basis. For example, GCP3' is related to GCP3 via the basis change specified in Eq. (3.1). These enhanced symmetry cases will be treated separately in Secs. VI-VIII. 
In this section, we shall assume that $s_{2 \beta} \neq 0, \lambda \neq$ $\lambda_{3}+\lambda_{4} \pm \lambda_{5}$ and $\lambda_{5} \neq 0$, in which case $C P$ is conserved if either (or both) of the following two conditions hold:

$$
c_{2 \beta}=0 \quad \text { and } / \text { or } \quad \sin 2 \xi=0 .
$$

\section{1. $\cos 2 \beta=0$}

In the case of $c_{2 \beta}=0$, it follows that $C P$ is conserved despite the fact that one cannot separately rephase $\Phi_{1}$ and $\Phi_{2}$ in the $\mathbb{Z}_{2} \otimes \Pi_{2}$ basis such that all the parameters of the scalar potential are real if $\left.\operatorname{Im}\left(\lambda_{5}^{*}\left[m_{12}^{2}\right]^{2}\right]\right) \neq 0$, as was already noticed in Ref. [37]. To understand the origin of this result, note that Eq. (5.4) implies that $m_{11}^{2}=m_{22}^{2}$ when $c_{2 \beta}=0$. Together with the ERPS4 conditions, it follows that the scalar potential is invariant with respect to a GCP1'

$$
\mathcal{M}_{H}^{2}=\left(\begin{array}{cc}
\frac{1}{2} \lambda v^{2}(1+R)-\lambda_{5} v^{2} \sin ^{2} \xi & -\lambda_{5} v^{2} \sin \xi \cos \xi \\
-\lambda_{5} v^{2} \sin \xi \cos \xi & m_{A}^{2}-\frac{1}{2} \lambda v^{2}(1-R)-\lambda_{5} v^{2} \cos ^{2} \xi
\end{array}\right)
$$

with respect to the $\left\{\varphi_{1}, \varphi_{3}\right\}$ basis, where $\varphi_{1} \equiv \sqrt{2} \operatorname{Re} \mathcal{H}_{1}^{0}-v$ and $\varphi_{3} \equiv \sqrt{2} \operatorname{Im} \mathcal{H}_{2}^{0}$. The neutral $C P$-even scalar mass eigenstates are given by

$$
H=\varphi_{1} c_{\beta-\alpha}-\varphi_{3} s_{\beta-\alpha}, \quad h=\varphi_{1} s_{\beta-\alpha}+\varphi_{3} c_{\beta-\alpha},
$$

where $0 \leq \beta-\alpha \leq \pi, s_{\beta-\alpha} \equiv \sin (\beta-\alpha)$ and $c_{\beta-\alpha} \equiv \cos (\beta-\alpha)$,

$$
\begin{aligned}
m_{H, h}^{2}= & \frac{1}{2}\left\{m_{A}^{2}+\left(\lambda R-\lambda_{5}\right) v^{2}\right. \\
& \left. \pm \sqrt{\left[m_{A}^{2}-v^{2}\left(\lambda+\lambda_{5} \cos 2 \xi\right)\right]^{2}+\lambda_{5}^{2} v^{4} \sin ^{2} 2 \xi}\right\},
\end{aligned}
$$

with $m_{h} \leq m_{H}$, and ${ }^{14}$

$$
c_{\beta-\alpha}=\frac{\lambda_{5} v^{2} \sin 2 \xi}{2 \sqrt{\left(m_{H}^{2}-m_{h}^{2}\right)\left[m_{H}^{2}-\frac{1}{2} \lambda v^{2}(1+R)+\lambda_{5} v^{2} \sin ^{2} \xi\right]}} .
$$

\section{2. $\sin 2 \xi=0$}

In the case of $\sin 2 \xi=0$, Eqs. (5.5) and (5.6) imply that $\operatorname{Im}\left[\left(m_{12}^{2}\right)^{2}\right]=2 \operatorname{Re} m_{12}^{2} \operatorname{Im} m_{12}^{2}=0$. If $\sin \xi=0$ then $\operatorname{Im} m_{12}^{2}=0$ and all scalar potential parameters are real, whereas if $\cos \xi=0$ then $\operatorname{Re} m_{12}^{2}=0$ and a rephasing

\footnotetext{
${ }^{14}$ In obtaining Eq. (5.54), we have employed Eqs. (9.1) and (9.5), where the real quantity $Z_{6} v^{2}$ in these equations is to be identified with the off-diagonal element of $\mathcal{M}_{H}^{2}$ given in Eq. (5.51).
}

transformation (cf. Tables $\mathrm{V}$ and VI). Moreover, the condition of $c_{2 \beta}=0$ ensures that the $\mathrm{GCP}^{\prime}{ }^{\prime}$ symmetry is preserved by the vacuum.

When $c_{2 \beta}=0$, Eq. (5.24) is rendered block diagonal, with the $2 \times 2$ block identified as the squared-mass matrix of the neutral $C P$-even scalars. It then follows that

$$
\begin{array}{r}
m_{A}^{2}=2 \operatorname{Re}\left(m_{12}^{2} e^{i \xi}\right)+\frac{1}{2} \lambda v^{2}(1-R)+\lambda_{5} v^{2} \sin ^{2} \xi, \\
m_{H^{ \pm}}^{2}=m_{A}^{2}-\frac{1}{2} v^{2}\left[\lambda(1-R)+\lambda_{4}+\lambda_{5}\right],
\end{array}
$$

where $A=\varphi_{2} \equiv \sqrt{2} \operatorname{Re} \mathcal{H}_{2}^{0}$. The squared-mass matrix of the neutral $C P$-even scalars is

$\Phi_{2} \rightarrow i \Phi_{2}$ changes the sign of the real parameter $\lambda_{5}$ while removing the complex phase of $m_{12}^{2}$. Hence a real basis exists, ${ }^{15}$ which implies that the scalar potential and the vacuum are $C P$ conserving. The neutral scalar squared-mass matrix given in Eq. (5.24) is block diagonal when $\sin 2 \xi=0$, with the 33 element identified as the squared mass of the $C P$-odd scalar, $A=\varphi_{3} \equiv \sqrt{2} \operatorname{Im} \mathcal{H}_{2}^{0}$.

For $\sin \xi=0$, it follows from Eqs. (5.21) and (5.24) that

$$
\begin{gathered}
m_{A}^{2}= \pm \frac{2 \operatorname{Re} m_{12}^{2}}{s_{2 \beta}}-\lambda_{5} v^{2} \\
m_{H^{ \pm}}^{2}=m_{A}^{2}+\frac{1}{2}\left(\lambda_{5}-\lambda_{4}\right) v^{2}
\end{gathered}
$$

where the choice of signs corresponds to $\cos \xi= \pm 1$. The upper $2 \times 2$ block of Eq. (5.24) is identified as the squaredmass matrix of the $C P$-even neutral scalars,

$\mathcal{M}_{H}^{2}=\left(\begin{array}{cc}\lambda v^{2}\left[1-\frac{1}{2} s_{2 \beta}^{2}(1-R)\right] & -\frac{1}{2} \lambda v^{2} s_{2 \beta} c_{2 \beta}(1-R) \\ -\frac{1}{2} \lambda v^{2} s_{2 \beta} c_{2 \beta}(1-R) & m_{A}^{2}+\lambda_{5} v^{2}+\frac{1}{2} \lambda v^{2} s_{2 \beta}^{2}(1-R)\end{array}\right)$,

with respect to the $\left\{\varphi_{1}, \varphi_{2}\right\}$ basis, where $\varphi_{1} \equiv \sqrt{2} \operatorname{Re} \mathcal{H}_{1}^{0}-v$ and $\varphi_{2} \equiv \sqrt{2} \operatorname{Re} \mathcal{H}_{2}^{0}$. Hence,

\footnotetext{
${ }^{15} \mathrm{~A}$ real basis is defined to be a scalar field basis in which the scalar potential parameters and the vevs are simultaneously real.
} 


$$
m_{H, h}^{2}=\frac{1}{2}\left\{m_{A}^{2}+\left(\lambda+\lambda_{5}\right) v^{2} \pm \sqrt{\left[m_{A}^{2}+\lambda_{5} v^{2}-\lambda v^{2}\left(c_{2 \beta}^{2}+R s_{2 \beta}^{2}\right)\right]^{2}+\lambda^{2} s_{2 \beta}^{2} c_{2 \beta}^{2}(1-R)^{2} v^{4}}\right\}
$$

with $m_{h} \leq m_{H}$, and

$$
c_{\beta-\alpha}=\frac{\lambda v^{2} s_{2 \beta} c_{2 \beta}(1-R)}{2 \sqrt{\left(m_{H}^{2}-m_{h}^{2}\right)\left[m_{H}^{2}-\lambda v^{2}\left(1-\frac{1}{2} s_{2 \beta}^{2}(1-R)\right)\right]}} .
$$

For $\cos \xi=0$, the results of Eqs. (5.55)-(5.58) are modified by the following substitutions:

$$
\begin{aligned}
\pm \operatorname{Re} m_{12}^{2} & \rightarrow \mp \operatorname{Im} m_{12}^{2}, \quad \lambda_{5} \rightarrow-\lambda_{5}, \\
R & \rightarrow \bar{R} \equiv\left(\lambda_{3}+\lambda_{4}-\lambda_{5}\right) / \lambda,
\end{aligned}
$$

where the choice of signs in front of $\operatorname{Im} m_{12}^{2}$ corresponds to $\sin \xi= \pm 1$. Note that $\bar{R} \neq 1$ under the assumption specified above Eq. (5.48). If $\bar{R}=1$ then the (softly broken) $\mathbb{Z}_{2} \otimes \Pi_{2}$ symmetry of the scalar potential is promoted to GCP3', as discussed below Eq. (5.47).

\section{3. $\cos 2 \beta=\sin 2 \xi=0$}

If $c_{2 \beta}=\sin 2 \xi=0$ then Eqs. (5.19) and (5.20) yield $Y_{3}=Z_{6}=Z_{7}=0$, corresponding to an inert limit of the softly broken $\mathbb{Z}_{2} \otimes \Pi_{2}$-symmetric scalar potential. If $\sin \xi=0$, then one can obtain the scalar squared masses either by taking the $\sin \xi=\operatorname{Im} m_{12}^{2}=0$ limit of Eqs. (5.49)(5.51) or by taking the $c_{2 \beta}=0$ limit of Eqs. (5.55)-(5.57). Recall that we have identified the neutral scalar mass eigenstates in the convention specified in Eq. (5.30). Taking into account that $\mathcal{M}_{H}^{2}$ is exhibited with respect to the $\left\{\varphi_{1}, \varphi_{3}\right\}$ basis in Eq. (5.51) and with respect to the $\left\{\varphi_{1}, \varphi_{2}\right\}$ basis in Eq. (5.56), respectively, it follows that

$$
\begin{aligned}
m_{h}^{2} & =\frac{1}{2} \lambda v^{2}(1+R), \quad m_{A}^{2}= \pm 2 \operatorname{Re} m_{12}^{2}-\lambda_{5} v^{2}, \\
m_{H}^{2} & =m_{A}^{2}+\lambda_{5} v^{2}+\frac{1}{2} \lambda v^{2}(1-R), \\
m_{H^{ \pm}}^{2} & =m_{A}^{2}+\frac{1}{2}\left(\lambda_{5}-\lambda_{4}\right) v^{2} .
\end{aligned}
$$

If $\cos \xi=0$, then Eq. (5.60) is modified by applying the substitutions indicated in Eq. (5.59). In the inert limit, the vacuum preserves the $\Pi_{2}$ symmetry (whereas the $\mathbb{Z}_{2}$ symmetry remains softly broken since $m_{12}^{2} \neq 0$ ).

\section{Spontaneous vs explicit CP violation}

Spontaneous $C P$ violation can occur when $\operatorname{Im}\left[m_{12}^{2}\right]^{2}=0$ (with $m_{12}^{2} \neq 0$ ) and $\sin 2 \xi \neq 0$. In addition, as noted in Appendix A below Eq. (A25), one must assume that $\lambda_{5}>0$ in order to guarantee that this $C P$-violating vacuum solution is a local minimum. If $\operatorname{Im} m_{12}^{2}=0$ and $\sin 2 \xi \neq 0$, then Eq. (5.6) implies that $m_{11}^{2}+m_{22}^{2}+$ $\frac{1}{2} \lambda(1+R) v^{2}=\lambda_{5} v^{2}$. Inserting this result into Eq. (5.5) yields $\cos \xi=\operatorname{Re} m_{12}^{2} /\left(\lambda_{5} v^{2} s_{\beta} c_{\beta}\right)$; i.e., spontaneous $C P$ violation occurs if [42]

$$
0<\left|m_{12}^{2}\right|<\lambda_{5} v^{2} s_{\beta} c_{\beta}
$$

Likewise, if $\operatorname{Re} m_{12}^{2}=0$ and $\sin 2 \xi \neq 0$, then Eq. (5.5) implies that $m_{11}^{2}+m_{22}^{2}+\frac{1}{2} \lambda(1+R) v^{2}=0$. Inserting this result into Eq. (5.6) yields $\sin \xi=\operatorname{Im} m_{12}^{2} /\left(\lambda_{5} v^{2} s_{\beta} c_{\beta}\right)$. Once again, spontaneous $C P$ violation occurs if Eq. (5.61) is satisfied.

If $\operatorname{Im}\left(Z_{5}^{*} Z_{6}^{2}\right) \neq 0$ then the scalar potential is explicitly $C P$ violating. In this case, one must diagonalize the $3 \times 3$ neutral scalar squared-mass matrix given in Eq. (5.24) to determine the neutral scalar mass eigenstates. The inert limit cannot be realized in this case, so the presence of scalar-mediated $C P$-violating effects necessarily implies that the tree-level properties of any of the three neutral scalar mass eigenstates must deviate from those of the SM Higgs boson. This is a special case of the C2HDM that has been explored in Ref. [37].

\section{E. Scalar potential with an unbroken $\mathbb{Z}_{2} \otimes \Pi_{2}$-symmetry}

The $\mathbb{Z}_{2} \otimes \Pi_{2}$ symmetry of the scalar potential is unbroken if $m_{11}^{2}=m_{22}^{2}$ and $m_{12}^{2}=0$. First, we suppose that both vevs are nonzero. Then Eq. (5.13) implies that $Y_{3}=0$, which yields $Y_{3}=Z_{6}=Z_{7}=0$ in light of Eq. (5.20) and the ERPS condition, corresponding to the inert limit of the $\mathbb{Z}_{2} \otimes \Pi_{2}$-symmetric scalar potential. Moreover in light of Eqs. (2.10) and (5.4), $c_{2 \beta}=\sin 2 \xi=0$ in the $\mathbb{Z}_{2} \otimes \Pi_{2}$ symmetry limit, and it follows that the vacuum breaks $\mathbb{Z}_{2}$ but conserves $\Pi_{2}$. The scalar squared masses in the limit of $c_{2 \beta}=\sin \xi=0$ and $m_{12}^{2}=0$ are given by the $m_{12}^{2}=0$ limit of Eq. (5.60). Stability of the scalar potential requires that the squared masses of the scalars are positive, which yields $\lambda_{5}=-\left|\lambda_{5}\right|<0, \lambda_{4}<\left|\lambda_{5}\right|$ and $|R|<1$. Likewise, for $c_{2 \beta}=\cos \xi=0$, the scalar squared masses are given by $m_{12}^{2}=0$ limit of Eq. (5.60) after replacing $\lambda_{5} \rightarrow-\lambda_{5}$ and $R \rightarrow \bar{R}$ [cf. Eq. (5.59)], in which case the stability requirement yields $\lambda_{5}>0, \lambda_{4}<\lambda_{5}$ and $|\bar{R}|<1$.

If one of the vevs vanishes (i.e., $s_{2 \beta}=0$ ) then the $\mathbb{Z}_{2} \otimes \Pi_{2}$ symmetry limit of Eq. (5.26) corresponds to setting $Y_{2}=Y_{1}=-\frac{1}{2} \lambda v^{2}$ [cf. Eqs. (2.12) and (2.13)], which yields 
TABLE VII. Landscape of the ERPS4-Part I: Scalar potentials of the 2 HDM with either an unbroken or softly broken $\mathbb{Z}_{2} \otimes \Pi_{2}$ symmetry that is manifestly realized in the $\Phi$-basis. In all cases, $\lambda \equiv \lambda_{1}=\lambda_{2} \neq \lambda_{3}+\lambda_{4} \pm \lambda_{5}$, where $\lambda_{5}$ is real and nonzero, $\lambda_{6}=\lambda_{7}=0$, and $\lambda, \lambda+\lambda_{3}$, and $\lambda+\lambda_{3}+\lambda_{4}-\left|\lambda_{5}\right|$ are all positive. Note that if $m_{12}^{2}$ is pure imaginary, one can rephase $\Phi_{2} \rightarrow i \Phi_{2}$ to obtain a new basis where $m_{12}^{2}$ is real and $\lambda_{5}$ flips sign. An exact Higgs alignment in the ERPS4 is realized in the inert limit where $Y_{3}=Z_{6}=Z_{7}=0$.

\begin{tabular}{|c|c|c|c|c|c|c|}
\hline$\beta$ & $\sin 2 \xi$ & $m_{11}^{2}, m_{22}^{2}$ & $m_{12}^{2}$ & $C P$ violation? & Higgs alignment & Comment \\
\hline$s_{2 \beta} \neq 0$ & $\neq 0$ & $m_{11}^{2} \neq m_{22}^{2}$ & Complex & Explicit & No & $\operatorname{Im}\left[m_{12}^{2}\right]^{2} \neq 0$ \\
\hline$s_{2 \beta} \neq 0$ & $\neq 0$ & $m_{11}^{2} \neq m_{22}^{2}$ & $\operatorname{Im}\left[m_{12}^{2}\right]^{2}=0$ & Spontaneous & No & $0<\left|m_{12}^{2}\right|<\frac{1}{2} \lambda_{5} v^{2} s_{2 \beta}$ \\
\hline$s_{2 \beta} \neq 0$ & $\neq 0$ & $m_{11}^{2} \neq m_{22}^{2}$ & $\operatorname{Im}\left[m_{12}^{2}\right]^{2}=0$ & No & No & $\left|m_{12}^{2}\right|>\frac{1}{2} \lambda_{5} v^{2} s_{2 \beta}$ \\
\hline$c_{2 \beta}=0$ & $\neq 0$ & $m_{11}^{2}=m_{22}^{2}$ & Complex & No & No & $m_{12}^{2} \neq 0$ \\
\hline$s_{2 \beta} c_{2 \beta} \neq 0$ & 0 & $m_{11}^{2} \neq m_{22}^{2}$ & $\operatorname{Im}\left[m_{12}^{2}\right]^{2}=0$ & No & No & \\
\hline$s_{2 \beta}=0$ & & $m_{11}^{2} \neq m_{22}^{2}$ & 0 & No & Yes & \\
\hline$c_{2 \beta}=0$ & 0 & $m_{11}^{2}=m_{22}^{2}$ & $\operatorname{Im}\left[m_{12}^{2}\right]^{2}=0$ & No & Yes & $m_{12}^{2} \neq 0$ \\
\hline$s_{2 \beta}=0$ & & $m_{11}^{2}=m_{22}^{2}$ & 0 & No & Yes & Unbroken $\mathbb{Z}_{2} \otimes \Pi_{2}$ \\
\hline$c_{2 \beta}=0$ & 0 & $m_{11}^{2}=m_{22}^{2}$ & 0 & No & Yes & Unbroken $\mathbb{Z}_{2} \otimes \Pi_{2}$ \\
\hline
\end{tabular}

$$
m_{A}^{2}=\frac{1}{2} \lambda v^{2}(R-1)
$$

which requires that $R>1$. The squared masses of $H^{ \pm}, h$ and $H$ in terms of $m_{A}^{2}$ given in Eqs. (5.27)-(5.29) remain unchanged. In this case, the vacuum breaks $\Pi_{2}$ but conserves $\mathbb{Z}_{2}$.

\section{F. The landscape of ERPS4-Part I: Scalar potential with a softly broken or unbroken $\mathbb{Z}_{2} \otimes \Pi_{2}$ symmetry}

The landscape of scalar potentials in the ERPS4 that respects a softly broken or exact $\mathbb{Z}_{2} \otimes \Pi_{2}$ symmetry (but no larger symmetry) is summarized in Table VII.

\section{THE U(1) $\otimes \Pi_{2}$ SCALAR FIELD BASIS}

Consider the softly broken $\mathrm{U}(1) \otimes \Pi_{2}$-symmetric scalar potential where $\lambda \equiv \lambda_{1}=\lambda_{2}$ and $\lambda_{5}=\lambda_{6}=\lambda_{7}=0$. The softly broken parameters, $m_{11}^{2}, m_{22}^{2}$ and $m_{12}^{2}$, are arbitrary with $m_{12}^{2}$ potentially complex. If we demand that the potential is bounded from below, the following conditions must be satisfied (modulo the remarks of footnote 3 ),

$$
\lambda>0, \quad \lambda+\lambda_{3}>0, \quad \lambda+\lambda_{3}+\lambda_{4}>0 .
$$

\section{A. The softly broken $U(1) \otimes \Pi_{2}$ or $S O(3)$-symmetric scalar potential with $v_{1} \neq 0$ and $v_{2} \neq 0$}

We shall first assume that $v_{1}$ and $v_{2}$ are both nonzero, or equivalently $\sin 2 \beta \neq 0$. We then use Eqs. (2.8)-(2.10) (with $\lambda \equiv \lambda_{1}=\lambda_{2}$ ) to obtain

$$
\begin{gathered}
m_{11}^{2}=\operatorname{Re}\left(m_{12}^{2} e^{i \xi}\right) \tan \beta-\frac{1}{2} \lambda v^{2} c_{\beta}^{2}-\frac{1}{2}\left(\lambda_{3}+\lambda_{4}\right) v^{2} s_{\beta}^{2} \\
m_{22}^{2}=\operatorname{Re}\left(m_{12}^{2} e^{i \xi}\right) \cot \beta-\frac{1}{2} \lambda v^{2} s_{\beta}^{2}-\frac{1}{2}\left(\lambda_{3}+\lambda_{4}\right) v^{2} c_{\beta}^{2} \\
\operatorname{Im}\left(m_{12}^{2} e^{i \xi}\right)=0 .
\end{gathered}
$$

Equations (6.2) and (6.3) fix the value of $\beta$. In particular,

$$
c_{2 \beta}=\frac{m_{22}^{2}-m_{11}^{2}}{m_{11}^{2}+m_{22}^{2}+\lambda v^{2}} .
$$

Since $m_{12}^{2}$ is the only potentially complex parameter, one can assume without loss of generality that $m_{12}^{2}$ is real and non-negative after an appropriate rephasing of one of the two Higgs doublet fields. Hence, Eq. (6.4) implies that the scalar sector is $C P$ conserving. Nevertheless, in the analysis presented in this section, we find it convenient to retain all factors of $e^{i \xi}$ for later purposes, which simply means that $m_{12}^{2} e^{i \xi}=\operatorname{Re}\left(m_{12}^{2} e^{i \xi}\right) \geq 0$, in light of Eq. (6.4) and the requirement that $m_{A}^{2} \geq 0$ [cf. Eq. (6.14)].

The corresponding parameters of the Higgs basis are obtained by setting $\lambda_{5}=0$ in Eqs. (5.14)-(5.19),

$$
\begin{gathered}
Y_{2}=\frac{2 \operatorname{Re}\left(m_{12}^{2} e^{i \xi}\right)}{s_{2 \beta}}-\frac{1}{2} \lambda v^{2}\left[R+\frac{1}{2} s_{2 \beta}^{2}(1-R)\right], \\
Z_{1}=Z_{2}=\lambda\left[1-\frac{1}{2} s_{2 \beta}^{2}(1-R)\right], \\
Z_{3}=\lambda_{3}+\frac{1}{2} \lambda s_{2 \beta}^{2}(1-R), \\
Z_{4}=\lambda_{4}+\frac{1}{2} \lambda s_{2 \beta}^{2}(1-R), \\
Z_{5}=\frac{1}{2} \lambda s_{2 \beta}^{2}(1-R) e^{-2 i \xi}, \\
Z_{6}=-Z_{7}=-\frac{1}{2} \lambda s_{2 \beta} c_{2 \beta}(1-R) e^{-i \xi},
\end{gathered}
$$

where

$$
R \equiv \frac{\lambda_{3}+\lambda_{4}}{\lambda}
$$


Note that $R>-1$ in light of Eq. (6.1). ${ }^{16}$ The limit of $R=1$ corresponds to the softly broken $\mathrm{SO}(3)$-symmetric scalar potential, where the conditions $\lambda_{1}=\lambda_{2}=\lambda_{3}+\lambda_{4}$ and $\lambda_{5}=$ $\lambda_{6}=\lambda_{7}=0$ hold for all choices of the scalar field basis.

The Higgs basis parameters $Y_{1}$ and $Y_{3}$ are fixed by the potential minimum conditions given in Eq. (5.20). Note that $\operatorname{Im}\left(Z_{5}^{*} Z_{6}^{2}\right)=0$, which implies that a real Higgs basis exists after an appropriate rephasing of the Higgs basis field $\mathcal{H}_{2}$. That is, there is no $C P$ violation (neither explicit nor spontaneous) arising from a scalar potential that exhibits a softly broken $\mathrm{U}(1) \otimes \Pi_{2}$ symmetry. Using Eqs. (6.7)(6.11), it follows that the following conditions are satisfied:

$$
\begin{gathered}
{\left[\operatorname{Re}\left(Z_{5}^{*} Z_{6}^{2}\right)\right]^{2}+\operatorname{Re}\left(Z_{5}^{*} Z_{6}^{2}\right)\left|Z_{6}\right|^{2}\left(Z_{1}-Z_{34}\right)-2\left|Z_{6}\right|^{6}=0} \\
\text { and } \operatorname{Im}\left(Z_{5}^{*} Z_{6}^{2}\right)=0 .
\end{gathered}
$$

We recognize these conditions as equivalent to Eq. (4.41) when applied in the Higgs basis.

The squared masses of the neutral Higgs bosons are obtained by computing the eigenvalues of Eq. (5.22). In light of Eqs. (6.10) and (6.11), it is convenient to take $\eta=-\xi$ in Eq. (5.22), since this choice yields $\operatorname{Im}\left(Z_{5} e^{-2 i \eta}\right)=\operatorname{Im}\left(Z_{6} e^{-i \eta}\right)=0$. One can then immediately identity the squared mass of the $C P$-odd neutral scalar $A=\varphi_{3} \equiv \sqrt{2} \operatorname{Im} \mathcal{H}_{2}^{0}$,

$$
m_{A}^{2}=\frac{1}{2} v^{2}\left[Z_{34}-\operatorname{Re}\left(Z_{5} e^{2 i \xi}\right)\right]+Y_{2}=\frac{2 \operatorname{Re}\left(m_{12}^{2} e^{i \xi}\right)}{s_{2 \beta}} .
$$

Combining Eqs. (6.2), (6.3) and (6.14) yields an alternative expression,

$$
m_{A}^{2}=m_{11}^{2}+m_{22}^{2}+\frac{1}{2} \lambda v^{2}(1+R) .
$$

Likewise, the charged Higgs squared mass is given by

$$
m_{H^{ \pm}}^{2}=Y_{2}+\frac{1}{2} Z_{3} v^{2}=m_{A}^{2}-\frac{1}{2} \lambda_{4} v^{2}
$$

after making use of Eq. (6.14). Finally, the squared masses of the $C P$-even neutral scalars, denoted by $h$ and $H$, are the eigenvalues of the $2 \times 2$ matrix,

$$
\begin{aligned}
\mathcal{M}_{H}^{2} & =\left(\begin{array}{cc}
Z_{1} v^{2} & \operatorname{Re}\left(Z_{6} e^{i \xi}\right) v^{2} \\
\operatorname{Re}\left(Z_{6} e^{i \xi}\right) v^{2} & m_{A}^{2}+\operatorname{Re}\left(Z_{5} e^{2 i \xi}\right) v^{2}
\end{array}\right) \\
& =\left(\begin{array}{cc}
\lambda v^{2}\left[1-\frac{1}{2} s_{2 \beta}^{2}(1-R)\right] & -\frac{1}{2} \lambda v^{2} s_{2 \beta} c_{2 \beta}(1-R) \\
-\frac{1}{2} \lambda v^{2} s_{2 \beta} c_{2 \beta}(1-R) & m_{A}^{2}+\frac{1}{2} \lambda v^{2} s_{2 \beta}^{2}(1-R)
\end{array}\right),
\end{aligned}
$$

with respect to the $\left\{\varphi_{1}, \varphi_{2}\right\}$ basis, ${ }^{17}$ where $\varphi_{1} \equiv$ $\sqrt{2} \operatorname{Re} \mathcal{H}_{1}^{0}-v$ and $\varphi_{2} \equiv \sqrt{2} \operatorname{Re} \mathcal{H}_{2}^{0}$. The neutral $C P$-even scalar masses are given by

$$
m_{H, h}^{2}=\frac{1}{2}\left\{m_{A}^{2}+\lambda v^{2} \pm \sqrt{\left[m_{A}^{2}-\lambda v^{2}\left(c_{2 \beta}^{2}+R s_{2 \beta}^{2}\right)\right]^{2}+\lambda^{2} s_{2 \beta}^{2} c_{2 \beta}^{2}(1-R)^{2} v^{4}}\right\}
$$

with $m_{h} \leq m_{H}$, and

$$
c_{\beta-\alpha}=\frac{\lambda v^{2} s_{2 \beta} c_{2 \beta}(1-R)}{2 \sqrt{\left(m_{H}^{2}-m_{h}^{2}\right)\left[m_{H}^{2}-\lambda v^{2}\left(1-\frac{1}{2} s_{2 \beta}^{2}(1-R)\right)\right]}} .
$$

A stable minimum requires that the scalar squared masses should be non-negative. This condition implies that

$$
\operatorname{Re}\left(m_{12}^{2} e^{i \xi}\right) \geq 0 \quad \text { and } \quad m_{A}^{2} \geq \frac{1}{2} \lambda_{4} v^{2}
$$

\footnotetext{
${ }^{16}$ If $R=-1$ then the quartic terms of the scalar potential exhibit a flat direction. One can then ensure the stability of the scalar potential in the weak sense (cf. footnote 3) if $m_{11}^{2}+m_{22}^{2}>$ $2\left|m_{12}^{2}\right|[56,72]$.

${ }^{17}$ The computation of the squared-mass matrix of the $C P$-even neutral scalars in the $\Phi$-basis is given in Appendix D.
}

In addition, we demand that

$$
\begin{gathered}
\operatorname{Tr} \mathcal{M}_{H}^{2}=m_{A}^{2}+\lambda v^{2} \geq 0 \\
\frac{1}{v^{2}} \operatorname{det} \mathcal{M}_{H}^{2}=\frac{1}{4} \lambda^{2} v^{2} s_{2 \beta}^{2}\left(1-R^{2}\right)+\lambda m_{A}^{2}\left[1-\frac{1}{2} s_{2 \beta}^{2}(1-R)\right] \\
\geq 0 .
\end{gathered}
$$

Since $m_{A}^{2} \geq 0$ by assumption, Eq. (6.21) is automatically satisfied in light of Eq. (6.1). On the other hand, Eq. (6.22) is satisfied only if $R$ lies below a critical positive value that depends on $\lambda, \beta$ and $m_{A}^{2} / v^{2}$,

$$
-1<R \leq \frac{m_{A}^{2}}{\lambda v^{2}}+\sqrt{\left(\frac{m_{A}^{2}}{\lambda v^{2}}-1\right)^{2}+\frac{4 m_{A}^{2}}{\lambda v^{2} s_{2 \beta}^{2}},}
$$


after employing Eq. (6.1). ${ }^{18}$ It follows that Eq. (6.22) is satisfied for all values of $\beta$ if

$$
-1<R \leq 1+\frac{2 m_{A}^{2}}{\lambda v^{2}}
$$

One can fix the parameter space of the softly broken $\mathrm{U}(1) \otimes \Pi_{2}$ scalar potential by specifying the values of six real parameters: $\lambda, \lambda_{4}, R, \beta, m_{A}$ and $v=246 \mathrm{GeV}$. By replacing $\lambda$ with $m_{h}$ [see Eq. (131) of Ref. [70] ] and $\lambda_{4}$ with $m_{H^{ \pm}}$, the independent parameters of the softly broken $\mathrm{U}(1) \otimes \Pi_{2}$ scalar potential can be taken as $m_{h}, m_{A}$, $m_{H^{ \pm}}, v, R$ and $\beta$, in which case $m_{H}^{2}=m_{A}^{2}-m_{h}^{2}+\lambda v^{2}$ [cf. Eq. (6.21)] is a derived quantity.

\section{B. The inert limit of the softly broken $U(1) \otimes \Pi_{2}$ or SO(3)-symmetric scalar potential}

The inert limit of the scalar potential, where $Y_{3}=Z_{6}=$ $Z_{7}=0$, possesses an exact $\mathbb{Z}_{2}$ symmetry despite the presence of squared mass parameters that softly break the $\mathrm{U}(1) \otimes \Pi_{2}$ symmetry. The inert limit arises if either $v_{1}=0$ or $v_{2}=0$, but is more general. Indeed, Eqs. (2.2) and (6.11) imply that the inert limit arises if any one of the three conditions, $R=1, c_{2 \beta}=0$, or $s_{2 \beta}=0$, is satisfied.

\section{Softly broken $S O(3)$-symmetric scalar potential $(R=1)$}

The case of $R=1$ corresponds to the softly broken $\mathrm{SO}(3)$ scalar potential as noted below Eq. (6.12). In light of Eqs. (6.2)-(6.4), it follows that if $s_{2 \beta} c_{2 \beta} \neq 0$ then $m_{11}^{2} \neq m_{22}^{2}$ and $\operatorname{Re}\left(m_{12}^{2} e^{i \xi}\right) \neq 0$. In this case, the squared masses of the Higgs bosons are given by

$m_{h}^{2}=\lambda v^{2}, \quad m_{H}^{2}=m_{A}^{2}, \quad m_{H^{ \pm}}^{2}=m_{A}^{2}-\frac{1}{2} \lambda_{4} v^{2}$,

where $m_{A}^{2}=2 \operatorname{Re}\left(m_{12}^{2} e^{i \xi}\right) / s_{2 \beta}$. The mass degeneracy of $H$ and $A$ arises due to an unbroken U(1) symmetry of the scalar potential in the Higgs basis (since $Y_{3}=Z_{5}=$ $Z_{6}=Z_{7}=0$ ) that is preserved by the vacuum. The $\Pi_{2}$ symmetry remains softly broken (since $Y_{1} \neq Y_{2}$ ).

\section{The softly broken $\mathrm{U}(1) \otimes \Pi_{2}$-symmetric scalar potential with $\cos 2 \beta=0$}

In the case of $c_{2 \beta}=0$, Eq. (6.5) implies that $m_{11}^{2}=m_{22}^{2}$. Equations (6.14)-(6.18) yield

\footnotetext{
${ }^{18}$ Apart from the upper bound given in Eq. (6.23), one can obtain an independent upper bound by imposing either tree-level unitarity [73-77] or a perturbativity constraint. One would then expect $R /(4 \pi) \lesssim \mathcal{O}(1)$.
}

$$
\begin{aligned}
m_{h}^{2} & =\frac{1}{2} \lambda v^{2}(1+R), \quad m_{H}^{2}=m_{A}^{2}+\frac{1}{2} \lambda v^{2}(1-R), \\
m_{H^{ \pm}}^{2} & =m_{A}^{2}-\frac{1}{2} \lambda_{4} v^{2},
\end{aligned}
$$

where $m_{A}^{2}=2 \operatorname{Re}\left(m_{12}^{2} e^{i \xi}\right)$, in agreement with the $\lambda_{5}=0$ limit of Eq. (5.60). In this limiting case, after rephasing one of the two Higgs doublet fields to set $\xi=0$, the vacuum preserves the $\Pi_{2}$ symmetry [whereas the U(1) symmetry remains softly broken since $m_{12}^{2} \neq 0$ ].

\section{The softly broken $\mathrm{U}(\mathbf{1}) \otimes \Pi_{2}$-symmetric scalar potential with one vanishing vev}

The case where one of the vevs vanishes (i.e., $s_{2 \beta}=0$ ) should be treated separately and implies that $m_{12}^{2}=0$ in light of Eqs. (2.12) and (2.13). One can check that Eqs. (6.7)-(6.11) remain valid after setting $s_{2 \beta}=0$. In this case, the $\mathrm{U}(1)$ symmetry of the scalar potential is unbroken, whereas the $\Pi_{2}$ symmetry is softly broken if $m_{11}^{2} \neq m_{22}^{2}$.

First, suppose that $v_{2}=0$ and $v_{1}=v$. Then, Eq. (5.26) yields

$$
m_{A}^{2}=Y_{2}+\frac{1}{2} \lambda v^{2} R
$$

where $Y_{2}$ is a free parameter of the scalar potential that is no longer given by Eq. (6.6). Moreover, Eq. (6.5) is no longer valid since $Y_{2}=m_{22}^{2}$ is independent of the squared mass parameter $m_{11}^{2}$; only the latter is fixed by the scalar potential minimum condition. The squared masses of the other scalars are given by Eqs. (5.27)-(5.29) by setting $\lambda_{5}=0$,

$m_{h}^{2}=\lambda v^{2}, \quad m_{H}^{2}=m_{A}^{2}, \quad m_{H^{ \pm}}^{2}=m_{A}^{2}-\frac{1}{2} \lambda_{4} v^{2}$.

Note that the U(1) symmetry is preserved by the vacuum, which results in the mass degeneracy of $H$ and $A$. Second, if $v_{1}=0$ and $v_{2}=v$, then it follows that $Y_{2}=m_{11}^{2}$ is a free parameter and $Y_{1}=m_{22}^{2}=-\frac{1}{2} \lambda v^{2}$. Equations (6.7)-(6.11) remain valid after setting $\beta=\frac{1}{2} \pi$. Moreover, the Higgs masses given by Eqs. (6.27) and (6.28) also remain valid.

\section{The mixed phase of the softly broken}

\section{$\mathrm{U}(1) \otimes \Pi_{2}$-symmetric scalar potential with $m_{12}^{2}=0$}

Although the vanishing of one of the two vevs requires that $m_{12}^{2}=0$, the converse is not necessarily true, as previously noted. That is, if $m_{12}^{2}=0$, then both an inert phase and a mixed phase of the $2 \mathrm{HDM}$ are possible. The inequalities previously obtained that distinguish the inert and mixed phases in Eqs. (5.34), (5.35) and (5.46) still apply (after setting $\lambda_{5}=0$ ), and again imply that the inert and mixed phases do not coexist. In the mixed phase with $m_{12}^{2}=0$, the scalar potential respects the $\mathrm{U}(1)$ symmetry, which is spontaneously broken by the vacuum. 
Consequently, $m_{A}^{2}=0$ and the other scalar squared masses are given by

$$
\begin{aligned}
& m_{H, h}^{2}=\frac{1}{2} \lambda v^{2}\left[1 \pm \sqrt{c_{2 \beta}^{2}+R^{2} s_{2 \beta}^{2}}\right] \\
& m_{H^{ \pm}}^{2}=-\frac{1}{2} \lambda_{4} v^{2},
\end{aligned}
$$

with $m_{h} \leq m_{H}$. Stability of the vacuum requires that $\lambda_{4}<0$.

\section{Scalar potential with an unbroken $U(1) \otimes \Pi_{2}$ or $\mathrm{SO}(3)$ symmetry}

The $\mathrm{U}(1) \otimes \Pi_{2}$ symmetry of the scalar potential is unbroken if $m_{11}^{2}=m_{22}^{2}$ and $m_{12}^{2}=0$. Then, as noted at the end of Sec. $\mathrm{V}$, the squared mass conditions yield $Y_{3}=Z_{6}=Z_{7}=0$, corresponding to the inert limit of the $\mathrm{U}(1) \otimes \Pi_{2}$-symmetric scalar potential.

First, we suppose that both vevs are nonzero. Then in the $\mathrm{U}(1) \otimes \Pi_{2}$ symmetry limit, Eqs. (6.2)-(6.4) imply that $(R-1) c_{2 \beta}=0$. Hence, the $\mathrm{U}(1) \otimes \Pi_{2}$ symmetry limit arises in two distinct cases. If $m_{12}^{2}=c_{2 \beta}=0$ and $R \neq 1$, then Eqs. (6.14)-(6.17) yield

$m_{h}^{2}=\frac{1}{2} \lambda v^{2}(1+R), \quad m_{H}^{2}=\frac{1}{2} \lambda v^{2}(1-R)$,

$m_{A}^{2}=0, \quad m_{H^{ \pm}}^{2}=-\frac{1}{2} \lambda_{4} v^{2}$.

Note that a stable minimum exists if $\lambda_{4}<0$ and $|R|<1$. The $\Pi_{2}$ symmetry is preserved by the vacuum, whereas the $\mathrm{U}(1)$ symmetry is spontaneously broken by the vacuum and results in a massless Goldstone boson.
If $m_{11}^{2}=m_{22}^{2}, m_{12}^{2}=0$ and $R=1$, then an $\mathrm{SO}(3)$ symmetry is explicitly preserved by the scalar potential and Eqs. (6.14)-(6.17) yield

$m_{h}^{2}=\lambda v^{2}, \quad m_{H}^{2}=m_{A}^{2}=0, \quad m_{H^{ \pm}}^{2}=-\frac{1}{2} \lambda_{4} v^{2}$.

The $\mathrm{SO}(3)$ symmetry is spontaneously broken by the vacuum, leaving a residual unbroken $U(1)$ symmetry, which results in two massless Goldstone bosons, $H$ and $A$.

If one of the vevs vanishes (i.e., $s_{2 \beta}=0$ ), then setting $\lambda_{5}=0$ in Eq. (5.62) and in Eqs. (5.27)-(5.29) yields

$$
\begin{aligned}
m_{h}^{2} & =\lambda v^{2}, \quad m_{H}^{2}=m_{A}^{2}=\frac{1}{2} \lambda v^{2}(R-1), \\
m_{H^{ \pm}}^{2} & =m_{A}^{2}-\frac{1}{2} \lambda_{4} v^{2},
\end{aligned}
$$

which corresponds to a stable minimum if $R>1$. Note that in this case the $\Pi_{2}$ symmetry is broken by the vacuum, whereas the $\mathrm{U}(1)$ symmetry is preserved by the vacuum and results in the mass degeneracy of $H$ and $A$. In the limit of $R=1$, corresponding to an $\mathrm{SO}(3)$-symmetric scalar potential, the resulting scalar masses are again given by Eq. (6.31).

\section{E. The landscape of ERPS4-Part II(a): Scalar potential with a softly broken or unbroken $\mathrm{U}(1) \otimes \Pi_{2}$ or $\mathbf{S O}(3)$ symmetry}

Table VIII provides a summary of the landscape of scalar potentials in the subspace of the ERPS4 regime where the $\mathrm{U}(1) \otimes \Pi_{2}$ or $\mathrm{SO}(3)$ symmetry of the scalar potential is either softly broken $\left(m_{11}^{2} \neq m_{22}^{2}\right.$ and/or $\left.m_{12}^{2} \neq 0\right)$ or unbroken $\left(m_{11}^{2}=m_{22}^{2}\right.$ and $\left.m_{12}^{2}=0\right)$.

TABLE VIII. Landscape of the ERPS4-Part II(a): Scalar potentials of the 2HDM with either an unbroken or softly broken U(1) $\otimes$ $\Pi_{2}$ symmetry that is manifestly realized in the $\Phi$-basis, where $\lambda \equiv \lambda_{1}=\lambda_{2}, \lambda_{5}=\lambda_{6}=\lambda_{7}=0$, and $C P$ is conserved by the scalar potential and vacuum. The parameter $m_{12}^{2} e^{i \xi}$ is real and non-negative [as a consequence of Eqs. (6.4) and (6.14)]; if $m_{12}^{2}=0$ and $s_{2 \beta} \neq 0$ then a massless neutral scalar is present in the neutral scalar spectrum. The parameter $R \equiv\left(\lambda_{3}+\lambda_{4}\right) / \lambda>-1$; when $R=1$ the (softly broken) $\mathrm{U}(1) \otimes \Pi_{2}$ symmetry is promoted to a (softly broken) $\mathrm{SO}(3)$ symmetry. An exact Higgs alignment in the ERPS4 is realized in the inert limit where $Y_{3}=Z_{6}=Z_{7}=0$.

\begin{tabular}{llcccc}
\hline \hline$\beta$ & $m_{11}^{2}, m_{22}^{2}$ & $m_{12}^{2} e^{i \xi}$ & $R$ & Higgs alignment & Comment \\
\hline$s_{2 \beta} c_{2 \beta} \neq 0$ & $m_{11}^{2} \neq m_{22}^{2}$ & $>0$ & $R \neq 1$ & No & See Eq. (6.23) \\
$s_{2 \beta} c_{2 \beta} \neq 0$ & $m_{11}^{2} \neq m_{22}^{2}$ & 0 & $|R|<1$ & No & $m_{A}^{2}=0$ \\
$c_{2 \beta}=0$ & $m_{11}^{2}=m_{22}^{2}$ & $>0$ & $R \neq 1$ & Yes & $-1<R \leq 1+2 m_{A}^{2} /\left(\lambda v^{2}\right)$ \\
$s_{2 \beta}=0$ & $m_{11}^{2} \neq m_{22}^{2}$ & 0 & $R \neq 1$ & Yes & $m_{H}^{2}=m_{A}^{2}>0$ \\
$c_{2 \beta}=0$ & $m_{11}^{2}=m_{22}^{2}$ & 0 & $|R|<1$ & Yes & One massless scalar \\
$s_{2 \beta}=0$ & $m_{11}^{2}=m_{22}^{2}$ & 0 & $R>1$ & Yes & $m_{H}^{2}=m_{A}^{2}>0$ \\
$s_{2 \beta} c_{2 \beta} \neq 0$ & $m_{11}^{2} \neq m_{22}^{2}$ & $>0$ & $R=1$ & Yes & $m_{H}^{2}=m_{A}^{2}>0$ \\
$c_{2 \beta}=0$ & $m_{11}^{2}=m_{22}^{2}$ & $>0$ & $R=1$ & Yes & $m_{H}^{2}=m_{A}^{2}>0$ \\
$s_{2 \beta}=0$ & $m_{11}^{2} \neq m_{22}^{2}$ & 0 & $R=1$ & Yes & $m_{H}^{2}=m_{A}^{2}>0$ \\
& $m_{11}^{2}=m_{22}^{2}$ & 0 & $R=1$ & Yes & $m_{H}^{2}=m_{A}^{2}=0$ \\
\hline \hline
\end{tabular}


It is noteworthy that the tree-level Higgs scalar potential of the MSSM exhibits a softly broken $U(1) \otimes \Pi_{2}$ symmetry with $m_{11}^{2} \neq m_{22}^{2}, \quad m_{12}^{2} \neq 0, \quad s_{2 \beta} \neq 0$ and $R=-1$ $[22,78]$, corresponding to the first line of Table VIII. ${ }^{19}$ Of course, radiative corrections to the scalar potential of the MSSM are significant [79] and yield an effective $2 \mathrm{HDM}$ scalar potential below the energy scale of supersymmetry breaking that lies outside the domain of the ERPS4 [80].

\section{THE GCP3 SCALAR FIELD BASIS}

Consider a softly broken GCP3 symmetric scalar potential whose parameters (denoted with prime superscripts) satisfy the following conditions: $\lambda^{\prime} \equiv \lambda_{1}^{\prime}=\lambda_{2}^{\prime}=\lambda_{3}^{\prime}+\lambda_{4}^{\prime}+\lambda_{5}^{\prime}$ and $\operatorname{Im} \lambda_{5}^{\prime}=\lambda_{6}^{\prime}=\lambda_{7}^{\prime}=0$. The softly broken parameters $m_{11}^{\prime 2}, m_{12}^{\prime 2}$ and $m_{12}^{\prime 2}$ are arbitrary (with $m_{12}^{\prime 2}$ potentially complex). If we demand that the potential is bounded from below, then

$$
\lambda^{\prime}>0, \quad \lambda^{\prime}+\lambda_{3}^{\prime}>0, \quad \lambda_{5}^{\prime}<\lambda^{\prime}
$$

\section{A. The softly broken GCP3 symmetric scalar potential with $v_{1} \neq 0$ and $v_{2} \neq 0$}

Assuming that $v_{1}^{\prime}$ and $v_{2}^{\prime}$ are both nonzero, Eqs. (2.8)(2.10) yield

$$
\begin{gathered}
m_{11}^{\prime 2}=\operatorname{Re}\left(m_{12}^{\prime 2} e^{i \xi^{\prime}}\right) \tan \beta^{\prime}-\frac{1}{2} \lambda^{\prime} v^{2}+\lambda_{5}^{\prime} v^{2} s_{\beta^{\prime}}^{2} \sin ^{2} \xi^{\prime}, \\
m_{22}^{\prime 2}=\operatorname{Re}\left(m_{12}^{\prime 2} e^{i \xi^{\prime}}\right) \cot \beta^{\prime}-\frac{1}{2} \lambda^{\prime} v^{2}+\lambda_{5}^{\prime} v^{2} c_{\beta^{\prime}}^{2} \sin ^{2} \xi^{\prime}, \\
\operatorname{Im}\left(m_{12}^{\prime 2} e^{i \xi^{\prime}}\right)=\frac{1}{2} \lambda_{5}^{\prime} v^{2} s_{\beta^{\prime}} c_{\beta^{\prime}} \sin 2 \xi^{\prime} .
\end{gathered}
$$

Equations (7.2)-(7.4) fix the value of $\beta^{\prime}$ and $\xi^{\prime}$. In particular,

$$
\begin{gathered}
c_{2 \beta^{\prime}}=\frac{m_{22}^{\prime 2}-m_{11}^{\prime 2}}{m_{11}^{\prime 2}+m_{22}^{\prime 2}+\lambda^{\prime} v^{2}}, \\
\cos \xi^{\prime}=\frac{2 \operatorname{Re} m_{12}^{\prime 2}}{s_{2 \beta^{\prime}}\left(m_{11}^{\prime 2}+m_{22}^{\prime 2}+\lambda^{\prime} v^{2}\right)}, \\
\sin \xi^{\prime}=\frac{-2 \operatorname{Im} m_{12}^{\prime 2}}{s_{2 \beta^{\prime}}\left[m_{11}^{\prime 2}+m_{22}^{\prime 2}+\left(\lambda^{\prime}-\lambda_{5}^{\prime}\right) v^{2}\right]} .
\end{gathered}
$$

\footnotetext{
${ }^{19}$ Since $R=-1$, the scalar potential stability conditions require that $m_{11}^{2}+m_{22}^{2}>2\left|m_{12}^{2}\right|$ as noted in footnote 16 . Moreover, $m_{11}^{2} m_{22}^{2}<\left|m_{12}^{2}\right|^{2}$ in order to have electroweak symmetry breaking [72], thereby excluding $m_{11}^{2}=m_{22}^{2}$.
}

As noted below Eq. (5.6), inserting $m_{12}^{\prime 2}=\left|m_{12}^{\prime 2}\right| e^{i \theta_{12}^{\prime}}$ in Eqs. (7.6) and (7.7) and imposing $\cos ^{2} \xi^{\prime}+\sin ^{2} \xi^{\prime}=1$ yields an equation that determines the phase $\theta_{12}^{\prime}$ in terms of $\xi^{\prime}$ and the other GCP3 scalar potential parameters.

The corresponding parameters of the Higgs basis are obtained by setting $R=1$ in Eqs. (5.14)-(5.19),

$$
\begin{gathered}
Y_{2}=\frac{2 \operatorname{Re}\left(m_{12}^{\prime 2} e^{i \xi^{\prime}}\right)}{s_{2 \beta^{\prime}}}-\frac{1}{2} \lambda^{\prime} v^{2}+\lambda_{5}^{\prime} v^{2}\left(1-\frac{1}{2} s_{2 \beta^{\prime}}^{2}\right) \sin ^{2} \xi^{\prime} \\
Z_{1}=Z_{2}=\lambda^{\prime}-\lambda_{5}^{\prime} s_{2 \beta^{\prime}}^{2} \sin ^{2} \xi^{\prime} \\
Z_{3}=\lambda_{3}^{\prime}+\lambda_{5}^{\prime} s_{2 \beta^{\prime}}^{2} \sin ^{2} \xi^{\prime} \\
Z_{4}=\lambda_{4}^{\prime}+\lambda_{5}^{\prime} s_{2 \beta^{\prime}}^{2} \sin ^{2} \xi^{\prime} \\
Z_{5}=\lambda_{5}^{\prime} e^{-2 i \xi^{\prime}}\left(\cos \xi^{\prime}+i c_{2 \beta^{\prime}} \sin \xi^{\prime}\right)^{2} \\
Z_{6}=-Z_{7}=i \lambda_{5}^{\prime} s_{2 \beta^{\prime}} \sin \xi^{\prime} e^{-i \xi^{\prime}}\left(\cos \xi^{\prime}+i c_{2 \beta^{\prime}} \sin \xi^{\prime}\right)
\end{gathered}
$$

The Higgs basis parameters $Y_{1}$ and $Y_{3}$ are fixed by the potential minimum conditions given in Eq. (5.20). Note that Eq. (6.13) is satisfied, as expected. In addition, in the limit of $\lambda_{5}^{\prime}=0$, we recover the softly broken $\mathrm{SO}(3)$ symmetric scalar potential, where the conditions $\lambda_{1}^{\prime}=\lambda_{2}^{\prime}=$ $\lambda_{3}^{\prime}+\lambda_{4}^{\prime}$ and $\lambda_{5}^{\prime}=\lambda_{6}^{\prime}=\lambda_{7}^{\prime}=0$ hold for all choices of the scalar field basis.

One can check that $C P$ is conserved in light of the relation,

$$
Z_{6}^{2}=-\lambda_{5}^{\prime} s_{2 \beta^{\prime}}^{2} \sin ^{2} \xi^{\prime} Z_{5},
$$

which implies that $\operatorname{Im}\left(Z_{5}^{*} Z_{6}^{2}\right)=0$. Thus, there exists an appropriate rephasing of the Higgs basis such that $Z_{5}, Z_{6}$ and $Z_{7}$ are real. This is remarkable in light of the fact that $\lambda_{5}$ is real but $m_{12}^{2}$ can be complex, which implies that one cannot perform a simple rephasing of the scalar doublet fields in the GCP3 basis to render all parameters real. In light of the $C P$ invariance of a softly broken GCP3-symmetric scalar potential, it must be possible to find a residual generalized $C P$ transformation under which the scalar potential and the vacuum in the GCP3 basis is left invariant. In Appendix C, we provide an explicit construction of this residual generalized $C P$ transformation. Of course, the existence of such a transformation is a foregone conclusion given that the existence of the residual $C P$ symmetry in the $\mathrm{U}(1) \otimes \Pi_{2}$ basis can be established by inspection.

The scalar masses can now be evaluated. First, Eq. (5.21) is still valid,

$$
\begin{aligned}
m_{H^{ \pm}}^{2} & =Y_{2}+\frac{1}{2} Z_{3} v^{2} \\
& =\frac{2 \operatorname{Re}\left(m_{12}^{\prime 2} e^{i \xi^{\prime}}\right)}{s_{2 \beta^{\prime}}}-\frac{1}{2} v^{2}\left(\lambda_{4}^{\prime}+\lambda_{5}^{\prime} \cos 2 \xi^{\prime}\right) .
\end{aligned}
$$


Next, consider the neutral scalar squared-mass matrix, which is given by Eq. (5.22). Noting that the complex number, $\cos \xi^{\prime}+i c_{2 \beta^{\prime}} \sin \xi^{\prime}$ appears in both Eqs. (7.12) and (7.13), it is convenient to define the complex phase $\psi$ via

$$
\cos \xi^{\prime}+i c_{2 \beta^{\prime}} \sin \xi^{\prime}=\left(1-s_{2 \beta^{\prime}}^{2} \sin ^{2} \xi^{\prime}\right)^{1 / 2} e^{i \psi} .
$$

In order to make use of Eq. (5.22), we must choose a value for $\eta$. Following Eq. (5.8), we shall transform $\eta=-\xi$ [which was employed in the $\mathrm{U}(1) \otimes \Pi_{2}$ basis] to the GCP3 basis. The derivation is provided in Sec. VIII [cf. Eqs. (8.58)-(8.60)] and instructs us to choose

$$
\eta=\psi-\xi^{\prime}-\frac{1}{2} \pi
$$

Inserting this result into Eq. (5.22), it then follows that

$$
\begin{gathered}
Z_{5}=-\lambda_{5}^{\prime}\left(1-s_{2 \beta^{\prime}}^{2} \sin ^{2} \xi^{\prime}\right) e^{2 i \eta} \\
Z_{6}=-\lambda_{5}^{\prime} s_{2 \beta^{\prime}} \sin \xi^{\prime}\left(1-s_{2 \beta^{\prime}}^{2} \sin ^{2} \xi^{\prime}\right)^{1 / 2} e^{i \eta} .
\end{gathered}
$$

In particular, $\operatorname{Im}\left(Z_{5} e^{-2 i \eta}\right)=\operatorname{Im}\left(Z_{6} e^{-i \eta}\right)=0$. Thus, we can immediately read off the squared mass of the $C P$-odd neutral scale from Eq. (5.22),

$$
\begin{aligned}
m_{A}^{2} & =Y_{2}+\frac{1}{2} v^{2}\left[Z_{3}+Z_{4}-\operatorname{Re}\left(Z_{5} e^{-2 i \eta}\right)\right] \\
& =\frac{2 \operatorname{Re}\left(m_{12}^{\prime 2} e^{i \xi^{\prime}}\right)}{s_{2 \beta^{\prime}}}+\lambda_{5}^{\prime} v^{2} \sin ^{2} \xi^{\prime}
\end{aligned}
$$

where $A=\varphi_{3} \equiv \sqrt{2} \operatorname{Im} \mathcal{H}_{2}^{0}$. Combining the results of Eqs. (7.2), (7.3) and (7.20) yields

$$
m_{A}^{2}=m_{11}^{\prime 2}+m_{22}^{\prime 2}+\lambda^{\prime} v^{2}
$$

In addition, Eqs. (7.15) and (7.20) yield

$$
m_{H^{ \pm}}^{2}=m_{A}^{2}-\frac{1}{2}\left(\lambda_{4}^{\prime}+\lambda_{5}^{\prime}\right) v^{2} .
$$

The squared masses of the $C P$-even neutral scalars, $h$ and $H$ are the eigenvalues of the $2 \times 2$ matrix exhibited below, ${ }^{20}$

$$
\begin{aligned}
\mathcal{M}_{H}^{2} & =\left(\begin{array}{cc}
Z_{1} v^{2} & -\operatorname{Im}\left(Z_{6} e^{i\left(\xi^{\prime}-\psi\right)}\right) v^{2} \\
-\operatorname{Im}\left(Z_{6} e^{i\left(\xi^{\prime}-\psi\right)}\right) v^{2} & m_{A}^{2}-\operatorname{Re}\left(Z_{5} e^{2 i\left(\xi^{\prime}-\psi\right)}\right) v^{2}
\end{array}\right) \\
& =\left(\begin{array}{cc}
\left(\lambda^{\prime}-\lambda_{5}^{\prime} s_{2 \beta^{\prime}}^{2} \sin ^{2} \xi^{\prime}\right) v^{2} & -\lambda_{5}^{\prime} v^{2} s_{2 \beta^{\prime}} \sin \xi^{\prime}\left(1-s_{2 \beta^{\prime}}^{2} \sin ^{2} \xi^{\prime}\right)^{1 / 2} \\
-\lambda_{5}^{\prime} v^{2} s_{2 \beta^{\prime}} \sin \xi^{\prime}\left(1-s_{2 \beta^{\prime}}^{2} \sin ^{2} \xi^{\prime}\right)^{1 / 2} & m_{A}^{2}-\lambda_{5}^{\prime} v^{2}\left(1-s_{2 \beta^{\prime}}^{2} \sin ^{2} \xi^{\prime}\right)
\end{array}\right),
\end{aligned}
$$

with respect to the $\left\{\varphi_{1}, \varphi_{2}\right\}$ basis, where $\varphi_{1} \equiv \sqrt{2} \operatorname{Re} \mathcal{H}_{1}^{0}-v$ and $\varphi_{2} \equiv \sqrt{2} \operatorname{Re} \mathcal{H}_{2}^{0}$. The neutral $C P$-even scalar masses are given by

$$
m_{H, h}^{2}=\frac{1}{2}\left\{m_{A}^{2}+\left(\lambda^{\prime}-\lambda_{5}^{\prime}\right) v^{2} \pm \sqrt{\left[m_{A}^{2}-\left(\lambda^{\prime}+\lambda_{5}^{\prime}\right) v^{2}\right]^{2}+4 \lambda_{5}^{\prime} v^{2}\left(m_{A}^{2}-\lambda^{\prime} v^{2}\right) s_{2 \beta^{\prime}}^{2} \sin ^{2} \xi^{\prime}}\right\}
$$

where $m_{h} \leq m_{H}$, and

$$
c_{\beta-\alpha}=\frac{\lambda_{5}^{\prime} v^{2} s_{2 \beta^{\prime}} \sin \xi^{\prime}\left(1-s_{2 \beta^{\prime}}^{2} \sin ^{2} \xi^{\prime}\right)^{1 / 2}}{\sqrt{\left(m_{H}^{2}-m_{h}^{2}\right)\left[m_{H}^{2}-\left(\lambda^{\prime}-\lambda_{5}^{\prime} s_{2 \beta^{\prime}}^{2} \sin ^{2} \xi^{\prime}\right) v^{2}\right]}} .
$$

A stable minimum requires that the scalar squared masses should be non-negative. This condition implies that

$$
\begin{aligned}
& \operatorname{Re}\left(m_{12}^{2} e^{i \xi^{\prime}}\right)+\frac{1}{2} \lambda_{5}^{\prime} v^{2} s_{2 \beta} \sin ^{2} \xi^{\prime} \geq 0 \\
& \text { and } m_{A}^{2} \geq \frac{1}{2}\left(\lambda_{4}^{\prime}+\lambda_{5}^{\prime}\right) v^{2}
\end{aligned}
$$

\footnotetext{
${ }^{20}$ The computation of the squared-mass matrix of the $C P$-even neutral scalars starting from the $\Phi^{\prime}$-basis is much more difficult. Details are provided in Appendix D.
}

In addition, we demand that

$$
\begin{gathered}
\operatorname{Tr} \mathcal{M}_{H}^{2}=m_{A}^{2}+\left(\lambda^{\prime}-\lambda_{5}^{\prime}\right) v^{2} \geq 0, \\
\frac{1}{v^{2}} \operatorname{det} \mathcal{M}_{H}^{2}=m_{A}^{2}\left(\lambda^{\prime}-\lambda_{5}^{\prime} s_{2 \beta^{\prime}}^{2} \sin ^{2} \xi^{\prime}\right)-\lambda^{\prime} \lambda_{5}^{\prime} v^{2}\left(1-s_{2 \beta^{\prime}}^{2} \sin ^{2} \xi^{\prime}\right)
\end{gathered}
$$$$
\geq 0 \text {. }
$$

Since $m_{A}^{2} \geq 0$ by assumption, Eq. (7.27) is automatically satisfied in light of Eq. (7.1). On the other hand, Eq. (7.28) is satisfied if and only if

$$
\lambda_{5}^{\prime} \leq \min \left\{\lambda^{\prime}, \frac{\lambda^{\prime} m_{A}^{2}}{\lambda^{\prime} v^{2}+s_{2 \beta^{\prime}}^{2} \sin ^{2} \xi^{\prime}\left(m_{A}^{2}-\lambda^{\prime} v^{2}\right)}\right\} .
$$

One can fix the parameter space of the softly broken GCP3 scalar potential by specifying the values of $\lambda^{\prime}, \lambda_{4}^{\prime}, \lambda_{5}^{\prime}$, 
$s_{2 \beta^{\prime}} \sin \xi^{\prime}, m_{A}$ and $v$. In particular, once $m_{A}$ is fixed, we see that $\beta^{\prime}$ and $\xi^{\prime}$ do not appear independently in any 2HDM observable. By replacing $\lambda$ with $m_{h}$ and $\lambda_{4}$ with $m_{H^{ \pm}}$, the independent parameters of the softly broken GCP3 scalar potential can be taken to be $m_{h}, m_{A}, m_{H^{ \pm}}, v, \lambda_{5}^{\prime}$ and $s_{2 \beta^{\prime}} \sin \xi^{\prime}$. That is, just as in the case of the softly broken $\mathrm{U}(1) \otimes \Pi_{2}$ scalar potential, the parameter space is fixed by six real parameters.

\section{B. The inert limit of the softly broken GCP3-symmetric scalar potential}

The inert limit of the softly broken GCP3-symmetric, corresponding to $Y_{3}=Z_{6}=Z_{7}=0$, arises if either $v_{1}=0$ or $v_{2}=0$, but is more general. Indeed, Eq. (7.13) implies that the inert limit requires that one of the following three conditions, $s_{2 \beta^{\prime}} \sin \xi^{\prime}=0, c_{2 \beta^{\prime}}=\cos \xi^{\prime}=0$, or $\lambda_{5}^{\prime}=0$, is satisfied.

\section{The softly broken GCP3-symmetric scalar potential with one vanishing vev}

The case where one of the vevs vanishes (i.e., $s_{2 \beta^{\prime}}=0$ ) implies that $m_{12}^{\prime 2}=0$ in light of Eqs. (2.12) and (2.13). Then, setting $R=1$ in Eqs. (5.26)-(5.29) yields

$$
\begin{aligned}
m_{h}^{2} & =\lambda^{\prime} v^{2}, \quad m_{H}^{2}=m_{A}^{2}-\lambda_{5}^{\prime} v^{2}, \\
m_{H^{ \pm}}^{2} & =m_{A}^{2}-\frac{1}{2}\left(\lambda_{4}^{\prime}+\lambda_{5}^{\prime}\right) v^{2},
\end{aligned}
$$

where $m_{A}^{2}=Y_{2}+\frac{1}{2} \lambda^{\prime} v^{2}$ and $Y_{2}$ is a free parameter. Likewise, if $\sin \xi^{\prime}=0$, then Eqs. (7.20)-(7.23) also yield Eq. (7.30), where $m_{A}^{2}=2\left|\operatorname{Re} m_{12}^{\prime 2}\right| / s_{2 \beta^{\prime}} .{ }^{21}$ That is, if $s_{2 \beta^{\prime}} \sin \xi^{\prime}=0$ then Eq. (7.30) is satisfied where

$m_{A}^{2}= \begin{cases}\frac{2\left|\operatorname{Re} m_{12}^{\prime 2}\right|}{s_{2 \beta^{\prime}}}, & \text { if } \sin \xi^{\prime}=0 \text { and } s_{2 \beta^{\prime}} \neq 0, \\ Y_{2}+\frac{1}{2} \lambda^{\prime} v^{2}, & \text { if } s_{2 \beta^{\prime}}=0 .\end{cases}$

Note that if $\sin \xi^{\prime}=0, s_{2 \beta^{\prime}} c_{2 \beta^{\prime}} \neq 0$ and $m_{11}^{\prime 2} \neq m_{22}^{\prime 2}$, then it follows that $\operatorname{Re} m_{12}^{\prime 2} \neq 0$ in light of Eqs. (7.2)-(7.4).

\section{The softly broken GCP3-symmetric scalar potential with $\cos 2 \beta^{\prime}=\cos \xi^{\prime}=0$}

Second, if $c_{2 \beta^{\prime}}=\cos \xi^{\prime}=0$, then it follows from Eqs. (7.20)-(7.23) that

$$
\begin{aligned}
m_{h}^{2} & =\left(\lambda^{\prime}-\lambda_{5}^{\prime}\right) v^{2}, \quad m_{H}^{2}=m_{A}^{2}= \pm 2 \operatorname{Im} m_{12}^{\prime 2}+\lambda_{5}^{\prime} v^{2}, \\
m_{H^{ \pm}}^{2} & =m_{A}^{2}-\frac{1}{2}\left(\lambda_{4}^{\prime}+\lambda_{5}^{\prime}\right) v^{2} .
\end{aligned}
$$

\footnotetext{
${ }^{21}$ If $\sin \xi^{\prime}=0$ then $\operatorname{Re}\left(m_{12}^{\prime 2} e^{i \xi^{\prime}}\right)= \pm \operatorname{Re} m_{12}^{\prime 2}=\left|\operatorname{Re} m_{12}^{\prime 2}\right|$ after choosing the sign that yields $m_{A}^{2} \geq 0$.
}

Using the results of Sec. VIII, this case corresponds to $s_{2 \beta}=0$ in the $\mathrm{U}(1) \otimes \Pi_{2}$ basis. Then the choice of plus (minus) sign in the expression for $m_{H, A}^{2}$ in Eq. (7.32) corresponds to $\beta=0 \quad\left(\beta=\frac{1}{2} \pi\right)$, respectively. Moreover, recall that an unbroken GCP3 symmetry is equivalent to $\mathrm{U}(1)^{\prime} \otimes \mathbb{Z}_{2}$ (cf. Table VI). Although the $\mathbb{Z}_{2}$ symmetry is explicitly broken (due to $m_{12}^{\prime 2} \neq 0$ ), a residual $\mathrm{U}(1)^{\prime}$ symmetry survives that is preserved by the vacuum if $c_{2 \beta^{\prime}}=\cos \xi^{\prime}=0$ since

$$
\left(\begin{array}{cc}
\cos \theta & \sin \theta \\
-\sin \theta & \cos \theta
\end{array}\right)\left(\begin{array}{c}
1 \\
\pm i
\end{array}\right)=e^{ \pm i \theta}\left(\begin{array}{c}
1 \\
\pm i
\end{array}\right)
$$

which results in the mass degeneracy of $H$ and $A$.

\section{Softly broken SO(3)-symmetric scalar potential $\left(\lambda_{5}^{\prime}=0\right)$}

Third, if $\lambda_{5}^{\prime}=0$, then the softly broken GCP3 symmetry is promoted to a softly broken $\mathrm{SO}(3)$ symmetry. In light of Eqs. (7.2)-(7.4), it follows that if $s_{2 \beta^{\prime}} c_{2 \beta^{\prime}} \neq 0$ then $m_{11}^{\prime 2} \neq m_{22}^{\prime 2}$ and $\operatorname{Re}\left(m_{12}^{\prime 2} e^{i \xi^{\prime}}\right) \neq 0$. We then obtain,

$$
\begin{aligned}
m_{h}^{2} & =\lambda^{\prime} v^{2}, \quad m_{H}^{2}=m_{A}^{2}=\frac{2 \operatorname{Re}\left(m_{12}^{\prime 2} e^{i \xi^{\prime}}\right)}{s_{2 \beta^{\prime}}}, \\
m_{H^{ \pm}}^{2} & =m_{A}^{2}-\frac{1}{2} \lambda_{4}^{\prime} v^{2},
\end{aligned}
$$

in agreement with Eq. (6.25), as expected. Using the results of Sec. VIII, this case corresponds to $R=1$ in the $\mathrm{U}(1) \otimes \Pi_{2}$ basis.

\section{Scalar potential with an unbroken GCP3 symmetry}

Finally, if $m_{11}^{\prime 2}=m_{22}^{\prime 2}$ and $m_{12}^{\prime 2}=0$ then the GCP3 symmetry is explicitly preserved by the scalar potential. In light of Eqs. (5.13) and (5.20) and the ERPS condition, it follows that $Y_{3}=Z_{6}=Z_{7}=0$, corresponding to the inert limit of the scalar potential. If both vevs are nonzero, then it follows from Eqs. (7.2)-(7.4) that $\lambda_{5}^{\prime} c_{2 \beta^{\prime}} \sin ^{2} \xi^{\prime}=$ $\lambda_{5}^{\prime} \sin \xi^{\prime} \cos \xi^{\prime}=0$. Consequently, the GCP3 symmetry limit arises in the following three distinct cases.

First, if $\lambda_{5}^{\prime} \neq 0$ and $\sin \xi^{\prime}=0$, then Eqs. (7.30) and (7.31) yield

$$
\begin{aligned}
& m_{h}^{2}=\lambda^{\prime} v^{2}, \quad m_{H}^{2}=-\lambda_{5}^{\prime} v^{2}, \\
& m_{A}^{2}=0, \quad m_{H^{ \pm}}^{2}=-\frac{1}{2}\left(\lambda_{4}^{\prime}+\lambda_{5}^{\prime}\right) v^{2},
\end{aligned}
$$

which corresponds to a stable minimum if $\lambda_{5}^{\prime}<0$ and $\lambda_{4}^{\prime}<-\lambda_{5}^{\prime}$. The GCP3 symmetry is spontaneously broken by the vacuum, resulting in a massless scalar.

Second, if $\lambda_{5}^{\prime} \neq 0, \quad c_{2 \beta^{\prime}}=0$ and $\cos \xi^{\prime}=0$, then Eq. (7.32) yields 


$$
\begin{aligned}
m_{h}^{2} & =\left(\lambda^{\prime}-\lambda_{5}^{\prime}\right) v^{2}, \quad m_{H}^{2}=m_{A}^{2}=\lambda_{5}^{\prime} v^{2}, \\
m_{H^{ \pm}}^{2} & =\frac{1}{2}\left(\lambda_{5}^{\prime}-\lambda_{4}^{\prime}\right) v^{2},
\end{aligned}
$$

which corresponds to a stable minimum if $0<\lambda_{5}^{\prime}<\lambda^{\prime}$ and $\lambda_{4}^{\prime}<\lambda_{5}^{\prime}$. The mass degeneracy of $H$ and $A$ is again a result of a residual $\mathrm{U}(1)^{\prime}$ symmetry that is preserved by the vacuum.

Third, if $\lambda_{5}^{\prime}=0$, then the GCP3 symmetry of the scalar potential is promoted to an $\mathrm{SO}(3)$ symmetry. In this case,

$$
m_{h}^{2}=\lambda^{\prime} v^{2}, \quad m_{H}^{2}=m_{A}^{2}=0, \quad m_{H^{ \pm}}^{2}=-\frac{1}{2} \lambda_{4}^{\prime} v^{2},
$$

corresponding to a stable minimum if $\lambda_{4}^{\prime}<0$. In particular, the $\mathrm{SO}(3)$ symmetry is spontaneously broken down to $\mathrm{U}(1)$, which yields two massless scalars $H$ and $A$.

If only one of the two vevs is nonzero (i.e., $s_{2 \beta}=0$ ), then Eqs. (2.12) and (2.13) yield $m_{12}^{\prime 2}=0$. Setting $R=1$ and $Y_{1}=Y_{2}=-\frac{1}{2} \lambda^{\prime} v^{2}$ in Eqs. (5.26)-(5.29), we end up with

$$
\begin{aligned}
& m_{h}^{2}=\lambda^{\prime} v^{2}, \quad m_{H}^{2}=-\lambda_{5}^{\prime} v^{2}, \\
& m_{A}^{2}=0, \quad m_{H^{ \pm}}^{2}=-\frac{1}{2}\left(\lambda_{4}^{\prime}+\lambda_{5}^{\prime}\right) v^{2},
\end{aligned}
$$

which coincides with the mass spectrum given in Eq. (7.35). If in addition we set $\lambda_{5}^{\prime}=0$, then we obtain the mass spectrum of Eq. (7.37).

\section{The landscape of ERPS4-Parts II(b) and II(c): Scalar potential with a softly broken or unbroken GCP3 or SO(3) symmetry}

Tables IX and X provide summaries of the landscape of possible scalar potentials in the subspace of the ERPS4 regime where the GCP3 or $\mathrm{SO}(3)$ symmetry of the scalar potential is either softly broken $\left(m_{11}^{\prime 2} \neq m_{22}^{\prime 2}\right.$ and/or $\left.m_{12}^{\prime 2} \neq 0\right)$ or unbroken $\left(m_{11}^{\prime 2}=m_{22}^{\prime 2}\right.$ and $\left.m_{12}^{\prime 2}=0\right)$.

Using the results of Sec. VIII, one can check that each entry of Tables IX and X can be matched up with a corresponding entry of Table VIII (and vice versa).

The analysis presented in this section can be repeated for the closely related $\mathrm{GCP}^{\prime}$ basis, where $\lambda_{1}^{\prime}=\lambda_{2}^{\prime}=\lambda_{3}^{\prime}+\lambda_{4}^{\prime}-\lambda_{5}^{\prime}$ and $\operatorname{Im} \lambda_{5}^{\prime}=\lambda_{6}^{\prime}=\lambda_{7}^{\prime}=0$. Details are left for the reader.

\section{TRANSFORMING BETWEEN THE $\mathrm{U}(1) \otimes \Pi_{2}$ BASIS AND GCP3 BASIS}

In Ref. [41], it was shown that the $\mathrm{U}(1) \otimes \Pi_{2}$ and GCP3-symmetric scalar potentials are in fact the same scalar potential expressed in different scalar field bases.

TABLE IX. Landscape of the ERPS4-Part II(b): Scalar potentials of the 2HDM with either an unbroken or softly broken GCP3 symmetry that is manifestly realized in the $\Phi$-basis. In all cases, $\lambda \equiv \lambda_{1}^{\prime}=\lambda_{2}^{\prime}=\lambda_{3}^{\prime}+\lambda_{4}^{\prime}+\lambda_{5}^{\prime}$, (with $\lambda_{5}^{\prime}$ real and nonzero) and $\lambda_{6}^{\prime}=\lambda_{7}^{\prime}=0$, and $C P$ is conserved by the scalar potential and vacuum. An exact Higgs alignment in the ERPS4 is realized in the inert limit where $Y_{3}=Z_{6}=Z_{7}=0$.

\begin{tabular}{lccccc}
\hline \hline$\beta^{\prime}$ & $\xi^{\prime}$ & $m_{11}^{\prime 2}, m_{22}^{\prime 2}$ & $m_{12}^{\prime 2} e^{i \xi^{\prime}}$ & Higgs alignment & Comment \\
\hline$s_{2 \beta^{\prime}} c_{2 \beta^{\prime}} \neq 0$ & $\sin 2 \xi^{\prime} \neq 0$ & $m_{11}^{\prime 2} \neq m_{22}^{\prime 2}$ & Complex & No & \\
$s_{2 \beta^{\prime}} c_{2 \beta^{\prime}} \neq 0$ & $\cos \xi^{\prime}=0$ & $m_{11}^{\prime 2} \neq m_{22}^{\prime 2}$ & Real & No & \\
$c_{2 \beta^{\prime}}=0$ & $\sin 2 \xi^{\prime} \neq 0$ & $m_{11}^{\prime 2}=m_{22}^{\prime 2}$ & Real $(\neq 0)$ & No & \\
$c_{2 \beta^{\prime}}=0$ & $\sin \xi^{\prime}=0$ & $m_{11}^{\prime 2}=m_{22}^{\prime 2}$ & Real $(\neq 0)$ & Yes & \\
$c_{2 \beta^{\prime}}=0$ & $\cos \xi^{\prime}=0$ & $m_{11}^{\prime 2}=m_{22}^{\prime 2}$ & Real $\neq 0)$ & Yes & $m_{H}^{2}=m_{A}^{2}>0$ \\
$s_{2 \beta^{\prime}} c_{2 \beta^{\prime}} \neq 0$ & $\sin \xi^{\prime}=0$ & $m_{11}^{\prime 2} \neq m_{22}^{\prime 2}$ & Real $(\neq 0)$ & Yes & \\
$s_{2 \beta^{\prime}}=0$ & & $m_{11}^{\prime 2} \neq m_{22}^{\prime 2}$ & 0 & Yes & \\
$s_{2 \beta^{\prime}} \neq 0$ & $\sin \xi^{\prime}=0$ & $m_{11}^{\prime 2}=m_{22}^{\prime 2}$ & 0 & Yes & One massless scalar \\
$c_{2 \beta^{\prime}}=0$ & $\cos \xi^{\prime}=0$ & $m_{11}^{\prime 2}=m_{22}^{\prime 2}$ & 0 & Yes & $m_{H}^{2}=m_{A}^{2}>0$ \\
$s_{2 \beta^{\prime}}=0$ & & $m_{11}^{\prime 2}=m_{22}^{\prime 2}$ & 0 & Yes & One massless scalar \\
\hline \hline
\end{tabular}

TABLE X. Landscape of the ERPS4-Part II(c): Scalar potentials of the 2HDM with either an unbroken or softly broken SO(3) symmetry that is manifestly realized in the $\Phi$-basis. In all cases, $\lambda \equiv \lambda_{1}^{\prime}=\lambda_{2}^{\prime}=\lambda_{3}^{\prime}+\lambda_{4}^{\prime}$ and $\lambda_{5}^{\prime}=\lambda_{6}^{\prime}=\lambda_{7}^{\prime}=0$, and $C P$ is conserved by the scalar potential and vacuum. In all cases of an unbroken or softly broken $\mathrm{SO}(3)$ symmetric scalar potential, an exact Higgs alignment is realized as a consequence of $Y_{3}=Z_{6}=Z_{7}=0$.

\begin{tabular}{lcccc}
\hline \hline$\beta^{\prime}$ & $m_{11}^{\prime 2}, m_{22}^{\prime 2}$ & $m_{12}^{\prime 2} e^{i \xi^{\prime}}$ & Higgs alignment & Comment \\
\hline$s_{2 \beta^{\prime}} c_{2 \beta^{\prime}} \neq 0$ & $m_{11}^{\prime 2} \neq m_{22}^{\prime 2}$ & Real $(\neq 0)$ & Yes & $m_{H}^{2}=m_{A}^{2}>0$ \\
$c_{2 \beta^{\prime}}=0$ & $m_{11}^{\prime 2}=m_{22}^{\prime 2}$ & Real $(\neq 0)$ & Yes & $m_{H}^{2}=m_{A}^{2}>0$ \\
$s_{2 \beta^{\prime}}=0$ & $m_{11}^{\prime 2} \neq m_{22}^{\prime 2}$ & 0 & Yes & $m_{H}^{2}=m_{A}^{2}>0$ \\
& $m_{11}^{\prime 2}=m_{22}^{\prime 2}$ & 0 & Yes & $m_{H}^{2}=m_{A}^{2}=0$ \\
\hline \hline
\end{tabular}


In this section, we extend this result to the softly broken $\mathrm{U}(1) \otimes \Pi_{2}$ and GCP3-symmetric scalar potentials by providing an explicit mapping between the corresponding scalar potential parameters.

Consider the following unitary transformation:

$$
U=\frac{e^{i \phi}}{\sqrt{2}}\left(\begin{array}{cc}
1 & -i \\
-i & 1
\end{array}\right),
$$

where the phase $\phi$ is determined in Eq. (8.13). Starting from the $\mathrm{U}(1) \otimes \Pi_{2}$ basis defined in Sec. VI, it then follows that (independently of the choice of $\phi$ )

$$
\begin{gathered}
\lambda^{\prime}=\lambda_{1}^{\prime}=\lambda_{2}^{\prime}=\frac{1}{2} \lambda(1+R), \\
\lambda_{3}^{\prime}=\lambda_{3}+\frac{1}{2} \lambda(1-R), \\
\lambda_{4}^{\prime}=\lambda_{4}+\frac{1}{2} \lambda(1-R), \\
\lambda_{5}^{\prime}=-\frac{1}{2} \lambda(1-R), \\
\lambda_{6}^{\prime}=-\lambda_{7}^{\prime}=0,
\end{gathered}
$$

where $R \equiv\left(\lambda_{3}+\lambda_{4}\right) / \lambda$. In particular, $\lambda_{5}^{\prime}=\lambda^{\prime}-\lambda_{3}^{\prime}-\lambda_{4}^{\prime}$ is real and $\lambda_{6}^{\prime}=\lambda_{7}^{\prime}=0$, corresponding to the GCP3 basis defined in Sec. VII. In addition, the corresponding softbreaking squared mass parameters are

$$
\begin{aligned}
& m_{11}^{\prime 2}=\frac{1}{2}\left(m_{11}^{2}+m_{22}^{2}\right)+\operatorname{Im} m_{12}^{2}, \\
& m_{22}^{\prime 2}=\frac{1}{2}\left(m_{11}^{2}+m_{22}^{2}\right)-\operatorname{Im} m_{12}^{2}, \\
& m_{12}^{\prime 2}=\operatorname{Re} m_{12}^{2}+\frac{1}{2} i\left(m_{22}^{2}-m_{11}^{2}\right) .
\end{aligned}
$$

Finally, the vevs in the GCP3 basis are given by

$$
\begin{aligned}
v_{1}^{\prime} & =\frac{e^{i \phi}}{\sqrt{2}}\left(v_{1}-i v_{2} e^{i \xi}\right), \\
v_{2}^{\prime} e^{i \xi^{\prime}} & =-e^{i \phi} \frac{i}{\sqrt{2}}\left(v_{1}+i v_{2} e^{i \xi}\right),
\end{aligned}
$$

where $v_{1}^{\prime} \equiv v c_{\beta^{\prime}}$ and $v_{2}^{\prime} \equiv v s_{\beta^{\prime}}$ are real and positive. Hence,

$$
\begin{aligned}
& c_{\beta^{\prime}}=\frac{1}{\sqrt{2}}\left(1+s_{2 \beta} \sin \xi\right)^{1 / 2}, \\
& s_{\beta^{\prime}}=\frac{1}{\sqrt{2}}\left(1-s_{2 \beta} \sin \xi\right)^{1 / 2},
\end{aligned}
$$

and it immediately follows that

$$
s_{2 \beta^{\prime}}^{2}=1-s_{2 \beta}^{2} \sin ^{2} \xi .
$$

By convention, $0 \leq \beta^{\prime} \leq \frac{1}{2} \pi$ (or equivalently, $\sin 2 \beta^{\prime} \geq 0$ ).

The phase $\phi$ is determined by the positivity of $v_{1}^{\prime}$. Hence, it follows that

$$
e^{i \phi}=\frac{c_{\beta}+i s_{\beta} e^{-i \xi}}{\left(1+s_{2 \beta} \sin \xi\right)^{1 / 2}} .
$$

Then, Eq. (8.10) yields

$$
e^{i \xi^{\prime}} s_{\beta^{\prime}}=-\frac{i}{\sqrt{2}} \frac{c_{2 \beta}+i s_{2 \beta} \cos \xi}{\left(1+s_{2 \beta} \sin \xi\right)^{1 / 2}} .
$$

Likewise, the relative phase $\xi^{\prime}$ is given by

$$
e^{i \xi^{\prime}}=\frac{s_{2 \beta} \cos \xi-i c_{2 \beta}}{\left(1-s_{2 \beta}^{2} \sin ^{2} \xi\right)^{1 / 2}}
$$

That is,

$$
\begin{aligned}
\sin \xi^{\prime} & =\frac{-c_{2 \beta}}{\left(1-s_{2 \beta}^{2} \sin ^{2} \xi\right)^{1 / 2}}, \\
\cos \xi^{\prime} & =\frac{s_{2 \beta} \cos \xi}{\left(1-s_{2 \beta}^{2} \sin ^{2} \xi\right)^{1 / 2}} .
\end{aligned}
$$

Consequently, Eqs. (8.12) and (8.16) yield

$$
s_{2 \beta^{\prime}} \sin \xi^{\prime}=-c_{2 \beta} .
$$

Finally, if $\beta=\frac{1}{4} \pi$ and $\sin \xi= \pm 1$, then one of the vevs vanishes. It then follows that $s_{2 \beta^{\prime}}=0$, in which case $\xi^{\prime}$ is indeterminate if $s_{\beta^{\prime}}=0$ and $\xi^{\prime}=0$ if $c_{\beta^{\prime}}=0$.

Using Eqs. (8.9), (8.12) and (8.15), it is instructive to note that

$\frac{2 \operatorname{Re}\left(m_{12}^{\prime 2} e^{i \xi^{\prime}}\right)}{s_{2 \beta^{\prime}}}=\frac{2 \operatorname{Re}\left(m_{12}^{2}\right) s_{2 \beta} \cos \xi+c_{2 \beta}\left(m_{22}^{2}-m_{11}^{2}\right)}{1-s_{2 \beta}^{2} \sin ^{2} \xi}$.

In light of Eq. (6.4), it follows that

$$
\begin{aligned}
\operatorname{Re} m_{12}^{2} & =\operatorname{Re}\left(m_{12}^{2} e^{i \xi}\right) \cos \xi+\operatorname{Im}\left(m_{12}^{2} e^{i \xi}\right) \sin \xi \\
& =\operatorname{Re}\left(m_{12}^{2} e^{i \xi}\right) \cos \xi
\end{aligned}
$$

Hence, after using Eqs. (6.2) and (6.3) for $m_{22}^{2}-m_{11}^{2}$ and Eq. (8.19) for $\mathrm{Re} m_{12}^{2}$, it then follows that Eq. (8.18) yields

$\frac{2 \operatorname{Re}\left(m_{12}^{\prime 2} e^{i \xi^{\prime}}\right)}{s_{2 \beta^{\prime}}}=\frac{2 \operatorname{Re}\left(m_{12}^{2} e^{i \xi}\right)}{s_{2 \beta}}+\frac{\lambda v^{2}(1-R) c_{2 \beta}^{2}}{2\left(1-s_{2 \beta}^{2} \sin ^{2} \xi\right)}$.

In particular, Eq. (7.20) yields 


$$
m_{A}^{2}=\frac{2 \operatorname{Re}\left(m_{12}^{\prime 2} e^{i \xi^{\prime}}\right)}{s_{2 \beta^{\prime}}}+\lambda_{5}^{\prime} v^{2} \sin ^{2} \xi^{\prime}=\frac{2 \operatorname{Re}\left(m_{12}^{2} e^{i \xi}\right)}{s_{2 \beta}},
$$

after employing Eqs. (8.5) and (8.16). Comparing with Eq. (6.14), we see that one obtains the same result for $m_{A}^{2}$ in the GCP3 basis and the $\mathrm{U}(1) \otimes \Pi_{2}$ basis respectively, as required. Note that the same conclusion can be drawn by plugging the results of Eqs. (8.2), (8.7) and (8.8) into Eq. (7.21), which reproduces the result of Eq. (6.15).

For completeness, we check that the scalar mass spectrum derived from the softly broken $\mathrm{U}(1) \otimes \Pi_{2}$ and GCP3symmetric scalar potentials coincide, as required. Plugging in the results of Eqs. (8.4) and (8.5) into Eq. (7.22) reproduces the result of Eq. (6.16) for $m_{H^{ \pm}}^{2}$. To check the squared masses of the neutral $C P$-even scalars, we plug the results of Eqs. (8.2) and (8.5) into Eq. (7.27), which reproduces the result of Eq. (6.21). Finally, we plug in the results of Eqs. (8.2), (8.5) and (8.17) into Eq. (7.28), which reproduces the result of Eq. (6.22).

As a final check of our computations, one can verify that the invariant quantities, $Y_{2}, Z_{1}, \ldots, Z_{4},\left|Z_{5}\right|,\left|Z_{6}\right|$ and $Z_{5}^{*} Z_{6}^{2}$ are independent of the choice of basis. For example, starting from the GCP3 basis,

$$
\begin{aligned}
Z_{5}^{*} Z_{6}^{2} & =-\lambda_{5}^{\prime} s_{2 \beta^{\prime}}^{2} \sin ^{2} \xi^{\prime}\left|Z_{5}\right|^{2} \\
& =-\lambda_{5}^{\prime 3} s_{2 \beta^{\prime}}^{2} \sin ^{2} \xi^{\prime}\left(1-s_{2 \beta^{\prime}}^{2} \sin ^{2} \xi^{\prime}\right)^{2} \\
& =\frac{1}{8} \lambda^{3}(1-R)^{3} c_{2 \beta}^{2} s_{2 \beta}^{4},
\end{aligned}
$$

in agreement with Eqs. (6.10) and (6.11). One can also check that all the other invariants yield the same values in the GCP3 and $\mathrm{U}(1) \otimes \Pi_{2}$ bases.

Given a softly broken $\mathrm{U}(1) \otimes \Pi_{2}$-symmetric scalar potential that is displayed in the $\mathrm{U}(1) \otimes \Pi_{2}$ basis where the softly broken symmetry is manifestly realized, it may turn out that the scalar potential is invariant under some discrete or continuous subgroup of $\mathrm{U}(1) \otimes \Pi_{2}$. It is of interest to determine implications of this invariance for the scalar potential parameters when expressed in the GCP3 basis. We proceed by assuming that the scalar potential, which is specified in Eq. (C1) in terms of squared mass parameters $Y_{a \bar{b}}$ and dimensionless parameters $Z_{a b, c d}$ [defined in Eq. (C4)], is invariant under the transformation,

$$
\Phi_{a} \rightarrow X_{a \bar{b}} \Phi_{b}, \quad \Phi_{\bar{a}}^{\dagger} \rightarrow \Phi_{\bar{b}}^{\dagger} X_{b \bar{a}}^{\dagger} .
$$

That is [cf. Eqs. (C7) and (C8)],

$$
Y=X Y X^{\dagger}, \quad Z=(X \otimes X) Z\left(X^{\dagger} \otimes X^{\dagger}\right) .
$$

Consider a change of scalar field basis specified by $U$ [e.g., $U$ specified in Eq. (8.1) transforms the $\mathrm{U}(1) \otimes \Pi_{2}$ basis into the GCP3 basis]. In light of Eqs. (C7) and (C8), $Y^{\prime}=U Y U^{\dagger}$ yields the squared mass parameters in the new basis, and $Z^{\prime}=(U \otimes U) Z\left(U^{\dagger} \otimes U^{\dagger}\right)$ yields the dimensionless parameters in the new basis. In the new basis, the symmetry transformation matrix $X$ will be denoted by $X^{\prime}$. That is, $Y^{\prime}=X^{\prime} Y^{\prime} X^{\prime \dagger}$, since the parameters in the new basis are invariant with respect to the transformations induced by $X^{\prime}$. It then follows that $U Y U^{\dagger}=X^{\prime}\left(U Y U^{\dagger}\right) X^{\prime \dagger}$, which yields

$$
Y=\left(U^{\dagger} X^{\prime} U\right) Y\left(U^{\dagger} X^{\prime} U\right)^{\dagger} .
$$

Comparing this result with Eq. (8.24), we can conclude that

$$
X^{\prime}=e^{i \zeta} U X U^{\dagger},
$$

where the complex phase factor $e^{i \zeta}$ is arbitrary and can be chosen for convenience as it corresponds to an additional hypercharge $\mathrm{U}(1)_{\mathrm{Y}}$ transformation, which has no effect on the scalar potential parameters. One can now check that $Z^{\prime}=\left(X^{\prime} \otimes X^{\prime}\right) Z^{\prime}\left(X^{\prime \dagger} \otimes X^{\prime \dagger}\right)$ by inserting Eq. (8.26) for $X^{\prime}$ and evaluating the products and Hermitian conjugates according to Eqs. (C10) and (C11).

We shall now employ Eq. (8.26) in several examples. First, suppose that the softly broken $\mathrm{U}(1) \otimes \Pi_{2}$-symmetric scalar potential is invariant with respect to $\mathbb{Z}_{2}$. Using Table I, it follows that $X=\left(\begin{array}{cc}1 & 0 \\ 0 & -1\end{array}\right)$. Hence, when transformed to the GCP3 basis using $U$ specified in Eq. (8.1), and choosing $e^{i \zeta}=-i$, it follows that

$$
X^{\prime}=\left(\begin{array}{cc}
0 & 1 \\
-1 & 0
\end{array}\right)
$$

which corresponds to the $\Pi_{2}^{\prime}$ symmetry defined in Table IV. This is easily checked using Eqs. (8.7)-(8.9). In particular, if $\operatorname{Im} m_{12}^{2}=0$ and $m_{11}^{2} \neq m_{22}^{2}$, then it follows that $m_{11}^{\prime 2}=$ $m_{22}^{\prime 2}$ and $\mathrm{Re} m_{12}^{\prime 2}=0$ (cf. Table VI).

Second, suppose that the softly broken $\mathrm{U}(1) \otimes \Pi_{2^{-}}$ symmetric scalar potential is invariant with respect to $\Pi_{2}$. Using Table I, it follows that $X=\left(\begin{array}{ll}0 & 1 \\ 1 & 0\end{array}\right)$. Hence, when transformed to the GCP3 basis using $U$ specified in Eq. (8.1), and choosing $e^{i \zeta}=1$, it follows that $X^{\prime}=X$. That is, the scalar potential in the GCP3 basis also exhibits a $\Pi_{2}$ symmetry. This is easily checked using Eqs. (8.7)-(8.9). Namely, if $m_{11}^{2}=m_{22}^{2}$ and $\operatorname{Im} m_{12}^{2}=0$ (cf. Table III), then the same relations also hold for the primed parameters.

Third, suppose that the softly broken $U(1) \otimes \Pi_{2}-$ symmetric scalar potential is invariant with respect to $\mathrm{U}(1)$. Using Table I, it follows that $X=\left(\begin{array}{cc}e^{-i \theta} & 0 \\ 0 & e^{i \theta}\end{array}\right)$, where $-\frac{1}{2} \pi<\theta \leq \frac{1}{2} \pi$. Transforming to the GCP3 basis using $U$ given in Eq. (8.1), and choosing $e^{i \zeta}=1$, we obtain

$X^{\prime}=\left(\begin{array}{cc}\cos \theta & \sin \theta \\ -\sin \theta & \cos \theta\end{array}\right), \quad$ for $-\frac{1}{2} \pi<\theta \leq \frac{1}{2} \pi$, 
which defines the $\mathrm{U}(1)^{\prime}$ symmetry transformation. In particular, if $\lambda_{1}=\lambda_{2}$ and $m_{12}^{2}=\lambda_{5}=\lambda_{6}=\lambda_{7}=0$ then Eqs. (8.2)-(8.9) yield, $m_{11}^{\prime 2}=m_{22}^{\prime 2}, \quad \operatorname{Re} m_{12}^{\prime 2}=0, \quad \lambda_{5}^{\prime}=$ $\lambda^{\prime}-\lambda_{3}^{\prime}-\lambda_{4}^{\prime}$ is real and $\lambda_{6}^{\prime}=\lambda_{7}^{\prime}=0$, which are the constraints due to $\mathrm{U}(1)^{\prime}$ as indicated in Table VI.

As our final example, we reconsider the residual unbroken symmetry in the case of a softly broken $U(1) \otimes \Pi_{2}-$ symmetric scalar potential when $c_{2 \beta}=0$. Below Eq. (6.26), we noted that in this limiting case, after performing a rephasing to set $\xi=0$, the residual symmetry of the scalar potential and vacuum was $\Pi_{2}$. However, in this example, we shall keep $\xi$ arbitrary. In order to accommodate $\xi \neq 0$ we define a new discrete symmetry,

$$
\Pi_{2}^{(\alpha)}: \Phi_{1} \rightarrow e^{-i \alpha} \Phi_{2}, \quad \Phi_{2} \rightarrow e^{i \alpha} \Phi_{1},
$$$$
\text { where } \alpha \text { is a fixed real parameter. }
$$

Note that when $\Pi_{2}^{(\alpha)}$ is applied twice, one obtains the identity. This means that for any fixed value of $\alpha$, the $\Pi_{2}^{(\alpha)}$ symmetry is equivalent to a $\mathbb{Z}_{2}$ symmetry that is manifestly realized in a different scalar field basis.

Of course, for $\alpha=0$, we regain the $\Pi_{2}$ symmetry. Moreover, up to an overall hypercharge $\mathrm{U}(1)_{\mathrm{Y}}$ transformation, the $\Pi_{2}^{\prime}$ symmetry corresponds to $\alpha=\frac{1}{2} \pi$. If the scalar potential in the $\Phi$-basis is invariant under $\Pi_{2}^{(\alpha)}$, then it follows that

$$
\begin{aligned}
m_{11}^{2} & =m_{22}^{2}, \quad \operatorname{Im}\left(m_{12}^{2} e^{i \alpha}\right)=0, \quad \lambda_{1}=\lambda_{2}, \\
\operatorname{Im}\left(\lambda_{5} e^{2 i \alpha}\right) & =0, \quad \lambda_{7}=\lambda_{6}^{*} e^{-2 i \alpha} .
\end{aligned}
$$

In light of Eq. (6.4), it follows that for $c_{2 \beta}=0$, the residual symmetry of the softly broken $\mathrm{U}(1) \otimes \Pi_{2}$-symmetric scalar potential and vacuum is $\Pi_{2}^{(\xi)}$ (for any fixed value of $\xi$ ).

Suppose that the $\Pi_{2}^{(\alpha)}$ symmetry is unbroken by the scalar potential in the $\mathrm{U}(1) \otimes \Pi_{2}$ basis. Then, we can deduce the corresponding symmetry in the GCP3 basis. In this example, $X=\left(\begin{array}{cc}0 & e^{i \alpha} \\ e^{-i \alpha} & 0\end{array}\right)$, where $\alpha$ is a fixed real parameter. Hence, when transformed to the GCP3 basis using $U$ specified in Eq. (8.1), and choosing $e^{i \zeta}=1$, it follows that $X^{\prime}=\left(\begin{array}{cc}\sin \alpha & \cos \alpha \\ \cos \alpha & -\sin \alpha\end{array}\right), \quad$ for a fixed real value of $\alpha$.

Without loss of generality, we may take $-\frac{1}{2} \pi<\alpha \leq \frac{1}{2} \pi$ [since $\alpha \rightarrow \alpha+\pi$ yields a hypercharge $\mathrm{U}(1)_{\mathrm{Y}}$ transformation]. We shall denote this symmetry by

$$
\begin{aligned}
\bar{\Pi}_{2}^{(\alpha)}: \Phi_{1} & \rightarrow \Phi_{1} \sin \alpha+\Phi_{2} \cos \alpha \\
\Phi_{2} & \rightarrow \Phi_{1} \cos \alpha-\Phi_{2} \sin \alpha .
\end{aligned}
$$

Note that for $\alpha=0\left[\alpha=\frac{1}{2} \pi\right]$, the $\bar{\Pi}_{2}^{(\alpha)}$ symmetry coincides with $\Pi_{2}\left[\mathbb{Z}_{2}\right]$. That is, $\bar{\Pi}_{2}^{(\alpha)}$ provides an interpolation from the $\Pi_{2}$ to the $\mathbb{Z}_{2}$ symmetry.

Imposing the $\bar{\Pi}_{2}^{(\alpha)}$ symmetry on the parameters of the scalar potential in the $\Phi$-basis for a fixed value of $\alpha \neq 0, \frac{1}{2} \pi$, it follows that

$$
\begin{gathered}
\operatorname{Im} m_{12}^{2}=\operatorname{Im} \lambda_{5}=\operatorname{Im} \lambda_{6}=\operatorname{Im} \lambda_{7}=0, \\
m_{22}^{2}-m_{11}^{2}=2 \tan \alpha \operatorname{Re} m_{12}^{2}, \\
\lambda_{1}-\lambda_{2}=2 \tan \alpha \operatorname{Re}\left(\lambda_{6}+\lambda_{7}\right), \\
\lambda_{1}+\lambda_{2}-2\left(\lambda_{3}+\lambda_{4}+\operatorname{Re} \lambda_{5}\right)=4 \cot 2 \alpha \operatorname{Re}\left(\lambda_{6}-\lambda_{7}\right) .
\end{gathered}
$$

Applying the above results to the softly broken GCP3-symmetric scalar potential, we set $\lambda^{\prime} \equiv \lambda_{1}^{\prime}=\lambda_{2}^{\prime}=$ $\lambda_{3}^{\prime}+\lambda_{4}^{\prime}+\lambda_{5}^{\prime}$ and $\operatorname{Im} \lambda_{5}^{\prime}=\lambda_{6}^{\prime}=\lambda_{7}^{\prime}=0$. Note that Eqs. (8.33)-(8.36) are consistent with these constraints. In addition, the softly broken GCP3-symmetric scalar potential preserves the $\bar{\Pi}_{2}^{(\alpha)}$ symmetry in two cases: (i) if $m_{12}^{\prime 2}$ is real and nonzero then $m_{11}^{\prime 2} \neq m_{22}^{\prime 2}$ [in which case, $\alpha$ is determined from Eq. (8.34)]; or (ii) if $m_{12}^{\prime 2}=0$ then $m_{11}^{\prime 2}=m_{22}^{\prime 2}$ (in which case $\bar{\Pi}_{2}^{(\alpha)}$, which is a symmetry of the scalar potential for all values of $\alpha$, is promoted to an unbroken GCP3 symmetry).

For example, in the inert limit of the softly broken GCP3-symmetric scalar potential, where $s_{2 \beta^{\prime}} c_{2 \beta^{\prime}} \neq 0$, $\sin \xi^{\prime}=0, m_{11}^{\prime 2} \neq m_{22}^{\prime 2}$ and $m_{12}^{\prime 2} e^{i \xi^{\prime}}$ is real [See Table IX], we see that the conditions for the $\bar{\Pi}_{2}^{(\alpha)}$ symmetry are satisfied. Moreover, one can show that the $\bar{\Pi}_{2}^{(\alpha)}$ symmetry is unbroken by the vacuum as follows. Using Eqs. (7.2)-(7.4) under the assumption that $\sin \xi^{\prime}=0$, it follows that $m_{12}^{\prime 2} e^{i \xi^{\prime}}=m_{12}^{\prime 2} \cos \xi^{\prime}= \pm m_{12}^{\prime 2}$ is real (where \pm corresponds to $\xi^{\prime}=0$ or $\xi^{\prime}=\pi$, respectively), and

$$
m_{22}^{\prime 2}-m_{11}^{\prime 2}= \pm \frac{2 m_{12}^{\prime 2} c_{2 \beta^{\prime}}}{s_{2 \beta^{\prime}}}
$$

Hence, Eq. (8.34) yields $\tan \alpha= \pm \cot 2 \beta^{\prime}$. In the convention where $-\frac{1}{2} \pi<\alpha \leq \frac{1}{2} \pi$ and $0 \leq \beta^{\prime} \leq \frac{1}{2} \pi$, it follows that $\sin \alpha= \pm \cos 2 \beta^{\prime}$ and $\cos \alpha=\sin 2 \beta^{\prime}$, or equivalently

$$
\alpha= \pm\left(\frac{1}{2} \pi-2 \beta^{\prime}\right) .
$$

The $\bar{\Pi}_{2}^{(\alpha)}$ symmetry is unbroken by the vacuum if

$$
X^{\prime}\left(\begin{array}{c}
v_{1}^{\prime} \\
v_{2}^{\prime} e^{i \xi^{\prime}}
\end{array}\right)= \pm\left(\begin{array}{c}
v_{1}^{\prime} \\
v_{2}^{\prime} e^{i \xi^{\prime}}
\end{array}\right)
$$


where the \pm sign reflects the fact that $\left\{\left\langle\Phi_{1}^{0}\right\rangle,\left\langle\Phi_{2}^{0}\right\rangle\right\}$ is equivalent to $\left\{-\left\langle\Phi_{1}^{0}\right\rangle,-\left\langle\Phi_{2}^{0}\right\rangle\right\}$, as the two are related by a hypercharge $\mathrm{U}(1)_{\mathrm{Y}}$ transformation. After using the value of $\alpha$ obtained in Eq. (8.38) to determine $X^{\prime}$ [cf. Eq. (8.31)], it follows that the $\bar{\Pi}_{2}^{(\alpha)}$ symmetry is unbroken by the vacuum since the following equation is an identity:

$$
\left(\begin{array}{cc} 
\pm c_{2 \beta^{\prime}} & s_{2 \beta^{\prime}} \\
s_{2 \beta^{\prime}} & \mp c_{2 \beta^{\prime}}
\end{array}\right)\left(\begin{array}{c}
c_{\beta^{\prime}} \\
\pm s_{\beta^{\prime}}
\end{array}\right)= \pm\left(\begin{array}{c}
c_{\beta^{\prime}} \\
\pm s_{\beta^{\prime}}
\end{array}\right) .
$$

Consequently, one can conclude that the $\bar{\Pi}_{2}^{(\alpha)}$ symmetry is responsible for the inert limit (and the attendant exact Higgs alignment) in this case.

In some applications, it is useful to invert the relations obtained in Eqs. (8.2)-(8.9). This can be achieved by starting from the GCP3 basis and employing the unitary transformation

$$
U^{-1}=\frac{e^{-i \phi}}{\sqrt{2}}\left(\begin{array}{ll}
1 & i \\
i & 1
\end{array}\right)
$$

The resulting $\mathrm{U}(1) \otimes \Pi_{2}$ basis parameters are

$$
\begin{gathered}
\lambda=\lambda^{\prime}-\lambda_{5}^{\prime}, \\
\lambda_{3}=\lambda_{3}^{\prime}+\lambda_{5}^{\prime}, \\
\lambda_{4}=\lambda_{4}^{\prime}+\lambda_{5}^{\prime}, \\
\lambda R=\lambda^{\prime}+\lambda_{5}^{\prime}, \\
\lambda_{5}=\lambda_{6}=\lambda_{7}=0 .
\end{gathered}
$$

In addition, the corresponding soft-breaking squared mass parameters are

$$
\begin{aligned}
& m_{11}^{2}=\frac{1}{2}\left(m_{11}^{\prime 2}+m_{22}^{\prime 2}\right)-\operatorname{Im} m_{12}^{\prime 2}, \\
& m_{22}^{2}=\frac{1}{2}\left(m_{11}^{\prime 2}+m_{22}^{\prime 2}\right)+\operatorname{Im} m_{12}^{\prime 2}, \\
& m_{12}^{2}=\operatorname{Re} m_{12}^{\prime 2}-\frac{1}{2} i\left(m_{22}^{\prime 2}-m_{11}^{\prime 2}\right) .
\end{aligned}
$$

Finally, the vevs in the $\mathrm{U}(1) \otimes \Pi_{2}$ basis are given by

$$
\begin{aligned}
v_{1} & =\frac{e^{-i \phi}}{\sqrt{2}}\left(v_{1}^{\prime}+i v_{2}^{\prime} e^{i \xi^{\prime}}\right), \\
v_{2} e^{i \xi} & =e^{-i \phi} \frac{i}{\sqrt{2}}\left(v_{1}^{\prime}-i v_{2}^{\prime} e^{i \xi^{\prime}}\right),
\end{aligned}
$$

where $v_{1}$ and $v_{2}$ are real and positive. Hence,

$$
\begin{aligned}
& c_{\beta}=\frac{1}{\sqrt{2}}\left(1-s_{2 \beta^{\prime}} \sin \xi^{\prime}\right)^{1 / 2}, \\
& s_{\beta}=\frac{1}{\sqrt{2}}\left(1+s_{2 \beta^{\prime}} \sin \xi^{\prime}\right)^{1 / 2},
\end{aligned}
$$

and it immediately follows that

$$
s_{2 \beta}^{2}=1-s_{2 \beta^{\prime}}^{2} \sin ^{2} \xi^{\prime} .
$$

By convention, $0 \leq \beta \leq \frac{1}{2} \pi$ (or equivalently, $\sin 2 \beta \geq 0$ ).

The phase $\phi$ is again fixed by the positivity of $v_{1}$, which yields

$$
e^{-i \phi}=\frac{c_{\beta^{\prime}}-i s_{\beta^{\prime}} e^{-i \xi^{\prime}}}{\left(1-s_{2 \beta^{\prime}} \sin \xi^{\prime}\right)^{1 / 2}},
$$

and is consistent with Eq. (8.13) after employing Eqs. (8.51) and (8.55). Then, Eq. (8.50) yields

$$
e^{i \xi} s_{\beta}=\frac{i}{\sqrt{2}} \frac{c_{2 \beta^{\prime}}-i s_{2 \beta^{\prime}} \cos \xi^{\prime}}{\left(1-s_{2 \beta^{\prime}} \sin \xi^{\prime}\right)^{1 / 2}}
$$

Likewise, $\xi$ is given by

$$
e^{i \xi}=\frac{s_{2 \beta^{\prime}} \cos \xi^{\prime}+i c_{2 \beta^{\prime}}}{\left(1-s_{2 \beta^{\prime}}^{2} \sin ^{2} \xi^{\prime}\right)^{1 / 2}}
$$

That is,

$$
\begin{aligned}
\sin \xi & =\frac{c_{2 \beta^{\prime}}}{\left(1-s_{2 \beta^{\prime}}^{2} \sin ^{2} \xi^{\prime}\right)^{1 / 2}}, \\
\cos \xi & =\frac{s_{2 \beta^{\prime}} \cos \xi^{\prime}}{\left(1-s_{2 \beta^{\prime}}^{2} \sin ^{2} \xi^{\prime}\right)^{1 / 2}} .
\end{aligned}
$$

Hence Eqs. (8.52) and (8.56) yield

$$
s_{2 \beta} \sin \xi=c_{2 \beta^{\prime}} .
$$

Once the $\mathrm{U}(1) \otimes \Pi_{2}$ basis parameters have been derived, one can perform one further rephasing to remove the phase $\xi$ (which is unphysical). Finally, if $\beta^{\prime}=\frac{1}{4} \pi$ and $\sin \xi^{\prime}= \pm 1$, then one of the vevs vanishes. It then follows that $s_{2 \beta}=0$, in which case $\xi$ is indeterminate if $s_{\beta}=0$ and $\xi=0$ if $c_{\beta}=0$.

The scalar masses obtained in the $\mathrm{U}(1) \otimes \Pi_{2}$ basis and the GCP3 basis were derived by applying Eq. (5.22). In employing this equation, a specific value of $\eta$ was chosen. The eigenvalues of $\mathcal{M}^{2}$ are independent of this choice. However, the identification of $H$ and $A$ depend on this choice in the inert limit. For a consistent treatment of the two basis choices, one should also transform $\eta$ when changing the scalar field basis according to Eq. (5.8). In particular, given the choice of $\eta=-\xi$ that was employed in 
the $\mathrm{U}(1) \otimes \Pi_{2}$ basis, the corresponding $\eta^{\prime}$ in the GCP3 basis is given by

$$
\begin{aligned}
e^{-i \eta^{\prime}} & =(\operatorname{det} U) e^{-i \eta}=e^{2 i \phi} e^{i \xi} \\
& =\frac{\left(c_{\beta^{\prime}}+i s_{\beta^{\prime}} e^{i \xi^{\prime}}\right)^{2}\left(s_{2 \beta^{\prime}} \cos \xi^{\prime}+i c_{2 \beta^{\prime}}\right)}{\left(1-s_{2 \beta^{\prime}} \sin \xi^{\prime}\right)\left(1-s_{2 \beta^{\prime}}^{2} \sin ^{2} \xi^{\prime}\right)^{1 / 2}},
\end{aligned}
$$

after employing Eq. (8.1) to obtain $\operatorname{det} U=e^{2 i \phi}$ and making use of Eqs. (8.53) and (8.55).

The numerator of Eq. (8.58) can be simplified with a little algebra,

$$
\begin{aligned}
\left(c_{\beta^{\prime}}\right. & \left.+i s_{\beta^{\prime}} e^{i \xi^{\prime}}\right)^{2}\left(s_{2 \beta^{\prime}} \cos \xi^{\prime}+i c_{2 \beta^{\prime}}\right) \\
& =e^{i \xi^{\prime}}\left[c_{2 \beta^{\prime}} \cos \xi^{\prime}+i\left(s_{2 \beta^{\prime}}-\sin \xi^{\prime}\right)\right]\left(s_{2 \beta^{\prime}} \cos \xi^{\prime}+i c_{2 \beta^{\prime}}\right) \\
& =e^{i \xi^{\prime}}\left(c_{2 \beta^{\prime}} \sin \xi^{\prime}+i \cos \xi^{\prime}\right)\left(1-s_{2 \beta^{\prime}} \sin \xi^{\prime}\right) . \\
& =i e^{i\left(\xi^{\prime}-\psi\right)}\left(1-s_{2 \beta^{\prime}} \sin \xi^{\prime}\right)\left(1-s_{2 \beta^{\prime}}^{2} \sin ^{2} \xi^{\prime}\right)^{1 / 2},
\end{aligned}
$$

where we have used Eq. (7.16) in the final step. Inserting this result back into Eq. (8.58) yields

$$
\eta^{\prime}=\psi-\xi^{\prime}-\frac{1}{2} \pi,
$$

which justifies the choice of $\eta$ that was employed in Eq. (7.17). Note that if $s_{2 \beta^{\prime}}=0$, then one of the two vevs in the GCP3 basis vanishes. If $c_{2 \beta^{\prime}}=1$, then Eq. (7.16) yields $\psi=\xi^{\prime}$ and we conclude that $\eta^{\prime}=-\frac{1}{2} \pi$. If $c_{2 \beta^{\prime}}=-1$, then $\psi=\xi^{\prime}=0$ (since in this case $\left\langle\Phi_{2}^{0}\right\rangle=v / \sqrt{2}$ is real and positive) and we again find that $\eta^{\prime}=-\frac{1}{2} \pi$. That is, $e^{-2 i \eta^{\prime}}=-1$, which is the motivation for the choice of $\eta$ employed in obtaining Eqs. (5.26)-(5.29). ${ }^{22}$

For a satisfying check of Eq. (8.60), one can compute the value of $Z_{5}$ in the GCP3 basis starting from its value in the $\mathrm{U}(1) \otimes \Pi_{2}$ basis given in Eq. (6.10). In performing this computation, one must remember to rephase $Z_{5}$ as indicated in Eq. (5.10). After making use of Eqs. (8.42), (8.45), (8.60) and (7.16), it then follows that

$$
\begin{aligned}
Z_{5} & =\frac{1}{2} \lambda s_{2 \beta}^{2}(1-R) e^{-2 i \xi} e^{-4 i \phi} \\
& =-\lambda_{5}^{\prime}\left(1-s_{2 \beta^{\prime}}^{2} \sin ^{2} \xi^{\prime}\right) e^{2 i \eta^{\prime}} \\
& =\lambda_{5}^{\prime} e^{-2 i \xi^{\prime}}\left(1-s_{2 \beta^{\prime}}^{2} \sin ^{2} \xi^{\prime}\right) e^{2 i \psi} \\
& =\lambda_{5}^{\prime} e^{-2 i \xi^{\prime}}\left(\cos \xi^{\prime}+i c_{2 \beta^{\prime}} \sin \xi^{\prime}\right)^{2},
\end{aligned}
$$

in agreement with Eq. (7.12).

Although cases in which one of the two vevs vanish appear to be isolated from the parameter regimes in which

\footnotetext{
${ }^{22}$ If $s_{2 \beta}=0$ in the $\mathrm{U}(1) \otimes \Pi_{2}$ basis then $Z_{5}=0$, in which case $m_{H}=m_{A}$ and the results of Eqs. (5.26)-(5.29) do not depend on the choice of $\eta$.
}

both vevs are nonvanishing, in fact the two parameter regimes can be regarded as being continuously connected. For example, starting from the GCP3 basis, the parameter regime in which one of the two vevs vanishes (i.e., $s_{2 \beta^{\prime}}=0$ ) implies that $m_{12}^{\prime 2}=0$ due to Eqs. (2.12) and (2.13). ${ }^{23}$ In light of Eq. (8.9), it follows that in terms of the $\mathrm{U}(1) \otimes \Pi_{2}$ basis parameters, $\operatorname{Re} m_{12}^{2}=0$ and $m_{11}^{2}=m_{22}^{2}$, but in general $\operatorname{Im} m_{12}^{2} \neq 0$. Moreover, Eq. (6.5) then yields $\beta=\frac{1}{4} \pi$. As expected, this parameter regime can be identified as the inert limit, independently of the basis choice. Nevertheless, it is clear that $\beta=\frac{1}{4} \pi$ is continuously connected to other regions of the parameter space in the $\mathrm{U}(1) \otimes \Pi_{2}$ basis. Similarly, the case of one vanishing vev in the $U(1) \otimes \Pi_{2}$ basis corresponds to $s_{2 \beta^{\prime}} \sin \xi= \pm 1$, which implies that $\beta^{\prime}=\frac{1}{4} \pi$ in the GCP3 basis. Finally, the inert limit in the $\mathrm{U}(1) \otimes \Pi_{2}$ basis when $R=1$ corresponds to the inert limit in the GCP3 basis when $\lambda_{5}^{\prime}=0$.

\section{THE HIGGS ALIGNMENT LIMIT}

The neutral scalar squared-mass matrix, $\mathcal{M}^{2}$, given in Eq. (5.22) is expressed with respect to a basis of neutral scalar interaction eigenstates, $\left\{\sqrt{2} \operatorname{Re} \mathcal{H}_{1}^{0}-v, \operatorname{Re} \mathcal{H}_{2}^{0}, \operatorname{Im} \mathcal{H}_{2}^{0}\right\}$, where $\mathcal{H}_{1}^{0}$ and $\mathcal{H}_{2}^{0}$ are the neutral components of the Higgs basis fields. The neutral scalar interaction eigenstate, $\varphi_{1} \equiv \sqrt{2} \operatorname{Re} \mathcal{H}_{1}^{0}-v$, possesses tree-level couplings to SM particles that coincide precisely with those of the SM Higgs boson. Consequently, if the mixing of $\varphi_{1}$ with $\operatorname{Re} \mathcal{H}_{2}^{0}$ and $\operatorname{Im} \mathcal{H}_{2}^{0}$ were to vanish exactly, then $\varphi_{1}$ would be a mass eigenstate with tree-level properties that are indistinguishable from those of the SM Higgs boson. In this case, the direction of $\varphi_{1}$ in field space is exactly aligned with the direction of the vacuum expectation value $v$. Hence, the limit of zero mixing described above is called the Higgs alignment limit [42-47].

In light of Eq. (5.22), it follows that the Higgs alignment is realized exactly if and only if $Z_{6}=0$, in which case we can identify $m_{\varphi_{1}}^{2}=Z_{1} v^{2}$. In this limit, the masses of the two other neutral scalars are not immediately constrained (beyond experimental bounds based on the absence of any newly discovered scalar states at the LHC). Although the observed Higgs boson at the LHC is SM-like, the precision of the current data allows for $10 \%-20 \%$ deviations from SM behavior of the Higgs boson couplings to vector bosons and third generation quarks and charged leptons [3-6]. Thus, the present Higgs data requires only an approximate Higgs alignment, which allows for a small mixing of $\varphi_{1}$ with the other two neutral scalar interaction eigenstates. This small mixing can be achieved in one

\footnotetext{
${ }^{23}$ Note that it follows that the ratio $\operatorname{Re}\left(m_{12}^{\prime 2} e^{i \xi^{\prime}}\right) / s_{2 \beta^{\prime}}$ that appears in Eq. (7.20) is indeterminate, in which case it can be replaced by $m_{A}^{2}-\lambda_{5}^{\prime} v^{2} \sin ^{2} \xi^{\prime}$, with $m_{A}^{2}$ being regarded as a free parameter.
} 
of two ways-either $\left|Z_{6}\right| \ll 1$ or $Y_{2} \gg v^{2}$. The latter corresponds to the decoupling limit of the 2HDM $[42,81]$.

For example, if $C P$ is conserved then the squared-mass matrix, $\mathcal{M}^{2}$, of the neutral scalars, expressed with respect to Higgs basis fields, breaks up into a $2 \times 2$ block and a $1 \times 1$ block,

$$
\mathcal{M}^{2}=\left(\begin{array}{ccc}
Z_{1} v^{2} & Z_{6} v^{2} & 0 \\
Z_{6} v^{2} & m_{A}^{2}+Z_{5} v^{2} & 0 \\
0 & 0 & m_{A}^{2}
\end{array}\right) .
$$

Once again, we see that $Z_{6}=0$ corresponds to exact Higgs alignment, whereas approximate Higgs alignment is realized when $\left|Z_{6}\right| \ll 1$ and/or $m_{A} \gg v$.

Diagonalizing the $2 \times 2$ block yields the $C P$-even Higgs mass eigenstates $H$ and $h$,

$$
\left(\begin{array}{c}
H \\
h
\end{array}\right)=\left(\begin{array}{cc}
c_{\beta-\alpha} & -s_{\beta-\alpha} \\
s_{\beta-\alpha} & c_{\beta-\alpha}
\end{array}\right)\left(\begin{array}{c}
\sqrt{2} \operatorname{Re} \mathcal{H}_{1}^{0}-v \\
\sqrt{2} \operatorname{Re} \mathcal{H}_{2}^{0}
\end{array}\right),
$$

where $m_{h} \leq m_{H}, c_{\beta-\alpha} \equiv \cos (\beta-\alpha)$ and $s_{\beta-\alpha} \equiv \sin (\beta-\alpha)$ in a convention where $0 \leq \beta-\alpha \leq \pi$. In a real $\Phi$-basis with real non-negative vevs, $\left\langle\Phi_{a}^{0}\right\rangle=v_{a} / \sqrt{2}(a=1,2), \tan \beta=$ $v_{2} / v_{1}$ and $\alpha$ is the mixing angle that diagonalizes the $C P$-even Higgs squared-mass matrix when expressed with respect to the $\left\{\sqrt{2} \operatorname{Re} \Phi_{1}^{0}-v_{1}, \sqrt{2} \operatorname{Re} \Phi_{2}^{0}-v_{2}\right\}$ basis. Nevertheless, the quantity $s_{\beta-\alpha}$ is independent of the choice of the scalar field basis.

After diagonalizing the matrix $\mathcal{M}_{H}^{2}$, the neutral $C P$-even scalar masses are given by

$$
\begin{aligned}
m_{H, h}^{2}= & \frac{1}{2}\left\{m_{A}^{2}+\left(Z_{1}+Z_{5}\right) v^{2}\right. \\
& \left. \pm \sqrt{\left[m_{A}^{2}-\left(Z_{1}-Z_{5}\right) v^{2}\right]^{2}+\left|Z_{6}\right|^{2} v^{4}}\right\}
\end{aligned}
$$

where $m_{h} \leq m_{H}$, and

$$
\begin{aligned}
s_{\beta-\alpha} c_{\beta-\alpha} & =\frac{-Z_{6} v^{2}}{m_{H}^{2}-m_{h}^{2}}, \\
c_{\beta-\alpha}^{2}-s_{\beta-\alpha}^{2} & =\frac{m_{A}^{2}-\left(Z_{1}-Z_{5}\right) v^{2}}{m_{H}^{2}-m_{h}^{2}} .
\end{aligned}
$$

We shall henceforth assume that $h \simeq \sqrt{2} \operatorname{Re} \mathcal{H}_{1}^{0}-v$ is SM-like and thus should be identified with the observed Higgs boson with $m_{h} \simeq 125 \mathrm{GeV}$. Under this assumption, it follows from Eq. (9.2) that $c_{\beta-\alpha} \rightarrow 0$ in the Higgs alignment limit. Indeed, one can use Eq. (9.4) to derive $[82,83]$

$$
c_{\beta-\alpha}=\frac{-Z_{6} v^{2}}{\sqrt{\left(m_{H}^{2}-m_{h}^{2}\right)\left(m_{H}^{2}-Z_{1} v^{2}\right)}}
$$

in a convention where $s_{\beta-\alpha} \geq 0$. Having identified $h$ as SM-like, it follows that $m_{H}^{2}>Z_{1} v^{2}$, which confirms that $c_{\beta-\alpha} \rightarrow 0$ in the Higgs alignment limit. ${ }^{24}$

One can now ask the following question-is there a symmetry that can be imposed on the 2HDM scalar potential such that the Higgs alignment limit is exact, corresponding to the condition that $Z_{6}=0$. In fact, it is straightforward to identify the complete list of all possible symmetries that enforce the $Z_{6}=0$ condition in the $2 \mathrm{HDM}$ by noting that the scalar potential minimum condition [cf. Eq. (5.20)] would then imply that $Y_{3}=0$. Thus, in light of Eq. (5.13), the Higgs alignment limit is exact if

$$
\begin{aligned}
Y_{3} & =\left[\frac{1}{2}\left(m_{22}^{2}-m_{11}^{2}\right) s_{2 \beta}-\operatorname{Re}\left(m_{12}^{2} e^{i \xi}\right) c_{2 \beta}-i \operatorname{Im}\left(m_{12}^{2} e^{i \xi}\right)\right] e^{-i \xi} \\
& =0
\end{aligned}
$$

A sufficient (but not necessary) condition for satisfying Eq. (9.6) can be obtained by setting $m_{12}^{2}=0$, in which case either $s_{2 \beta}=0$ or $m_{11}^{2}=m_{22}^{2}$.

If $s_{2 \beta}=0$, then the $\mathbb{Z}_{2}$ symmetry is unbroken by the vacuum. This corresponds to the IDM, where an unbroken $\mathbb{Z}_{2}$ symmetry is present in the Higgs basis, which implies that $Y_{3}=Z_{6}=Z_{7}=0$. In the IDM, the Higgs basis field $\mathcal{H}_{2}$ is odd under the $\mathbb{Z}_{2}$ symmetry, whereas $\mathcal{H}_{1}$ along with all other SM fields are $\mathbb{Z}_{2}$-even. If in addition, one imposes the condition $Z_{5}=0$, then the IDM scalar potential will exhibit an unbroken U(1) symmetry that is preserved by the vacuum (resulting in a mass-degenerate pair of inert neutral scalars, $m_{H}=m_{A}$ ). In both cases, one can identify $\varphi_{1}=\sqrt{2} \operatorname{Re} \mathcal{H}_{1}^{0}-v$ as the SM Higgs boson at tree level. Deviations of the properties of $\varphi_{1}$ from that of the SM Higgs boson can arise due to the other Higgs fields (beyond $\varphi_{1}$ ) contributing to radiative loop corrections to physical observables (e.g., the charged Higgs boson loop that contributes to $\varphi_{1} \rightarrow \gamma \gamma$ decay).

Note that if $m_{11}^{2}=m_{22}^{2}$ and $m_{12}^{2} \neq 0$ then one can achieve $Y_{3}=0$ by simultaneously imposing a $\Pi_{2}$ symmetry and a GCP1 symmetry in the $\Phi$-basis. Consulting Table III, these symmetries taken together yield the following constraints on the scalar potential:

$m_{11}^{2}=m_{22}^{2}, \quad m_{12}^{2}, \lambda_{5} \in \mathbb{R}, \quad \lambda_{1}=\lambda_{2}, \quad \lambda_{6}=\lambda_{7} \in \mathbb{R}$.

\footnotetext{
${ }^{24}$ If $H$ were SM-like (with $m_{H} \geq m_{h}$ ) then $m_{H}^{2} \rightarrow Z_{1} v^{2}$ in the Higgs alignment limit, in which case Eq. (9.5) would not be very useful. Indeed, in this case $s_{\beta-\alpha} \rightarrow 0$ in the Higgs alignment limit, and a more useful formula to replace Eq. (9.5) would be $s_{\beta-\alpha}=$ $-Z_{6} v^{2} / \sqrt{\left(m_{H}^{2}-m_{h}^{2}\right)\left(Z_{1} v^{2}-m_{h}^{2}\right)}$ in an alternative convention where $c_{\beta-\alpha} \geq 0$ [84]. However, in the conventions adopted in this paper $h$ is always SM-like in the Higgs alignment limit in light of Eq. (5.30), irrespective of the mass ordering of $h$ and $H$.
} 
Such a scalar potential does not lie within the ERPS4. One can now determine the scalar potential minimum conditions [see Eq. (E3) of Ref. [37] ], which yield

$$
c_{2 \beta}=0 \quad \text { and } \quad \sin \xi=0 .
$$

Hence, a scalar potential whose parameters satisfy Eqs. (9.7) and (9.8) yields $Y_{3}=0$ [cf. Eq. (9.6)], corresponding to an exact Higgs alignment [85]. Moreover, Eqs. (E1)-(E6) are satisfied after employing Eqs. (9.7) and (9.8), which implies that $Y_{3}=Z_{6}=Z_{7}=0$. That is, the scalar potential parameters of Eq. (9.7) corresponds to the IDM in a particular scalar field basis. Indeed, one can adopt a definition of the IDM scalar potential as corresponding to the existence of a scalar field basis in which the $\Pi_{2}$ and GCP1 symmetries are both manifestly preserved by the 2HDM scalar potential and vacuum.

If $s_{2 \beta} \neq 0$ then Eq. (9.6) is automatically satisfied if $m_{12}^{2}=0$ and $m_{11}^{2}=m_{22}^{2}$. Consulting the results of Table III, it follows that exact Higgs alignment is automatically implemented if the $2 \mathrm{HDM}$ scalar potential respects one of the following symmetries: $\mathbb{Z}_{2} \otimes \Pi_{2}, \mathrm{U}(1) \otimes \Pi_{2}, \mathrm{SO}(3)$, GCP2, or GCP3. Of course, given that GCP2 is equivalent to $\mathbb{Z}_{2} \otimes \Pi_{2}$ in a different scalar field basis and GCP3 is equivalent to $\mathrm{U}(1) \otimes \Pi_{2}$ in a different scalar field basis, it follows that there are three inequivalent symmetries of the 2HDM scalar potential beyond the IDM that yield exact Higgs alignment. What is common to these three inequivalent symmetries is that they all reside in the ERPS.

The conditions that $m_{11}^{2}=m_{22}^{2}$ and $m_{12}^{2}=0$ imply that the symmetries identified above are preserved by the scalar potential. In particular, in the ERPS (where $\lambda \equiv \lambda_{1}=\lambda_{2}$ and $\lambda_{7}=-\lambda_{6}$ ), Eq. (A10) of Ref. [37] yields

$$
\begin{aligned}
Z_{6}=e^{-i \xi} & \left\{-\frac{1}{2} s_{2 \beta} c_{2 \beta}\left(\lambda-\lambda_{345}\right)+\frac{1}{2} i s_{2 \beta} \operatorname{Im}\left(\lambda_{5} e^{2 i \xi}\right)\right. \\
& \left.+c_{4 \beta} \operatorname{Re}\left(\lambda_{6} e^{i \xi}\right)+i c_{2 \beta} \operatorname{Im}\left(\lambda_{6} e^{i \xi}\right)\right\},
\end{aligned}
$$

where $\lambda_{345} \equiv \lambda_{3}+\lambda_{4}+\operatorname{Re}\left(\lambda_{5} e^{2 i \xi}\right)$. We have already noted that the inert limit of the scalar potential in the ERPS regime, where exact Higgs alignment is achieved, corresponds to $Y_{3}=0$, which then implies that $Z_{6}=0$ via Eq. (5.20) and $Z_{7}=0$ due to the ERPS conditions. For example, in the case of a $\mathbb{Z}_{2} \otimes \Pi_{2}$-symmetric scalar potential, applying the conditions exhibited in Table III yields the expression for $Z_{6}$ given in Eq. (5.19). Because $m_{11}^{2}=m_{22}^{2}$ and $m_{12}^{2}=0$, it automatically follows that $Y_{3}=0$ which implies that $Z_{6}=0$. The vanishing of $Z_{6}$ [although not immediately evident from Eq. (9.9)] is a consequence of the scalar potential minimum conditions of the ERPS which yield

$$
\lambda(1-R) c_{2 \beta}=\lambda_{5} s_{2 \beta} \sin 2 \xi=0 \quad \text { or } \quad s_{2 \beta}=0,
$$

where $R \equiv\left(\lambda_{3}+\lambda_{4}+\lambda_{5}\right) / \lambda$. Since $R \neq 1$ and $\lambda_{5} \neq 0$ (otherwise, the symmetry group of the scalar potential is larger than $\mathbb{Z}_{2} \otimes \Pi_{2}$ ), it then follows that either $c_{2 \beta}=$ $\sin 2 \xi=0$ or $s_{2 \beta}=0$. Inserting these conditions in Eq. (9.9) along with the $\mathbb{Z}_{2} \otimes \Pi_{2}$ symmetry conditions, $\operatorname{Im} \lambda_{5}=\lambda_{6}=\lambda_{7}=0$, yields $Z_{6}=0$ as expected.

In the case of an unbroken $\mathrm{U}(1) \otimes \Pi_{2}$ symmetry, we simply add one additional condition, $\lambda_{5}=0$ to the scalar potential parameters (while maintaining $R \neq 1$ ). In this case, Eq. (9.10) implies that either $c_{2 \beta}=0$ or $s_{2 \beta}=0$ (with no restriction on $\xi$, which is an unphysical phase that can be rephased away), and again Eq. (9.9) yields $Z_{6}=0$.

It is instructive to consider the GCP3-symmetric scalar potential, which can be obtained from the $\mathbb{Z}_{2} \otimes \Pi_{2}$-symmetric scalar potential by adding one additional constraint, $R=1$ (while maintaining $\lambda_{5} \neq 0$ ). The case of an unbroken GCP3 symmetry is not physically distinct from the previous case since it is equivalent to a $\mathrm{U}(1) \otimes \Pi_{2}$ symmetry in a different scalar field basis. The minimum conditions of the GCP3-symmetric scalar potential (where we now employ primed parameters) yield

$\lambda_{5}^{\prime} c_{2 \beta^{\prime}} \sin ^{2} \xi^{\prime}=\lambda_{5}^{\prime} s_{2 \beta^{\prime}} \sin 2 \xi^{\prime}=0 \quad$ or $\quad s_{2 \beta^{\prime}}=0$.

These conditions guarantee that $Z_{6}=0$ [cf Eq. (7.13)], independently of the parameters of the GCP3-symmetric scalar potential. In particular, exact Higgs alignment is achieved for all values of $\beta^{\prime}$ in cases of an unbroken and some softly broken GCP3-symmetric scalar potentials, in contrast to the cases of $\mathbb{Z}_{2} \otimes \Pi_{2}$ and $\mathrm{U}(1) \otimes \Pi_{2}$ where exact Higgs alignment is satisfied only when $\beta=0, \frac{1}{4} \pi$ or $\frac{1}{2} \pi$. $^{25}$

In Refs. [39,40], Higgs alignment enforced by a symmetry is defined to be "natural" if $Z_{6}=0$ is achieved independently of the value of $\beta$. Based on the discussion above, this definition eliminates the $\mathbb{Z}_{2} \otimes \Pi_{2}$, GCP2 and $\mathrm{U}(1) \otimes \Pi_{2}$-symmetric scalar potentials from the list of potentials that exhibit a natural Higgs alignment. ${ }^{26}$

\footnotetext{
${ }^{25}$ The reader might wonder how it is possible that exact alignment can be achieved for all values of $\beta^{\prime}$ but only special values of $\beta$ in light of the fact that the $\mathrm{U}(1) \otimes \Pi_{2}$ and GCP3symmetric scalar potentials can be transformed into one another by an appropriate change of basis. The answer can be seen by examining Eq. (8.52). Employing Eq. (9.11) with $\lambda_{5}^{\prime} \neq 0$, it follows that either $\sin \xi^{\prime}=0$, in which case all values of $\beta^{\prime}$ are permitted, or $c_{2 \beta^{\prime}}=\cos \xi^{\prime}=0$. Using Eq. (8.52), it follows that the possible values of $\beta^{\prime}$ correspond to either $s_{2 \beta}=0$ or $c_{2 \beta}=0$.

${ }^{26}$ In Refs. [39,40], the maximal symmetry groups associated with the GCP3 and SO(3)-symmetric scalar potentials, which exhibit a natural Higgs alignment, are identified as $\mathbb{Z}_{2} \otimes \mathrm{O}(2) \otimes$ $\mathrm{O}(2)$ and $\mathrm{O}(3) \otimes \mathrm{O}(2)$, respectively. In addition, if the $\mathrm{U}(1)_{\mathrm{Y}}$ hypercharge gauge coupling $g^{\prime}=0$ in the gauge covariant kinetic terms of the scalar fields, then an $\mathrm{SO}(3)$-symmetric scalar potential with $\lambda_{4}=\lambda_{5}=0$ (cf. Table XII) yields an $\mathrm{SO}(5)$ symmetric scalar Lagrangian that also exhibits a natural Higgs alignment.
} 
In particular, the GCP3-symmetric scalar potential exhibits a natural alignment in the sense of Refs. [39,40], whereas the $\mathrm{U}(1) \otimes \Pi_{2}$-scalar potential does not. However, this distinction is problematical given that the GCP3-symmetric and $\mathrm{U}(1) \otimes \Pi_{2}$-symmetric scalar potentials are physically equivalent and can be transformed into each other by an appropriate change of scalar field basis (as shown explicitly in Sec. VIII). To avoid such an undesirable feature, a better definition of natural alignment in the spirit of Refs. $[39,40]$ would be to require that the conditions, $Y_{3}=Z_{6}=0$, should be independent of the scalar potential minimum conditions. Under this stricter definition, the GCP3symmetric scalar potential would not exhibit a natural Higgs alignment. In contrast, the $\mathrm{SO}(3)$-symmetric scalar potential with $m_{11}^{2}=m_{22}^{2}, m_{12}^{2}=0, \lambda_{1}=\lambda_{2}=\lambda_{3}+\lambda_{4}$ and $\lambda_{5}=\lambda_{6}=\lambda_{7}=0$ does satisfy $Y_{3}=Z_{6}=0$ independently of the scalar potential minimum conditions [cf. Eq. (9.9)] and thus would exhibit a natural Higgs alignment according to the stricter definition proposed above.

However, our preference is to employ the concept of naturalness as introduced by 't Hooft in Ref. [86], which implies that a small parameter of a theory should be considered natural if the symmetry of the Lagrangian is increased by setting the parameter to zero. In the present context, the small parameters are the potentially softbreaking parameters, $m_{11}^{2}-m_{22}^{2}$ and $m_{12}^{2}$, of the ERPS4 that could potentially generate departures from exact Higgs alignment. All symmetry groups of the ERPS-GCP2, $\mathbb{Z}_{2} \otimes \Pi_{2}, \mathrm{GCP} 3, \mathrm{U}(1) \otimes \Pi_{2}$ and $\mathrm{SO}(3)$-yield an exact Higgs alignment naturally in the sense of 't Hooft. Indeed, exact Higgs alignment realized in this way is stable under renormalization group running, which is further evidence that the symmetry based approach that we have adopted is correct. $^{27}$

The conditions that $m_{11}^{2}=m_{22}^{2}$ and $m_{12}^{2}=0$ are sufficient but not necessary for exact Higgs alignment. In particular, exact Higgs alignment arises in any inert limit of the 2HDM. Thus to obtain a complete classification of 2HDM scalar potentials that yield an exact Higgs alignment due to a symmetry, it suffices to enumerate the inert limits of the softly broken $\mathbb{Z}_{2} \otimes \Pi_{2}, \mathrm{U}(1) \otimes \Pi_{2}$ or GCP3, and $\mathrm{SO}(3)$-symmetric scalar potentials. ${ }^{28}$ These results can be found in Tables VII-X. We proceed to list all the relevant subcases below.

Given a softly broken $\mathbb{Z}_{2} \otimes \Pi_{2}$-symmetric scalar potential, exact Higgs alignment arises in two subcases, as shown in Sec. V: (i) $s_{2 \beta}=\sin 2 \xi=0, m_{11}^{2} \neq m_{22}^{2}$ and $m_{12}^{2}=0$,

\footnotetext{
${ }^{27}$ In general, renormalization group running does not preserve the scalar field basis. However, the group theoretic properties of the symmetries of the scalar potential, whose specific realization may change in different choices of the scalar field basis, do not depend on the basis choice.

${ }^{28}$ The possibility of natural Higgs alignment in the presence of soft symmetry-breaking squared mass terms has also been treated in Ref. [85].
}

which preserves a $\mathbb{Z}_{2}$ symmetry that is unbroken in the vacuum, and (ii) $c_{2 \beta}=\sin 2 \xi=0, \quad m_{11}^{2}=m_{22}^{2}$ and $\operatorname{Im}\left[m_{12}^{2}\right]^{2}=2 \operatorname{Re} m_{12}^{2} \operatorname{Im} m_{12}^{2}=0$, which preserves a $\Pi_{2}$ $\left[\Pi_{2}^{\prime}\right]$ symmetry if $\operatorname{Im} m_{12}^{2}=0\left[\operatorname{Re} m_{12}^{2}=0\right]$ that is unbroken in the vacuum. In the absence of soft breaking, the constraints on the scalar potential parameters due to $\Pi_{2}$ and $\Pi_{2}^{\prime}$ are identical. The $\mathbb{Z}_{2}, \Pi_{2}$ or $\Pi_{2}^{\prime}$ residual symmetries are responsible for maintaining the exact Higgs alignment.

Given a softly broken $\mathrm{U}(1) \otimes \Pi_{2}$-symmetric scalar potential, exact Higgs alignment arises in two subcases, as shown in Sec. VI: (i) $s_{2 \beta}=0, m_{11}^{2} \neq m_{12}^{2}$ and $m_{12}^{2}=0$, which preserves a $\mathrm{U}(1)$ symmetry that is unbroken in the vacuum, and (ii) $c_{2 \beta}=0, m_{11}^{2}=m_{22}^{2}$ and $m_{12}^{2} \neq 0$. In light of Eq. (6.4), one can rephase $\Phi_{2} \rightarrow e^{-i \xi} \Phi_{2}$ to achieve a real basis, in which case the scalar potential in subcase (ii) preserves a $\Pi_{2}$ symmetry that is unbroken in the vacuum. If one does not remove the (unphysical) parameter $\xi$, then Eq. (8.30) can be used to identify the unbroken vacuum symmetry as $\Pi_{2}^{(\xi)}$, which is $\Pi_{2}$ in the rephased scalar field basis. In the case of an unbroken $\mathrm{U}(1) \otimes \Pi_{2^{-}}$ symmetric scalar potential, the $\mathrm{U}(1) \otimes \Pi_{2}$ symmetry is spontaneously broken down to $\mathrm{U}(1)$ if $s_{2 \beta}=0$ or to $\Pi_{2}$ if $c_{2 \beta}=0$.

Although a softly broken GCP3-symmetric scalar potential is equivalent to a softly broken $\mathrm{U}(1) \otimes \Pi_{2}$-symmetric scalar potential in a different basis, it is instructive to enumerate the cases in which a softly broken GCP3symmetric scalar potential exhibits exact Higgs alignment. Using the results of Sec. VII, exact Higgs alignment arises in four subcases in terms of the primed GCP3 basis parameters: (i) $s_{2 \beta^{\prime}}=0, m_{11}^{\prime 2} \neq m_{22}^{\prime 2}$ and $m_{12}^{\prime 2}=0$, which preserves a $\mathbb{Z}_{2}$ symmetry that is unbroken in the vacuum; (ii) $c_{2 \beta^{\prime}}=\cos \xi^{\prime}=0, m_{11}^{\prime 2}=m_{22}^{\prime 2}$ and $\operatorname{Im} m_{12}^{\prime 2} \neq 0$, which preserves a $\mathrm{U}(1)^{\prime}$ symmetry that is unbroken in the vacuum; (iii) $c_{2 \beta^{\prime}}=\sin \xi^{\prime}=0, m_{11}^{\prime 2}=m_{22}^{\prime 2}$ and $\operatorname{Re} m_{12}^{\prime 2} \neq 0$, which preserves a $\Pi_{2}$ symmetry that is unbroken by the vacuum; and (iv) $s_{2 \beta^{\prime}} c_{2 \beta^{\prime}} \neq 0, \sin \xi^{\prime}=0, m_{11}^{\prime 2} \neq m_{22}^{\prime 2}$ and $\operatorname{Re} m_{12}^{\prime 2} \neq 0$, which preserves a $\bar{\Pi}_{2}^{(\alpha)}$ vacuum symmetry, where $\alpha=$ $\left(\frac{1}{2} \pi-2 \beta^{\prime}\right) \cos \xi^{\prime}= \pm\left(\frac{1}{2} \pi-2 \beta^{\prime}\right)$. This result is derived in Sec. VIII, where the $\bar{\Pi}_{2}^{(\alpha)}$ symmetry is introduced in Eq. (8.32) and the relation that yields $\alpha$ in terms of $\beta^{\prime}$ is obtained in Eq. (8.38). Finally, in the case of an unbroken GCP3-symmetric scalar potential, the GCP3 symmetry, which is equivalent to a $\mathrm{U}(1)^{\prime} \otimes \mathbb{Z}_{2}$ symmetry, is spontaneously broken down to $\mathrm{U}(1)^{\prime}\left[\mathbb{Z}_{2}\right]$ if $c_{2 \beta}=\cos \xi^{\prime}=0$ $\left[s_{2 \beta^{\prime}}=0\right]$, or to $\bar{\Pi}_{2}^{(\alpha)}$ with $\alpha= \pm\left(\frac{1}{2} \pi-2 \beta^{\prime}\right)$ if $s_{2 \beta^{\prime}} \neq 0$ and $\cos \xi^{\prime}= \pm 1$.

Finally, we examine the case of a softly broken $\mathrm{SO}(3)$ symmetric scalar potential. We noted above that in this case $Z_{6}=0$ independently of the scalar potential minimum conditions. This means that all softly broken $\mathrm{SO}(3)-$ symmetric scalar potentials exhibit exact Higgs alignment, 
since the scalar potential minimum conditions will guarantee that $Y_{3}=0$ even when $m_{11}^{2} \neq m_{22}^{2}$ and/or $m_{12}^{2} \neq 0$.

Below Eq. (6.25), we noted the presence of massdegenerate scalars, $H$ and $A$, which was attributed to a Peccei-Quinn $\mathrm{U}(1)$ symmetry in the Higgs basis, $\mathcal{H}_{1} \rightarrow \mathcal{H}_{1}, \mathcal{H}_{2} \rightarrow e^{2 i \theta} \mathcal{H}_{2}$ (for any value of $0 \leq \theta<\pi$ ), which is unbroken by the vacuum. ${ }^{29}$ It is instructive to ascertain the precise form of the $\mathrm{U}(1)$ symmetry in the $\Phi$-basis. To accomplish this, we employ Eq. (8.26), where the unitary matrix

$$
U=\left(\begin{array}{cc}
c_{\beta} & -e^{-i(\xi+\eta)} s_{\beta} \\
e^{i \xi} s_{\beta} & e^{-i \eta} c_{\beta}
\end{array}\right),
$$

transforms the Higgs basis into the $\Phi$-basis. The phase $e^{i \eta}$, which appears in Eq. (5.9) and reflects the freedom to rephase the Higgs basis field $\mathcal{H}_{2}$, cancels exactly when Eq. (8.26) is applied. Starting with $X=\left(\begin{array}{ll}1 & 0 \\ 0 & e^{2 i \theta}\end{array}\right)$, we make use of Eq. (8.26) with $\zeta=-\theta$ to obtain

$$
X^{\prime}=\left(\begin{array}{cc}
\cos \theta-i c_{2 \beta} \sin \theta & -i e^{-i \xi} s_{2 \beta} \sin \theta \\
-i e^{i \xi} s_{2 \beta} \sin \theta & \cos \theta+i c_{2 \beta} \sin \theta
\end{array}\right) .
$$

Thus, in the $\Phi$-basis characterized by $\tan \beta=\left|\left\langle\Phi_{2}^{0}\right\rangle /\left\langle\Phi_{1}^{0}\right\rangle\right|$ and $\xi=\arg \left[\left\langle\Phi_{1}^{0}\right\rangle *\left\langle\Phi_{2}^{0}\right\rangle\right]$, the Peccei-Quinn symmetry, which we designate by $\mathrm{U}(1)_{\mathrm{H}}$ (to remind the reader that it has been first applied in the Higgs basis), is given by

$$
\begin{aligned}
U(1)_{\mathrm{H}}: & \Phi_{1} \rightarrow\left(\cos \theta-i c_{2 \beta} \sin \theta\right) \Phi_{1}-i e^{-i \xi} s_{2 \beta} \sin \theta \Phi_{2} \\
\Phi_{2} & \rightarrow-i e^{i \xi} s_{2 \beta} \sin \theta \Phi_{1}+\left(\cos \theta+i c_{2 \beta} \sin \theta\right) \Phi_{2}
\end{aligned}
$$

Imposing the $\mathrm{U}(1)_{\mathrm{H}}$ symmetry on the parameters of a general $2 \mathrm{HDM}$ scalar potential in the $\Phi$-basis yields the following constraints:

$$
\begin{gathered}
\operatorname{Im}\left(m_{12}^{2} e^{i \xi}\right)=\lambda_{5}=\lambda_{6}=\lambda_{7}=0, \\
\lambda \equiv \lambda_{1}=\lambda_{2}=\lambda_{3}+\lambda_{4}, \\
m_{22}^{2}-m_{11}^{2}=2 \cot 2 \beta \operatorname{Re}\left(m_{12}^{2} e^{i \xi}\right) .
\end{gathered}
$$

These constraints correspond to a softly broken $\mathrm{SO}(3)$ symmetric scalar potential and scalar potential minimum conditions [cf. Eqs. (7.2)-(7.4) with $\lambda=\lambda_{3}+\lambda_{4}$ and $\left.\lambda_{5}=0\right]$. Moreover,

\footnotetext{
${ }^{29}$ The version of the Peccei-Quinn symmetry transformation that is used here corresponds to $\mathrm{U}(1)_{\mathrm{PQ}}$ given in Table I followed by a hypercharge $\mathrm{U}(1)_{\mathrm{Y}}$ transformation, which is also a symmetry of the vacuum in the Higgs basis.
}

$$
X^{\prime}\left(\begin{array}{c}
c_{\beta} \\
s_{\beta} e^{i \xi}
\end{array}\right)=e^{-i \theta}\left(\begin{array}{c}
c_{\beta} \\
s_{\beta} e^{i \xi}
\end{array}\right)
$$

which confirms that the vacuum is invariant under the $\mathrm{U}(1)_{\mathrm{H}}$ transformation [up to an overall hypercharge $\mathrm{U}(1)_{\mathrm{Y}}$ transformation that has no effect on the scalar potential parameters].

We conclude that for a generic softly broken $\mathrm{SO}(3)$ symmetric scalar potential, a $\mathrm{U}(1)_{\mathrm{H}}$ subgroup remains unbroken and is responsible for the mass degeneracy of $H$ and $A$ as well as the exact Higgs alignment. ${ }^{30}$ In the case of an unbroken $\mathrm{SO}(3)$-symmetric scalar potential, the $\mathrm{SO}(3)$ symmetry is spontaneously broken down to $\mathrm{U}(1)_{\mathrm{H}}$, in which case both $H$ and $A$ can be identified as massless Goldstone bosons (of opposite $C P$-quantum numbers).

This completes the classification of all unbroken or softly broken symmetries of the 2HDM scalar potential that yield an exact Higgs alignment. This classification is summarized in Table XI. Many aspects of this table can be easily understood by employing the results of Appendix E. Applying the ERPS4 conditions $\left(\lambda_{1}=\lambda_{2}\right.$ and $\lambda_{7}=-\lambda_{6}$ ) in Eqs. (E1)-(E6), the parameters of the scalar potential in the ERPS4 regime in the $\Phi$-basis satisfy

$$
\begin{gathered}
\operatorname{Im}\left(m_{12}^{2} e^{i \xi}\right)=0, \\
\left(m_{22}^{2}-m_{11}^{2}\right) s_{2 \beta}=2 \operatorname{Re}\left(m_{12}^{2} e^{i \xi}\right) c_{2 \beta}, \\
c_{4 \beta} \operatorname{Re}\left(\lambda_{6} e^{i \xi}\right)=\frac{1}{2} s_{2 \beta} c_{2 \beta}\left[\lambda-\lambda_{3}-\lambda_{4}-\operatorname{Re}\left(\lambda_{5} e^{2 i \xi}\right)\right], \\
c_{2 \beta} \operatorname{Im}\left(\lambda_{6} e^{i \xi}\right)=-\frac{1}{2} s_{2 \beta} \operatorname{Im}\left(\lambda_{5} e^{2 i \xi}\right) .
\end{gathered}
$$

Equations (9.19)-(9.22) provide the necessary and sufficient conditions for the inert limit of the scalar potential in the ERPS4 regime, thereby producing an exact Higgs alignment.

One can check that all the entries listed in Table XI [including the first two lines, which correspond respectively to the $\mathbb{Z}_{2}$-symmetric IDM and the U(1)-symmetric IDM, outside of the domain of the ERPS4] satisfy the four conditions specified in Eqs. (9.19)-(9.22). For example, starting from the softly broken or unbroken GCP2symmetric scalar potential transformed to the basis in which $\lambda_{5}$ is real and $\lambda_{6}=\lambda_{7}=0$, one easily obtains the following correlations of the parameters $\beta$ and $\xi$ for the symmetry cases listed in Table XI:

\footnotetext{
${ }^{30}$ In the case of $s_{2 \beta}=0$, the $\Phi$-basis coincides with the Higgs basis (up to a possible discrete $\Pi_{2}$ transformation), in which case $\mathrm{U}(1)_{\mathrm{H}}$ reduces to the standard $\mathrm{U}(1)_{\mathrm{PQ}}$ symmetry.
} 
TABLE XI. Classification of symmetries of the 2HDM scalar potential that yield exact Higgs alignment, where the tree-level properties of one of the neutral scalar mass eigenstates coincides with those of the SM Higgs boson. Note that $m_{11}^{2}=m_{22}^{2}$ and $\operatorname{Re}\left(m_{12}^{2} e^{i \xi}\right)=\operatorname{Im}\left(m_{12}^{2} e^{i \xi}\right)=0$ (and similarly for the primed parameters) unless otherwise indicated. The unprimed parameters correspond to the $\mathbb{Z}_{2} \otimes \Pi_{2}$ or $\mathrm{U}(1) \otimes \Pi_{2}$ basis, whereas the primed parameters correspond to the GCP3 basis. All such basis choices are consistent with the ERPS4 with $\lambda_{6}=\lambda_{7}=0$ and real $\lambda_{5}$; the corresponding parameter constraints for the softly broken GCP2symmetric scalar potential are given in Eqs. (9.19)-(9.22). In cases where the residual symmetry is given by $\bar{\Pi}_{2}^{(\alpha)}$, the value of $\alpha=\left(\frac{1}{2} \pi-2 \beta^{\prime}\right) \cos \xi^{\prime}$, where $\cos \xi^{\prime}= \pm 1$. Although separate listings are provided for scalar potentials that exhibit the $\mathrm{U}(1) \otimes \Pi_{2}$ or GCP3 symmetry (either of which may be softly broken), they represent the same scalar potential expressed in two different choices of the scalar field basis.

\begin{tabular}{|c|c|c|c|c|}
\hline \multirow[b]{2}{*}{ Symmetry } & \multirow[b]{2}{*}{ Soft breaking } & \multirow[b]{2}{*}{ Parameter constraints } & \multicolumn{2}{|c|}{ Residual unbroken symmetry of } \\
\hline & & & Scalar potential & Vacuum \\
\hline $\mathbb{Z}_{2}$ & None & $s_{2 \beta}=0$ & $\mathbb{Z}_{2}$ & $\mathbb{Z}_{2}$ \\
\hline $\mathrm{U}(1)$ & None & $s_{2 \beta}=0$ & $\mathrm{U}(1)$ & $\mathrm{U}(1)$ \\
\hline $\mathbb{Z}_{2} \otimes \Pi_{2}$ & $m_{11}^{2} \neq m_{22}^{2}$ & $s_{2 \beta}=0$ & $\mathbb{Z}_{2}$ & $\mathbb{Z}_{2}$ \\
\hline $\mathbb{Z}_{2} \otimes \Pi_{2}$ & $\operatorname{Re} m_{12}^{2} \neq 0$ & $c_{2 \beta}=\sin \xi=0$ & $\Pi_{2}$ & $\Pi_{2}$ \\
\hline $\mathbb{Z}_{2} \otimes \Pi_{2}$ & $\operatorname{Im} m_{12}^{2} \neq 0$ & $c_{2 \beta}=\cos \xi=0$ & $\Pi_{2}^{\prime}$ & $\Pi_{2}^{\prime}$ \\
\hline $\mathbb{Z}_{2} \otimes \Pi_{2}$ & None & $s_{2 \beta}=0$ & $\mathbb{Z}_{2} \otimes \Pi_{2}$ & $\mathbb{Z}_{2}$ \\
\hline $\mathbb{Z}_{2} \otimes \Pi_{2}$ & None & $c_{2 \beta}=\sin 2 \xi=0$ & $\mathbb{Z}_{2} \otimes \Pi_{2}$ & $\Pi_{2}$ \\
\hline $\mathrm{U}(1) \otimes \Pi_{2}$ & $m_{11}^{2} \neq m_{22}^{2}$ & $s_{2 \beta}=0$ & $\mathrm{U}(1)$ & $\mathrm{U}(1)$ \\
\hline $\mathrm{U}(1) \otimes \Pi_{2}$ & $\operatorname{Re}\left(m_{12}^{2} e^{i \xi}\right) \neq 0$ & $c_{2 \beta}=0$ & $\Pi_{2}^{(\xi)}$ & $\Pi_{2}^{(\xi)}$ \\
\hline $\mathrm{U}(1) \otimes \Pi_{2}$ & None & $s_{2 \beta}=0$ & $\mathrm{U}(1) \otimes \Pi_{2}$ & $\mathrm{U}(1)$ \\
\hline $\mathrm{U}(1) \otimes \Pi_{2}$ & None & $c_{2 \beta}=0$ & $\mathrm{U}(1) \otimes \Pi_{2}$ & $\Pi_{2}$ \\
\hline GCP3 & $m_{11}^{\prime 2} \neq m_{22}^{\prime 2}, \operatorname{Re} m_{12}^{\prime 2} \neq 0$ & $s_{2 \beta^{\prime}} c_{2 \beta^{\prime}} \neq 0, \sin \xi^{\prime}=0$ & $\bar{\Pi}_{2}^{(\alpha)}$ & $\bar{\Pi}_{2}^{(\alpha)}$ \\
\hline GCP3 & $m_{11}^{\prime 2} \neq m_{22}^{\prime 2}$ & $s_{2 \beta^{\prime}}=0$ & $\mathbb{Z}_{2}$ & $\mathbb{Z}_{2}$ \\
\hline GCP3 & $\operatorname{Re} m_{12}^{\prime 2} \neq 0$ & $c_{2 \beta^{\prime}}=0, \sin \xi^{\prime}=0$ & $\Pi_{2}$ & $\Pi_{2}$ \\
\hline GCP3 & $\operatorname{Im} m_{12}^{\prime 2} \neq 0$ & $c_{2 \beta^{\prime}}=0, \cos \xi^{\prime}=0$ & $\mathrm{U}(1)^{\prime}$ & $\mathrm{U}(1)^{\prime}$ \\
\hline GCP3 & None & $s_{2 \beta^{\prime}}=0$ & $\mathrm{U}(1)^{\prime} \otimes \mathbb{Z}_{2}$ & $\mathbb{Z}_{2}$ \\
\hline GCP3 & None & $s_{2 \beta^{\prime}} \neq 0, \sin \xi^{\prime}=0$ & $\mathrm{U}(1)^{\prime} \otimes \mathbb{Z}_{2}$ & $\bar{\Pi}_{2}^{(\alpha)}$ \\
\hline GCP3 & None & $c_{2 \beta^{\prime}}=0, \cos \xi^{\prime}=0$ & $\mathrm{U}(1)^{\prime} \otimes \mathbb{Z}_{2}$ & $\mathrm{U}(1)^{\prime}$ \\
\hline $\mathrm{SO}(3)$ & $m_{11}^{\prime 2} \neq m_{22}^{\prime 2}, \operatorname{Re}\left(m_{12}^{\prime 2} e^{i \xi^{\prime}}\right) \neq 0$ & $s_{2 \beta^{\prime}} c_{2 \beta^{\prime}} \neq 0$ & $\mathrm{U}(1)_{\mathrm{H}}$ & $\mathrm{U}(1)_{\mathrm{H}}$ \\
\hline $\mathrm{SO}(3)$ & $\operatorname{Re}\left(m_{12}^{\prime 2} e^{i \xi^{\prime}}\right) \neq 0$ & $c_{2 \beta^{\prime}}=0$ & $\mathrm{U}(1)_{\mathrm{H}}$ & $\mathrm{U}(1)_{\mathrm{H}}$ \\
\hline $\mathrm{SO}(3)$ & $m_{11}^{\prime 2} \neq m_{22}^{\prime 2}$ & $s_{2 \beta^{\prime}}=0$ & $\mathrm{U}(1)$ & $\mathrm{U}(1)$ \\
\hline $\mathrm{SO}(3)$ & None & None & $\mathrm{SO}(3)$ & $\mathrm{U}(1)_{\mathrm{H}}$ \\
\hline
\end{tabular}

$$
\begin{gathered}
\mathbb{Z}_{2} \otimes \Pi_{2}: s_{2 \beta} s_{2 \xi}=0 \quad \text { or } \quad s_{2 \beta} c_{2 \beta}=0, \\
\mathrm{U}(1) \otimes \Pi_{2}: s_{2 \beta} c_{2 \beta}=0, \\
\mathrm{GCP} 3: s_{2 \beta^{\prime}} s_{2 \xi^{\prime}}=0,
\end{gathered}
$$$$
\mathrm{SO}(3) \text { : no constraints. }
$$

One can then employ Eqs. (9.19) and (9.20) to determine the allowed soft breaking due to $m_{11}^{2} \neq m_{22}^{2}$ and/or $m_{12}^{2} \neq 0$ that is consistent with exact Higgs alignment.

\section{IMPLICATIONS OF CUSTODIAL SYMMETRY}

One of the possible symmetries of the scalar potential that does not appear in Table III is custodial symmetry.
This symmetry is necessarily violated by the hypercharge $\mathrm{U}(1)_{\mathrm{Y}}$ gauge interactions. ${ }^{31}$ Nevertheless, in the limit of $g^{\prime} \rightarrow 0$ this is a potential symmetry of the bosonic sector of the 2HDM. Given that custodial symmetry is an approximate symmetry of nature in light of the small deviation of the electroweak $\rho$-parameter from its custodial symmetric value of $\rho=1$, it is of interest to consider the possibility that the 2HDM scalar potential respects the custodial symmetry. In more detail, if the $2 \mathrm{HDM}$ scalar potential is symmetric under $\mathrm{SU}(2)_{L} \otimes \mathrm{SU}(2)_{R}$ transformations where $\mathrm{SU}(2)_{L}$ is identified with the $\mathrm{SU}(2)$ part of the electroweak gauge group and $\mathrm{SU}(2)_{R}$ is a global symmetry group, then after the symmetry breaking of $\mathrm{SU}(2)_{L}$ the residual custodial symmetry can be identified with an unbroken diagonal $\mathrm{SU}(2)_{L+R}$ global symmetry.

\footnotetext{
${ }^{31}$ That is, the custodial symmetry is violated by the gauge covariant kinetic term of the scalar fields that is proportional to $g^{\prime}$.
} 
Details of the $\mathrm{SU}(2)_{L} \otimes \mathrm{SU}(2)_{R}$ transformation laws are given in Refs. [50,52-54]. As shown in Ref. [52], a $\Phi$-basis exists for any $2 \mathrm{HDM}$ custodial symmetric scalar potential such that ${ }^{32}$

$$
\lambda_{4}=\operatorname{Re} \lambda_{5} \quad \text { and } \quad m_{12}^{2}, \lambda_{5}, \lambda_{6}, \quad \lambda_{7} \in \mathbb{R} .
$$

Hence, all 2HDM custodial symmetric scalar potentials are explicitly $C P$ conserving. In the case of an unbroken $\mathbb{Z}_{2}$-symmetric scalar potential where $m_{12}^{2}=\lambda_{6}=\lambda_{7}=0$, one is always free to rephase $\Phi_{2} \rightarrow i \Phi_{2},{ }^{33}$ in which case the custodial symmetry condition of Eq. (10.1) specializes to

$\lambda_{4}= \pm \operatorname{Re} \lambda_{5} \quad$ and $\quad m_{12}^{2}=\operatorname{Im} \lambda_{5}=\lambda_{6}=\lambda_{7}=0$.

Additional information is provided by minimizing the scalar potential and determining the Higgs basis. Then, as shown in Ref. [53], the scalar potential respects the custodial symmetry if the Higgs basis parameters satisfy

$$
\begin{aligned}
Z_{4}=Z_{5} e^{-2 i \eta} & \in \mathbb{R}, \quad Y_{3} e^{-i \eta}=-\frac{1}{2} Z_{6} e^{-i \eta} v^{2} \in \mathbb{R}, \\
Z_{7} e^{-i \eta} & \in \mathbb{R},
\end{aligned}
$$

where the phase $\eta$ represents the freedom to rephase the Higgs basis field $\mathcal{H}_{2}$. It follows that one can choose $\eta$ such that the parameters of the scalar potential in the Higgs basis are all real, which implies that GCP1 is a symmetry of the scalar potential and vacuum. In particular, in a real Higgs basis, Eq. (10.3) yields two possible solutions,

$$
\begin{gathered}
Z_{4}=Z_{5}, \\
\quad \text { or } \\
Z_{4}= \pm Z_{5}, \quad \text { and } Y_{3}=Z_{6}=Z_{7}=0 .
\end{gathered}
$$

Equation (10.4) is a consequence of choosing $\eta=0$ $(\bmod \pi)$. In the case of $Y_{3}=Z_{6}=Z_{7}=0$, the condition $Z_{4}=-Z_{5}$ is now possible by choosing $\eta=\frac{1}{2} \pi(\bmod \pi)$, as indicated in Eq. (10.5). Note that if the Yukawa interactions are neglected then the sign of $Z_{5}$ in a real Higgs basis is not physical since one can always redefine $\mathcal{H}_{2} \rightarrow i \mathcal{H}_{2}$ while maintaining the reality of the Higgs basis. Thus, Eq. (10.5) can be understood to mean that $Z_{4}= \pm\left|Z_{5}\right|$ in a real Higgs

\footnotetext{
${ }^{32}$ If $\lambda_{4}=\lambda_{5}$ in the $\Phi$-basis where $\lambda_{5} \in \mathbb{R}$, then $\lambda_{4}=\lambda_{5}$ in any real basis, $\Phi^{\prime}=U \Phi$, such that $U$ is a real orthogonal matrix. However, if $m_{12}^{2}=\lambda_{6}=\lambda_{7}=0$, then one can perform a basis transformation where $U$ is unitary but not real orthogonal that still preserves the reality of the basis. For such basis transformations the relation $\lambda_{4}=\lambda_{5}$ is no longer preserved. Equation (10.2) provides a trivial example of this. A more interesting example is provided by the basis transformation that converts Eq. (10.10) into Eq. (10.11). Of course, if $U$ is not real orthogonal, then the basis transformation will not preserve the reality of the vevs.

${ }^{33}$ Although this rephasing maintains the reality of the scalar potential parameters, it introduces a relative phase, $\xi=\frac{1}{2} \pi$, in the vevs.
}

basis, which corresponds to two physically inequivalent conditions.

Moreover, employing the results of Eq. (10.3) in Eqs. (5.21)-(5.23), it follows that if $Z_{6} \neq 0$ then we can identify the squared mass of the $C P$-odd mass eigenstate as corresponding to the 33 element of the squared-mass matrix $\mathcal{M}^{2}$ given in Eq. (5.22), namely $m_{A}^{2}=m_{H^{ \pm}}^{2}$. If $Z_{6}=0$, then $\mathcal{M}^{2}$ is diagonal; nevertheless, one can determine the $C P$ properties of the neutral Higgs mass eigenstates via the three-scalar and four-scalar interaction terms assuming that $Z_{7} \neq 0$ [69]. One can again confirm that the $C P$-odd mass eigenstate corresponds to the 33 element of $\mathcal{M}^{2}$, in which case we also conclude that $m_{A}^{2}=m_{H^{ \pm}}^{2}$.

Finally, in the case of a custodially symmetric scalar potential with $Y_{3}=Z_{6}=Z_{7}=0$, an exact Higgs alignment is realized and we can identify $m_{h}^{2}=Z_{1} v^{2}$ following the convention of Eq. (5.30), and $m_{H, A}^{2}=$ $m_{H^{ \pm}}^{2}+\frac{1}{2}\left(Z_{4} \pm Z_{5}\right) v^{2}$. Although the $C P$-quantum numbers of $H$ and $A$ are of opposite sign, there are no bosonic interactions that can uniquely identify which of the two states $H$ and $A$ is $C P$ even and which is $C P$ odd, as previously noted in Sec. V. Ultimately, the $C P$-quantum numbers of $H$ and $A$ may be fixed by the Higgs-fermion Yukawa couplings (if these interactions are $C P$ conserving), except in special cases where the ambiguity persists [cf. Eq. (5.31)]. Assuming that the $C P$-quantum numbers of $H$ and $A$ are unambiguously determined by the Yukawa couplings, then the sign of $Z_{5}$ is promoted to a physical parameter in the case of $Y_{3}=Z_{6}=Z_{7}=0$. It then follows that [53]

$$
m_{H^{ \pm}}^{2}= \begin{cases}m_{A}^{2} & \text { if } Z_{4}=Z_{5} \text { and } Z_{6}=Z_{7}=0, \\ m_{H}^{2} & \text { if } Z_{4}=-Z_{5} \text { and } Z_{6}=Z_{7}=0,\end{cases}
$$

in a real Higgs basis. In particular,

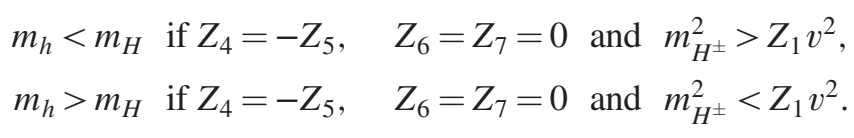

Indeed, the transformation $\mathcal{H}_{2} \rightarrow i \mathcal{H}_{2}$ changes the sign of $Z_{5}$ while also changing the scalar Yukawa coupling into a pseudoscalar Yukawa coupling and vice versa.

In this section, we propose to classify all $2 \mathrm{HDM}$ custodial-symmetric scalar potentials that exhibit exact Higgs alignment due to an unbroken or softly broken symmetry. All such scalar potentials will satisfy the Higgs basis conditions given in Eq. (10.5). If the parameters of the corresponding Higgs potential lie in the ERPS4 regime, then this classification amounts to supplementing the results of Table XI with the conditions of custodial symmetry.

As a first step, we review the classification of custodial symmetric 2HDM scalar potentials first obtained in Refs. [59,87] (and recently reproduced in Ref. [88]). If 
the scalar potential respects a custodial symmetry, then the Higgs Lagrangian can exhibit seven additional global symmetries in the limit of $g^{\prime}=0$ beyond the symmetries listed in Tables I and II. Three of the seven symmetries correspond to GCP1, the Peccei-Quinn U(1) and $\Pi_{2}$, which when combined with the custodial symmetry yield maximal symmetry groups of $\mathrm{SO}(3), \mathrm{SO}(4)$ and $\mathbb{Z}_{2} \otimes \mathrm{O}(3)$, respectively [87]. The case of GCP1 corresponds to the minimal implementation of custodial symmetry in the most general 2HDM scalar potential. Indeed, the custodialsymmetric scalar potential of the $2 \mathrm{HDM}$ must be $C P$ conserving as noted below Eq. (10.2). A custodial symmetric, $\Pi_{2}$-symmetric scalar potential is equivalent to a custodial symmetric, $\mathbb{Z}_{2}$ symmetric scalar potential in another scalar field basis. To validate this remark, we first combine Eq. (10.1) with the constraints of the $\Pi_{2}$ symmetry shown in Table III to obtain

$$
\begin{array}{rlr}
m_{11}^{2} & =m_{22}^{2}, \quad \lambda_{1}=\lambda_{2}, \quad \lambda_{4}=\operatorname{Re} \lambda_{5}, \quad \lambda_{6}=\lambda_{7}, \\
\operatorname{Im} m_{12}^{2} & =\operatorname{Im} \lambda_{5}=\operatorname{Im} \lambda_{6}=\operatorname{Im} \lambda_{7}=0,
\end{array}
$$

in the $\Phi$-basis. We now transform to a new basis by defining $\Phi_{1}^{\prime}=\left(\Phi_{1}+\Phi_{2}\right) / \sqrt{2}$ and $\Phi_{2}^{\prime}=\left(\Phi_{2}-\Phi_{1}\right) / \sqrt{2}$. In this new basis, the corresponding scalar potential parameters are

$$
\begin{aligned}
m_{11}^{\prime 2} & =m_{11}^{2}-\operatorname{Re} m_{12}^{2}, \quad m_{22}^{\prime 2}=m_{11}^{2}+\operatorname{Re} m_{12}^{2}, \quad m_{12}^{\prime 2}=0, \\
\lambda_{1}^{\prime} & =\frac{1}{2}\left(\lambda_{1}+\lambda_{3}+2 \lambda_{4}+4 \lambda_{6}\right), \\
\lambda_{2}^{\prime} & =\frac{1}{2}\left(\lambda_{1}+\lambda_{3}+2 \lambda_{4}-4 \lambda_{6}\right), \\
\lambda_{3}^{\prime} & =\frac{1}{2}\left(\lambda_{1}+\lambda_{3}-2 \lambda_{4}\right), \quad \lambda_{4}^{\prime}=\operatorname{Re} \lambda_{5}^{\prime}=\frac{1}{2}\left(\lambda_{1}-\lambda_{3}\right), \\
\operatorname{Im} \lambda_{5}^{\prime} & =\lambda_{6}^{\prime}=\lambda_{7}^{\prime}=0,
\end{aligned}
$$

which combines the constraints of Eq. (10.1) with the constraints of the $\mathbb{Z}_{2}$ symmetry.

The remaining four symmetry cases of Ref. [87] correspond to $\mathbb{Z}_{2} \otimes \Pi_{2}, \mathrm{U}(1) \otimes \Pi_{2}, \mathrm{GCP} 3$ and $\mathrm{SO}(3)$, which when combined with custodial symmetry [cf. Eq. (10.2)] yields a maximal symmetry group of $\mathbb{Z}_{2} \otimes \mathbb{Z}_{2} \otimes \mathrm{SO}(3)$, $\mathrm{O}(2) \otimes \mathrm{O}(3), \mathbb{Z}_{2} \otimes \mathrm{O}(4)$ and $\mathrm{SO}(5)$, respectively. ${ }^{34}$ In light of Eq. (9.6), each of these symmetry cases corresponds to the inert limit in the ERPS, and thus satisfy Eq. (10.5) in a real Higgs basis. The case of $\mathrm{O}(2) \otimes \mathrm{O}(3)$

\footnotetext{
${ }^{34}$ Since a GCP3-symmetric scalar potential is a $U(1) \otimes \Pi_{2}$ symmetric scalar potential in a different scalar field basis, one cannot unambiguously associate $\mathrm{O}(2) \otimes \mathrm{O}(3)$ and $\mathbb{Z}_{2} \otimes \mathrm{O}(4)$ with either ERPS symmetry. A physical criterion for distinguishing these two maximal symmetry groups is provided by the two Higgs basis conditions specified in Eq. (10.19) and exhibited in Table XII and in the discussion that follows.
}

requires some clarification. In Table 1 of Ref. [87], the constraints on the scalar potential parameters corresponding to the $\mathrm{O}(2) \otimes \mathrm{O}(3)$ symmetry are

$$
\begin{aligned}
m_{11}^{2} & =m_{22}^{2}, \quad m_{12}^{2}=0, \quad \lambda_{1}=\lambda_{2}=\lambda_{3}, \\
\lambda_{5} & =\lambda_{6}=\lambda_{7}=0 .
\end{aligned}
$$

This is a $\mathrm{U}(1) \otimes \Pi_{2}$-symmetric scalar potential, but it does not satisfy Eq. (10.1). However, if we transform to the GCP3 basis then Eqs. (8.2)-(8.9) yield

$$
\begin{aligned}
m_{11}^{\prime 2} & =m_{22}^{\prime 2}, \quad m_{12}^{\prime 2}=0, \quad \lambda_{1}^{\prime}=\lambda_{2}^{\prime}=\lambda_{3}^{\prime}+\lambda_{4}^{\prime}+\operatorname{Re} \lambda_{5}^{\prime}, \\
\lambda_{4}^{\prime} & =\operatorname{Re} \lambda_{5}^{\prime}, \quad \operatorname{Im} \lambda_{5}^{\prime}=\lambda_{6}^{\prime}=\lambda_{7}^{\prime}=0, \quad(10.11)
\end{aligned}
$$

which corresponds to custodial-symmetric, GCP3-symmetric scalar potential.

We are now ready to present the classification of custodial-symmetric 2HDM scalar potentials that satisfy exact Higgs alignment due to an unbroken or softly broken symmetry. Exact Higgs alignment requires $Y_{3}=Z_{6}=0$, and then to achieve Higgs alignment via a symmetry also requires $Z_{7}=0$. Two immediate examples are the IDM with either an unbroken $\mathbb{Z}_{2}$ or U(1) symmetry in the Higgs basis. Supplementing these two examples with the condition that $Z_{4}= \pm Z_{5}$ yields a custodial symmetric scalar potential with exact Higgs alignment.

In light of the classification of custodial symmetric scalar potentials discussed above, we now consider cases in which additional unbroken or softly broken symmetries are present. It is clear that at minimum, a softly broken $\mathbb{Z}_{2}$ symmetry that is manifestly realized in the $\Phi$-basis must be present. Consequently, let us consider a softly broken $\mathbb{Z}_{2}$-symmetric scalar potential in the $\Phi$-basis that is distinct from the Higgs basis which satisfies $Y_{3}=Z_{6}=Z_{7}=0$. Since $\lambda_{6}=\lambda_{7}=0$ holds in a $\Phi$-basis such that $s_{2 \beta} \neq 0$, the ERPS4 conditions must be satisfied as we now demonstrate.

First, we shall employ Eqs. (A.26)-(A.28) of Ref. [37] with $s_{2 \beta} \neq 0, \lambda_{6}=\lambda_{7}=0$ and $Z_{6}=Z_{7}=0$ to obtain

$$
\begin{aligned}
& s_{2 \beta}\left[Z_{1} c_{\beta}^{2}-Z_{2} s_{\beta}^{2}-Z_{34} c_{2 \beta}-\operatorname{Re}\left(Z_{5} e^{2 i \xi}\right) c_{2 \beta}-i \operatorname{Im}\left(Z_{5} e^{2 i \xi}\right)\right] \\
& \quad=0, \\
& s_{2 \beta}\left[Z_{1} s_{\beta}^{2}-Z_{2} c_{\beta}^{2}+Z_{34} c_{2 \beta}+\operatorname{Re}\left(Z_{5} e^{2 i \xi}\right) c_{2 \beta}+i \operatorname{Im}\left(Z_{5} e^{2 i \xi}\right)\right] \\
& \quad=0,
\end{aligned}
$$

where $Z_{34} \equiv Z_{3}+Z_{4}$. Adding and subtracting these two equations yields

$$
\begin{aligned}
& s_{2 \beta}\left(Z_{1}-Z_{2}\right)=0, \\
& s_{2 \beta}\left\{c_{2 \beta}\left[Z_{1}+Z_{2}-2 Z_{34}-2 \operatorname{Re}\left(Z_{5} e^{2 i \xi}\right)\right]-2 i \operatorname{Im}\left(Z_{5} e^{2 i \xi}\right)\right\} \\
& =0 .
\end{aligned}
$$


Moreover, we can use Eqs. (A20) of Ref. [37] along with Eq. (5.20) to obtain

$$
m_{12}^{2} e^{i \xi}=\frac{1}{2}\left(Y_{2}-Y_{1}\right) s_{2 \beta} .
$$

It follows that

$$
\operatorname{Im}\left(m_{12}^{2} e^{i \xi}\right)=0
$$

Imposing the scalar potential minimum condition given by Eq. (2.10), it follows that either $\sin 2 \xi=0$ or $\lambda_{5}=0$. If $\lambda_{5}=0$ then the only remaining complex parameter of the scalar potential, $m_{12}^{2}$, can be arbitrarily rephased. Thus without loss of generality, we may assume that $\sin 2 \xi=0$ holds in all cases, or equivalently $e^{2 i \xi}= \pm 1$. Moreover, having assumed that $s_{2 \beta} \neq 0$, we see that Eqs. (10.14) and (10.15) yields $Z_{1}=Z_{2}$ and $\operatorname{Im} Z_{5}=0$. That is, the Higgs basis parameters satisfy the ERPS4 conditions. ${ }^{35}$

In the case of $s_{2 \beta} c_{2 \beta} \neq 0$ and $e^{2 i \xi}= \pm 1$ (and $Z_{6}=Z_{7}=0$ ), Eqs. (10.14) and (10.15) yield

$$
Z_{1}=Z_{2}=Z_{3}+Z_{4} \pm Z_{5} \quad \text { and } \quad \operatorname{Im} Z_{5}=0 .
$$

Hence, the quartic terms of the scalar potential satisfy the GCP3 or GCP3' symmetry conditions in the Higgs basis. If we now also impose the custodial symmetry condition, $Z_{4}= \pm Z_{5}$, where the sign choice is uncorrelated with the \pm sign appearing in Eq. (10.18), then it follows that two relations are possible that are physically distinguishable,

$$
Z_{1}=Z_{2}=Z_{3}+2 Z_{4} \quad \text { or } \quad Z_{1}=Z_{2}=Z_{3} .
$$

Moreover, we can use Eqs. (A21)-(A28) of Ref. [37] along with Eq. (10.18) to obtain the scalar potential parameters in the $\Phi$-basis,

$$
\lambda_{i}=Z_{i} \quad \text { for } i=1,2, \ldots, 7 \text {. }
$$

When $Y_{3}=Z_{6}=Z_{7}=0$, it is always possible to rephase the Higgs basis field, $\mathcal{H}_{2} \rightarrow i \mathcal{H}_{2}$, such that $Z_{1}=Z_{2}=Z_{3}+Z_{4}+Z_{5}$. Then, in a GCP3 basis (where parameters in the $\Phi$-basis are indicated with prime superscripts), Eqs. (10.19) and (10.20) respectively yield

$$
\begin{aligned}
\lambda_{1}^{\prime}=\lambda_{2}^{\prime}=\lambda_{3}^{\prime}+\lambda_{4}^{\prime}+\lambda_{5}^{\prime}, & \text { where } \lambda_{4}^{\prime}=\lambda_{5}^{\prime}, \\
& \text { or } \\
\lambda_{1}^{\prime}=\lambda_{2}^{\prime}=\lambda_{3}^{\prime}, & \text { where } \lambda_{4}^{\prime}=-\lambda_{5}^{\prime} .
\end{aligned}
$$

\footnotetext{
${ }^{35}$ As expected, if $s_{2 \beta}=0$ then Eqs. (10.14) and (10.15) are automatically satisfied, in which case no enhanced symmetry beyond $\mathbb{Z}_{2}$ is present for generic parameters of the scalar potential.
}

In both cases, the GCP3 conditions are manifestly realized by the quartic terms of the scalar potential in the $\Phi$-basis. In light of Eq. (10.11), in the case of unbroken GCP3 and custodial symmetry, Eq. (10.21) yields a maximal symmetry group of $\mathrm{O}(2) \otimes \mathrm{O}(3)$ in the classification of Ref. [87]. To determine the maximal symmetry group of the scalar potential whose parameters satisfy Eq. (10.22), we transform to the $\mathrm{U}(1) \otimes \Pi_{2}$ basis. Then Eqs. (8.42)-(8.46) yield $\lambda_{1}=\lambda_{2} \neq \lambda_{3}$ and $\lambda_{4}=\lambda_{5}=\lambda_{6}=$ $\lambda_{7}=0$, corresponding to a maximal symmetry group of $\mathbb{Z}_{2} \otimes \mathrm{O}(4)$ in the classification of Ref. [87]. Note that the custodial symmetry is preserved in the presence of soft breaking of the GCP3 symmetry by allowing for $m_{12}^{\prime 2} \neq 0$ subject to the condition, $m_{22}^{\prime 2}-m_{11}^{\prime 2}=2 m_{12}^{\prime 2} c_{2 \beta^{\prime}} / s_{2 \beta^{\prime}}$ [cf. Eqs. (7.2) and (7.3)].

If $Z_{5}=0$, then Eq. (10.20) together with the custodial symmetry condition $Z_{4}= \pm Z_{5}$ imply that $\lambda_{4}^{\prime}=\lambda_{5}^{\prime}=0$. In light of Eqs. (10.21) and (10.22), the $\mathrm{SO}(3)$ conditions are manifestly realized by the quartic terms of the scalar potential. Indeed, in this case the quartic terms are given by $\mathcal{V} \ni \frac{1}{2} \lambda\left(\Phi_{1}^{\dagger} \Phi_{1}+\Phi_{2}^{\dagger} \Phi_{2}\right)^{2}$, which corresponds to the maximally symmetric $\mathrm{SO}(5)$ limit of the 2HDM (after including the gauge covariant kinetic terms of the scalar fields with $g^{\prime}=0$ ) analyzed in Ref. [39]. Soft breaking of the $\mathrm{SO}(3)$ symmetry due to $m_{12}^{\prime 2} \neq 0$ is again allowed subject to the condition, $m_{22}^{\prime 2}-m_{11}^{\prime 2}=2 m_{12}^{\prime 2} c_{2 \beta^{\prime}} / s_{2 \beta^{\prime}}$.

In the case of $c_{2 \beta}=\sin 2 \xi=0$, Eqs. (10.14) and (10.15) yield $Z_{1}=Z_{2}$ and $\operatorname{Im} Z_{5}=0$. Hence, the quartic terms of the scalar potential in the real Higgs basis satisfy the $\mathbb{Z}_{2} \otimes \Pi_{2}$ symmetry conditions. Using Eqs. (A21)-(A25) of Ref. [37], we obtain

$$
\begin{aligned}
& \lambda_{1}=\lambda_{2}=Z_{1}-\frac{1}{2}\left(Z_{1}-Z_{345}\right), \\
& \lambda_{i}=Z_{i}+\frac{1}{2}\left(Z_{1}-Z_{345}\right), \quad \text { for } i=3,4, \\
& \lambda_{5}=Z_{5} \pm \frac{1}{2}\left(Z_{1}-Z_{345}\right), \quad \tilde{\lambda}_{345}=Z_{1}+\frac{1}{2}\left(Z_{1}-Z_{345}\right), \\
& \lambda_{6}=\lambda_{7}=0,
\end{aligned}
$$

where $Z_{345} \equiv Z_{3}+Z_{4} \pm Z_{5} \quad$ and $\quad \tilde{\lambda}_{345} \equiv \lambda_{3}+\lambda_{4} \pm \lambda_{5}$. Assuming that $Z_{1} \neq Z_{345}$ [otherwise, Eq. (10.18) is satisfied and we return to the previous case], it follows that $\lambda_{1} \neq \tilde{\lambda}_{345}$. That is, the $\mathbb{Z}_{2} \otimes \Pi_{2}$ symmetry of the quartic terms of the scalar potential of the $\Phi$-basis is manifestly realized. Soft breaking of the $\mathbb{Z}_{2} \otimes \Pi_{2}$ symmetry due to $m_{12}^{2} \neq 0$, where $m_{12}^{2}$ is real (pure imaginary) if $\sin \xi=0(\cos \xi=0)$, is allowed subject to the condition $m_{11}^{2}=m_{22}^{2}$.

If we now impose the custodial symmetry condition, $Z_{4}= \pm Z_{5}$, then it follows that two parameter relations are possible, 
TABLE XII. Classification of 2HDM scalar potentials that possess an unbroken custodial symmetry and satisfy the inert conditions, $Y_{3}=Z_{6}=Z_{7}=0$, thereby exhibiting exact Higgs alignment. The Higgs basis field $\mathcal{H}_{2}$ has been rephased such that $Z_{5}$ is real. In the symmetry limit, the scalar Lagrangian symmetry that is manifestly realized in the $\Phi$-basis is shown along with the corresponding maximal symmetry group (that includes the custodial symmetry in the limit of $g^{\prime} \rightarrow 0$ ) according to the classification provided in Ref. [87]. Excluding the first two lines of the table, all entries correspond to the ERPS4 regime. The corresponding ERPS symmetry may be softly broken if $m_{11}^{2} \neq m_{22}^{2}$ and/or $m_{12}^{2} \neq 0$ as indicated in Table XI. Since GCP3 is equivalent to U(1) $\otimes \Pi_{2}$ when expressed in a different scalar field basis, there is a one-to-one mapping between their corresponding entries above that is consistent with the results of Sec. VIII.

\begin{tabular}{|c|c|c|c|c|}
\hline $\begin{array}{l}\text { Higgs basis conditions } \\
\text { (all cases satisfy } \\
Y_{3}=Z_{6}=Z_{7}=0 \text { ) }\end{array}$ & $\begin{array}{c}\text { Custodial symmetry } \\
\text { conditions }\end{array}$ & $\begin{array}{c}\text { Additional real } \\
\Phi \text {-basis constraints }\end{array}$ & $\begin{array}{l}\text { Scalar Lagrangian } \\
\text { symmetry }\end{array}$ & $\begin{array}{c}\text { Maximal } \\
\text { symmetry group }\end{array}$ \\
\hline & $Z_{4}= \pm Z_{5} \neq 0$ & $s_{2 \beta}=0$ & $\mathbb{Z}_{2}$ & $\mathbb{Z}_{2} \otimes \mathrm{O}(3)$ \\
\hline & $Z_{4}=Z_{5}=0$ & $s_{2 \beta}=0$ & $\mathrm{U}(1)$ & $\mathrm{SO}(4)$ \\
\hline$Z_{1}=Z_{2} \neq Z_{345}$ & $Z_{4}= \pm Z_{5} \neq 0$ & $c_{2 \beta} \sin 2 \xi=0, \lambda=\lambda_{3}$ or $\lambda_{4}= \pm \lambda_{5}$ & $\mathbb{Z}_{2} \otimes \Pi_{2}$ & $\mathbb{Z}_{2} \otimes \mathbb{Z}_{2} \otimes \mathrm{SO}(3)$ \\
\hline$Z_{1}=Z_{2} \neq Z_{345}$ & $Z_{4}= \pm Z_{5} \neq 0$ & $s_{2 \beta}=0, \lambda_{4}= \pm \lambda_{5}$ & $\mathbb{Z}_{2} \otimes \Pi_{2}$ & $\mathbb{Z}_{2} \otimes \mathbb{Z}_{2} \otimes \mathrm{SO}(3)$ \\
\hline$Z_{1}=Z_{2}=Z_{3}+2 Z_{4}$ & $Z_{4}= \pm Z_{5} \neq 0$ & $c_{2 \beta}=0, \lambda=\lambda_{3}, \lambda_{4} \neq 0$ & $\mathrm{U}(1) \otimes \Pi_{2}$ & $\mathrm{O}(2) \otimes \mathrm{O}(3)$ \\
\hline$Z_{1}=Z_{2}=Z_{3}$ & $Z_{4}= \pm Z_{5} \neq 0$ & $c_{2 \beta}=0, \lambda \neq \lambda_{3}, \lambda_{4}=0$ & $\mathrm{U}(1) \otimes \Pi_{2}$ & $\mathbb{Z}_{2} \otimes \mathrm{O}(4)$ \\
\hline$Z_{1}=Z_{2} \neq Z_{3}$ & $Z_{4}=Z_{5}=0$ & $s_{2 \beta}=0, \lambda \neq \lambda_{3}, \lambda_{4}=0$ & $\mathrm{U}(1) \otimes \Pi_{2}$ & $\mathbb{Z}_{2} \otimes \mathrm{O}(4)$ \\
\hline$Z_{1}=Z_{2}=Z_{3}+2 Z_{4}$ & $Z_{4}=Z_{5} \neq 0$ & $s_{2 \beta^{\prime}} \sin \xi^{\prime}=0, \lambda_{4}^{\prime}=\lambda_{5}^{\prime} \neq 0$ & GCP3 & $\mathrm{O}(2) \otimes \mathrm{O}(3)$ \\
\hline$Z_{1}=Z_{2}=Z_{3}$ & $Z_{4}=-Z_{5} \neq 0$ & $s_{2 \beta^{\prime}} \sin \xi^{\prime}=0, \lambda_{4}^{\prime}=-\lambda_{5}^{\prime} \neq 0$ & GCP3 & $\mathbb{Z}_{2} \otimes \mathrm{O}(4)$ \\
\hline$Z_{1}=Z_{2} \neq Z_{3}$ & $Z_{4}=Z_{5}=0$ & $c_{2 \beta^{\prime}}=\cos \xi^{\prime}=0, \lambda_{4}^{\prime}=-\lambda_{5}^{\prime} \neq 0$ & GCP3 & $\mathbb{Z}_{2} \otimes \mathrm{O}(4)$ \\
\hline$Z_{1}=Z_{2}=Z_{3}$ & $Z_{4}=Z_{5}=0$ & $\lambda_{4}=\lambda_{5}=0$ & $\mathrm{SO}(3)$ & $\mathrm{SO}(5)$ \\
\hline
\end{tabular}

$$
\lambda_{1}=\lambda_{2} \neq \tilde{\lambda}_{345}, \quad \text { where } \lambda_{4}= \pm \lambda_{5}
$$

or

$$
\lambda_{1}=\lambda_{2}=\lambda_{3}, \quad \text { where } \lambda_{4} \neq \pm \lambda_{5} .
$$

Equations (10.24) and (10.25) are related by a change of scalar field basis. For example, if $\sin \xi=0$ then we replace the \pm sign with a plus sign in the above expressions. Starting from Eq. (10.25) and employing Eq. (8.1) to transform $\Phi \rightarrow \bar{\Phi}=U \Phi$, the scalar potential parameters in the new basis satisfy $\bar{m}_{11}^{2}=\bar{m}_{22}^{2}, \bar{m}_{12}^{2} \neq 0$ and

$$
\begin{array}{ll}
\bar{\lambda}_{1}=\bar{\lambda}_{2}=\lambda_{3}+\frac{1}{2}\left(\lambda_{4}-\lambda_{5}\right), & \bar{\lambda}_{3}=\lambda_{3}-\frac{1}{2}\left(\lambda_{4}-\lambda_{5}\right), \\
\bar{\lambda}_{4}=\bar{\lambda}_{5}=\frac{1}{2}\left(\lambda_{4}+\lambda_{5}\right) . &
\end{array}
$$

Since $\bar{\lambda}_{3}+\bar{\lambda}_{4}+\bar{\lambda}_{5}-\bar{\lambda}_{1}=2 \lambda_{5}$, Eq. (10.24) is satisfied in the $\bar{\Phi}$-basis, assuming that $\lambda_{5} \neq 0$. However, if $\lambda_{5}=0$, then $\bar{\lambda}_{1}=\bar{\lambda}_{2}=\bar{\lambda}_{3}+\bar{\lambda}_{4}+\bar{\lambda}_{5}$ is satisfied, corresponding to a softly broken GCP3-symmetric scalar potential in the $\bar{\Phi}$-basis. The case of $\cos \xi=0$ can be similarly treated by rephasing $\Phi_{2} \rightarrow i \Phi_{2}$, and yields a softly broken GCP3'-symmetric scalar potential.

Finally, if $s_{2 \beta}=0$, then one can impose the unbroken or softly broken symmetries of the ERPS4 and the custodial symmetry condition directly in the Higgs basis. In Table XII, we provide a complete classification of the 2HDM scalar potentials that possess an unbroken custodial symmetry and exhibit exact Higgs alignment. For convenience, we have included entries corresponding to both $\mathrm{U}(1) \otimes \Pi_{2}$ and GCP3, which correspond to physically equivalent points in the ERPS4 in light of the results of Sec. VIII.

It is noteworthy that two maximal symmetry groups are associated with both $\mathrm{U}(1) \otimes \Pi_{2}$ and GCP3. These two cases are distinguished by the corresponding Higgs basis conditions. Indeed, one can check that $\mathrm{O}(2) \otimes \mathrm{O}(3)$ is physically distinguished from $\mathbb{Z}_{2} \otimes \mathrm{O}(4)$. In particular, in the cases of softly broken or unbroken $\mathrm{U}(1) \otimes \Pi_{2}$ and GCP3-symmetric scalar potentials, $\mathrm{O}(2) \otimes \mathrm{O}(3)$ is associated with the mass relation, $m_{H^{ \pm}}=m_{H} \neq m_{A}$. In contrast, $\mathbb{Z}_{2} \otimes \mathrm{O}(4)$ is associated with the mass relation $m_{H^{ \pm}}=m_{A}$, which includes the possibility of $m_{H^{ \pm}}=m_{A}=$ $m_{H}$ if in addition $Z_{4}=Z_{5}=0$. The latter is an example of the more general result that any custodial symmetric $2 \mathrm{HDM}$ scalar potential with $Z_{4}=Z_{5}=Z_{6}=Z_{7}=0$ exhibits a Peccei-Quinn $U(1)$ symmetry that is unbroken by the scalar potential and vacuum and thus possesses a scalar spectrum where $H^{ \pm}$is degenerate in mass with both $H$ and $A$.

\section{CONCLUSIONS AND FUTURE DIRECTIONS}

There is a fascinating region of parameter space of the $2 \mathrm{HDM}$ that can be implemented by imposing the generalized $C P$ symmetry, GCP2, on the quartic terms of the scalar potential, which enforces the relations, $\lambda_{1}=\lambda_{2}$ and 
$\lambda_{7}=-\lambda_{6}$. We call this region the ERPS4, generalizing the exceptional region of the parameter space (ERPS) of the 2HDM introduced in Refs. [29,41], where the GCP2 symmetry is also respected by the quadratic terms of the scalar potential and yields the additional constraints, $m_{11}^{2}=$ $m_{22}^{2}$ and $m_{12}^{2}=0$. That is, the ERPS4 is the parameter space of a softly broken GCP2-symmetric scalar potential. In this paper, we have provided a comprehensive study of the many interesting properties of this 2HDM parameter regime, including limiting cases of the ERPS4 parameters that extend the unbroken or softly broken symmetries of the scalar potential beyond GCP2.

We have enumerated the basis invariant conditions that characterize the softly broken GCP2 symmetries and their extensions and evaluated the scalar squared masses and neutral scalar mixing matrices in each of these cases. We have discussed intricacies that arise when scalar potentials originating from two different symmetry conditions yield physically equivalent results. In such cases, although the parameter constraints imposed by the symmetry conditions may differ, one can show that a unitary transformation relates the scalar field bases in which each of the symmetries is manifestly realized. Indeed, a GCP2-symmetric scalar potential is related by a unitary basis transformation to a scalar potential that is invariant with respect to $\mathbb{Z}_{2} \otimes \Pi_{2}$, and a GCP3-symmetric scalar potential is related by a unitary basis transformation to a scalar potential that is invariant with respect to $\mathrm{U}(1) \otimes \Pi_{2}$. These considerations persist even if the corresponding symmetries are softly broken.

The equivalence of the softly broken GCP 2 and $\mathbb{Z}_{2} \otimes$ $\Pi_{2}$ symmetries, as demonstrated below Eq. (4.20), does not yield a simple analytic formula that relates the parameters of the GCP2 basis and the $\mathbb{Z}_{2} \otimes \Pi_{2}$ basis. In this work, our analysis of the ERPS4 always starts from the $\mathbb{Z}_{2} \otimes \Pi_{2}$ basis, from which all subsequent special cases can be analyzed. In contrast, the translation between the GCP3 basis and the $\mathrm{U}(1) \otimes \Pi_{2}$ basis can be made explicit, and a translation between the parameters defined in each of the two basis choices has been provided in Sec. VIII. The results for the softly broken SO(3)symmetric scalar potential, which are different limiting cases of the GCP3 and $\mathrm{U}(1) \otimes \Pi_{2}$ basis choices, ultimately yield identical results given that the form of the softly broken $\mathrm{SO}(3)$-symmetric scalar potential is invariant with respect to an arbitrary unitary transformation of the scalar field basis.

In examining the $C P$-invariance properties of scalar potentials in the ERPS4, we encountered an interesting feature that runs contrary to a statement usually found in the literature. In a softly broken $\mathbb{Z}_{2} \otimes \Pi_{2}$-symmetric scalar potential where the magnitudes of the two neutral scalar field vevs are equal, we originally noticed in Ref. [37] that the scalar potential and vacuum were both $C P$ conserving even though the relative phase between the potentially complex parameters $m_{12}^{2}$ and $\lambda_{5}$ could not be removed by separate rephasings of the scalar fields $\Phi_{1}$ and $\Phi_{2}$. In contrast, outside of the ERPS4 regime, it is straightforward to show that if $\lambda_{6}=\lambda_{7}=0$ and $\operatorname{Im}\left(\lambda_{5}^{*}\left[m_{12}^{2}\right]^{2}\right) \neq 0$, then the corresponding scalar potential is explicitly $C P$ violating. We were able to identify an alternative definition of $C P$, denoted by $\mathrm{GCP}^{\prime}$ in Tables $\mathrm{V}$ and VI, which provides an explanation for why the softly broken $\mathbb{Z}_{2} \otimes \Pi_{2}$-symmetric scalar potential with $v_{1}=v_{2}$ always preserves a $C P$ symmetry.

Perhaps even more astonishing was that in a softly broken GCP3-symmetric scalar potential with $\operatorname{Im}\left(\lambda_{5}^{*}\left[m_{12}^{2}\right]^{2}\right) \neq 0$, the scalar potential and vacuum are always $C P$ invariant independently of the vevs. In this case, the identification of the relevant $C P$ transformation law is more obscure (see Appendix C). Of course, this result becomes almost trivial by transforming to the $\mathrm{U}(1) \otimes \Pi_{2}$ basis, where a simple rephasing can be performed to remove all potential complex phases from the corresponding scalar potential parameters. Moreover, in both $C P$-conserving examples above where $\operatorname{Im}\left(\lambda_{5}^{*}\left[m_{12}^{2}\right]^{2}\right) \neq 0$, a more general unitary transformation of the scalar fields exists that can transform directly to a real scalar field basis in which the $C P$ invariance of the scalar potential is manifest. Because the scalar potentials of the ERPS4 are quite constrained, such a unitary transformation is still consistent with the parameter constraints imposed by the softly broken $\mathbb{Z}_{2} \otimes \Pi_{2}$ and GCP3 symmetries.

A very important subset of the ERPS4 is the so-called inert limit where the Higgs basis parameters satisfy $Y_{3}=Z_{6}=Z_{7}=0$. In this parameter regime Higgs alignment is exact, which means that there exists a neutral scalar whose tree-level properties coincide with those of the SM Higgs boson. Indeed, the LHC Higgs data have already confirmed at the 10\%-20\% level that the properties of the observed Higgs boson (of mass $125 \mathrm{GeV}$ ) are consistent with the predictions of the Standard Model. Consequently, any phenomenologically viable extended Higgs sector must exhibit at least an approximate Higgs alignment. One can achieve an approximate Higgs alignment automatically in the decoupling limit where the masses of all additional scalars are significantly larger than $125 \mathrm{GeV}$. However, it is of interest to consider the possibility of approximate Higgs alignment without decoupling, as this scenario would provide more options for potential discoveries of new scalars of an extended Higgs sector in future LHC runs. Higgs alignment without decoupling can be achieved without a fine-tuning of scalar sector parameters if a symmetry is present that can enforce the Higgs alignment. Thus, in the 2HDM it is especially useful to provide a complete classification of all such symmetries. The simplest example of a $2 \mathrm{HDM}$ with this property is the IDM which possesses an unbroken $\mathbb{Z}_{2}$ or U(1) symmetry in the Higgs basis. All other 2HDM scenarios that provide a natural explanation for exact 
Higgs alignment lie in the ERPS4 regime. The complete classification has been provided in Table XI.

A phenomenologically viable extended Higgs sector must also be consistent with precision electroweak constraints. The observation that the electroweak $\rho$-parameter is approximately equal to one strongly suggests that the scalar potential should be invariant under a custodial symmetry. The ERPS4 enters in these considerations as well, since one of the two ways to satisfy the requirements of custodial symmetry is provided by the inert limit. Thus, combining the requirements of exact Higgs alignment and custodial symmetry yields a classification of 2HDM scenarios that is exhibited in Table XII.

Finally, there is one aspect of the 2HDM that has been almost completely ignored in our comprehensive study of the ERPS4-namely, the Higgs-fermion Yukawa interactions. There is a reason for this neglect. In a $2 \mathrm{HDM}$ with one generation of quarks and leptons, it is not possible to construct a Yukawa Lagrangian that respects a GCP2 symmetry or any of its symmetry extensions. If three generations of quarks and leptons are present, then it is possible to construct a set of Yukawa interactions that respect a GCP2 or GCP3 symmetry by positing transformation laws that involve fermions of different generations. However, all such constructions are inconsistent with the constraints of experimental observations with the possible exception of one very special implementation of the GCP3 symmetry in Ref. [89]. It is not clear whether these remarks also hold if one were to construct a Yukawa Lagrangian that respects a $\mathbb{Z}_{2} \otimes \Pi_{2}$ or $\mathrm{U}(1) \otimes \Pi_{2}$ symmetry. Scalar Lagrangians that are constrained by symmetries that are physically the same when only the scalar sector is considered might be different once fermions are included. This possibility is presently under study.

If there is no phenomenologically successful $2 \mathrm{HDM}$ Yukawa Lagrangian consistent with the ERPS4 regime, then there are two possible approaches. In one approach advocated in Refs. [39,90], the ERPS4 conditions are imposed at the Planck scale. The Yukawa interactions represent a hard breaking of the symmetries responsible for the ERPS4 regime. Hence, renormalization group evolution down to the electroweak scale will generate an effective 2HDM that deviates from the ERPS4 but might retain some of its best features (e.g., approximate Higgs alignment and approximate custodial symmetry). The second approach follows the proposals of Refs. [70,91], where vectorlike quark and lepton partners are introduced in an extended Yukawa Lagrangian. In this case, one can construct a Yukawa Lagrangian that is consistent with the ERPS4 regime (even in a one generation model of fermions and the vectorlike partners). To ensure that the vectorlike fermions are sufficiently heavy to avoid current LHC search limits, one can introduce explicit mass terms for the vectorlike fermions, which then generate the soft-breaking squared mass terms of the ERPS4.
In either of these two approaches, one can determine parameter regimes that are consistent with observed Higgs boson phenomena, while setting useful targets for precision Higgs studies at the LHC and future Higgs factories now under development. It is also of theoretical interest to seek out ultraviolet complete models that include the Yukawa sector to ultimately explain the origin of the fundamental symmetries that underlie the approximate symmetries governing the 2HDM at the electroweak scale. ${ }^{36}$ We shall defer such matters to future studies. Given that the ERPS4 provides a simple framework for the scalar potential of an extended Higgs sector with a reduced number of free parameters, we would anticipate that useful correlations could emerge, such as relations among various three-scalar couplings, if deviations from SM Higgs properties are detected and/or new scalar states are discovered.

\section{ACKNOWLEDGMENTS}

H. E. H. is supported in part by the U.S. Department of Energy Grant No. DE-SC0010107. H. E. H. is grateful for the hospitality and support during his visit to the Instituto Superior Técnico, Universidade de Lisboa, and he also acknowledges fruitful discussions that took place at the University of Warsaw during visits supported by the HARMONIA project of the National Science Centre, Poland, under Contract No. UMO-2015/18/M/ST2/00518 (2016-2021). The work of J.P. S. is supported in part by the Portuguese Fundação para a Ciência e Tecnologia (FCT) under Contracts No. CERN/FIS-PAR/0008/2019, No. PTDC/FIS-PAR/29436/2017, No. UIDB/00777/2020, and No. UIDP/00777/2020.

\section{APPENDIX A: THE POSSIBILITY OF SPONTANEOUS $C P$ VIOLATION}

Consider the case of an explicitly $C P$-conserving, softly broken $\mathbb{Z}_{2}$-symmetric scalar potential written in a real scalar field basis, where $\lambda_{6}=\lambda_{7}=0$ and the two potentially complex scalar potential parameters, $m_{12}^{2}$ and $\lambda_{5}$, are real and nonzero. In this case, spontaneous $C P$ violation is possible $[68,98,99] .{ }^{37}$ It is instructive to examine the minimum and stability conditions under the assumption that $\left\langle\Phi_{1}^{0}\right\rangle=v_{1} / \sqrt{2}$ and $\left\langle\Phi_{2}^{0}\right\rangle=v_{2} e^{i \xi} / \sqrt{2}$, where $v_{1}$ and $v_{2}$ are real and positive. Following the analysis of Appendix B of Ref. [42], the vacuum value of the scalar potential is

\footnotetext{
${ }^{36}$ For example, attempts to construct ultraviolet complete models that yield Higgs alignment without decoupling in the 2HDM can be found in Refs. [92-97].

${ }^{37}$ Spontaneous $C P$ violation is also possible if $m_{12}^{2}$ is purely imaginary and $\lambda_{5}$ is real. In this case, one can redefine $\Phi_{2} \rightarrow i \Phi_{2}$, which renders $m_{12}^{2}$ real while transforming $\lambda_{5} \rightarrow-\lambda_{5}$ and $\xi \rightarrow \xi+\frac{1}{2} \pi$.
} 


$$
\begin{aligned}
V_{\text {vac }}= & \frac{1}{2} m_{11}^{2} v_{1}^{2}+\frac{1}{2} m_{22}^{2} v_{2}^{2}-m_{12}^{2} v_{1} v_{2} \cos \xi+\frac{1}{8}\left(\lambda_{1} v_{1}^{4}+\lambda_{2} v_{2}^{4}\right) \\
& +\frac{1}{4}\left(\lambda_{3}+\lambda_{4}-\lambda_{5}\right) v_{1}^{2} v_{2}^{2}+\frac{1}{2} \lambda_{5} v_{1}^{2} v_{2}^{2} \cos ^{2} \xi .
\end{aligned}
$$

The scalar potential minimum conditions are

$$
\begin{gathered}
0=\frac{\partial V_{\mathrm{vac}}}{\partial v_{1}}=m_{11}^{2} v_{1}-m_{12}^{2} v_{2} \cos \xi+\frac{1}{2} \lambda_{1} v_{1}^{3}+\frac{1}{2} \lambda_{345} v_{1} v_{2}^{2} \\
0=\frac{\partial V_{\mathrm{vac}}}{\partial v_{2}}=m_{22}^{2} v_{2}-m_{12}^{2} v_{1} \cos \xi+\frac{1}{2} \lambda_{2} v_{2}^{3}+\frac{1}{2} \lambda_{345} v_{1}^{2} v_{2} \\
0=\frac{1}{v} \frac{\partial V_{\mathrm{vac}}}{\partial \xi}=\frac{v_{1} v_{2}}{v}\left(m_{12}^{2}-\lambda_{5} v_{1} v_{2} \cos \xi\right) \sin \xi
\end{gathered}
$$

where $\lambda_{345}$ is defined below Eq. (2.7). The vacuum is $C P$ conserving if $\sin 2 \xi=0[98,100],{ }^{38}$ whereas the vacuum is potentially $C P$ violating if $\sin 2 \xi \neq 0$.

First consider the case of $\sin 2 \xi=0$. Having excluded $m_{12}^{2}=0$ from consideration (cf. footnote 38), it follows that $\sin \xi=0$. Without loss of generality, we may take $\cos \xi=1$ by rephasing $\Phi_{2} \rightarrow-\Phi_{2}$ (which also changes the

$$
H=\left(\begin{array}{c}
m_{12}^{2} \frac{v_{2}}{v_{1}}+\lambda_{1} v_{1}^{2} \\
-m_{12}^{2}+\left(\lambda_{3}+\lambda_{4}+\lambda_{5}\right) v_{1} v_{2}
\end{array}\right.
$$

Stability requires that $\operatorname{Tr} H>0$ and $\operatorname{det} H>0$. In addition, we demand that the squared masses of the neutral Higgs bosons should be positive. Using the results of Ref. [42], the following quantities all must be positive:

$$
\begin{gathered}
m_{A}^{2}=\left(\frac{m_{12}^{2}}{v_{1} v_{2}}-\lambda_{5}\right) v^{2}, \\
m_{h}^{2}+m_{H}^{2}=m_{A}^{2}+\lambda_{1} v_{1}^{2}+\lambda_{2} v_{2}^{2}, \\
m_{h}^{2} m_{H}^{2}=\frac{m_{A}^{2}}{v^{2}}\left[\lambda_{1} v_{1}^{4}+\lambda_{2} v_{2}^{4}+2\left(\lambda_{3}+\lambda_{4}\right) v_{1}^{2} v_{2}^{2}\right] \\
+\left[\lambda_{1} \lambda_{2}-\left(\lambda_{3}+\lambda_{4}\right)^{2}\right] v_{1}^{2} v_{2}^{2} .
\end{gathered}
$$

Note that the trace and determinant of the Hessian matrix are related to the squared masses of the neutral scalars,

\footnotetext{
${ }^{38}$ If $\cos \xi=0$ then Eq. (A4) yields $m_{12}^{2}=0$. In this case the $\mathbb{Z}_{2}$ symmetry of the scalar potential is explicitly preserved and spontaneous $C P$ violation does not occur [98] (see also Theorem 23.3 of Ref. [68]).
}

sign of $m_{12}^{2}$ but otherwise has no effect on the other scalar potential parameters). Then, Eqs. (A2) and (A3) yield

$$
\begin{aligned}
& m_{11}^{2}=m_{12}^{2} \frac{v_{2}}{v_{1}}-\frac{1}{2} \lambda_{1} v_{1}^{2}-\frac{1}{2}\left(\lambda_{3}+\lambda_{4}+\lambda_{5}\right) v_{2}^{2}, \\
& m_{22}^{2}=m_{12}^{2} \frac{v_{1}}{v_{2}}-\frac{1}{2} \lambda_{2} v_{2}^{2}-\frac{1}{2}\left(\lambda_{3}+\lambda_{4}+\lambda_{5}\right) v_{1}^{2} .
\end{aligned}
$$

The stability conditions can be discerned from the Hessian. Computing the relevant second derivatives,

$$
\begin{aligned}
\frac{\partial^{2} V_{\mathrm{vac}}}{\partial v_{1}^{2}} & =m_{11}^{2}+\frac{3}{2} \lambda_{1} v_{1}^{2}+\frac{1}{2}\left(\lambda_{3}+\lambda_{4}+\lambda_{5}\right) v_{2}^{2} \\
& =m_{12}^{2} \frac{v_{2}}{v_{1}}+\lambda_{1} v_{1}^{2}, \\
\frac{\partial^{2} V_{\mathrm{vac}}}{\partial v_{2}^{2}} & =m_{22}^{2}+\frac{3}{2} \lambda_{2} v_{2}^{2}+\frac{1}{2}\left(\lambda_{3}+\lambda_{4}+\lambda_{5}\right) v_{1}^{2} \\
& =m_{12}^{2} \frac{v_{1}}{v_{2}}+\lambda_{2} v_{2}^{2}, \\
\frac{\partial^{2} V_{\mathrm{vac}}}{\partial v_{1} \partial v_{2}} & =-m_{12}^{2}+\left(\lambda_{3}+\lambda_{4}+\lambda_{5}\right) v_{1} v_{2},
\end{aligned}
$$

after applying the results of Eqs. (A5) and (A6). Thus, the Hessian matrix is given by

$$
\left.\begin{array}{c}
-m_{12}^{2}+\left(\lambda_{3}+\lambda_{4}+\lambda_{5}\right) v_{1} v_{2} \\
m_{12}^{2} \frac{v_{1}}{v_{2}}+\lambda_{2} v_{2}^{2}
\end{array}\right) .
$$

$$
\operatorname{Tr} H=m_{h}^{2}+m_{H}^{2}+\lambda_{5} v^{2},
$$

$\operatorname{det} H=m_{h}^{2} m_{H}^{2}+\lambda_{5}\left[\left(\lambda_{5}+\frac{2 m_{A}^{2}}{v^{2}}\right) v_{1}^{2} v_{2}^{2}+\lambda_{1} v_{1}^{4}+\lambda_{2} v_{2}^{4}\right]$.

Next, consider the case of $\sin 2 \xi \neq 0$. In this case, it is convenient to replace Eq. (A4) with

$$
0=\frac{1}{v} \frac{\partial V_{\text {vac }}}{\partial \cos \xi}=\frac{v_{1} v_{2}}{v}\left(-m_{12}^{2}+\lambda_{5} v_{1} v_{2} \cos \xi\right),
$$

which yields $m_{12}^{2}=\lambda_{5} v_{1} v_{2} \cos \xi$. Inserting this result into Eqs. (A2) and (A3), it follows that

$$
\begin{aligned}
& m_{11}^{2}=-\frac{1}{2} \lambda_{1} v_{1}^{2}-\frac{1}{2}\left(\lambda_{3}+\lambda_{4}-\lambda_{5}\right) v_{2}^{2}, \\
& m_{22}^{2}=-\frac{1}{2} \lambda_{2} v_{2}^{2}-\frac{1}{2}\left(\lambda_{3}+\lambda_{4}-\lambda_{5}\right) v_{1}^{2} .
\end{aligned}
$$

The elements of the $3 \times 3$ Hessian matrix are given by the following second derivatives: 


$$
\begin{aligned}
\frac{\partial^{2} V_{\mathrm{vac}}}{\partial v_{1}^{2}} & =m_{11}^{2}+\frac{3}{2} \lambda_{1} v_{1}^{2}+\frac{1}{2}\left(\lambda_{3}+\lambda_{4}+\lambda_{5} \cos 2 \xi\right) v_{2}^{2} \\
& =\lambda_{1} v_{1}^{2}+\lambda_{5} v_{2}^{2} \cos ^{2} \xi \\
\frac{\partial^{2} V_{\mathrm{vac}}}{\partial v_{2}^{2}} & =m_{22}^{2}+\frac{3}{2} \lambda_{2} v_{2}^{2}+\frac{1}{2}\left(\lambda_{3}+\lambda_{4}+\lambda_{5} \cos 2 \xi\right) v_{1}^{2} \\
& =\lambda_{2} v_{2}^{2}+\lambda_{5} v_{1}^{2} \cos ^{2} \xi \\
\frac{\partial^{2} V_{\mathrm{vac}}}{\partial v_{1} \partial v_{2}} & =-m_{12}^{2} \cos \xi+\left(\lambda_{3}+\lambda_{4}+\lambda_{5} \cos 2 \xi\right) v_{1} v_{2} \\
& =\left(\lambda_{3}+\lambda_{4}-\lambda_{5} \sin ^{2} \xi\right) v_{1} v_{2},
\end{aligned}
$$

$$
H=v^{2}\left(\begin{array}{ccc}
\lambda_{1} c_{\beta}^{2}+\lambda_{5} s_{\beta}^{2} \cos ^{2} \xi & \left(\lambda_{3}+\lambda_{4}-\lambda_{5} \sin ^{2} \xi\right) s_{\beta} c_{\beta} & \lambda_{5} v^{2} s_{\beta}^{2} c_{\beta} \cos \xi \\
\left(\lambda_{3}+\lambda_{4}-\lambda_{5} \sin ^{2} \xi\right) s_{\beta} c_{\beta} & \lambda_{2} s_{\beta}^{2}+\lambda_{5} c_{\beta}^{2} \cos ^{2} \xi & \lambda_{5} s_{\beta} c_{\beta}^{2} \cos \xi \\
\lambda_{5} s_{\beta}^{2} c_{\beta} \cos \xi & \lambda_{5} s_{\beta} c_{\beta}^{2} \cos \xi & \lambda_{5} s_{\beta}^{2} c_{\beta}^{2}
\end{array}\right)
$$

$$
\begin{gathered}
\frac{1}{v^{2}} \frac{\partial^{2} V_{\mathrm{vac}}}{\partial(\cos \xi)^{2}}=\frac{\lambda_{5} v_{1}^{2} v_{2}^{2}}{v^{2}}, \\
\frac{1}{v} \frac{\partial^{2} V_{\mathrm{vac}}}{\partial v_{1} \partial \cos \xi}=\frac{\lambda_{5} v_{1} v_{2}^{2} \cos \xi}{v}, \\
\frac{1}{v} \frac{\partial^{2} V_{\mathrm{vac}}}{\partial v_{2} \partial \cos \xi}=\frac{\lambda_{5} v_{1}^{2} v_{2} \cos \xi}{v} .
\end{gathered}
$$

Thus, the Hessian matrix is given by where $s_{\beta} \equiv v_{2} / v$ and $c_{\beta} \equiv v_{1} / v$. Stability requires that $H$ is positive definite. By Sylvester's criterion [101], it follows that the principal minors must all be positive. A necessary (although not sufficient) condition is that all diagonal elements of $H$ must be positive. In light of Eq. (2.2), we conclude that $\lambda_{5}>0$ is a necessary condition for spontaneous $C P$ violation.

\section{APPENDIX B: AN INVARIANT CHARACTERIZATION OF THE ERPS4 AND CONSEQUENCES FOR CP SYMMETRY}

In Eq. (4.20), we provided an invariant characterization of the ERPS4 that is defined by $\lambda_{1}=\lambda_{2}$ and $\lambda_{7}=-\lambda_{6}$, which if realized in one scalar field basis is then satisfied in all scalar field bases. Using Eq. (4.9) and employing the identify,

$$
\sigma_{a b}^{B} \sigma_{c d}^{B}=2 \delta_{a d} \delta_{b c}-\delta_{a b} \delta_{c d},
$$

it follows that the ERPS4 invariant can be rewritten in terms of the quartic coefficients of the scalar potential,

$\mathcal{Z}=\frac{1}{8} \operatorname{Tr}\left(\left[Z^{(1)}+Z^{(2)}\right]^{2}\right)-\frac{1}{16}\left(\operatorname{Tr} Z^{(1)}+\operatorname{Tr} Z^{(2)}\right)^{2}$,

where, following Ref. [29], we have defined

$$
\begin{aligned}
& Z_{a d}^{(1)} \equiv \delta_{b c} Z_{a b, c d}=Z_{a b, b d}=\left(\begin{array}{ll}
\lambda_{1}+\lambda_{4} & \lambda_{6}+\lambda_{7} \\
\lambda_{6}^{*}+\lambda_{7}^{*} & \lambda_{2}+\lambda_{4}
\end{array}\right), \\
& Z_{b d}^{(2)} \equiv \delta_{a c} Z_{a b, c d}=Z_{a b, a d}=\left(\begin{array}{ll}
\lambda_{1}+\lambda_{3} & \lambda_{6}+\lambda_{7} \\
\lambda_{6}^{*}+\lambda_{7}^{*} & \lambda_{2}+\lambda_{3}
\end{array}\right),
\end{aligned}
$$

and the $Z_{a b, c d}$ are defined in terms of the quartic couplings of the scalar potential in Eq. (C4). One can simplify Eq. (B2) using the symmetry properties of the $Z_{a b, c d}$ to obtain a slightly more compact form than was originally obtained in Ref. [29],

$$
\begin{aligned}
\mathcal{Z} & \equiv \frac{1}{2} \operatorname{Tr}\left\{\left(Z^{(1)}\right)^{2}\right\}-\frac{1}{4}\left[\operatorname{Tr} Z^{(1)}\right]^{2} \\
& =\frac{1}{4}\left(\lambda_{1}-\lambda_{2}\right)^{2}+\left|\lambda_{6}+\lambda_{7}\right|^{2} .
\end{aligned}
$$

As noted below Eq. (4.20), the ERPS4 corresponds to the invariant condition, $\mathcal{Z}=0$, which implies that $\lambda_{1}=\lambda_{2}$ and $\lambda_{7}=-\lambda_{6}$. This condition is invariant with respect to changes in the scalar field basis, $\Phi_{a} \rightarrow U_{a \bar{b}} \Phi_{b}$ for any $U \in \mathrm{U}(2)$. Thus, if $\lambda_{1}=\lambda_{2}$ and $\lambda_{7}=-\lambda_{6}$ in one basis, then the same relation holds in any scalar field basis. In particular, it holds in the Higgs basis, which implies that $Z_{1}=Z_{2}$ and $Z_{7}=-Z_{6}$.

One can make an even stronger statement that for any scalar potential of the ERPS4, there exists a scalar field basis where $\lambda_{6}=\lambda_{7}=0$ and $\operatorname{Im} \lambda_{5}=0$ (the latter after an appropriate rephasing of $\Phi_{2}$ ), which defines the $\mathbb{Z}_{2} \otimes \Pi_{2}$ basis of Sec. V. A simple proof of this statement was provided below Eq. (4.20). Moreover, if $C P$ is conserved then the scalar field basis in which $\lambda_{6}=\lambda_{7}=0$ can be explicitly identified, as shown in Appendix B of Ref. [37] and summarized below.

The first step is to go to the Higgs basis of the ERPS4 where $Z_{1}=Z_{2}$ and $Z_{7}=-Z_{6}$. If $Z_{6}=0$ then it trivially follows that $\lambda_{6}=\lambda_{7}=0$ in the Higgs basis. If $Z_{6} \neq 0$, then consider a $\mathrm{U}(2)$ transformation from the Higgs basis to the $\Phi$-basis with neutral vevs $v_{1} / \sqrt{2}$ and $v_{2} e^{i \xi} / \sqrt{2}$ where $v_{1}$ and $v_{2}$ are positive, $\tan \beta \equiv v_{2} / v_{1}$, and $0 \leq \xi<2 \pi$. It is 
straightforward to derive a $\Phi$-basis expression for $\lambda_{6}=-\lambda_{7}$ in terms of Higgs basis parameters,

$$
\begin{aligned}
\lambda_{6} e^{i \xi}= & \frac{1}{2} s_{2 \beta} c_{2 \beta}\left[Z_{1}-Z_{34}-\operatorname{Re}\left(Z_{5} e^{2 i \xi}\right)\right]-\frac{1}{2} i s_{2 \beta} \operatorname{Im}\left(Z_{5} e^{2 i \xi}\right) \\
& +c_{4 \beta} \operatorname{Re}\left(Z_{6} e^{i \xi}\right)+i c_{2 \beta} \operatorname{Im}\left(Z_{6} e^{i \xi}\right),
\end{aligned}
$$

where $Z_{34} \equiv Z_{3}+Z_{4}$. We now search for values of $\beta$ and $\xi$ such that $\lambda_{6}=0$. Moreover, since $\lambda_{7}=-\lambda_{6}$ in the ERPS4, it follows that if $(\beta, \xi)$ yield $\lambda_{6}=0$ then so does $\left(\frac{1}{2} \pi-\beta, \xi+\pi\right)$, corresponding to the interchange of the scalar doublet fields, $\Phi_{1} \leftrightarrow \Phi_{2}$.

Since $Z_{6} \neq 0$, we may write $Z_{6}=\left|Z_{6}\right| e^{i \theta_{6}}$. It is then convenient to define

$$
\bar{\xi} \equiv \xi+\theta_{6}
$$

where $\bar{\xi}$ is defined modulo $\pi$. In the case where $C P$ is preserved by the scalar potential and vacuum, $\operatorname{Im}\left(Z_{5}^{*} Z_{6}^{2}\right)=0$, as noted above Eq. (5.47). ${ }^{39}$ Inserting $e^{i \xi}=$ $e^{i \xi^{\prime}} Z_{6}^{*} /\left|Z_{6}\right|$ and $\operatorname{Im}\left(Z_{5}^{*} Z_{6}^{2}\right)=0$ into Eq. (B6), we search for values of $\beta$ and $\xi$ such that

$$
\begin{aligned}
& s_{2 \beta} \operatorname{Re}\left(Z_{5}^{*} Z_{6}^{2}\right) \sin 2 \bar{\xi}=2 c_{2 \beta}\left|Z_{6}\right|^{3} \sin \bar{\xi}, \\
& s_{2 \beta} c_{2 \beta}\left[\left|Z_{6}\right|^{2}\left(Z_{1}-Z_{34}\right)-\operatorname{Re}\left(Z_{5}^{*} Z_{6}^{2}\right) \cos 2 \bar{\xi}\right] \\
& =-2 c_{4 \beta}\left|Z_{6}\right|^{3} \cos \bar{\xi} .
\end{aligned}
$$

We can immediately obtain one solution to Eq. (B8), $\sin \bar{\xi}=0$ or equivalently $\cos \bar{\xi}= \pm 1$. The twofold ambiguity was anticipated in the remarks below Eq. (B6). Inserting $\cos \bar{\xi}= \pm 1$ into Eq. (B9) yields a quadratic equation for $\cot 2 \beta$,

$2\left|Z_{6}\right| \cot ^{2} 2 \beta \pm\left(Z_{1}-Z_{34}-\frac{\operatorname{Re}\left(Z_{5}^{*} Z_{6}^{2}\right)}{\left|Z_{6}\right|^{2}}\right) \cot 2 \beta-2\left|Z_{6}\right|=0$,

which possesses two real roots whose product is equal to -1 . As a result, one ends up with four choices of $(\beta, \xi)$, where $0 \leq \beta \leq \frac{1}{2} \pi$ and $\cos \bar{\xi}= \pm 1$, for which Eqs. (B8) and (B9) are satisfied.

Moreover, additional solutions can be found if $\sin \bar{\xi} \neq 0$, in which case one can divide Eq. (B8) by $\sin \bar{\xi}$. Solving Eq. (B8) for $c_{2 \beta} / s_{2 \beta}$ and inserting this result into Eq. (B9) yields

\footnotetext{
${ }^{39}$ If $C P$ is violated, then $\operatorname{Im}\left(Z_{5}^{*} Z_{6}^{2}\right) \neq 0$ and the existence of the scalar field basis where $\lambda_{6}=\lambda_{7}=0$ can be identified numerically, although no simple analytic expressions exist for $\beta$ and $\xi[37,102]$.
}

$$
\begin{aligned}
& \cos \bar{\xi}\left\{\left[\operatorname{Re}\left(Z_{5}^{*} Z_{6}^{2}\right)\right]^{2}+\operatorname{Re}\left(Z_{5}^{*} Z_{6}^{2}\right)\left|Z_{6}\right|^{2}\left(Z_{1}-Z_{34}\right)-2\left|Z_{6}\right|^{6}\right\} \\
& \quad=0 .
\end{aligned}
$$

One immediate solution to this equation is $\cos \bar{\xi}=0$, which we can then plug back into Eq. (B8) to obtain $\cos 2 \beta=0$. Thus, we learn that $\left(\beta=\frac{1}{4} \pi, \bar{\xi}=\frac{1}{2} \pi\right)$ and $\left(\beta=\frac{1}{4} \pi, \bar{\xi}=\frac{3}{2} \pi\right)$ are also solutions to Eqs. (B8) and (B9).

The above results are consistent with the result of Sec. V. Equation (5.19) provides a relation between $Z_{6}$ and the scalar potential parameters of the $\mathbb{Z}_{2} \otimes \Pi_{2}$ basis, which is of the form $Z_{6}=(x+i y) e^{-i \xi}$. Thus, $x+i y=\left|Z_{6}\right| e^{i \bar{\xi}}$ and we can identify

$$
\tan \bar{\xi}=\frac{y}{x}=-\frac{\lambda_{5} \sin 2 \xi}{\left[\lambda(1-R)+2 \lambda_{5} \sin ^{2} \xi\right] c_{2 \beta}} .
$$

Taking into account the values of $(\beta, \bar{\xi})$ obtained above that provide solutions to Eqs. (B8) and (B9), we see that $\sin \bar{\xi}=0$ corresponds to $\sin 2 \xi=0$ and $\cos \bar{\xi}=0$ corresponds to $c_{2 \beta}=0$. Since this analysis was based on the assumption that $\operatorname{Im}\left(Z_{5}^{*} Z_{6}^{2}\right)=0$, we have reproduced the result of Eq. (5.48).

It is now instructive to compute $\operatorname{Im}\left(\lambda_{5}^{*}\left[m_{12}^{2}\right]^{2}\right)$ in the $\mathbb{Z}_{2} \otimes \Pi_{2}$ basis. Using Eq. (B11) of Ref. [37], written in a slightly different form under the assumption that $c_{4 \beta} \neq 0$, we obtain ${ }^{40}$

$$
\begin{aligned}
\operatorname{Im}\left(\lambda_{5}^{*}\left[m_{12}^{2}\right]^{2}\right)= & -\frac{\left|Z_{6}\right| v^{4} s_{2 \beta} \sin \bar{\xi}}{8 c_{4 \beta}}\left\{c_{4 \beta}\left(\frac{2 Y_{2}}{v^{2}}\right)^{2}\right. \\
& -\frac{2 Y_{2}}{v^{2}}\left[s_{2 \beta}^{2} Z_{1}+\left(1-3 c_{2 \beta}^{2}\right) Z_{34}\right]-c_{2 \beta}^{2} \mathcal{R}_{5}^{2} \\
& +\left(Z_{1}+\frac{2 Y_{2}}{v^{2}}\right) \mathcal{R}_{5}+Z_{34}\left(Z_{34} c_{2 \beta}^{2}-Z_{1} s_{2 \beta}^{2}\right) \\
& \left.-\frac{4 c_{4 \beta}}{s_{2 \beta}^{2}}\left|Z_{6}\right|^{2} \sin ^{2} \bar{\xi}\right\},
\end{aligned}
$$

where

$$
\begin{aligned}
\mathcal{R}_{5} & \equiv \operatorname{Re}\left(Z_{5} e^{2 i \xi}\right) \\
& =\frac{\operatorname{Re}\left(Z_{5}^{*} Z_{6}^{2}\right) \cos 2 \bar{\xi}+\operatorname{Im}\left(Z_{5}^{*} Z_{6}^{2}\right) \sin 2 \bar{\xi}}{\left|Z_{6}\right|^{2}} .
\end{aligned}
$$

Under the assumption that $\operatorname{Im}\left(Z_{5}^{*} Z_{6}^{2}\right)=0$, the results obtained above imply that either $\sin \bar{\xi}=0$ or $\cos \bar{\xi}=0$. If $\sin \bar{\xi}=0$ then it immediately follows that $\operatorname{Im}\left(\lambda_{5}^{*}\left[m_{12}^{2}\right]^{2}\right)=0$. That is, one can rephase the scalar doublet fields in the GCP2 basis to obtain a scalar potential whose coefficients are all real in a scalar field basis where the vevs are also real.

\footnotetext{
${ }^{40}$ If $c_{4 \beta}=0$, then the expression given in Eq. (B11) of Ref. [37] is more useful.
} 
In contrast, if $\cos \bar{\xi}=0$, which implies that $c_{2 \beta}=0$ as noted below Eq. (B11), then Eq. (B13) yields

$$
\begin{aligned}
\operatorname{Im}\left(\lambda_{5}^{*}\left[m_{12}^{2}\right]^{2}\right)= & \pm \frac{v^{4}\left|Z_{6}\right|}{8}\left\{4\left|Z_{6}\right|^{2}-\left(Z_{1}+\frac{2 Y_{2}}{v^{2}}\right)^{2}\right. \\
& \left.+\left(Z_{1}+\frac{2 Y_{2}}{v^{2}}\right)\left(Z_{1}-Z_{34}-\frac{\operatorname{Re}\left(Z_{5}^{*} Z_{6}^{2}\right)}{\left|Z_{6}\right|^{2}}\right)\right\},
\end{aligned}
$$

which is generically nonzero. Thus, it follows that if $\beta=\frac{1}{4} \pi$ and $\operatorname{Im} m_{12}^{2} \neq 0$ in the GCP2 basis, then one cannot remove all complex phases from the scalar potential with a simple rephasing of the Higgs doublet fields. Nevertheless, the scalar potential and vacuum are $C P$ conserving since $\operatorname{Im}\left(Z_{5}^{*} Z_{6}^{2}\right)=0$ implies that a real Higgs basis exists (i.e., all Higgs basis scalar potential parameters are real after an appropriate rephasing of the Higgs basis field $\mathcal{H}_{2}$ ).

In the analysis presented above, we used Eq. (B11) to conclude that $\cos \bar{\xi}=0$. However, there is an alternative solution to Eq. (B11) where the coefficient of $\cos \bar{\xi}$ vanishes. Indeed, this alternative solution corresponds to the case of the softly broken GCP3-symmetric 2HDM where Eq. (6.13) is satisfied. In this case, division of Eq. (B8) by $\sin \bar{\xi}$ is permitted when $\sin \bar{\xi} \neq 0$, which yields

$$
\cos \bar{\xi}=\frac{\left|Z_{6}\right|^{3} \cot 2 \beta}{\operatorname{Re}\left(Z_{5}^{*} Z_{6}^{2}\right)} .
$$

Plugging this result into Eq. (B13) yields $\operatorname{Im}\left(\lambda_{5}^{\prime}\left[m_{12}^{\prime 2}\right]^{2}\right) \neq 0$ for generic values of the scalar potential parameters and $\beta$. In particular, in the GCP3 basis (where the scalar potential parameters are designated with prime superscripts), there exists a residual $C P$ invariance despite the fact that $\operatorname{Im}\left(\lambda_{5}^{\prime}\left[m_{12}^{\prime 2}\right]^{2}\right) \neq 0$ when $\sin \bar{\xi}^{\prime} \neq 0$, independently of the value of $\beta^{\prime}$. In contrast, $\lambda_{5}=0$ and $Z_{6}=\left|Z_{6}\right| e^{i \theta_{6}}=$ $\pm\left|Z_{6}\right| e^{-i \xi}$ [cf. Eq. (6.11)] in the $\mathrm{U}(1) \otimes \Pi_{2}$ basis, in which case Eq. (B7) yields $\sin \bar{\xi}=0$.

\section{APPENDIX C: $C P$ INVARIANCE OF THE SOFTLY BROKEN GCP3-SYMMETRIC SCALAR POTENTIAL}

The softly broken GCP3 scalar potential contains a complex parameter, $m_{12}^{\prime 2}$, in a basis in which the only other potentially complex parameter, $\lambda_{5}^{\prime}$, is taken to be real. Moreover, there is a relative phase between the two vevs. Thus, naively one would conjecture that the scalar sector of the softly broken GCP3-symmetric 2HDM is $C P$ violating. However, we have demonstrated that by changing the scalar field basis, this scalar potential can be transformed into a softly broken $\mathrm{U}(1) \otimes \Pi_{2}$ scalar potential in which $\lambda_{5}=0$. Then, one can rephase either
$\Phi_{1}$ or $\Phi_{2}$ to remove the phase of $m_{12}^{2}$, which yields an explicitly $C P$-conserving scalar potential. Moreover, one can show that the scalar potential minimum condition in the explicitly $\mathrm{CP}$-conserving basis yields two vevs with no relative complex phase. Hence, it follows that the softly broken GCP3-symmetric $2 \mathrm{HDM}$ is explicitly $C P$ conserving, and the vacuum also preserves the $C P$ symmetry. These observations imply that in the original GCP3 basis, one should be able to identify a residual generalized $C P$ transformation under which the GCP3 scalar potential and vacuum are invariant. The purpose of this Appendix is to provide the explicit construction of this generalized $C P$ transformation.

We begin by rewriting Eq. (2.1) following the notation of Ref. [29],

$$
\mathcal{V}(\Phi)=Y_{a \bar{b}}\left(\Phi_{\bar{a}}^{\dagger} \Phi_{b}\right)+\frac{1}{2} Z_{a \bar{b} \bar{c} \bar{d}}\left(\Phi_{\bar{a}}^{\dagger} \Phi_{b}\right)\left(\Phi_{\bar{c}}^{\dagger} \Phi_{d}\right),
$$

where the indices $a, \bar{b}, c$ and $\bar{d}$ can take one of two values 1, 2 (with an implicit sum over barred and unbarred index pairs of the same letter), and Hermiticity and symmetry under the interchange of barred and unbarred indices imply that

$$
Y_{a \bar{b}}=\left(Y_{b \bar{a}}\right)^{*}, \quad Z_{a \bar{b} c \bar{d}} \equiv Z_{c \bar{d} a \bar{b}}=\left(Z_{b \bar{a} d \bar{c}}\right)^{*} .
$$

Note that as a matrix,

$$
Y=\left(\begin{array}{ll}
Y_{11} & Y_{12} \\
Y_{12}^{*} & Y_{22}
\end{array}\right)=\left(\begin{array}{cc}
m_{11}^{2} & -m_{12}^{2} \\
-\left(m_{12}^{2}\right)^{*} & m_{22}^{2}
\end{array}\right),
$$

where the minus sign in the definition of $m_{12}^{2}$ is conventional. It is convenient to assemble the elements of the tensor $Z_{a b c d}$ into a $4 \times 4$ Hermitian matrix (denoted by $Z$ ) as follows. First, we introduce a slightly different notation for the components of $Z$,

$$
Z_{a c, b d} \equiv Z_{a \bar{b} c \bar{d}},
$$

where the first pair of indices of $Z_{a c, b d}$ consists of unbarred indices and the second pair consists of barred indices. In this notation, it is conventional to omit the bars in the second pair of indices. ${ }^{41}$ With this notation, each element of a row of the matrix $Z$ is denoted by a pair of subscripts. These index pairs arranged in the order 11, 12, 21 and 22, and similarly for the pair of subscripts denoting each element of a column of $Z$. The matrix $Z$ is then given by

\footnotetext{
${ }^{41}$ The reader is cautioned that in contrast to Eq. (C4), the symbol $Z_{a b, c d}$ employed in Ref. [41] is equivalent to $Z_{a \bar{b} c \bar{d}}$ without an interchange of the indices $b$ and $c$.
} 


$$
\begin{aligned}
Z & =\left(\begin{array}{lll}
Z_{11,11} & Z_{11,12} & Z_{11,21} Z_{11,22} \\
Z_{12,11} & Z_{12,12} & Z_{12,21} Z_{12,22} \\
Z_{21,11} & Z_{21,12} & Z_{21,21} Z_{21,22} \\
Z_{22,11} & Z_{22,12} & Z_{22,21} Z_{22,22}
\end{array}\right) \\
& =\left(\begin{array}{llll}
\lambda_{1} & \lambda_{6} & \lambda_{6} & \lambda_{5} \\
\lambda_{6}^{*} & \lambda_{3} & \lambda_{4} & \lambda_{7} \\
\lambda_{6}^{*} & \lambda_{4} & \lambda_{3} & \lambda_{7} \\
\lambda_{5}^{*} & \lambda_{7}^{*} & \lambda_{7}^{*} & \lambda_{2}
\end{array}\right) .
\end{aligned}
$$

Under a basis transformation,

$$
\Phi_{a} \rightarrow \Phi_{a}^{\prime}=U_{a \bar{b}} \Phi_{b}, \quad \Phi_{\bar{a}}^{\dagger} \rightarrow \Phi_{\bar{a}}^{\prime \dagger}=\Phi_{\bar{b}}^{\dagger} U_{b \bar{a}}^{\dagger},
$$

where $U \in \mathrm{U}(2)$ is a $2 \times 2$ unitary matrix $\left(U_{b \bar{a}}^{\dagger} U_{a \bar{c}}=\delta_{b \bar{c}}\right)$. Under this unitary basis transformation, the vevs are transformed as $\left\langle\Phi_{a}^{0}\right\rangle \rightarrow\left\langle\Phi_{a}^{\prime 0}\right\rangle=U_{a \bar{b}}\left\langle\Phi_{b}^{0}\right\rangle$. Moreover, the gauge covariant kinetic terms of the scalar fields are invariant under a unitary basis transformation, whereas the coefficients $Y_{a \bar{b}}$ and $Z_{a b, c d}$ transform covariantly with respect to $\mathrm{U}(2)$ transformations as

$$
\begin{aligned}
Y_{a \bar{b}} \rightarrow Y_{a \bar{b}}^{\prime} & =U_{a \bar{b}} Y_{c \bar{d}} U_{d \bar{b}}^{\dagger}=\left(U Y U^{\dagger}\right)_{a \bar{b}}, \\
Z_{a b, c d} \rightarrow Z_{a b, c d}^{\prime} & =U_{a \bar{e}} U_{b \bar{g}} Z_{e g, f h} U_{f \bar{c}}^{\dagger} U_{h \bar{d}}^{\dagger} \\
& =\left[(U \otimes U) Z\left(U^{\dagger} \otimes U^{\dagger}\right)\right]_{a b, c d},
\end{aligned}
$$

where the Kronecker product of two $2 \times 2$ matrices is a $4 \times 4$ matrix given in block matrix form by

$$
A \otimes B=\left(\begin{array}{ll}
A_{11} B & A_{12} B \\
A_{21} B & A_{22} B
\end{array}\right) .
$$

The Kronecker product of two matrices satisfies the following properties [103]:

$$
\begin{gathered}
(A \otimes B)(C \otimes D)=A C \otimes B D, \\
(A \otimes B)^{\dagger}=A^{\dagger} \otimes B^{\dagger}, \\
(A \otimes B)^{\top}=A^{\top} \otimes B^{\top}, \\
(A \otimes B)^{-1}=A^{-1} \otimes B^{-1}, \text { if } A^{-1} \text { and } B^{-1} \text { exist. }
\end{gathered}
$$

In particular, if $A$ and $B$ are unitary then so is $A \otimes B$. The Kronecker product $A \otimes B$ can be represented by a rank four tensor whose components are given by

$$
(A \otimes B)_{a b, c d} \equiv A_{a c} B_{b d},
$$

where a row of the matrix $A \otimes B$ is denoted by a pair of subscripts that are arranged in the order 11, 12, 21 and 22 (and similarly for the pair of subscripts denoting the columns of $A \otimes B)$. This convention yields the $4 \times 4$ matrix representation of $A \otimes B$ given in Eq. (C9). Hence, it follows that

$$
\begin{aligned}
U_{a \bar{e}} U_{b \bar{g}} Z_{e g, f h} U_{f \bar{c}}^{\dagger} U_{h \bar{d}}^{\dagger} & =(U \otimes U)_{a b, e g} Z_{e g, f h}\left(U^{\dagger} \otimes U^{\dagger}\right)_{f h, c d} \\
& =\left[(U \otimes U) Z\left(U^{\dagger} \otimes U^{\dagger}\right)\right]_{a b, c d},
\end{aligned}
$$

as indicated in Eq. (C8). That is, Eqs. (C7) and (C8) are equivalent to the matrix equations,

$$
Y^{\prime}=U Y U^{\dagger}, \quad Z^{\prime}=(U \otimes U) Z\left(U^{\dagger} \otimes U^{\dagger}\right) .
$$

It is common to consider the standard $C P$ transformation of the scalar fields as

$$
\Phi_{a}(t ; \overrightarrow{\boldsymbol{x}}) \rightarrow \Phi_{a}^{\mathrm{CP}}(t ; \overrightarrow{\boldsymbol{x}})=\Phi_{a}^{*}(t ;-\overrightarrow{\boldsymbol{x}}),
$$

where we shall no longer distinguish between barred and unbarred indices, and the reference to the time $(t)$ and space $(\overrightarrow{\boldsymbol{x}})$ coordinates will henceforth be suppressed. However, in the presence of several scalars with the same quantum numbers, $\mathrm{U}(2)$ basis transformations can be included in the definition of the $C P$ transformation. This yields the generalized $C P$ transformation (GCP) $[68,104],{ }^{42}$

$$
\begin{gathered}
\Phi_{a}^{\mathrm{GCP}}=e^{i \gamma} X_{a b} \Phi_{b}^{*} \equiv e^{i \gamma} X_{a b}\left(\Phi_{b}^{\dagger}\right)^{\top}, \\
\Phi_{a}^{\dagger \mathrm{GCP}}=e^{-i \gamma} X_{a b}^{*} \Phi_{b}^{\top} \equiv e^{-i \gamma} X_{a b}^{*}\left(\Phi_{b}^{\dagger}\right)^{*},
\end{gathered}
$$

where $X$ is an arbitrary unitary matrix of unit determinant and $\gamma \in \mathbb{R}$. We will indicate below Eq. (C25) how the complex phase factor $e^{i \gamma}$ is determined.

Note that the transformation $\Phi_{a} \rightarrow \Phi_{a}^{\mathrm{GCP}}$, where $\Phi_{a}^{\mathrm{GCP}}$ is given by Eq. (C18), leaves invariant the gauge covariant kinetic terms of the scalar fields. The GCP transformation of a scalar field bilinear yields

$$
\Phi_{a}^{\dagger \mathrm{GCP}} \Phi_{b}^{\mathrm{GCP}}=X_{a c}^{*} X_{b d}\left(\Phi_{c} \Phi_{d}^{\dagger}\right)^{\top},
$$

which does not depend on the complex phase factor $e^{i \gamma}$. Under this GCP transformation, the quadratic terms of the potential may be written as

\footnotetext{
${ }^{42}$ For early work on generalized $C P$ transformations, see Refs. [105-107]. Generalized $C P$ transformations in the context of the 2HDM have been treated in Refs. [30,41,58,59,67,87,89,108-112].
} 


$$
\begin{aligned}
Y_{a b} \Phi_{a}^{\dagger \mathrm{GCP}} \Phi_{b}^{\mathrm{GCP}} & =Y_{a b} X_{a c}^{*} X_{b d} \Phi_{d}^{\dagger} \Phi_{c} \\
& =X_{b d} Y_{b a}^{*} X_{a c}^{*} \Phi_{d}^{\dagger} \Phi_{c} \\
& =X_{c a} Y_{c d}^{*} X_{d b}^{*} \Phi_{a}^{\dagger} \Phi_{b} \\
& =\left(X^{\dagger} Y X\right)_{a b}^{*} \Phi_{a}^{\dagger} \Phi_{b},
\end{aligned}
$$

after making use of the Hermiticity of $Y$ [cf. Eq. (C2)] and appropriately relabeling the indices. A similar argument can be made for the quartic terms, by employing the properties of the Kronecker product given in Eqs. (C10)(C13). We conclude that the scalar potential is invariant under the GCP transformation exhibited in Eq. (C18) if and only if the scalar potential coefficients obey

$$
\begin{gathered}
Y_{a b}^{*}=X_{c a}^{*} Y_{c d} X_{d b}=\left(X^{\dagger} Y X\right)_{a b}, \\
Z_{a b, c d}^{*}=X_{e a}^{*} X_{g b}^{*} Z_{e g, f h} X_{f c} X_{h d},
\end{gathered}
$$

or equivalently,

$$
Y^{*}=X^{\dagger} Y X, \quad Z^{*}=\left(X^{\dagger} \otimes X^{\dagger}\right) Z(X \otimes X) .
$$

Finally, we must check to see whether the GCP symmetry is preserved by the vacuum, in which case the following condition must be satisfied:

$$
\left\langle\Phi_{a}^{0}\right\rangle=e^{i \gamma} X_{a b}\left\langle\Phi_{b}^{\dagger 0}\right\rangle .
$$

The complex phase factor $e^{i \gamma}$ will be chosen subject to the convention where $\left\langle\Phi_{1}^{0}\right\rangle$ is real and non-negative. The latter can always be arranged by performing an appropriate hypercharge $\mathrm{U}(1)_{\mathrm{Y}}$ transformation on the scalar doublet fields, which has no effect on the coefficients of the scalar potential.

So far, we have assumed that all statements apply in the $\Phi$-basis. If we now perform a basis transformation to the $\Phi^{\prime}$-basis as indicated by Eq. (C6), then we can express the scalar potential in terms of the $\Phi^{\prime}$-basis scalar potential parameters,

$\mathcal{V}\left(\Phi^{\prime}\right)=Y_{a b}^{\prime}\left(\Phi_{a}^{\prime \dagger} \Phi_{b}^{\prime}\right)+\frac{1}{2} Z_{a c, b d}^{\prime}\left(\Phi_{a}^{\prime \dagger} \Phi_{b}^{\prime}\right)\left(\Phi_{c}^{\prime \dagger} \Phi_{d}^{\prime}\right)$,

where $Y_{a b}^{\prime}$ and $Z_{a c, b d}^{\prime}$ are given by Eqs. (C7) and (C8), respectively.

Suppose that $V(\Phi)$ is invariant under the GCP transformation of Eq. (C18) with the matrix X. Equation (C22) guarantees that $Y^{*}=X^{\dagger} Y X$. Now, Eq. (C7) relates the coefficients in the two bases through $Y=U^{\dagger} Y^{\prime} U$. It then follows that

$$
U^{\top} Y^{*} U^{*}=X^{\dagger}\left(U^{\dagger} Y^{\prime} U\right) X
$$

which implies that

$$
Y^{*}=\left(U^{*} X^{\dagger} U^{\dagger}\right) Y^{\prime}\left(U X U^{\top}\right)=X^{\prime \dagger} Y^{\prime} X^{\prime},
$$

where $X^{\prime}=U X U^{\top}$. A similar argument can be made for the quartic terms, by employing the properties of the Kronecker product given in Eqs. (C10)-(C13). Thus, we conclude that $V\left(\Phi^{\prime}\right)$ is invariant under a new GCP transformation with matrix

$$
e^{i \gamma^{\prime}} X^{\prime}=e^{i \gamma^{\prime}} U X U^{\top}
$$

The phase $\gamma^{\prime}$ is not fixed by this computation and must instead be determined by examining Eq. (C25) in the $\Phi^{\prime}$-basis in a convention where $\left\langle\Phi_{1}^{\prime 0}\right\rangle$ is real and nonnegative.

To construct the residual GCP transformation that is a symmetry of the softly broken GCP3-symmetric scalar potential, we begin our analysis in the $\mathrm{U}(1) \otimes \Pi_{2}$ basis. The parameters of the quadratic part of the scalar potential are specified in Eq. (C3), where $Y_{12} \equiv\left|Y_{12}\right| e^{i \theta_{12}}$ is potentially complex. ${ }^{43}$ In addition, the parameters of the quartic part of the scalar potential satisfy [cf. Eq. (C5)]

$$
\begin{aligned}
\lambda & =Z_{11,11}=Z_{22,22}, \quad \lambda_{3}=Z_{12,12}=Z_{21,21}, \\
\lambda_{4} & =Z_{12,21}=Z_{21,12},
\end{aligned}
$$

and all the other $Z_{a b, c d}$ vanish. The softly broken $\mathrm{U}(1) \otimes \Pi_{2}$-symmetric scalar potential is invariant with respect to a GCP transformation with matrix

$$
e^{i \gamma} X=\left(\begin{array}{cc}
1 & 0 \\
0 & e^{-2 i \theta_{12}}
\end{array}\right)
$$

The phase $\gamma=-\theta_{12}$ has been chosen in anticipation of Eq. (C34) below.

To establish the presence of the residual GCP symmetry, we first verify that Eq. (C22) is satisfied,

$$
\begin{aligned}
& \left(\begin{array}{cc}
Y_{11} & \left|Y_{12}\right| e^{-i \theta_{12}} \\
\left|Y_{12}\right| e^{i \theta_{12}} & Y_{22}
\end{array}\right) \\
& =\left(\begin{array}{cc}
1 & 0 \\
0 & e^{2 i \theta_{12}}
\end{array}\right)\left(\begin{array}{cc}
Y_{11} & \left|Y_{12}\right| e^{i \theta_{12}} \\
\left|Y_{12}\right| e^{-i \theta_{12}} & Y_{22}
\end{array}\right)\left(\begin{array}{cc}
1 & 0 \\
0 & e^{-2 i \theta_{12}}
\end{array}\right) .
\end{aligned}
$$

Next, we verify that Eq. (C23) is satisfied:

\footnotetext{
${ }^{43}$ Here, we are assuming that $Y_{12} \neq 0$. In the case of $Y_{12}=0$, one can choose $\gamma=\xi$ and replace $\theta_{12}$ with $-\xi$ in the definition of $X$ given in Eq. (C31) to ensure that Eqs. (C32)-(C34) are all satisfied, thereby establishing invariance of the scalar potential and the vacuum under the residual generalized $C P$ transformation.
} 


$$
\begin{aligned}
\left(\begin{array}{cccc}
\lambda & 0 & 0 & 0 \\
0 & \lambda_{3} & \lambda_{4} & 0 \\
0 & \lambda_{4} & \lambda_{3} & 0 \\
0 & 0 & 0 & \lambda
\end{array}\right) & =\left(\begin{array}{cccc}
1 & 0 & 0 & 0 \\
0 & e^{2 i \theta_{12}} & 0 & 0 \\
0 & 0 & e^{2 i \theta_{12}} & 0 \\
0 & 0 & 0 & e^{4 i \theta_{12}}
\end{array}\right) \\
& \times\left(\begin{array}{cccc}
\lambda & 0 & 0 & 0 \\
0 & \lambda_{3} & \lambda_{4} & 0 \\
0 & \lambda_{4} & \lambda_{3} & 0 \\
0 & 0 & 0 & \lambda
\end{array}\right) \\
& \times\left(\begin{array}{cccc}
1 & 0 & 0 & 0 \\
0 & e^{-2 i \theta_{12}} & 0 & 0 \\
0 & 0 & e^{-2 i \theta_{12}} & 0 \\
0 & 0 & 0 & e^{-4 i \theta_{12}}
\end{array}\right) .
\end{aligned}
$$

Finally, the GCP symmetry is preserved by the vacuum if Eq. (C25) is satisfied. The vevs are given by $v_{a}=$ $\left(v_{1}, v_{2} e^{i \xi}\right)$, where $v_{1}$ and $v_{2}$ are positive and $\xi$ is determined by the scalar potential minimum condition [cf. Eq. (6.4)], $\operatorname{Im}\left(Y_{12} e^{i \xi}\right)=0$, which yields $\sin \left(\theta_{12}+\xi\right)=0$, under the assumption that $Y_{12} \neq 0$ (cf. footnote 43). It then follows that $\theta_{12}+\xi=0 \bmod \pi$, which demonstrates that Eq. (C25) is indeed satisfied. That is,

$$
\left(\begin{array}{c}
v_{1} \\
v_{2} e^{i \xi}
\end{array}\right)=\left(\begin{array}{cc}
1 & 0 \\
0 & e^{-2 i \theta_{12}}
\end{array}\right)\left(\begin{array}{c}
v_{1} \\
v_{2} e^{-i \xi}
\end{array}\right) .
$$

In the GCP 3 basis, $\lambda_{5}^{\prime}=\lambda_{1}^{\prime}-\lambda_{3}^{\prime}-\lambda_{4}^{\prime}$ is real and nonzero. Now, it is not immediately obvious that the softly broken GCP3-symmetric $2 \mathrm{HDM}$ preserves a $C P$ symmetry, since $\operatorname{Im}\left(\lambda_{5}^{*}\left[m_{12}^{2}\right]^{2}\right) \neq 0$, which implies that one cannot rephase the scalar doublet fields to remove the phase of $m_{12}^{2}$. Nevertheless, we know that $C P$ is a symmetry of the softly broken GCP3-symmetric scalar potential and vacuum since it corresponds to a softly broken symmetric $\mathrm{U}(1) \otimes \Pi_{2}$ scalar potential expressed in a different basis. Thus, it should be possible to explicitly construct the residual generalized $C P$ transformation that preserves the softly broken GCP3-symmetric scalar potential and vacuum by employing Eq. (C29), with $U$ given by Eq. (8.1). Indeed, we will construct $X^{\prime}$ below and explicitly verify that Eqs. (C24) and (C25) are satisfied when expressed in terms of the GCP3 basis parameters.

Equations (8.1) and (C29) yield y $^{44}$

$$
e^{i \gamma^{\prime}} X^{\prime}=-i e^{i \gamma^{\prime}}\left(\begin{array}{cc}
-s_{12} & c_{12} \\
c_{12} & s_{12}
\end{array}\right),
$$

\footnotetext{
${ }^{44}$ In obtaining Eq. (C35), we have absorbed the phase $\phi$ [cf. Eq. (8.1)] into the definition of $\gamma^{\prime}$.
}

where $s_{12} \equiv \sin \theta_{12}$ and $c_{12} \equiv \cos \theta_{12}$. Using Eq. (8.49), which we can rewrite as

$$
Y_{12}=\operatorname{Re} Y_{12}^{\prime}+\frac{1}{2} i\left(Y_{22}^{\prime}-Y_{11}^{\prime}\right)
$$

it follows that

$$
\begin{aligned}
& c_{12}=\frac{\operatorname{Re} Y_{12}^{\prime}}{\sqrt{\left(\operatorname{Re} Y_{12}^{\prime}\right)^{2}+\frac{1}{4}\left(Y_{22}^{\prime}-Y_{11}^{\prime}\right)^{2}}}, \\
& s_{12}=\frac{Y_{22}^{\prime}-Y_{11}^{\prime}}{2 \sqrt{\left(\operatorname{Re} Y_{12}^{\prime}\right)^{2}+\frac{1}{4}\left(Y_{22}^{\prime}-Y_{11}^{\prime}\right)^{2}}} .
\end{aligned}
$$

One can check that Eq. (C22) is satisfied in the GCP3 basis by rewriting this equation as

$$
\left(\begin{array}{ll}
Y_{11}^{\prime} & Y_{12}^{\prime *} \\
Y_{12}^{\prime} & Y_{22}^{\prime}
\end{array}\right)-\left(\begin{array}{cc}
-s_{12} & c_{12} \\
c_{12} & s_{12}
\end{array}\right)\left(\begin{array}{ll}
Y_{11}^{\prime} & Y_{12}^{\prime} \\
Y_{12}^{\prime *} & Y_{22}^{\prime}
\end{array}\right)\left(\begin{array}{cc}
-s_{12} & c_{12} \\
c_{12} & s_{12}
\end{array}\right)=0
$$

To verify Eq. (C38), we multiply out the left-hand side above to obtain

$$
\left[2 s_{12} \operatorname{Re} Y_{12}^{\prime}-c_{12}\left(Y_{22}^{\prime}-Y_{11}^{\prime}\right)\right]\left(\begin{array}{cc}
c_{12} & s_{12} \\
s_{12} & -c_{12}
\end{array}\right)=0
$$

which is equal to the zero matrix after making use of Eq. (C37).

Next, we check the validity of Eq. (C23) in the GCP3 basis. The explicit form of $X^{\prime} \otimes X^{\prime}$ is given by

$$
X^{\prime} \otimes X^{\prime}=\left(\begin{array}{cccc}
s_{12}^{2} & -s_{12} c_{12} & -s_{12} c_{12} & c_{12}^{2} \\
-s_{12} c_{12} & -s_{12}^{2} & c_{12}^{2} & s_{12} c_{12} \\
-s_{12} c_{12} & c_{12}^{2} & -s_{12}^{2} & s_{12} c_{12} \\
c_{12}^{2} & s_{12} c_{12} & s_{12} c_{12} & s_{12}^{2}
\end{array}\right) \text {. }
$$

In the GCP3 basis,

$$
Z^{\prime}=\left(\begin{array}{cccc}
\lambda^{\prime} & 0 & 0 & \lambda^{\prime}-\lambda_{3}^{\prime}-\lambda_{4}^{\prime} \\
0 & \lambda_{3}^{\prime} & \lambda_{4}^{\prime} & 0 \\
0 & \lambda_{4}^{\prime} & \lambda_{3}^{\prime} & 0 \\
\lambda^{\prime}-\lambda_{3}^{\prime}-\lambda_{4}^{\prime} & 0 & 0 & \lambda^{\prime}
\end{array}\right)
$$

Indeed, Eq. (C23) is satisfied in the GCP3 basis, independently of the value of $\theta_{12}$.

Our final check involves confirming the validity of Eq. (C25) in the GCP3 basis. This computation will then determine the phase $\gamma^{\prime}$. Before performing the 
computation, we shall record an important result that is a consequence of the scalar potential minimum condition that is used to fix $\xi^{\prime}$. In light of Eqs. (7.5) and (7.6), it follows that ${ }^{45}$

$$
\cos \xi^{\prime}=-\frac{c_{12} c_{2 \beta^{\prime}}}{s_{12} s_{2 \beta^{\prime}}}
$$

after employing Eq. (C37).

Thus, we must verify

$$
\begin{aligned}
\left(\begin{array}{c}
c_{\beta^{\prime}} \\
e^{i \xi^{\prime}} s_{\beta^{\prime}}
\end{array}\right) & =-i e^{i \gamma^{\prime}}\left(\begin{array}{cc}
-s_{12} & c_{12} \\
c_{12} & s_{12}
\end{array}\right)\left(\begin{array}{c}
c_{\beta^{\prime}} \\
e^{-i \xi^{\prime}} s_{\beta^{\prime}}
\end{array}\right) \\
& =\left(\begin{array}{c}
-i e^{i \gamma^{\prime}}\left(-s_{12} c_{\beta^{\prime}}+c_{12} s_{\beta^{\prime}} e^{-i \xi^{\prime}}\right) \\
-i e^{i \gamma^{\prime}}\left(c_{12} c_{\beta^{\prime}}+s_{12} s_{\beta^{\prime}} e^{-i \xi^{\prime}}\right)
\end{array}\right) .
\end{aligned}
$$

We can immediately determine $\gamma^{\prime}$ from the equation for $c_{\beta^{\prime}}$ in Eq. (C43),

$$
-i e^{i \gamma^{\prime}}=-s_{12}+c_{12} e^{i \xi^{\prime}} \tan \beta^{\prime}
$$

One can verify that $-s_{12}+c_{12} e^{i \xi^{\prime}} \tan \beta^{\prime}$ is a complex number of unit modulus after making use of Eq. (C42), which provides one independent check of the validity of Eq. (C43). The explicit form of the residual GCP symmetry in the GCP3 basis has now been fixed.

Finally, we must verify the second complex equation for $e^{i \xi^{\prime}} s_{\beta^{\prime}}$ given in Eq. (C43),

$$
s_{\beta^{\prime}}=-i e^{i\left(\gamma^{\prime}-\xi^{\prime}\right)}\left(c_{12} c_{\beta^{\prime}}+s_{12} s_{\beta^{\prime}} e^{-i \xi^{\prime}}\right) .
$$

Straightforward algebra shows that Eq. (C45) is an identify after making use of Eqs. (C42) and (C44) to eliminate $\cos \xi^{\prime}$ and $e^{i \gamma^{\prime}}$.

Thus, we have verified that the scalar potential and vacuum of the softly broken GCP3-symmetric 2HDM are invariant with respect to a residual GCP transformation with matrix

$$
e^{i \gamma^{\prime}} X^{\prime}=\left(c_{12} e^{i \xi^{\prime}} \tan \beta^{\prime}-s_{12}\right)\left(\begin{array}{cc}
-s_{12} & c_{12} \\
c_{12} & s_{12}
\end{array}\right),
$$

where $s_{12}$ and $c_{12}$ are given by Eq. (C37) and $\beta^{\prime}$ and $\xi^{\prime}$ are determined by the GCP3 scalar potential parameters as indicated in Eqs. (7.5)-(7.7). Note that although the form of

\footnotetext{
${ }^{45}$ Equation (C42) can also be deduced from Eq. (8.56) after making use of $\tan \xi=-\tan \theta_{12}$, which is a consequence of the scalar potential minimum condition, $\sin \left(\theta_{12}+\xi\right)=0$, obtained above Eq. (C34).
}

$e^{i \gamma^{\prime}} X^{\prime}$ depends on the parameters of the softly broken GCP3 scalar potential, our calculation demonstrates that for any choice of the parameters (and in particular for any choice of the parameters that softly breaks the GCP3 symmetry), there exists a residual GCP symmetry characterized by the matrix $e^{i \gamma^{\prime}} X^{\prime}$.

These results are not surprising given that we knew from the beginning that the softly broken GCP3-symmetric scalar potential is equivalent to a softly broken $U(1) \otimes \Pi_{2}$ symmetric scalar potential where the residual $C P$ symmetry transformation law is identified as GCP1, after removing all complex phases from the coefficients of the scalar potential parameters by an appropriate rephasing of the scalar doublet fields. Nevertheless, it is satisfying to explicitly identify the residual GCP symmetry of the softly broken GCP3-symmetric scalar potential independently of the relations between $\mathrm{U}(1) \otimes \Pi_{2}$ basis and the GCP3 basis obtained in Sec. VIII.

\section{APPENDIX D: SCALAR SQUARED MASS MATRICES IN THE $\Phi$-BASIS}

In Secs. VI and VII, the neutral scalar squared mass matrices were evaluated in the Higgs basis. Of course, the same scalar squared masses can be obtained by computing the eigenvalues of the neutral scalar squared mass matrices evaluated in the $\Phi$-basis (under the assumption that $s_{2 \beta} \neq 0$ ). This computation provides a check of the results obtained in Secs. VI and VII.

For example, for a softly broken $\mathrm{U}(1) \otimes \Pi_{2}$-symmetric scalar potential, the calculation of the eigenvalues of the neutral scalar squared-mass matrix is most easily done after rephasing $m_{12}^{2}$ as described below Eq. (6.5). In this case, one obtains $m_{A}^{2}=2 m_{12}^{2} / s_{2 \beta}$ and the squared masses of $h$ and $H$ (with $m_{H} \leq m_{H}$ ) correspond to the eigenvalues of the $2 \times 2$ matrix,

$$
\begin{aligned}
& \mathcal{M}_{H}^{2} \\
& =\left(\begin{array}{cc}
m_{A}^{2} s_{\beta}^{2}+\lambda v^{2} c_{\beta}^{2} & -s_{\beta} c_{\beta}\left[m_{A}^{2}-\left(\lambda_{3}+\lambda_{4}\right) v^{2}\right] \\
-s_{\beta} c_{\beta}\left[m_{A}^{2}-\left(\lambda_{3}+\lambda_{4}\right) v^{2}\right] & m_{A}^{2} c_{\beta}^{2}+\lambda v^{2} s_{\beta}^{2}
\end{array}\right),
\end{aligned}
$$

with respect to the $\left\{\sqrt{2} \operatorname{Re} \Phi_{1}^{0}-v c_{\beta}, \sqrt{2} \operatorname{Re} \Phi_{2}^{0}-v s_{\beta}\right\}$ basis. One can verify that Eqs. (6.21) and (6.22) are satisfied, as these equations are independent of the choice of scalar field basis.

For a softly broken GCP3-symmetric scalar potential (where parameters and fields are denoted with prime superscripts), the computation of the neutral scalar squared-mass matrix in the $\Phi^{\prime}$-basis (where $s_{2 \beta^{\prime}} \neq 0$ ) is more challenging. After employing the GCP3 condition, $\lambda_{3}^{\prime}+\lambda_{4}^{\prime}=\lambda^{\prime}-\lambda_{5}^{\prime}$, the $4 \times 4$ neutral scalar squared-mass matrix is given by 


$$
\mathcal{M}_{N 4}^{2}=\left(\begin{array}{cc}
\frac{s_{\beta^{\prime}}}{c_{\beta^{\prime}}} R^{\prime}+\lambda^{\prime} v^{2} c_{\beta^{\prime}}^{2} & -\cos \xi^{\prime}\left[R^{\prime}-\left(\lambda^{\prime}-\lambda_{5}^{\prime} \sin ^{2} \xi^{\prime}\right) v^{2} s_{\beta^{\prime}} c_{\beta^{\prime}}\right] \\
-\cos \xi^{\prime}\left[R^{\prime}-\left(\lambda^{\prime}-\lambda_{5}^{\prime} \sin ^{2} \xi^{\prime}\right) v^{2} s_{\beta^{\prime}} c_{\beta^{\prime}}\right] & \frac{c_{\beta^{\prime}}}{s_{\beta^{\prime}}} R^{\prime}+\left(\lambda^{\prime} s_{\beta}^{2} \cos ^{2} \xi^{\prime}+\lambda_{5}^{\prime} c_{\beta}^{2} \sin ^{2} \xi^{\prime}\right) v^{2} \\
\frac{1}{2} \lambda_{5}^{\prime} v^{2} s_{\beta^{\prime}}^{2} \sin 2 \xi^{\prime} & \sin \xi^{\prime}\left[R^{\prime}+\lambda_{5}^{\prime} v^{2} s_{\beta^{\prime}} c_{\beta^{\prime}} \sin ^{2} \xi^{\prime}\right] \\
-\sin \xi^{\prime}\left[R^{\prime}-\left(L^{\prime}-\lambda_{5}^{\prime} \cos ^{2} \xi^{\prime}\right) v^{2} s_{\beta^{\prime}} c_{\beta^{\prime}}\right] & \frac{1}{2} \lambda^{\prime} v^{2} s_{\beta^{\prime}}^{2} \sin 2 \xi^{\prime} \\
\frac{1}{2} \lambda_{5}^{\prime} v^{2} s_{\beta^{\prime}}^{2} \sin 2 \xi^{\prime} & -\sin \xi^{\prime}\left[R^{\prime}-\left(L^{\prime}-\lambda_{5}^{\prime} \cos ^{2} \xi^{\prime}\right) v^{2} s_{\beta^{\prime}} c_{\beta^{\prime}}\right] \\
\sin \xi^{\prime}\left[R^{\prime}+\lambda_{5}^{\prime} v^{2} s_{\beta^{\prime}} c_{\beta^{\prime}} \sin ^{2} \xi^{\prime}\right] & \frac{1}{2} \lambda^{\prime} v^{2} s_{\beta^{\prime}}^{2} \sin 2 \xi^{\prime} \\
\frac{s_{\beta^{\prime}}}{c_{\beta^{\prime}}} R^{\prime}-\lambda_{5}^{\prime} v^{2} s_{\beta^{\prime}}^{2} \cos 2 \xi^{\prime} & -\cos \xi^{\prime}\left[R^{\prime}-\lambda_{5}^{\prime} v^{2} s_{\beta^{\prime}} c_{\beta^{\prime}} \cos ^{2} \xi^{\prime}\right] \\
-\cos \xi^{\prime}\left[R^{\prime}-\lambda_{5}^{\prime} v^{2} s_{\beta^{\prime}} c_{\beta^{\prime}} \cos ^{2} \xi^{\prime}\right] & \frac{c_{\beta^{\prime}}}{s_{\beta^{\prime}}} R^{\prime}+\left(\lambda^{\prime} s_{\beta^{\prime}}^{2} \sin ^{2} \xi^{\prime}-\lambda_{5}^{\prime} c_{\beta^{\prime}}^{2} \cos ^{2} \xi^{\prime}\right) v^{2}
\end{array}\right),
$$

with respect to the $\left\{\sqrt{2} \operatorname{Re} \Phi_{1}^{\prime 0}-v c_{\beta^{\prime}}, \sqrt{2} \operatorname{Re} \Phi_{2}^{\prime 0}-v s_{\beta^{\prime}} \cos \xi^{\prime}, \sqrt{2} \operatorname{Im} \Phi_{1}^{\prime 0}, \sqrt{2} \operatorname{Im} \Phi_{2}^{\prime 0}-v s_{\beta^{\prime}} \sin \xi^{\prime}\right\}$ basis,

where

$$
R^{\prime} \equiv \operatorname{Re}\left(m_{12}^{\prime 2} e^{i \xi^{\prime}}\right), \quad L^{\prime} \equiv \lambda^{\prime}-2 \lambda_{5}^{\prime} \sin ^{2} \xi^{\prime}
$$

The next step is to identify the neutral Goldstone boson, which resides in the Higgs basis field $\mathcal{H}_{1}$, and corresponds to the eigenvector of $\mathcal{M}_{N 4}^{2}$ with zero eigenvalue,

$$
\begin{aligned}
\frac{1}{\sqrt{2}} G^{0} & =\operatorname{Im} \mathcal{H}_{1}^{0} \\
& =-s_{\beta^{\prime}} \sin \xi^{\prime} \operatorname{Re} \Phi_{2}^{\prime 0}+c_{\beta^{\prime}} \operatorname{Im} \Phi_{1}^{\prime 0}+s_{\beta^{\prime}} \cos \xi^{\prime} \operatorname{Im} \Phi_{2}^{\prime 0} .
\end{aligned}
$$

Defining the real orthogonal matrix,

$$
\mathcal{R}=\left(\begin{array}{cccc}
1 & 0 & 0 & 0 \\
0 & \cos \xi^{\prime} & 0 & \sin \xi^{\prime} \\
0 & c_{\beta^{\prime}} \sin \xi^{\prime} & s_{\beta^{\prime}} & -c_{\beta^{\prime}} \cos \xi^{\prime} \\
0 & -s_{\beta^{\prime}} \sin \xi^{\prime} & c_{\beta^{\prime}} & s_{\beta^{\prime}} \cos \xi^{\prime}
\end{array}\right)
$$

one then finds that $\mathcal{R} \mathcal{M}_{N 4}^{2} \mathcal{R}^{\top}$ is a matrix with respect to the rotated basis whose fourth row and column consists entirely of zeros (due to the Goldstone boson). Removing the fourth row and fourth column yields a $3 \times 3$ squaredmass matrix,

$$
\mathcal{M}_{N 3}^{2}=\left(\begin{array}{ccc}
\frac{s_{\beta^{\prime}}}{c_{\beta^{\prime}}} R^{\prime}+\lambda^{\prime} v^{2} c_{\beta^{\prime}}^{2} & -R^{\prime}+L^{\prime} v^{2} s_{\beta^{\prime}} c_{\beta^{\prime}} & \frac{1}{2} \lambda_{5}^{\prime} v^{2} s_{\beta^{\prime}} \sin 2 \xi^{\prime} \\
-R^{\prime}+L^{\prime} v^{2} s_{\beta^{\prime}} c_{\beta^{\prime}} & \frac{c_{\beta^{\prime}}}{s_{\beta^{\prime}}} R^{\prime}+\lambda^{\prime} v^{2} s_{\beta}^{2} & \frac{1}{2} \lambda_{5}^{\prime} v^{2} c_{\beta^{\prime}} \sin 2 \xi^{\prime} \\
\frac{1}{2} \lambda_{5}^{\prime} v^{2} s_{\beta^{\prime}} \sin 2 \xi^{\prime} & \frac{1}{2} \lambda_{5}^{\prime} v^{2} c_{\beta^{\prime}} \sin 2 \xi^{\prime} & \frac{R^{\prime}}{s_{\beta^{\prime}} c_{\beta^{\prime}}}-\lambda_{5}^{\prime} v^{2} \cos 2 \xi^{\prime}
\end{array}\right),
$$

with respect to the $\left\{\sqrt{2} \operatorname{Re} \Phi_{1}^{\prime 0}-v c_{\beta^{\prime}}, \sqrt{2} \operatorname{Re}\left(e^{-i \xi^{\prime}} \Phi_{2}^{\prime 0}\right)-v s_{\beta^{\prime}}, \sqrt{2}\left[s_{\beta^{\prime}} \operatorname{Im} \Phi_{1}^{\prime 0}-c_{\beta^{\prime}} \operatorname{Im}\left(e^{-i \xi^{\prime}} \Phi_{2}^{\prime 0}\right)\right]\right\}$ basis.

The normalized eigenstate corresponding to $A$ is given by

$$
A=\frac{1}{\sqrt{1-s_{2 \beta^{\prime}}^{2} \sin ^{2} \xi^{\prime}}}\left(\begin{array}{c}
s_{\beta^{\prime}} \cos \xi^{\prime} \\
-c_{\beta^{\prime}} \cos \xi^{\prime} \\
-c_{2 \beta^{\prime}} \sin \xi^{\prime}
\end{array}\right),
$$

with corresponding eigenvalue,

$$
m_{A}^{2}=\frac{\operatorname{Re}\left(m_{12}^{\prime 2} e^{i \xi^{\prime}}\right)}{s_{\beta^{\prime}} c_{\beta^{\prime}}}+\lambda_{5}^{\prime} v^{2} \sin ^{2} \xi^{\prime}
$$

$$
\begin{aligned}
A & =\left(1-s_{2 \beta^{\prime}}^{2} \sin ^{2} \xi^{\prime}\right)^{-1 / 2} \sqrt{2}\left\{s_{\beta^{\prime}}\left(\cos \xi^{\prime} \operatorname{Re} \Phi_{1}^{\prime 0}-c_{2 \beta^{\prime}} \sin \xi^{\prime} \operatorname{Im} \Phi_{1}^{\prime 0}\right)+c_{\beta}\left[c_{2 \beta^{\prime}} \sin \xi^{\prime} \operatorname{Im}\left(e^{-i \xi^{\prime}} \Phi_{2}^{\prime 0}\right)-\cos \xi^{\prime} \operatorname{Re}\left(e^{-i \xi^{\prime}} \Phi_{2}^{\prime 0}\right)\right]\right\} \\
& =\left(1-s_{2 \beta^{\prime}}^{2} \sin ^{2} \xi^{\prime}\right)^{-1 / 2} \sqrt{2} \operatorname{Im}\left[i\left(\cos \xi^{\prime}+i c_{2 \beta^{\prime}} \sin \xi^{\prime}\right)\left(s_{\beta^{\prime}} \Phi_{1}^{\prime 0}-c_{\beta^{\prime}} e^{-i \xi^{\prime}} \Phi_{2}^{\prime 0}\right)\right] \\
& =\sqrt{2} \operatorname{Im}\left[i e^{i \psi}\left(s_{\beta^{\prime}} \Phi_{1}^{\prime 0}-c_{\beta^{\prime}} e^{-i \xi^{\prime}} \Phi_{2}^{\prime 0}\right)\right]=\sqrt{2} \operatorname{Im}\left[e^{i \eta^{\prime}}\left(-s_{\beta^{\prime}} e^{i \xi^{\prime}} \Phi_{1}^{\prime 0}+c_{\beta^{\prime}} \Phi_{2}^{\prime 0}\right)\right] \\
& =\sqrt{2} \operatorname{Im} \mathcal{H}_{2}^{0},
\end{aligned}
$$

after making use of Eqs. (5.7), (7.16) and (8.60). 
One can then identify two other mutually orthogonal normalized vectors orthogonal to $A$,

$$
\begin{aligned}
\sqrt{2} \operatorname{Re} \mathcal{H}_{1}^{0}-v & =\left(\begin{array}{c}
c_{\beta^{\prime}} \\
s_{\beta^{\prime}} \\
0
\end{array}\right) \text { and } \\
\sqrt{2} \operatorname{Re} \mathcal{H}_{2}^{0} & =\frac{1}{\sqrt{1-s_{2 \beta^{\prime}}^{2} \sin ^{2} \xi^{\prime}}}\left(\begin{array}{c}
-s_{\beta^{\prime}} c_{2 \beta^{\prime}} \sin \xi^{\prime} \\
c_{\beta^{\prime}} c_{2 \beta^{\prime}} \sin \xi^{\prime} \\
-\cos \xi^{\prime}
\end{array}\right),
\end{aligned}
$$

where the identification of the vectors above with the Higgs basis fields follows the same procedure that yielded Eq. (D9). Hence, if we construct a $3 \times 3$ real orthogonal matrix $\mathcal{O}$ whose rows are given by the transposes of the column vectors exhibited in Eqs. (D10) and (D7), respectively, then it is straightforward to verify that $\mathcal{O M}_{N 3}^{2} \mathcal{O}^{\top}$ is a block diagonal matrix with respect to the $\left\{\sqrt{2} \operatorname{Re} \mathcal{H}_{1}^{0}-v\right.$, $\left.\sqrt{2} \operatorname{Re} \mathcal{H}_{2}^{0}, \sqrt{2} \operatorname{Im} \mathcal{H}_{2}^{0}\right\}$ basis. The upper $2 \times 2$ block of $\mathcal{O M}_{N 3}^{2} \mathcal{O}^{\top}$ can be identified with the $2 \times 2 \mathrm{CP}$-even neutral scalar squared-mass matrix,

$$
\mathcal{M}_{H}^{2}=\left(\begin{array}{cc}
\left(\lambda^{\prime}-\lambda_{5}^{\prime} s_{2 \beta^{\prime}}^{2} \sin ^{2} \xi^{\prime}\right) v^{2} & -\lambda_{5}^{\prime} v^{2} s_{2 \beta^{\prime}} \sin \xi^{\prime}\left(1-s_{2 \beta^{\prime}}^{2} \sin ^{2} \xi^{\prime}\right)^{1 / 2} \\
-\lambda_{5}^{\prime} v^{2} s_{2 \beta^{\prime}} \sin \xi^{\prime}\left(1-s_{2 \beta^{\prime}}^{2} \sin ^{2} \xi^{\prime}\right)^{1 / 2} & m_{A}^{2}-\lambda_{5}^{\prime} v^{2}\left(1-s_{2 \beta^{\prime}}^{2} \sin ^{2} \xi^{\prime}\right)
\end{array}\right)
$$

which reproduces the result of Eq. (7.23), and the squared mass of the $C P$-odd scalar, $m_{A}^{2}$ [which is given by Eq. (D8)], is the 33 element of $\mathcal{O} \mathcal{M}_{N 3}^{2} \mathcal{O}^{\top}$.

Clearly this is not the preferred method for computing the squared masses of the neutral scalars that derive from a softly broken GCP3-symmetric scalar potential, in light of the much simpler Higgs basis computation given in Sec. VII.

\section{APPENDIX E: THE IDM IN THE Ф-BASIS}

The inert doublet model (IDM) can be defined as a $2 \mathrm{HDM}$ in which the $2 \mathrm{HDM}$ Lagrangian and vacuum are invariant under a $\mathbb{Z}_{2}$ symmetry, $\mathcal{H}_{1} \rightarrow \mathcal{H}_{1}, \mathcal{H}_{2} \rightarrow-\mathcal{H}_{2}$, in the Higgs basis. In particular, $\mathcal{H}_{2}$ is odd under the $\mathbb{Z}_{2}$ symmetry, whereas all other fields of the $2 \mathrm{HDM}$ (i.e., $\mathcal{H}_{1}$, the gauge bosons, and the fermions) are even under the $\mathbb{Z}_{2}$ symmetry. Of course, one is free to transform the scalar field basis from the Higgs basis to an arbitrary $\Phi$-basis by employing the unitary matrix $U$ given in Eq. (9.12).

Suppose one is given a $2 \mathrm{HDM}$ scalar potential in a $\Phi$-basis, where the vevs of the scalar fields yield $\tan \beta=$ $\left|\left\langle\Phi_{2}^{0}\right\rangle /\left\langle\Phi_{1}^{0}\right\rangle\right|$ and $\xi=\arg \left[\left\langle\Phi_{1}^{0}\right\rangle^{*}\left\langle\Phi_{2}^{0}\right\rangle\right]$. What are the conditions on the scalar potential parameters that imply that the model under consideration is the IDM? To answer this question, we start in the Higgs basis with $Y_{3}=Z_{6}=Z_{7}=0$, as mandated by the $\mathbb{Z}_{2}$ symmetry. Employing Eqs. (A18)-(A28) of Ref. [37], it then follows that in the $\Phi$-basis, the scalar potential parameters must satisfy the following conditions:

$$
\begin{gathered}
\operatorname{Im}\left(m_{12}^{2} e^{i \xi}\right)=0, \\
\left(m_{22}^{2}-m_{11}^{2}\right) s_{2 \beta}=2 \operatorname{Re}\left(m_{12}^{2} e^{i \xi}\right) c_{2 \beta},
\end{gathered}
$$

$$
\begin{gathered}
c_{4 \beta} \operatorname{Re}\left[\left(\lambda_{6}-\lambda_{7}\right) e^{i \xi}\right] \\
=\frac{1}{2} s_{2 \beta} c_{2 \beta}\left[\lambda_{1}+\lambda_{2}-2\left(\lambda_{3}+\lambda_{4}+\operatorname{Re}\left(\lambda_{5} e^{2 i \xi}\right)\right)\right], \\
c_{2 \beta} \operatorname{Im}\left[\left(\lambda_{6}-\lambda_{7}\right) e^{i \xi}\right]=-s_{2 \beta} \operatorname{Im}\left(\lambda_{5} e^{2 i \xi}\right), \\
c_{2 \beta} \operatorname{Re}\left[\left(\lambda_{6}+\lambda_{7}\right) e^{i \xi}\right]=\frac{1}{2} s_{2 \beta}\left(\lambda_{1}-\lambda_{2}\right), \\
\operatorname{Im}\left[\left(\lambda_{6}+\lambda_{7}\right) e^{i \xi}\right]=0 .
\end{gathered}
$$

Note that if the scalar potential parameters satisfy Eq. (9.7) then Eqs. (E1)-(E6) yield $c_{2 \beta}=\sin \xi=0$ as expected based on the corresponding scalar potential minimum conditions.

When written in a generic $\Phi$-basis, the form of the $\mathbb{Z}_{2}$ symmetry that is respected by the scalar potential and vacuum becomes somewhat obscure. Nevertheless, it is straightforward to check that if Eqs. (E1)-(E6) are satisfied, then the scalar potential and vacuum are invariant with respect to the following discrete symmetry of order 2 in the $\Phi$-basis,

$$
\begin{aligned}
\mathbb{Z}_{2 H}: \Phi_{1} & \rightarrow c_{2 \beta} \Phi_{1}+e^{-i \xi} s_{2 \beta} \Phi_{2}, \\
\Phi_{2} & \rightarrow e^{i \xi} s_{2 \beta} \Phi_{1}-c_{2 \beta} \Phi_{2},
\end{aligned}
$$

which can be obtained from Eq. (9.14) by setting $\theta=\frac{1}{2} \pi$ and applying a hypercharge $\mathrm{U}(1)_{\mathrm{Y}}$ transformation to remove an overall factor of $-i$. Note that the square of the $\mathbb{Z}_{2 H}$ transformation is equal to the identity, as advertised. As a final check, note that if we set $c_{2 \beta}=\sin \xi=0$ [cf. Eqs. (9.7) and (9.8)], then we can identify $\mathbb{Z}_{2 H}$ with $\Pi_{2}$. 
[1] G. Aad et al. (ATLAS Collaboration), Observation of a new particle in the search for the Standard Model Higgs boson with the ATLAS detector at the LHC, Phys. Lett. B 716, 1 (2012).

[2] S. Chatrchyan et al. (CMS Collaboration), Observation of a new boson at a mass of $125 \mathrm{GeV}$ with the CMS experiment at the LHC, Phys. Lett. B 716, 30 (2012).

[3] G. Aad et al. (ATLAS Collaboration), Combined measurements of Higgs boson production and decay using up to $80 \mathrm{fb}^{-1}$ of proton-proton collision data at $\sqrt{s}=13 \mathrm{TeV}$ collected with the ATLAS experiment, Phys. Rev. D 101, 012002 (2020).

[4] A. M. Sirunyan et al. (CMS Collaboration), Combined measurements of Higgs boson couplings in proton-proton collisions at $\sqrt{s}=13 \mathrm{TeV}$, Eur. Phys. J. C 79, 421 (2019).

[5] CMS Collaboration, Combined Higgs boson production and decay measurements with up to $137 \mathrm{fb}^{-1}$ of protonproton collision data at $\sqrt{s}=13 \mathrm{TeV}$, Report No. CMSPAS-HIG-19-005, 2020.

[6] ATLAS Collaboration, A combination of measurements of Higgs boson production and decay using up to $139 \mathrm{fb}^{-1}$ of proton-proton collision data at $\sqrt{s}=13 \mathrm{TeV}$ collected with the ATLAS experiment, Report No. ATLAS-CONF2020-027, 2020.

[7] D. A. Ross and M. J. G. Veltman, Neutral currents in neutrino experiments, Nucl. Phys. B95, 135 (1975).

[8] M. J. G. Veltman, Limit on mass differences in the Weinberg model, Nucl. Phys. B123, 89 (1977).

[9] M. S. Chanowitz, M. A. Furman, and I. Hinchliffe, Weak interactions of ultraheavy fermions, Phys. Lett. 78B, 285 (1978).

[10] D. Toussaint, Renormalization effects from superheavy Higgs particles, Phys. Rev. D 18, 1626 (1978).

[11] T. D. Lee, A theory of spontaneous T violation, Phys. Rev. D 8, 1226 (1973).

[12] J. F. Gunion, H. E. Haber, G. Kane, and S. Dawson, The Higgs Hunter's Guide (Westview Press, Boulder, CO, 2000).

[13] G. C. Branco, P. M. Ferreira, L. Lavoura, M. N. Rebelo, M. Sher, and J. P. Silva, Theory and phenomenology of twoHiggs-doublet models, Phys. Rep. 516, 1 (2012).

[14] S. L. Glashow and S. Weinberg, Natural conservation laws for neutral currents, Phys. Rev. D 15, 1958 (1977).

[15] E. A. Paschos, Diagonal neutral currents, Phys. Rev. D 15, 1966 (1977).

[16] A. W. El Kaffas, P. Osland, and O. M. Ogreid, Constraining the two-Higgs-doublet-model parameter space, Phys. Rev. D 76, 095001 (2007).

[17] A. Arbey, F. Mahmoudi, O. Stål, and T. Stefaniak, Status of the charged Higgs boson in two Higgs doublet models, Eur. Phys. J. C 78, 182 (2018).

[18] O. Eberhardt, A. P. Martínez, and A. Pich, Global fits in the aligned two-Higgs-doublet model, J. High Energy Phys. 05 (2021) 005.

[19] P. Fayet, Supergauge invariant extension of the Higgs mechanism and a model for the electron and its neutrino, Nucl. Phys. B90, 104 (1975).

[20] R. A. Flores and M. Sher, Higgs masses in the standard, multi-Higgs and supersymmetric models, Ann. Phys. (N.Y.) 148, 95 (1983).
[21] H. E. Haber and G. L. Kane, The search for supersymmetry: Probing physics beyond the Standard Model, Phys. Rep. 117, 75 (1985).

[22] J. F. Gunion and H. E. Haber, Higgs bosons in supersymmetric models (I), Nucl. Phys. B272, 1 (1986); Erratum, Nucl. Phys. 402, 567 (1993).

[23] L. Susskind, The gauge hierarchy problem, technicolor, supersymmetry, and all that, Phys. Rep. 104, 181 (1984).

[24] R. Barbieri, L. J. Hall, and V.S. Rychkov, Improved naturalness with a heavy Higgs: An alternative road to LHC physics, Phys. Rev. D 74, 015007 (2006).

[25] L. Lopez Honorez, E. Nezri, J. F. Oliver, and M. H. G. Tytgat, The inert doublet model: An archetype for dark matter, J. Cosmol. Astropart. Phys. 02 (2007) 028.

[26] E. Ma, Verifiable radiative seesaw mechanism of neutrino mass and dark matter, Phys. Rev. D 73, 077301 (2006).

[27] C. Arina, F. S. Ling, and M. H. G. Tytgat, IDM and iDM or the inert doublet model and inelastic dark matter, J. Cosmol. Astropart. Phys. 10 (2009) 018.

[28] A. Goudelis, B. Herrmann, and O. Stål, Dark matter in the inert doublet model after the discovery of a Higgs-like boson at the LHC, J. High Energy Phys. 09 (2013) 106.

[29] S. Davidson and H. E. Haber, Basis-independent methods for the two-Higgs-doublet model, Phys. Rev. D 72, 035004 (2005); Erratum, Phys. Rev. D 72, 099902 (2005).

[30] I. F. Ginzburg and M. Krawczyk, Symmetries of two Higgs doublet model and CP violation, Phys. Rev. D 72, 115013 (2005).

[31] A. Arhrib, E. Christova, H. Eberl, and E. Ginina, CP violation in charged Higgs production and decays in the complex two Higgs doublet model, J. High Energy Phys. 04 (2011) 089.

[32] A. Barroso, P. M. Ferreira, R. Santos, and J. P. Silva, Probing the scalar-pseudoscalar mixing in the $125 \mathrm{GeV}$ Higgs particle with current data, Phys. Rev. D 86, 015022 (2012).

[33] S. Inoue, M. J. Ramsey-Musolf, and Y. Zhang, $C P$-violating phenomenology of flavor conserving two Higgs doublet models, Phys. Rev. D 89, 115023 (2014).

[34] D. Fontes, J. C. Romão, and J. P. Silva, $h \rightarrow Z \gamma$ in the complex two Higgs doublet model, J. High Energy Phys. 12 (2014) 043.

[35] B. Grzadkowski, O. M. Ogreid, and P. Osland, Measuring $C P$ violation in two-Higgs-doublet models in light of the LHC Higgs data, J. High Energy Phys. 11 (2014) 084.

[36] D. Fontes, M. Mühlleitner, J. C. Romão, R. Santos, J. P. Silva, and J. Wittbrodt, The C2HDM revisited, J. High Energy Phys. 02 (2018) 073.

[37] R. Boto, T. V. Fernandes, H. E. Haber, J. C. Romão, and J. P. Silva, Basis-independent treatment of the complex 2HDM, Phys. Rev. D 101, 055023 (2020).

[38] I. Low, N. R. Shah, and X.-P. Wang, Higgs alignment and novel $C P$-violating observables in $2 \mathrm{HDM}$, arXiv: 2012.00773.

[39] P. S. B. Dev and A. Pilaftsis, Maximally symmetric two Higgs doublet model with natural Standard Model alignment, J. High Energy Phys. 12 (2014) 024; Erratum, J. High Energy Phys. 11 (2015) 147. 
[40] P. S. B. Dev and A. Pilaftsis, Natural alignment in the two Higgs doublet model, J. Phys. Conf. Ser. 873, 012008 (2017).

[41] P. M. Ferreira, H. E. Haber, and J. P. Silva, Generalized $C P$ symmetries and special regions of parameter space in the two-Higgs-doublet model, Phys. Rev. D 79, 116004 (2009).

[42] J.F. Gunion and H. E. Haber, The $C P$ conserving two Higgs doublet model: The approach to the decoupling limit, Phys. Rev. D 67, 075019 (2003).

[43] N. Craig and S. Thomas, Exclusive signals of an extended Higgs sector, J. High Energy Phys. 11 (2012) 083.

[44] N. Craig, J. Galloway, and S. Thomas, Searching for signs of the second Higgs doublet, arXiv:1305.2424.

[45] D. M. Asner et al., ILC Higgs white paper, arXiv: 1310.0763.

[46] M. Carena, I. Low, N. R. Shah, and C. E. M. Wagner, Impersonating the standard model Higgs boson: Alignment without decoupling, J. High Energy Phys. 04 (2014) 015.

[47] H. E. Haber, in Proceedings of the Toyama International Workshop on Higgs as a Probe of New Physics 2013 (HPNP2013), The Higgs Data and the Decoupling Limit, arXiv:1401.0152.

[48] P. Sikivie, L. Susskind, M. B. Voloshin, and V. I. Zakharov, Isospin breaking in technicolor models, Nucl. Phys. B173, 189 (1980).

[49] P. D. Mannheim, Effective low-energy custodial symmetry and weinberg mixing, Phys. Lett. 125B, 282 (1983).

[50] A. Pomarol and R. Vega, Constraints on $C P$ violation in the Higgs sector from the $\rho$ parameter, Nucl. Phys. B413, 3 (1994).

[51] J. M. Gerard and M. Herquet, A Twisted Custodial Symmetry in the Two-Higgs-Doublet Model, Phys. Rev. Lett. 98, 251802 (2007).

[52] B. Grzadkowski, M. Maniatis, and J. Wudka, The bilinear formalism and the custodial symmetry in the two-Higgsdoublet model, J. High Energy Phys. 11 (2011) 030.

[53] H. E. Haber and D. O'Neil, Basis-independent methods for the two-Higgs-doublet model III: The $C P$-conserving limit, custodial symmetry, and the oblique parameters $S$, $T, U$, Phys. Rev. D 83, 055017 (2011).

[54] M. Aiko and S. Kanemura, New scenario for aligned Higgs couplings originated from the twisted custodial symmetry at high energies, J. High Energy Phys. 02 (2021) 046.

[55] N. G. Deshpande and E. Ma, Pattern of symmetry breaking with two Higgs doublets, Phys. Rev. D 18, 2574 (1978).

[56] M. Maniatis, A. von Manteuffel, O. Nachtmann, and F. Nagel, Stability and symmetry breaking in the general twoHiggs-doublet model, Eur. Phys. J. C 48, 805 (2006).

[57] I. P. Ivanov, Minkowski space structure of the Higgs potential in 2HDM. II. Minima, symmetries, and topology, Phys. Rev. D 77, 015017 (2008).

[58] P. M. Ferreira, H. E. Haber, M. Maniatis, O. Nachtmann, and J. P. Silva, Geometric picture of generalized-CP and Higgs-family transformations in the two-Higgs-doublet model, Int. J. Mod. Phys. A 26, 769 (2011).

[59] R. A. Battye, G. D. Brawn, and A. Pilaftsis, Vacuum topology of the two Higgs doublet model, J. High Energy Phys. 08 (2011) 020.
[60] R. D. Peccei and H. R. Quinn, Constraints imposed by $C P$ conservation in the presence of instantons, Phys. Rev. D 16, 1791 (1977).

[61] H. E. Haber and Z. Surujon, A group-theoretic condition for spontaneous $C P$ violation, Phys. Rev. D 86, 075007 (2012).

[62] I. P. Ivanov, Two-Higgs-doublet model from the grouptheoretic perspective, Phys. Lett. B 632, 360 (2006).

[63] M. Abramowitz and I. A. Stegun, Handbook of Mathematical Functions (Dover Publications Inc., New York, 1972).

[64] R. Irvine, Beyond the Quadratic Formula (MAA Press, Providence, RI, 2013).

[65] J. F. Donoghue and L. F. Li, Properties of charged Higgs bosons, Phys. Rev. D 19, 945 (1979).

[66] H. Georgi and D. V. Nanopoulos, Suppression of flavor changing effects from neutral spinless meson exchange in gauge theories, Phys. Lett. 82B, 95 (1979).

[67] F. J. Botella and J. P. Silva, Jarlskog-like invariants for theories with scalars and fermions, Phys. Rev. D 51, 3870 (1995).

[68] G. C. Branco, L. Lavoura, and J. P. Silva, CP Violation (Oxford University Press, Oxford, UK, 1999).

[69] H. E. Haber and D. O'Neil, Basis-independent methods for the two-Higgs-doublet model II: The significance of $\tan \beta$, Phys. Rev. D 74, 015018 (2006); , Erratum, Phys. Rev. D 74, 059905 (2006).

[70] P. Draper, A. Ekstedt, and H. E. Haber, A natural mechanism for approximate Higgs alignment in the 2HDM, J. High Energy Phys. 05 (2021) 235. .

[71] A. Barroso, P. M. Ferreira, and R. Santos, Neutral minima in two-Higgs doublet models, Phys. Lett. B 652, 181 (2007).

[72] S. P. Martin, A supersymmetry primer, Adv. Ser. Dir. High Energy Phys. 18, 1 (1998).

[73] H. A. Weldon, The effects of multiple Higgs bosons on tree unitarity, Phys. Rev. D 30, 1547 (1984).

[74] I. F. Ginzburg and I. P. Ivanov, Tree-level unitarity constraints in the most general 2HDM, Phys. Rev. D 72, 115010 (2005).

[75] J. Horejsi and M. Kladiva, Tree-unitarity bounds for THDM Higgs masses revisited, Eur. Phys. J. C 46, 81 (2006).

[76] S. Kanemura and K. Yagyu, Unitarity bound in the most general two Higgs doublet model, Phys. Lett. B 751, 289 (2015).

[77] M. D. Goodsell and F. Staub, Improved unitarity constraints in two-Higgs-doublet-models, Phys. Lett. B 788, 206 (2019).

[78] P. M. Ferreira, H. E. Haber, and J. P. Silva, Basis invariant conditions for supersymmetry in the two-Higgs-doublet model, Phys. Rev. D 82, 016001 (2010).

[79] P. Draper and H. Rzehak, A review of Higgs mass calculations in supersymmetric models, Phys. Rep. 619, 1 (2016).

[80] H. E. Haber and R. Hempfling, Renormalization group improved Higgs sector of the minimal supersymmetric model, Phys. Rev. D 48, 4280 (1993).

[81] H. E. Haber and Y. Nir, Multiscalar models with a highenergy scale, Nucl. Phys. B335, 363 (1990). 
[82] J. Bernon, J. F. Gunion, H. E. Haber, Y. Jiang, and S. Kraml, Scrutinizing the alignment limit in two-Higgsdoublet models, Part 1: $m_{h}=125 \mathrm{GeV}$, Phys. Rev. D 92, 075004 (2015).

[83] H. E. Haber and O. Stål, New LHC benchmarks for the $C P$-conserving two-Higgs-doublet model, Eur. Phys. J. C 75, 491 (2015); , Erratum, Eur. Phys. J. C 76, 312 (2016).

[84] J. Bernon, J. F. Gunion, H. E. Haber, Y. Jiang, and S. Kraml, Scrutinizing the alignment limit in two-Higgsdoublet models, Part 2: $m_{H}=125 \mathrm{GeV}$, Phys. Rev. D 93, 035027 (2016).

[85] N. Darvishi and A. Pilaftsis, Natural alignment in multiHiggs doublet models, Proc. Sci., CORFU2019 (2020) 064 [arXiv:2004.04505].

[86] G. 't Hooft, in Recent Developments in Gauge Theories, edited by G. 't Hooft et al. NATO Advanced Study Institute series: Series B, Physics Vol. 59, (Plenum Press, New York, London, 1980), pp. 135-157.

[87] A. Pilaftsis, On the classification of accidental symmetries of the two Higgs doublet model potential, Phys. Lett. B 706, 465 (2012).

[88] N. Darvishi and A. Pilaftsis, Classifying accidental symmetries in multi-Higgs doublet models, Phys. Rev. D 101, 095008 (2020).

[89] P. M. Ferreira and J. P. Silva, A two-Higgs doublet model with remarkable $C P$ properties, Eur. Phys. J. C 69, 45 (2010).

[90] N. Darvishi and A. Pilaftsis, Quartic coupling unification in the maximally symmetric 2HDM, Phys. Rev. D 99, 115014 (2019).

[91] P. Draper, H. E. Haber, and J. T. Ruderman, Partially natural two Higgs doublet models, J. High Energy Phys. 06 (2016) 124.

[92] I. Antoniadis, K. Benakli, A. Delgado, and M. Quiros, A new gauge mediation theory, Adv. Stud. Theor. Phys. 2, 645 (2008), http://www.m-hikari.com/astp/astp2008/ astp13-16-2008/delgadoASTP13-16-2008.pdf.

[93] J. Ellis, J. Quevillon, and V. Sanz, Doubling up on supersymmetry in the Higgs sector, J. High Energy Phys. 10 (2016) 086.

[94] K. Benakli, M. D. Goodsell, and S. L. Williamson, Higgs alignment from extended supersymmetry, Eur. Phys. J. C 78, 658 (2018).

[95] K. Benakli, Y. Chen, and G. Lafforgue-Marmet, R-symmetry for Higgs alignment without decoupling, Eur. Phys. J. C 79, 172 (2019).

[96] K. Lane and W. Shepherd, Natural stabilization of the Higgs boson's mass and alignment, Phys. Rev. D 99, 055015 (2019).
[97] E. Eichten and K. Lane, Higgs alignment and the top quark, arXiv:2102.07242.

[98] G. C. Branco, Spontaneous CP nonconservation and natural flavor conservation: A minimal model, Phys. Rev. D 22, 2901 (1980).

[99] G. C. Branco and M. N. Rebelo, The Higgs mass in a model with two scalar doublets and spontaneous $C P$ violation, Phys. Lett. 160B, 117 (1985).

[100] C. Lin, C. Lee, and Y.-W. Yang, Spontaneous CP violation in the extended standard models, Chin. J. Phys. 32, 41 (1994), https://www.ps-taiwan.org/cjp/download.php? type $=$ paper $\&$ vol $=32 \&$ num $=1 \&$ page $=41$.

[101] G. T. Gilbert, Positive definite matrices and Sylvester's criterion, Am. Math. Mon. 98, 44 (1991).

[102] J. F. Gunion and H. E. Haber, Conditions for $C P$ violation in the general two-Higgs-doublet model, Phys. Rev. D 72, 095002 (2005).

[103] See, e.g., S. R. Garcia and R. A. Horn, A Second Course in Linear Algebra (Cambridge University Press, Cambridge, England, 2017).

[104] G. Ecker, W. Grimus, and H. Neufeld, A standard form for generalized CP transformations, J. Phys. A 20, L807 (1987).

[105] G. Ecker, W. Grimus, and W. Konetschny, Quark mass matrices in left-right symmetric gauge theories, Nucl. Phys. B191, 465 (1981).

[106] G. Ecker, W. Grimus, and H. Neufeld, Spontaneous $C P$ violation in left-right symmetric gauge theories, Nucl. Phys. B247, 70 (1984).

[107] H. Neufeld, W. Grimus, and G. Ecker, Generalized $C P$ invariance, neutral flavor conservation and the structure of the mixing matrix, Int. J. Mod. Phys. A 03, 603 (1988).

[108] G. C. Branco, M. N. Rebelo, and J. I. Silva-Marcos, $C P$-odd invariants in models with several Higgs doublets, Phys. Lett. B 614, 187 (2005).

[109] M. Maniatis, A. von Manteuffel, and O. Nachtmann, $C P$ violation in the general two-Higgs-doublet model: A geometric view, Eur. Phys. J. C 57, 719 (2008).

[110] M. Maniatis, A. von Manteuffel, and O. Nachtmann, A new type of $C P$ symmetry, family replication and fermion mass hierarchies, Eur. Phys. J. C 57, 739 (2008).

[111] M. Maniatis and O. Nachtmann, On the phenomenology of a two-Higgs-doublet model with maximal $C P$ symmetry at the LHC, J. High Energy Phys. 05 (2009) 028.

[112] M. Maniatis and O. Nachtmann, On the phenomenology of a two-Higgs-doublet model with maximal $C P$ symmetry at the LHC. II. Radiative effects, J. High Energy Phys. 04 (2010) 027. 